\title{
Managing concerns about falls : fear of falling and avoidance of activity in older people
}

Citation for published version (APA):

Zijlstra, G. A. R. (2007). Managing concerns about falls : fear of falling and avoidance of activity in older people. [Doctoral Thesis, Maastricht University]. Maastricht University.

https://doi.org/10.26481/dis.20080228gz

Document status and date:

Published: 01/01/2007

DOI:

$10.26481 /$ dis.20080228gz

Document Version:

Publisher's PDF, also known as Version of record

\section{Please check the document version of this publication:}

- A submitted manuscript is the version of the article upon submission and before peer-review. There can be important differences between the submitted version and the official published version of record.

People interested in the research are advised to contact the author for the final version of the publication, or visit the DOI to the publisher's website.

- The final author version and the galley proof are versions of the publication after peer review.

- The final published version features the final layout of the paper including the volume, issue and page numbers.

Link to publication

\footnotetext{
General rights rights.

- You may freely distribute the URL identifying the publication in the public portal. please follow below link for the End User Agreement:

www.umlib.nl/taverne-license

Take down policy

If you believe that this document breaches copyright please contact us at:

repository@maastrichtuniversity.nl

providing details and we will investigate your claim.
}

Copyright and moral rights for the publications made accessible in the public portal are retained by the authors and/or other copyright owners and it is a condition of accessing publications that users recognise and abide by the legal requirements associated with these

- Users may download and print one copy of any publication from the public portal for the purpose of private study or research.

- You may not further distribute the material or use it for any profit-making activity or commercial gain

If the publication is distributed under the terms of Article $25 \mathrm{fa}$ of the Dutch Copyright Act, indicated by the "Taverne" license above, 


\section{Managing concerns about falls}

Fear of falling and avoidance of activity in older people 
The studies presented in this thesis were performed at the School for Public Health and Primary Care (Caphri) of the Maastricht University. Caphri participates in the Netherlands School of Primary Care Research (CaRe), re-acknowledged by the Royal Netherlands Academy of Arts and Sciences (KNAW) in 2006.

ISBN: 978-90-8590-028-3

ISBN: 978-90-8590-029-0 (audio visuele media)

Cover art:

'Balancing Act' by Banx, www.banx.com.au

Design and lay-out:

D\&L Graphics, www.dlgraphics.nl

Printed by:

Schrijen Lippertz Huntjens, Sittard

(C) 2007, G.A.R. Zijlstra, Maastricht

All rights reserved. No part of this thesis may be reproduced, stored in a retrieval system, or transmitted, in any form or by any means, electronic, mechanical, photocopying, recording, or otherwise, without written permission from the author or from the publisher holding the copyright of the published article. 


\title{
Managing concerns about falls
}

Fear of falling and avoidance of activity in older people

\author{
PROEFSCHRIFT \\ ter verkrijging van de graad van doctor \\ aan de Universiteit Maastricht, \\ op gezag van de Rector Magnificus, \\ Prof. mr. G.P.M.F. Mols, \\ volgens het besluit van het College van Decanen, \\ in het openbaar te verdedigen \\ op donderdag 28 februari 2008 om 16.00 uur
}

door

Gertrud Akke Rixt Zijlstra 


\section{Promotores}

Prof. dr. G.I.J.M. Kempen

Prof. dr. J.Th.M. van Eijk

\section{Copromotor}

Dr. J.C.M. van Haastregt

\section{Beoordelingscommissie}

Prof. dr. J.A. Knottnerus (voorzitter)

Prof. dr. P.T.A.M. Lips (VUmc)

Prof. dr. F.R.J. Verhey

Prof. dr. J.W.S. Vlaeyen

Prof. dr. N.K. de Vries

ZonMw - The Netherlands Organisation for Health Research and Development (grant 014-91-052), the School for Public Health and Primary Care (Caphri), and the Faculty of Health, Medicine and Life Sciences of the Maastricht University are gratefully acknowledged for funding the studies in this thesis. 
Fear is inevitable, and it is not always bad. Harness your fear and use it to your advantage.

John Grisham, Bleachers 2003 
(20)

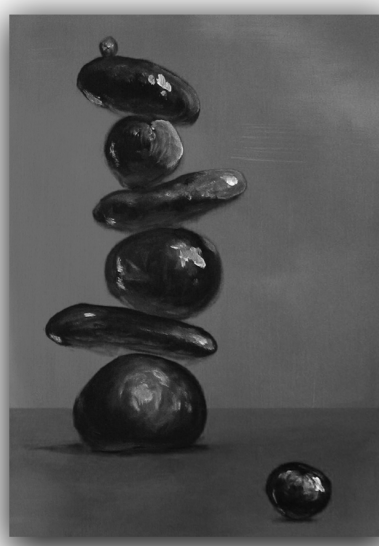




\section{Contents}

Chapter 1 General introduction 9

Chapter 2 Prevalence and correlates of fear of falling and associated avoidance of activity in the general population of community-living older people

Chapter 3 Interventions to reduce fear of falling in community-living older people: a systematic review

Chapter 4 Reducing fear of falling and avoidance of activity in elderly persons: the development of a Dutch version of an American intervention

Chapter 5 Evaluating an intervention to reduce fear of falling and associated activity restriction in elderly persons: design of a randomised controlled trial

Chapter 6 Feasibility of a cognitive behavioural group intervention to reduce fear of falling and associated avoidance of activity in community-living older people: a process evaluation

Chapter 7 Effectiveness of a cognitive behavioural group intervention on fear of falling and associated avoidance of activity in community-living older people: a randomised controlled trial

Chapter 8 Mediating effects of psychosocial factors on fear of falling and daily activity in a cognitive behavioural intervention

Chapter 9 General discussion

Chapter 10 Summary

Samenvatting

Co-authors and affiliations

Dankwoord / Acknowledgement

Publications

About the author 



\section{CHAPTER 1}

General introduction 


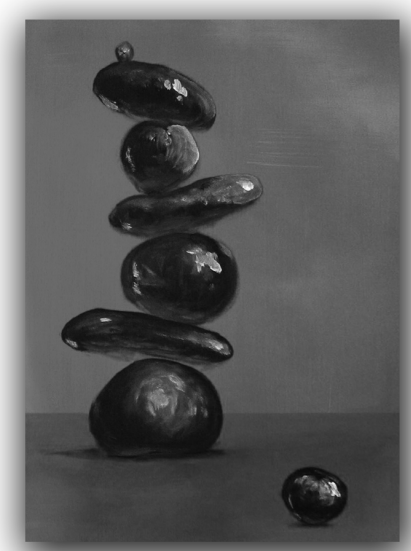




\section{Challenges in an aging society}

Increased aging of the society is common in many countries. In The Netherlands, the proportion of people aged 65 years and over increased from 7.7 to $14.3 \%$ between 1950 and 2006, and is expected to rise to $23.6 \%$ in $2050 .{ }^{1}$ In addition, the life expectancy at birth increased from 70.3 to 77.6 years for men and from 72.6 to 81.7 years for women between 1950 and 2006. A subsequent gradual increase to 81.5 and 84.2 years for men and women, respectively, is estimated until the year 2050. ${ }^{1}$ Yet, life expectancy is no indicator for the years one lives in good health; these years are indicated by the healthy life expectancy. Fortunately, an increase in healthy life expectancy, in which health is reflected by self-rated general health, is shown over time. ${ }^{2}$ This might be explained by effective health care and prevention strategies, including those that facilitate self-management of diseases and functional limitations in order to prevent disability and to enhance independent living and quality of life. Despite these encouraging findings regarding increased healthy life expectancy, a substantial increase of most physical and mental diseases, and functional limitations is estimated due to the increased aging of the society and the commonness of morbidity and co-morbidity in older people. ${ }^{3}$ This implies that during the next decades our aging society will increasingly be challenged by health concerns prevalent in older populations.

Falls are a major health concern in older populations and require effective prevention. ${ }^{5-9}$ In western societies, approximately one-third of the community-living people aged 65 years or older falls at least once a year; ${ }^{10-13}$ this number increases with age. ${ }^{14-17}$ Although most falls do not result in serious injuries, ${ }^{17-19}$ falls are a major cause of worldwide disability-adjusted life years, which indicates burdened and lost life years due to disability and premature mortality. ${ }^{20}$ Common fall-related consequences include injuries, ${ }^{18} 21-23$ decreased physical functioning or activities of daily life, ${ }^{23-26}$ loss of independence, ${ }^{27}$ increased health care utilisation ${ }^{23} 28$ and mortality. ${ }^{21}$ Besides these, falls can have other consequences as well, such as fear of falling and avoidance of activities due to fear of falling, which are frequently reported psychosocial and behavioural consequences of falls. ${ }^{102529}$ Given these consequences and their associated costs, numerous strategies aimed at preventing falls have been evaluated as illustrated in several reviews of the literature. 679

Although fear of falling was initially solely considered a consequence of a recent fall, along with associated avoidance of activity, this fear is currently considered a health concern that deserves distinct attention. ${ }^{30}$ In general, about $50 \%$ of the community-living older people report fear of falling $102529-43$ and this fear showed to be prevalent in non-fallers as well. ${ }^{29} 3044-46$ This implies that, compared to falls, fear of falling is a more widespread concern. ${ }^{44}$ In addition, about $40 \%$ of the communityliving older people report avoidance of activities due to this fear of falling. ${ }^{10293641}$ These prevalence rates and the adverse consequences, like decreased physical, psychological and social functioning, 324547 associated with fear of falling and avoidance behaviour justify distinctive prevention strategies aimed at managing these concerns about falling. In the international literature, however, very little attention has been paid to the management of fear of falling and associated avoidance of activity 
as specific challenges in an aging society. For that reason, the main subject of this thesis is managing fear of falling and associated avoidance of activity in communityliving older people.

\section{Definitions}

Fall-related psychological and behavioural consequences have initially been identified as an intense fear or a fear causing specific phobic behaviour in older people who received psychiatric care, ${ }^{48}$ or medical or rehabilitative care after a fall. ${ }^{49} 50$ Despite the absence of physical causes, these patients showed a hesitancy and irregularity in mobility and were inclined to seek support in nearby objects. ${ }^{49}$ Medical-oriented concepts such as post-fall syndrome ${ }^{49}$ and ptophobia ${ }^{50}$ were then applied to describe this fear. During the past two decades more attention has been paid to fall-related psychological aspects in older people. Currently, the concept of fear of falling is commonly applied; yet, other psychological concepts related to falls or performing safe behaviour are applied as well, including worry about falling, concern about falling, or self-efficacy or confidence to perform activities without falling or losing balance. ${ }^{51-53}$ This is reflected in a systematic review on measures that assess psychological outcomes related to falls. ${ }^{54}$

Tinetti and Powell defined fear of falling as "a lasting concern about falling that leads to an individual avoiding activities that he/she remains capable of performing" ${ }^{5}$. In this thesis we elaborated on this definition and, unless stated otherwise, fear of falling or being afraid of falling refers to a concern about falling; all three terms are considered equivalent. Associated avoidance of activity refers to avoiding activities, which one is still capable of performing, due to this concern of falling. A fall is defined as an event that results in a person coming to rest inadvertently on the ground or lower level. ${ }^{56}$ This thesis is aimed at community-living older people. Unless stated otherwise, community-living older people are defined as people 70 years of age or older who live independently in the community. People who independently live in sheltered accommodations are considered community-living; people living in care facilities, such as nursing homes, are not considered community-living.

\section{Fear of falling and associated avoidance of activity}

Fear of falling and associated avoidance of activity are common in older people. Despite observed hesitance to discuss fear of falling, 3357 about $50 \%$ of the communityliving older people report fear of falling (range 20 to $85 \%$ ) $102529-43$ and about $40 \%$ report avoidance of activities due to fear of falling (range 15 to 55\%). ${ }^{10} 293641$ Community-living older people not only ranked the fear of falling and related injury highest compared to other fears, such as the fear to experience serious health problems or financial distress, but also ranked the odds of such a fall occurring and the frequency of thinking of this event highest. ${ }^{29}$ This underscores the commonness of fear of falling. Yet, little is known about the development of fear of falling. Given that fear of falling is not solely reported by fallers, factors associated with aging, such 
as frailty and impaired vision, general anxiety or knowing someone who has sustained a serious fall, might be initiators of fear of falling as well. This fear might also be dictated by people's perceptions, for instance on falls. Research showed that older people perceive falls as a sign of weakness and frailty preceding loss of independence ${ }^{38}$ ${ }^{58}$ or experience it as psychosocially damaging, for example by feelings of foolishness. ${ }^{38}$

With regard to fear of falling, falls and quality of life, older people tend to exercise precaution on the one hand and strive for independence in daily life on the other. ${ }^{59}$ Throughout this continuous balancing act, maintaining appropriate levels of fear of falling with respect to the performance of particular physical activities is essential because inappropriate levels of fear may lead to self-imposed restrictions of activity. Two decades ago Vellas and colleagues suggested that fear of falling might aggravate and accelerate the process of aging by initiating or contributing to a debilitating spiral of loss of confidence and reduced activity, which may lead to loss of independence. ${ }^{60}$ Findings of subsequent studies have strengthened this suggestion. Fear of falling or related concepts showed to be associated with a variety of adverse factors, including depressive symptoms, ${ }^{32} 3547$ general anxiety, ${ }^{34} 4761$ neuroticism, ${ }^{62}$ decreased satisfaction

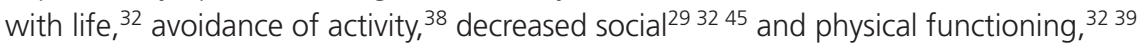
456163 poor health, 3062 increased frailty, 253235444664 falls, 13323336394043626566 decreased ability to manage falls, ${ }^{61}$ reduced quality of life 30323967 and increased institutionalisation. ${ }^{30}$ Although less extensively studied, similar factors have shown to be associated with avoidance of activity due to fear of falling: depressive symptoms, ${ }^{37}$ decreased physical functioning, ${ }^{376869}$ increased frailty ${ }^{65} 68$ and falls. ${ }^{37} 65$ Research also showed that, if untreated, fear of falling is likely a persistent concern in older people. ${ }^{36}$ 70 Obviously, the adverse consequences of fear of falling and associated avoidance of activity will affect public expenditure as health care utilisation increases.

The aforementioned indicates that, generally, fear of falling is not a fear, which during the course of time diminishes or is easily managed by older people themselves. In contrast, older people may be reluctant to seek support as they are hesitant to discuss fear of falling ${ }^{33} 57$ or accept fear of falling as part of the process of aging. ${ }^{71}$ Overall, these findings are further warranty for the development of strategies to prevent inappropriate levels of fear of falling and unnecessary avoidance of activity in community-living older people.

\section{Managing fear of falling and avoidance of activity}

Due to the broad variety of factors associated with fear of falling and associated avoidance of activity, the approach to manage this fear and avoidance by addressing multiple factors, i.e. physical, psychological and social factors, is widely acknowledged. ${ }^{35}$ 4561 72-74 However, only a few prevention strategies aimed at managing fear of falling and avoidance of activity in community-living older people have been developed and thoroughly evaluated. In 1998 two research groups reported on different interventions aimed at reducing fear of falling and increasing physical activity. Brouwer and colleagues evaluated an education and an activity programme of 8 weekly sessions of 1 hour and showed statistically significant reductions in fear of falling, as well as a 
tendency of increased physical activity for both programmes. ${ }^{73} \mathrm{~A}$ methodological limitation of this study is the lack of a control group, which is imperative to control for unknown or unmeasured confounders. A randomised controlled trial has been performed by Tennstedt and colleagues in Boston who evaluated a cognitive behavioural intervention called 'A Matter of Balance'. ${ }^{75}$ During this group intervention of eight sessions held twice a week multiple factors were addressed, for instance beliefs on falls, physical activity and safe behaviour. Compliers with this intervention showed short-term and long-term statistically significant improvements in falls selfefficacy, a concept related to fear of falling, and mobility.

More recently, while the studies presented in this thesis were in progress, the results of two randomised controlled trials evaluating interventions aimed at reducing fear of falling have been published. First, in 2004 Clemson and colleagues reported statistically significant effects for the intervention group on fear of falling after participation in a multifactorial group intervention. ${ }^{76}$ This intervention comprised eight sessions of 2 hours in which home and environment safety, medication management, vision screening and physical exercises were addressed. Yet, no favourable effects were shown regarding increased activity. Second, in 2006 Zhang and colleagues published results of an intense intervention of 8 weeks with daily sessions of 1 hour during which simplified tai chi exercises were performed. ${ }^{77}$ The intervention group improved significantly regarding fear of falling, balance and flexibility. Data on daily activities were not reported.

Findings of these aforementioned trials, which evaluated appropriate interventions, further underscore the importance of managing fear of falling and avoidance of activity by including multiple factors in the intervention. Although imperative for developing strategies to successfully manage fear of falling and associated avoidance of activity, information on modifiable factors that might be important contributors to such management is limited. The research group in Boston has identified factors in prior studies, which were subsequently incorporated in the intervention protocol of the cognitive behavioural intervention aimed at reducing fear of falling and increasing activity. These factors included talking about falls, maladaptive ideas regarding fear of falling, falls and physical activity, awareness of the problem and possible adaptation strategies, perceived control over falling, perceived ability to manage falls, assertiveness, environmental safety, risk-taking behaviour, social activity and physical fitness. ${ }^{29} 336171$ Due to the favourable outcomes of their randomised controlled trial ${ }^{75}$ and their well-founded intervention by means of cognitive behavioural strategies, ${ }^{78}$ application of this intervention elsewhere seems promising.

\section{Studies in The Netherlands}

Not until recently fear of falling and associated avoidance of activity in community-living older people received little attention in Dutch research. In varying extent of interest for fear of falling, research papers have addressed related aspects, including the prevalence of fear of falling, ${ }^{42} 4379-81$ the association, or lack of association, with other factors, such as compliance to hip protector use, ${ }^{132343667982}$ the assessment of fear of falling or a related psychological concept, ${ }^{83-85}$ and its reduction after participation in a 5-week 
exercise programme. ${ }^{86}$ In by far the most of these studies avoidance of activity due to fear of falling has not been addressed. Moreover, in The Netherlands a prevention strategy to help older people to manage this fear and avoidance behaviour is lacking.

\section{Objectives of this thesis}

The main objective of this thesis is to evaluate a cognitive behavioural intervention, based on the intervention developed by Tennstedt and colleagues, aimed at reducing fear of falling and associated avoidance of activity in community-living older people in the Dutch context. ${ }^{75}$ However, little is known about fear of falling and associated avoidance of activity in Dutch older people and potential relevant interventions for reducing fear of falling. Hence, the three objectives of this thesis are:

1. to study the prevalence and correlates of fear of falling and associated avoidance of activity in community-living older people in The Netherlands;

2. to obtain insight into the international literature on interventions that reduce fear of falling in community-living older people;

3. to evaluate a cognitive behavioural intervention aimed at reducing fear of falling and associated avoidance of activity in community-living older people in The Netherlands.

Findings may lead to awareness regarding fear of falling and associated avoidance of activity in Dutch older people and improved recognition of these concerns about falls in this population. In addition, the findings will contribute to a better understanding of interventions that reduce fear of falling and, in particular, of a cognitive behavioural intervention aimed at reducing fear of falling and associated avoidance of activity in community-living older people in The Netherlands.

\section{Outline of this thesis}

The objectives of this thesis are addressed in the different chapters of this thesis. In Chapter 2 and 3 the first and second objective, respectively, are addressed. The subsequent part of this thesis, i.e. Chapters 4 till 8, reports on the evaluation of the cognitive behavioural intervention in Dutch community-living older people.

In Chapter 2 the prevalence of fear of falling and associated avoidance of activity in community-living older people in The Netherlands is described. Additionally, associations between several socio-demographic and health-related variables and fear of falling and associated avoidance of activity are presented. Data of this crosssectional study among 4,031 participants was collected during the procedure in which Dutch community-living older people were selected for the trial evaluating the cognitive behavioural intervention.

Chapter 3 presents the findings of a systematic review on interventions to reduce fear of falling in community-living older people. In addition to the effects of the interventions on fear of falling, data on the methodological quality of the 19 included randomised controlled trials and data on the process characteristics regarding the interventions are presented. 
Chapter 4 provides insight into the development process of a Dutch version of an American cognitive behavioural intervention aimed at reducing fear of falling and associated avoidance of activity in community-living older people. Activities undertaken during the development process to facilitate successful implementation of this intervention in a new setting are presented in detail.

The design of the randomised controlled trial that evaluated the aforementioned intervention is described in Chapter 5. Information is provided on the inclusion of participants, the procedure of randomisation, the intervention and the data collection.

In Chapter 6 the outcomes of the process evaluation that was performed alongside the randomised controlled trial are described. Data was collected from 168 participants and 6 facilitators of the intervention and addressed the performance according to protocol, participants' attendance, participants' adherence, and the opinion of the participants and facilitators regarding the intervention.

Chapter 7 reports on the effects of the Dutch cognitive behavioural intervention on fear of falling, avoidance of activity, perceived control over falling, daily activity and several other outcomes. Intervention effects directly after the intervention, and at 6 and 12 months after the intervention are presented.

In Chapter 8 the mediating effects of eight psychosocial factors on trajectories of fear of falling and daily activity in the cognitive behavioural intervention are reported. The potential mediators comprised control beliefs, self-efficacy beliefs, outcome expectations and social interactions. Data of the mediators and the outcome measures were assessed before the intervention, directly after the intervention, and at 6 and 12 months after the intervention and as such incorporated in the analyses.

Chapter 9, the final chapter of this thesis, presents a general discussion with respect to the main findings, and strengths and limitations of the presented studies. Implications for practice and future research are provided. 


\section{References}

1. CBS. Statline database: population. Retrieved 24 January 2007, from http:// statline.cbs.nl, 2007.

2. van den Berg Jeths A, Timmermans JM, Hoeymans N, Woittiez IB. Ouderen nu en in de toekomst. Gezondheid, verpleging en verzorging 2000-2020. Bilthoven/Den Haag: RIVM/SCP, 2004.

3. Volksgezondheid Toekomst Verkenning NKV, versie 3.9. Bilthoven: RIVM, http://www. nationaalkompas.nl, 2007.

4. Gezondheidsraad. Vergrijzen met ambitie. Den Haag: Gezondheidsraad, 2005.

5. Guideline for the prevention of falls in older persons. American Geriatrics Society, British Geriatrics Society, and American Academy of Orthopaedic Surgeons Panel on Falls Prevention. J Am Geriatr Soc 2001;49(5):664-72.

6. Gillespie LD, Gillespie WJ, Robertson MC, et al. Interventions for preventing falls in elderly people. Cochrane Database Syst Rev 2003(4):CD000340.

7. Chang JT, Morton SC, Rubenstein LZ, et al. Interventions for the prevention of falls in older adults: systematic review and meta-analysis of randomised clinical trials. BMJ 2004;328(7441):680.

8. Todd C, Skelton D. What are the main risk factors for falls among older people and what are the most effective interventions to prevent these falls? Copenhagen: WHO Regional Office for Europe (Health Evidence Network report). Retrieved 26 March 2006 from http://www.euro.who.int/document/ E82552.pdf, 2004.

9. Kannus $P$, Sievanen $H$, Palvanen $M$, et al. J. Prevention of falls and consequent injuries in elderly people. Lancet 2005;366(9500):1885-93.

10. Tinetti ME, Speechley M, Ginter SF. Risk factors for falls among elderly persons living in the community. N Engl J Med 1988;319(26):1701-7.

11. Campbell AJ, Borrie MJ, Spears GF. Risk factors for falls in a community-based prospective study of people 70 years and older. J Gerontol 1989;44(4):M112-7.

12. Nevitt MC, Cummings SR, Kidd S, et al. Risk factors for recurrent nonsyncopal falls. A prospective study. JAMA 1989;261(18):2663-8.

13. Tromp AM, Pluijm SM, Smit JH, et al. Fall-risk screening test: a prospective study on predictors for falls in community-dwelling elderly. J Clin Epidemio/ 2001;54(8):837-44.

14. Prudham D, Evans JG. Factors associated with falls in the elderly: a community study. Age Ageing 1981;10(3):141-6.

15. Blake AJ, Morgan K, Bendall MJ, et al. Falls by elderly people at home: prevalence and associated factors. Age Ageing 1988;17(6):365-72.

16. O'Loughlin JL, Robitaille $Y$, Boivin JF, et al. Incidence of and risk factors for falls and injurious falls among the community-dwelling elderly. Am J Epidemio/ 1993;137(3):34254.

17. van Weel $C$, Vermeulen $H$, van den Bosch W. Falls, a community care perspective. Lancet 1995;345(8964):1549-51.

18. Nevitt MC, Cummings SR, Hudes ES. Risk factors for injurious falls: a prospective study. J Gerontol 1991;46(5):M164-70.

19. Reinsch S, MacRae P, Lachenbruch PA, et al. Attempts to prevent falls and injury: a prospective community study. Gerontologist 1992;32(4):450-6. 
20. Murray CJ, Lopez AD. Global mortality, disability, and the contribution of risk factors: Global Burden of Disease Study. Lancet 1997;349(9063):1436-42.

21. Kannus P, Parkkari J, Koskinen S, et al. Fall-induced injuries and deaths among older adults. JAMA 1999;281(20):1895-9.

22. Kannus $P$, Palvanen M, Niemi S, et al. Increasing number and incidence of fall-induced severe head injuries in older adults: nationwide statistics in Finland in 1970-1995 and prediction for the future. Am J Epidemio/ 1999;149(2):143-50.

23. Stel VS, Smit JH, Pluijm SM, et al. Consequences of falling in older men and women and risk factors for health service use and functional decline. Age Ageing 2004;33(1):5865.

24. Kiel DP, O'Sullivan P, Teno JM, et al. Health care utilization and functional status in the aged following a fall. Med Care 1991;29(3):221-8.

25. Vellas BJ, Wayne SJ, Romero LJ, et al. Fear of falling and restriction of mobility in elderly fallers. Age Ageing 1997;26(3):189-93.

26. Chu LW, Chiu AY, Chi I. Impact of falls on the balance, gait, and activities of daily living functioning in community-dwelling chinese older adults. J Gerontol A Biol Sci Med Sci 2006;61(4):399-404.

27. Tinetti ME, Williams CS. Falls, injuries due to falls, and the risk of admission to a nursing home. N Engl J Med 1997;337(18):1279-84.

28. Alexander BH, Rivara FP, Wolf ME. The cost and frequency of hospitalization for fallrelated injuries in older adults. Am J Public Health 1992;82(7):1020-3.

29. Howland J, Peterson EW, Levin WC, et al. Fear of falling among the communitydwelling elderly. J Aging Health 1993;5(2):229-43.

30. Cumming RG, Salkeld G, Thomas M, et al. Prospective study of the impact of fear of falling on activities of daily living, SF-36 scores, and nursing home admission. J Gerontol A Biol Sci Med Sci 2000;55(5):M299-305.

31. Underwood MR, Parsons S, Eldridge SM, et al. Asking older people about fear of falling did not have a negative effect. J Clin Epidemio/ 2006;59(6):629-34.

32. Arfken CL, Lach HW, Birge SJ, et al. The prevalence and correlates of fear of falling in elderly persons living in the community. Am J Public Health 1994; 84(4):565-70.

33. Howland J, Lachman ME, Peterson EW, et al. Covariates of fear of falling and associated activity curtailment. Gerontologist 1998;38(5):549-55.

34. Drozdick LW, Edelstein BA. Correlates of fear of falling in older adults who have experienced a fall. J Clin Geropsychol 2001;7(1):1-13.

35. Kressig RW, Wolf SL, Sattin RW, et al. Associations of demographic, functional, and behavioral characteristics with activity-related fear of falling among older adults transitioning to frailty. J Am Geriatr Soc 2001;49(11):1456-62.

36. Friedman SM, Munoz B, West SK, et al. Falls and fear of falling: which comes first? A longitudinal prediction model suggests strategies for primary and secondary prevention. J Am Geriatr Soc 2002;50(8):1329-35.

37. Murphy SL, Williams CS, Gill TM. Characteristics associated with fear of falling and activity restriction in community-living older persons. J Am Geriatr Soc 2002;50(3):516-20.

38. Yardley L, Smith H. A prospective study of the relationship between feared consequences of falling and avoidance of activity in community-living older people. Gerontologist 2002;42(1):17-23. 
39. Li F, Fisher KJ, Harmer P, et al. Fear of falling in elderly persons: association with falls, functional ability, and quality of life. J Gerontol B Psychol Sci Soc Sci 2003;58(5):P28390.

40. Fletcher PC, Hirdes JP. Restriction in activity associated with fear of falling among community-based seniors using home care services. Age Ageing 2004;33(3):273-9.

41. Wilson MM, Miller DK, Andresen EM, et al. Fear of falling and related activity restriction among middle-aged African Americans. J Gerontol A Biol Sci Med Sci 2005;60(3):35560.

42. Stalenhoef PA, Diederiks JP, Knottnerus JA, et al. The construction of a patient recordbased risk model for recurrent falls among elderly people living in the community. Fam Pract 2000;17(6):490-6.

43. Pluijm SMF. Predictors and consequences of falls and fractures in the elderly. Vrije Universiteit Amsterdam, 2001.

44. Maki BE, Holliday PJ, Topper AK. Fear of falling and postural performance in the elderly. J Gerontol 1991;46(4):M123-31.

45. Tinetti ME, Mendes de Leon CF, Doucette JT, et al. Fear of falling and fall-related efficacy in relationship to functioning among community-living elders. J Geronto/ 1994; 49(3):M140-7.

46. Myers AM, Powell LE, Maki BE, et al. Psychological indicators of balance confidence: relationship to actual and perceived abilities. J Gerontol A Biol Sci Med Sci 1996; 51(1):M37-43.

47. Gagnon N, Flint AJ, Naglie G, et al. Affective correlates of fear of falling in elderly persons. Am J Geriatr Psychiatry 2005;13(1):7-14.

48. Marks I, Bebbington P. Space phobia: syndrome or agoraphobic variant? Br Med J 1976;2(6031):345-7.

49. Murphy J, Isaacs B. The post-fall syndrome. A study of 36 elderly patients. Gerontology 1982;28(4):265-70

50. Bhala RP, O'Donnell J, Thoppil E. Ptophobia. Phobic fear of falling and its clinical management. Phys Ther 1982;62(2):187-90.

51. Tinetti ME, Richman D, Powell L. Falls efficacy as a measure of fear of falling. J Gerontol 1990;45(6):P239-43.

52. Powell LE, Myers AM. The Activities-specific Balance Confidence (ABC) Scale. J Gerontol A Biol Sci Med Sci 1995;50a(1):M28-34.

53. Hill KD, Schwarz JA, Kalogeropoulos AJ, et al. Fear of falling revisited. Arch Phys Med Rehabil 1996;77(10):1025-9.

54. Jorstad EC, Hauer K, Becker C, et al. Measuring the psychological outcomes of falling: a systematic review. J Am Geriatr Soc 2005;53(3):501-10.

55. Tinetti ME, Powell L. Fear of falling and low self-efficacy: a case of dependence in elderly persons. J Gerontol 1993;48 Spec No:35-8.

56. The prevention of falls in later life. A report of the Kellogg International Work Group on the prevention of falls by the elderly. Dan Med Bull 1987;47(12):1397-402.

57. Liddle J, Gilleard C. The emotional consequences of falls for older people and their families. Clin Rehabil 1995;9(2):110-4.

58. Dugan E, Bonds D. Explaining the lack of communication about falls and the fear of falling. Generations 2002;26(4):48-52. 
59. Ward-Griffin C, Hobson S, Melles P, et al. Falls and fear of falling among communitydwelling seniors: the dynamic tension between exercising precaution and striving for independence. Can J Aging 2004;23(4):307-18.

60. Vellas B, Cayla F, Bocquet $H$, et al. Prospective study of restriction of activity in old people after falls. Age Ageing 1987;16(3):189-93.

61. Lawrence RH, Tennstedt SL, Kasten LE, et al. Intensity and correlates of fear of falling and hurting oneself in the next year: baseline findings from a Roybal Center fear of falling intervention. J Aging Health 1998;10(3):267-86.

62. Mann R, Birks Y, Hall J, et al. Exploring the relationship between fear of falling and neuroticism: a cross-sectional study in community-dwelling women over 70. Age Ageing 2006;35(2):143-7.

63. Bruce DG, Devine A, Prince RL. Recreational physical activity levels in healthy older women: The importance of fear of falling. J Am Geriatr Soc 2002;50(1):84-9.

64. Mendes de Leon CF, Seeman TE, Baker DI, et al. Self-efficacy, physical decline, and change in functioning in community-living elders: a prospective study. J Gerontol B Psychol Sci Soc Sci 1996;51(4):S183-90.

65. Delbaere K, Crombez G, Vanderstraeten G, et al. Fear-related avoidance of activities, falls and physical frailty. A prospective community-based cohort study. Age Ageing 2004;33(4):368-73.

66. Stel VS, Pluijm SM, Deeg DJ, et al. A classification tree for predicting recurrent falling in community-dwelling older persons. J Am Geriatr Soc 2003;51(10):1356-64.

67. Lachman ME, Howland J, Tennstedt S, et al. Fear of falling and activity restriction: the survey of activities and fear of falling in the elderly (SAFE). J Gerontol B Psychol Sci Soc Sci 1998;53(1):P43-50.

68. Brouwer B, Musselman K, Culham E. Physical function and health status among seniors with and without a fear of falling. Gerontology 2004;50(3):135-41.

69. Martin FC, Hart D, Spector T, et al. Fear of falling limiting activity in young-old women is associated with reduced functional mobility rather than psychological factors. Age Ageing 2005;34(3):281-7.

70. Andresen EM, Wolinsky FD, Miller JP, et al. Cross-sectional and longitudinal risk factors for falls, fear of falling, and falls efficacy in a cohort of middle-aged African Americans. Gerontologist 2006;46(2):249-57.

71. Walker JE, Howland J. Falls and fear of falling among elderly persons living in the community: occupational therapy interventions. Am J Occup Ther 1991;45(2):119-22.

72. Yardley L. Fear of imbalance and falling. Rev Clin Gerontol 1998;8(1):23-9.

73. Brouwer BJ, Walker C, Rydahl SJ, et al. Reducing fear of falling in seniors through education and activity programs: a randomized trial. J Am Geriatr Soc 2003;51(6):829-34.

74. Means KM, O'Sullivan PS, Rodell DE. Psychosocial effects of an exercise program in older persons who fall. J Rehabil Res Dev 2003;40(1):49-58.

75. Tennstedt S, Howland J, Lachman M, et al. A randomized, controlled trial of a group intervention to reduce fear of falling and associated activity restriction in older adults. J Gerontol B Psychol Sci Soc Sci 1998;53(6):P384-92.

76. Clemson L, Cumming RG, Kendig $H$, et al. The effectiveness of a community-based program for reducing the incidence of falls in the elderly: a randomized trial. J Am Geriatr Soc 2004;52(9):1487-94. 
77. Zhang JG, Ishikawa-Takata K, Yamazaki H, et al. The effects of Tai Chi Chuan on physiological function and fear of falling in the less robust elderly: an intervention study for preventing falls. Arch Gerontol Geriatr 2006;42(2):107-16.

78. Peterson EW. Using cognitive behavioral strategies to reduce fear of falling: A Matter of Balance. Generations 2002;26(4):53-9.

79. Dautzenberg PLJ, Buurman BHT, Loonen AJM, et al. Angst voor vallen op een valpoliklinik geriatrie: een pilotstudy. Tijdschr Gerontol Geriatr 2005;36:138-45.

80. Faber M, Vet-Heijne F. [Care for the elderly with frequent falls: the fall clinic in ZeeuwsVlaanderen, The Netherlands]. Tijdschr Gerontol Geriatr 2005;36(4):168-75.

81. van Marle AM, Schouten H, den Hertog PC. Senioren en valincidenten. Psychosociale gevolgen van een val op lange termijn. Amsterdam: Stichting Consument en Veiligheid, 2005:1-101.

82. van Schoor NM, Asma G, Smit JH, et al. The Amsterdam Hip Protector Study: compliance and determinants of compliance. Osteoporos Int 2003;14(4):353-9.

83. Bosscher R, Raymakers ER, Trompe EA, et al. Angst om te vallen: psychometrische aspecten van Tinetti's Falls Efficacy Scale. Tijdschr Gerontol Geriatr 2005;36:5-10.

84. van Heuvelen MJG, Hochstenbach J, de Greef MHG, et al. Is de Activities-specific Balance Confidence Scale geschikt voor het meten van valangst bij Nederlandse nietgeïnstitutionaliseerde ouderen? Tijdschr Gerontol Geriatr 2005;36:146-54.

85. Kempen GI, Todd CJ, van Haastregt JC, et al. Cross-cultural validation of the Falls Efficacy Scale International (FES-I) in older people: results from Germany, The Netherlands and the UK were satisfactory. Disabil Rehabil 2007;29(2):155-62.

86. Weerdesteyn $\mathrm{V}$, Rijken $\mathrm{H}$, Geurts AC, et al. A five-week exercise program can reduce falls and improve obstacle avoidance in the elderly. Gerontology 2006;52(3):131-41. 



\section{CHAPTER 2}

\section{Prevalence and correlates of fear of falling and associated avoidance of activity in the general population of community-living older people}




\section{Abstract}

Introduction Little is known about prevalence rates and correlates of fear of falling and avoidance of activity due to fear of falling in the general population of communityliving older people.

Objective To assess prevalence rates and study correlates of fear of falling and avoidance of activity due to fear of falling in this population.

Study design and population Cross-sectional study in 4,031 community-living people aged $\geq 70$ years.

Results Fear of falling was reported by $54.3 \%$ and associated avoidance of activity by $37.9 \%$ of our population. Variables independently associated with fear of falling were: higher age ( $\geq 80$ years: Odds Ratio $(O R)=1.79 ; 95 \%$ Confidence Interval $(\mathrm{Cl})$ $=1.49-2.16)$, female gender $(\mathrm{OR}=3.23 ; 95 \% \mathrm{Cl}=2.76-3.79)$, poor perceived general health $(\mathrm{OR}=6.93 ; 95 \% \mathrm{Cl}=4.70-10.21)$ and multiple falls $(\mathrm{OR}=5.72 ; 95 \% \mathrm{Cl}=$ 4.40-7.43). Higher age $(\geq 80$ years: $O R=1.92 ; 95 \% \mathrm{Cl}=1.59-2.32)$, poor perceived general health $(\mathrm{OR}=11.91 ; 95 \% \mathrm{Cl}=8.38-16.95)$ and multiple falls $(\mathrm{OR}=4.64 ; 95 \%$ $\mathrm{Cl}=3.73-5.76)$ were also independently associated with avoidance of activity.

Conclusions Fear of falling and avoidance of activities due to fear of falling were highly prevalent in our sample of community-living older people. Particularly, poor perceived general health showed a strong, independent association with both fear of falling and related avoidance of activity. Findings of our study may help health care professionals to identify people eligible for interventions aimed at reducing fear of falling and activity restriction. 


\section{Introduction}

Fear of falling and avoidance of activity due to fear of falling are common in older people, both in fallers and non-fallers. ${ }^{123}$ In community-living older people, prevalence rates for fear of falling range from 20 to $85 \%^{1-15}$ and from 15 to $55 \%^{12} 1015$ for associated avoidance of activity. Fear of falling and associated avoidance of activity may lead to adverse consequences, like functional decline, ${ }^{7}$ restriction of social participation, ${ }^{2}$ decreased quality of life, ${ }^{3} 7$ and increased risk of falling ${ }^{10}$ and institutionalisation. ${ }^{7}$ Fear of falling is suggested to be a potential health problem of equal importance to a fall ${ }^{7}$ and may also affect society as health care utilisation and costs increase. This underlines the need to study the prevalence of fear of falling and associated avoidance of activity, and to identify those fearful and avoidant older people in order to facilitate recommendation of prevention strategies.

Current literature on fear of falling and associated avoidance of activity shows several limitations. First, prevalence rates are often based on samples of communityliving older people, yet, most of these samples do not represent the general population of community-living older people. ${ }^{2614-16}$ In addition, in most studies prevalence according to subgroups could not be studied due to relatively small subgroups. Second, factors independently related to fear of falling and particularly for avoidance of activity due to fear of falling are understudied. To identify the appropriate population for prevention strategies knowledge of these factors is important.

This cross-sectional study was performed in a random sample of the general population of community-living older people in The Netherlands and aimed to: (a) assess the prevalence of fear of falling and avoidance of activity due to fear of falling and (b) study correlates of fear of falling and avoidance of activity due to this fear.

\section{Methods}

Study participants were older people living in two urban areas in The Netherlands. Between November 2002 and July 2003, local municipal registry offices selected a random sample of 7,431 community-living people aged $\geq 70$ years. Selected persons received a short, posted questionnaire. If the questionnaire was not returned in a fortnight, a reminder to return the questionnaire was sent. This study was approved by the Medical Ethics Committee of Maastricht University/Academic Hospital Maastricht. ${ }^{17}$

The questionnaire assessed fear of falling ("Are you afraid of falling?") and associated avoidance of activity ("Do you avoid certain activities due to fear of falling?"). Participants indicated the frequency (never, almost never, sometimes, often or very often) of experiencing this fear or avoiding activities. For the analyses in which fear of falling and associated avoidance of activity (as of here called avoidance of activity) were applied as dependent variables, this frequency was dichotomised (sometimes, often and very often versus almost never and never). In addition, several socio-demographic and health-related variables were assessed: age (aged 70 to 74 , 75 to 79 or $\geq 80$ years), gender (male and female), living situation (alone and not alone), educational level based on completed education and completed professional 
courses during lifetime (low, middle and high), ${ }^{18} 19$ perceived general health (good, fair and poor) 2021 and falls in the past 6 months (never, once and more than once).

\section{Statistical methods}

To test for differences in age and gender between non-responders and responders to the questionnaire, the Mann-Whitney test statistic and chi-square test, respectively, were used. Next, two sets of univariate analyses were performed using chi-square tests. First, analyses were performed to identify associations between fear of falling and socio-demographic and health-related variables. Second, similar analyses were performed with avoidance of activity instead of fear of falling. Lastly, three series of multivariate logistic regression analyses were performed. First, an analysis was performed to test which of the socio-demographic and health-related variables was independently associated with fear of falling (model 1). Second, a similar strategy was applied for avoidance of activity instead of fear of falling (model 2). Third, an analysis was performed to study the association of these variables with avoidance of activity, independent of the measurement of fear of falling (model 3). For this purpose, fear of falling, which may be considered as a mediator between the selected variables and avoidance of activity, was added to model 2. Those participants reporting never afraid of falling were excluded from this analysis as avoidance of activity due to fear of falling is not applicable to these participants. All socio-demographic and health-related variables were included in the three models, since $p$ values of $<.01$ were obtained at the univariate analyses for the appropriate dependent variables. Odds ratios (OR) and 95\% confidence intervals $(\mathrm{Cl})$ were calculated.

All analyses were conducted using SPSS for Windows version 12.0 (SPSS, Inc. Chicago, IL).

\section{Results}

Of the 7,431 older people who were approached, 4,376 responded to the questionnaire (response rate $58.9 \%$ ). A total of 4,031 questionnaires were included in the analyses since 345 were incomplete. Non-responders and those excluded ( $n=$ 3,400 ) differed significantly from responders regarding age (mean age 78.1, standard deviation $(S D)=5.5$ versus mean age $77.1, S D=4.9 ; p \leq 01)$ and gender $(62.5$ versus $59.9 \%$ female; $p=.02$ ).

Table 1 summarises participants' characteristics and correlates of fear of falling and avoidance of activity. Of the participants, $39.8 \%$ were aged 70 to 74 years, $40.1 \%$ were males, $44.0 \%$ lived alone and $60.7 \%$ had a low educational level. Over half of the participants rated their general health as good and $67.4 \%$ reported no falls in the past 6 months.

Prevalence rates for fear of falling and avoidance of activity are presented in Table 2 . In total, $54.3 \%$ of the participants reported fear of falling (sometimes, often and very often) and $37.9 \%$ reported avoidance of activity (sometimes, often and very often). Of those experiencing fear of falling two thirds (65.5\%) reported avoiding activities (data not shown). 
Table 1 Participants' characteristics and correlates of fear of falling and avoidance of activity in communityliving older people $(\mathrm{N}=4,031)^{*}$

\begin{tabular}{|c|c|c|c|c|c|c|}
\hline \multirow[b]{2}{*}{ Variables } & \multirow{2}{*}{\multicolumn{2}{|c|}{ Number (\%) }} & \multicolumn{2}{|c|}{ Fear of falling, $\%^{\dagger}$} & \multicolumn{2}{|c|}{ Avoidance of activity, $\%{ }^{\dagger}$} \\
\hline & & & Yes & No & Yes & No \\
\hline \multicolumn{7}{|l|}{ Age } \\
\hline $70-74$ years & 1,606 & (39.8) & 45.8 & 54.2 & 29.9 & 70.1 \\
\hline $75-79$ years & 1,357 & (33.7) & 55.2 & 44.8 & 37.7 & 62.3 \\
\hline$\geq 80$ years & 1,068 & (26.5) & 65.6 & 34.4 & 50.5 & 49.5 \\
\hline \multicolumn{7}{|l|}{ Gender } \\
\hline male & 1,615 & $(40.1)$ & 37.0 & 63.0 & 25.8 & 74.2 \\
\hline female & 2,416 & (59.9) & 65.7 & 34.3 & 46.1 & 53.9 \\
\hline \multicolumn{7}{|l|}{ Living situation } \\
\hline alone & 1,774 & $(44.0)$ & 62.2 & 37.8 & 44.5 & 55.5 \\
\hline not alone & 2,257 & $(56.0)$ & 48.0 & 52.0 & 32.8 & 67.2 \\
\hline \multicolumn{7}{|l|}{ Educational level } \\
\hline low & 2,447 & $(60.7)$ & 58.9 & 41.1 & 43.2 & 56.8 \\
\hline middle & 1,016 & (25.2) & 47.9 & 52.1 & 30.3 & 69.7 \\
\hline high & 568 & $(14.1)$ & 45.4 & 54.6 & 29.0 & 71.0 \\
\hline \multicolumn{7}{|c|}{ Perceived general health } \\
\hline good & 2,116 & (52.5) & 38.8 & 61.2 & 19.7 & 80.3 \\
\hline fair & 1,703 & $(42.2)$ & 69.9 & 30.1 & 56.0 & 44.0 \\
\hline poor & 212 & (5.3) & 82.1 & 17.9 & 75.5 & 24.5 \\
\hline \multicolumn{7}{|c|}{ Falls in the past 6 months } \\
\hline never & 2,716 & (67.4) & 43.8 & 56.2 & 28.5 & 71.5 \\
\hline once & 703 & (17.4) & 66.3 & 33.7 & 43.4 & 56.6 \\
\hline more than once & 612 & (15.2) & 86.8 & 13.2 & 73.7 & 26.3 \\
\hline
\end{tabular}

The univariate analyses (see Table 1) showed that all socio-demographic and healthrelated variables were associated with both fear of falling and avoidance of activity.

Table 3 presents multivariate associations between fear of falling and avoidance of activity and socio-demographic and health-related variables. Significant independent associations with experiencing fear of falling and avoidance of activity were found for: higher age, female gender, worsened perceived general health and increased number of falls. For example for fear of falling (model 1), compared to participants aged 70 to 74 years, those aged 75 to 79 were $40 \%$ more likely to experience fear of falling $(O R=1.40 ; 95 \% \mathrm{Cl}=1.18-1.65)$ and compared to men, women were over three times as likely to experience fear of falling $(\mathrm{OR}=3.23 ; 95 \%$ $\mathrm{Cl}=2.76-3.79$ ). For example for avoidance of activity (model 2), compared to those indicating their general health as good, those indicating their general health as poor 
Table 2 Prevalence of fear of falling and avoidance of activity in community-living older people $(\mathrm{N}=4,031)$

\begin{tabular}{lrlll} 
& \multicolumn{2}{c}{ Fear of falling } & \multicolumn{2}{c}{ Avoidance of activity } \\
\hline \multicolumn{2}{c}{ Number $(\%)$} & \multicolumn{3}{c}{ Number (\%) } \\
\hline very often & 216 & $(5.4)$ & 193 & $(4.8)$ \\
often & 407 & $(10.1)$ & 396 & $(9.8)$ \\
sometimes & 1,563 & $(38.8)$ & 941 & $(23.3)$ \\
almost never & 746 & $(18.5)$ & 600 & $(14.9)$ \\
never & 1,099 & $(27.3)$ & 1,901 & $(47.2)$ \\
\hline
\end{tabular}

were nearly 12 times as likely to avoid activities ( $\mathrm{OR}=11.91 ; 95 \% \mathrm{Cl}=8.38-16.95)$ and compared to those who had not fallen, those who had fallen more than once were over four times $(\mathrm{OR}=4.64 ; 95 \% \mathrm{Cl}=3.73-5.76)$ as likely to avoid activities. Living situation and educational level were not independently associated with fear of falling or avoidance of activity.

When fear of falling was added as an additional variable (model 3; Table 3), odds ratios of all variables that showed significance in model 2 decreased. Nevertheless, the association for the highest age group ( $\geq 80$ years), fair and poor perceived general health and multiple falls with avoidance of activities remained statistically significant.

\section{Discussion}

In our sample of 4,031 older people randomly selected from the general population of community-living older people, 54\% reported fear of falling and 38\% reported avoiding activity due to fear of falling. These prevalence rates are comparable to prevalence rates reported in several studies. ${ }^{16810-12} 14$ Some studies, however, reported either lower or higher prevalence rates. ${ }^{2-5} 71315$ Variations in prevalence rates may be due to differences in population characteristics, like age, fall history and frailty, and applied measures. ${ }^{10} 14$

With regard to identifying variables related to fear of falling, the variables we found to be univariately associated with this fear correspond to those reported by Arfken and colleagues $^{3}$ and Howland and colleagues ${ }^{6}$ : higher age, female gender, fair and poor perceived general health, and having experienced one or more falls. In our multivariate analysis the independent association between these variables and fear of falling remained. Arfken and colleagues ${ }^{3}$ showed similar results, but Howland and colleagues ${ }^{6}$ only found female gender and falls to be independently related to fear of falling. These latter findings were supported in a study among community-living older people using home care services. ${ }^{14}$ In another study among community-living older females contacted for participation in a hip protector trial, living alone, poor general health and history of falling showed to be associated with fear of falling, however, higher age was not associated with fear of falling. ${ }^{22}$ In accordance with our findings, living situation and educational level were not associated with experiencing fear of falling in other studies. ${ }^{26-8}$ Thus, the evidence for 
Table 3 Multivariate associations between fear of falling and avoidance of activity and socio-demographic and health-related variables in community-living older people*

Model 1

Fear of falling as dependent variable
Model 2 Avoidance of activity Avoidance of activity as dependent variable as dependent variable ${ }^{\dagger}$

\begin{tabular}{|c|c|c|c|c|c|c|}
\hline \multirow[b]{2}{*}{ Variables } & \multicolumn{2}{|c|}{$(\mathrm{N}=4,031)$} & \multicolumn{2}{|c|}{$(\mathrm{N}=4,031)$} & \multicolumn{2}{|c|}{$(\mathrm{N}=2,932)$} \\
\hline & OR & $(95 \% \mathrm{Cl})$ & OR & $(95 \% \mathrm{Cl})$ & OR & $(95 \% \mathrm{Cl})$ \\
\hline \multicolumn{7}{|l|}{ Age } \\
\hline 70 - 74 years & 1.00 & & 1.00 & & 1.00 & \\
\hline $75-79$ years & 1.40 & $(1.18-1.65)$ & 1.30 & $(1.09-1.56)$ & 1.14 & $(0.92-1.41)$ \\
\hline$\geq 80$ years & 1.79 & $(1.49-2.16)$ & 1.92 & $(1.59-2.32)$ & 1.56 & $(1.24-1.95)$ \\
\hline \multicolumn{7}{|l|}{ Gender } \\
\hline male & 1.00 & & 1.00 & & 1.00 & \\
\hline female & 3.23 & $(2.76-3.79)$ & 2.27 & $(1.92-2.69)$ & 1.05 & $(0.85-1.29)$ \\
\hline \multicolumn{7}{|l|}{ Living situation } \\
\hline alone & 1.00 & & 1.00 & & 1.00 & \\
\hline not alone & 0.93 & $(0.79-1.08)$ & 0.92 & $(0.79-1.08)$ & 0.98 & $(0.81-1.18)$ \\
\hline \multicolumn{7}{|l|}{ Educational level } \\
\hline low & 1.00 & & 1.00 & & 1.00 & \\
\hline middle & 1.05 & $(0.88-1.24)$ & 0.87 & $(0.73-1.05)$ & 0.74 & $(0.60-0.93)$ \\
\hline high & 1.04 & $(0.84-1.29)$ & 0.88 & $(0.70-1.11)$ & 0.72 & $(0.54-0.95)$ \\
\hline \multicolumn{7}{|c|}{ Perceived general health } \\
\hline good & 1.00 & & 1.00 & & 1.00 & \\
\hline fair & 3.19 & $(2.75-3.71)$ & 4.42 & $(3.79-5.15)$ & 2.92 & $(2.43-3.52)$ \\
\hline poor & 6.93 & $(4.70-10.21)$ & 11.91 & $(8.38-16.95)$ & 5.70 & $(3.57-9.12)$ \\
\hline \multicolumn{7}{|c|}{ Falls in the past 6 months } \\
\hline never & 1.00 & & 1.00 & & 1.00 & \\
\hline once & 2.28 & $(1.89-2.75)$ & 1.69 & $(1.40-2.04)$ & 1.09 & $(0.87-1.36)$ \\
\hline more than once & 5.72 & $(4.40-7.43)$ & 4.64 & $(3.73-5.76)$ & 1.97 & $(1.52-2.54)$ \\
\hline \multicolumn{7}{|l|}{ Fear of falling } \\
\hline almost never & - & - & - & - & 1.00 & \\
\hline sometimes & - & - & - & - & 7.15 & $(5.59-9.13)$ \\
\hline often & - & - & - & - & 24.61 & $(17.02-35.59)$ \\
\hline very often & - & - & - & - & 63.61 & (33.63-120.32) \\
\hline
\end{tabular}

Note: All variables were entered simultaneously into each multivariate logistic regression model.

OR = odds ratio; $95 \% \mathrm{Cl}=95 \%$ confidence interval.

* The dependent variables fear of falling and avoidance of activity comprise participants reporting sometimes, often and very often experiencing fear of falling or avoidance of activity due to fear of falling.

† The measurement of fear of falling was added to this model.

female gender, fair and poor perceived general health, and falls as independent correlates of fear of falling appears strong, while living situation and education level should not be regarded as factors independently related to fear of falling. 
With regard to identifying variables independently related to avoidance of activity, we found significant associations with higher age, female gender, fair and poor perceived general health, and multiple falls. Particularly, fair and poor perceived general health and multiple falls were strongly associated with avoidance of activity. Educational level and living situation were not associated with avoidance of activity when other variables were taken into account. Regarding female gender, poor self-rated health and multiple falls as correlates of avoidance of activity, our results correspond with previous findings, ${ }^{14}$ however, they do not correspond with results in several other studies. ${ }^{611} 23$ These variations in findings may be explained by differences in population characteristics or settings. Our results correspond to previous findings in that living situation and educational level were not independently associated with avoidance of activity and add that higher age may be regarded as a factor independently related to avoidance of activity in community-living older people.

Our findings regarding avoidance of activity remained fairly similar when fear of falling was entered into the logistic model. Although sometimes, often and very often experiencing fear of falling were strongly associated with avoidance of activity, higher age ( $\geq 80$ years), fair and poor perceived health, and multiple falls remained independently associated with avoidance of activity in community-living older people. This implies that interventions aimed at reducing avoidance of activity should not focus on fear of falling alone, but on other modifiable factors, like falls, as well.

There are limitations that may have affected the results of our study. First, although we approached a representative sample of the general population of community-living older people, about $40 \%$ did not respond to the questionnaire. Non-responders and those excluded showed to be slightly older than responders and were more likely to be female. Consequently our prevalence rates may be somewhat underestimated as our study showed that fear of falling and avoidance of activity are more common in people of high age and female gender. Due to strict policies of municipal registry offices regarding people's privacy, no additional contact with non-responders of our study was permitted. However, if permitted, we recommend non-response research in future studies to gain insight into non-responders regarding general characteristics and levels of fear of falling and avoidance of activity. We expect the impact of our non-response on the correlates of fear of falling and avoidance of activity to be limited not only as our associations showed to be similar to those in other studies but also as the impact of non-response on studied associations showed to be quite small. ${ }^{24}$ Second, prevalence rates of fear of falling and avoidance of activity may also have been underreported since reluctance to acknowledge fear of falling among older people has been observed. ${ }^{12} 25$ Third, we assessed both fear of falling and avoidance of activity with a one-item question, which may not be the most optimal way to measure a construct. The one-item fear of falling question, however, has been found to correlate significantly with the Falls Efficacy Scale International, ${ }^{26}$ a 16 -item measure that assesses one's concern regarding falls in both easy and more complex physical and social activities. ${ }^{27}$ Lastly, like other cross-sectional studies, the design of our study limits interpretation of the results with regard to causality between particularly health-related variables on the one hand and fear of falling or avoidance of activity on the other. In a prospective study, ${ }^{10}$ fear of falling showed to be an independent predictor for falls and vice versa. 
It seems plausible that this might also apply to avoidance of activity, and, in addition, to both fear of falling and avoidance of activity and perceived general health. Further research is warranted to study the causal relationship between fear of falling or avoidance of activity and perceived general health.

This study has several strengths as well. First, our study population was randomly selected from the general population of community-living older people. Furthermore, the large sample of our study enabled us to create substantial subgroups for the different socio-demographic and health-related variables. Finally, our findings are important for practice, they may help health care professionals recognise fear of falling and avoidance of activity in older people.

In conclusion, our study shows that fear of falling and avoidance of activity are highly prevalent among community-living older people in The Netherlands. The independent associations found in this large sample support several findings in other studies. Particularly, strong associations were found for fair and poor perceived general health, and multiple falls with fear of falling and associated avoidance of activity. Our study adds the new insight that higher age also showed to be independently related to avoidance of activity. It also adds that both fair and poor perceived general health, and multiple falls in the past 6 months may be important modifiable factors that, independently from fear of falling, may need to be taken into consideration when aiming to reduce avoidance of activity. The findings of the current study are important for prevention purposes and may help health care professionals to identify people at risk for fear of falling and associated avoidance of activity. Health care professionals are vital links in the process of recognising fearful and avoidant older people and referring them to interventions. We therefore recommend they inquire after fear of falling and associated avoidance of activity in patients at risk, for instance patients in poor health. Subsequently, fearful and avoidant persons could be referred to interventions aimed at reducing fear of falling and increasing activity, such as a cognitive behavioural intervention, ${ }^{28}$ a tai chi intervention ${ }^{29}$ or a home-based exercise intervention ${ }^{30}$ for community-living older people.

\section{Acknowledgements}

We would like to thank Vonca Schaffers, Laury Quaedackers and the municipal registry offices of Maastricht and Heerlen for their assistance in this study. This research was conducted as part of a randomised controlled trial that has been funded by ZonMw The Netherlands Organisation for Health Research and Development (grant 014-91052), the Care and Public Health Research Institute, and the Faculty of Health Sciences of the Maastricht University. 


\section{References}

1. Tinetti ME, Speechley M, Ginter SF. Risk factors for falls among elderly persons living in the community. N Engl J Med 1988;319(26):1701-7.

2. Howland J, Peterson EW, Levin WC, et al. Fear of falling among the communitydwelling elderly. J Aging Health 1993;5(2):229-43.

3. Arfken $\mathrm{CL}$, Lach HW, Birge SJ, et al. The prevalence and correlates of fear of falling in elderly persons living in the community. Am J Public Health 1994;84(4):565-70.

4. Underwood MR, Parsons S, Eldridge SM, et al. Asking older people about fear of falling did not have a negative effect. J Clin Epidemio/ 2006;59(6):629-34.

5. Vellas BJ, Wayne SJ, Romero $L$, et al. Fear of falling and restriction of mobility in elderly fallers. Age Ageing 1997;26(3):189-93.

6. Howland J, Lachman ME, Peterson EW, et al. Covariates of fear of falling and associated activity curtailment. Gerontologist 1998;38(5):549-55.

7. Cumming RG, Salkeld G, Thomas M, et al. Prospective study of the impact of fear of falling on activities of daily living, SF-36 scores, and nursing home admission. I Gerontol A Biol Sci Med Sci 2000;55(5):M299-305.

8. Drozdick LW, Edelstein BA. Correlates of fear of falling in older adults who have experienced a fall. J Clin Geropsychol 2001;7(1):1-13.

9. Kressig RW, Wolf SL, Sattin RW, et al. Associations of demographic, functional, and behavioral characteristics with activity-related fear of falling among older adults transitioning to frailty. J Am Geriatr Soc 2001;49(11):1456-62.

10. Friedman SM, Munoz B, West SK, et al. Falls and fear of falling: which comes first? A longitudinal prediction model suggests strategies for primary and secondary prevention. J Am Geriatr Soc 2002;50(8):1329-35.

11. Murphy SL, Williams CS, Gill TM. Characteristics associated with fear of falling and activity restriction in community-living older persons. J Am Geriatr Soc 2002; 50(3):516-20.

12. Yardley L, Smith H. A prospective study of the relationship between feared consequences of falling and avoidance of activity in community-living older people. Gerontologist 2002;42(1):17-23.

13. Li F, Fisher KJ, Harmer $P$, et al. Fear of falling in elderly persons: association with falls, functional ability, and quality of life. J Gerontol B Psychol Sci Soc Sci 2003; 58(5):P28390.

14. Fletcher PC, Hirdes JP. Restriction in activity associated with fear of falling among community-based seniors using home care services. Age Ageing 2004;33(3): 273-9.

15. Wilson MM, Miller DK, Andresen EM, et al. Fear of falling and related activity restriction among middle-aged African Americans. J Gerontol A Biol Sci Med Sci 2005; 60(3):35560.

16. Powell LE, Myers AM. The Activities-specific Balance Confidence (ABC) Scale. J Gerontol A Biol Sci Med Sci 1995;50a(1):M28-34.

17. Zijlstra GA, van Haastregt JC, van Eijk JT, et al. Evaluating an intervention to reduce fear of falling and associated activity restriction in elderly persons: design of a randomised controlled trial [ISRCTN43792817]. BMC Public Health 2005;5(1):26. 
18. CBS. Standaard Onderwijsindeling 1998. Editie 2001/'02. Voorburg/Heerlen: Centraal Bureau voor de Statistiek, 2001.

19. UNESCO. International Standard Classification of Education, ISCED. Paris: UNESCO, 1997.

20. Stewart AL, Hays RD, Ware JE, Jr. The MOS short-form general health survey. Reliability and validity in a patient population. Med Care 1988;26(7):724-35.

21. Kempen $\mathrm{Gl}$, Brilman El, Heyink JW, et al. Het meten van de algemene gezondheidstoestand met de MOS Short-Form General Health Survey (SF-20): een handleiding. Groningen: Noordelijk Centrum voor Gezondheidsvraagstukken, Rijksuniversiteit Groningen, 1995.

22. Mann R, Birks Y, Hall J, et al. Exploring the relationship between fear of falling and neuroticism: a cross-sectional study in community-dwelling women over 70. Age Ageing 2006;35(2):143-7.

23. Martin FC, Hart D, Spector T, et al. Fear of falling limiting activity in young-old women is associated with reduced functional mobility rather than psychological factors. Age Ageing 2005;34(3):281-7.

24. Kempen Gl, van Sonderen E. Psychological attributes and changes in disability among low-functioning older persons: does attrition affect the outcomes? I Clin Epidemiol 2002;55(3):224-9.

25. Liddle J, Gilleard C. The emotional consequences of falls for older people and their families. Clin Rehabil 1995;9(2):110-4.

26. Kempen GI, Todd CJ, van Haastregt JC, et al. Cross-cultural validation of the Falls Efficacy Scale International (FES-I) in older people: results from Germany, The Netherlands and the UK were satisfactory. Disabil Rehabil 2007;29(2):155-62.

27. Yardley L, Beyer N, Hauer K, et al. Development and initial validation of the Falls Efficacy Scale-International (FES-I). Age Ageing 2005;34(6):614-9.

28. Tennstedt S, Howland J, Lachman M, et al. A randomized, controlled trial of a group intervention to reduce fear of falling and associated activity restriction in older adults. J Gerontol B Psychol Sci Soc Sci 1998;53(6):P384-92.

29. Li F, Harmer P, Fisher KJ, et al. Tai chi and fall reductions in older adults: a randomized controlled trial. J Gerontol A Biol Sci Med Sci 2005;60(2):187-94.

30. Robertson MC, Devlin N, Gardner MM, et al. Effectiveness and economic evaluation of a nurse delivered home exercise programme to prevent falls. 1: Randomised controlled trial. BMJ 2001;322(7288):697-701. 



\section{CHAPTER 3}

\section{Interventions to reduce fear of falling in community-living older people: a systematic review}




\section{Abstract}

Objective The objective was to assess which interventions effectively reduce fear of falling in community-living older people.

Methods An extensive search for relevant literature comprised a database search of PubMed, EMBASE, PsycINFO, and Central Register of Controlled Trials, expert consultation and manually searching reference lists from potentially relevant papers. Randomised controlled trials that assessed fear of falling in community-living older people were included. Two independent reviewers extracted data from full papers on study characteristics, methodological quality, outcomes and process characteristics of the intervention.

Results The search identified 599 abstracts and 19 papers met the inclusion criteria. Seven of those papers were identified using expert consultation. Fifty-five percent of all validity items and $39 \%$ of process characteristic items were fulfilled across the 19 trials. Twelve of the 19 papers were of higher methodological quality. In 11 of these trials fear of falling was lower in the intervention group than in the control group. Interventions that showed effectiveness were fall-related multifactorial programmes $(n=5)$, tai chi interventions $(n=3)$, exercise interventions $(n=2)$ and a hip protector intervention $(n=1)$. Three of these interventions explicitly aimed to reduce fear of falling.

Conclusions Several interventions, including interventions not explicitly aimed at fear of falling, showed a reduction of fear of falling in community-living older people. Limited but fairly consistent findings in trials of higher methodological quality showed that home-based exercise and fall-related multifactorial programmes and community-based tai chi delivered in group format have been effective in reducing fear of falling in community-living older people. 


\section{Introduction}

Approximately half of the community-living older population experiences fear of falling. ${ }^{1-4}$ Because of a lack of studies that allow conclusions regarding causality in the research field of fear of falling, little evidence is available on what causes this fear. The experience of a recent fall is a known cause for the development of fear of falling, but fear of falling is also prevalent in non-fallers. ${ }^{5}$ Therefore, it is plausible that factors related to the process of aging, such as physical frailty, contribute to the development of fear of falling as well. Several studies have indicated that people who are afraid of falling appear to enter a debilitating spiral of loss of confidence, restriction of physical activities and social participation, physical frailty, falls and loss of independence. ${ }^{4-10}$ In addition to the adverse consequences of fear of falling for those suffering from it, there are consequences for the public expenditure, because health care utilisation increases. ${ }^{9}$ It is therefore important to reduce fear of falling by reversing the downward spiral by intervening in either factors in the spiral, such as increasing physical functioning, ${ }^{10}$ or in predictors of those factors, such as improved medication use. ${ }^{5}$

In the past decade, a substantial number of studies have assessed the effectiveness of interventions on fear of falling. In these studies, interventions explicitly aimed and interventions not explicitly aimed at reducing fear of falling were evaluated but an overview of these studies is lacking. Insight into which kind of interventions effectively reduce or prevent an increase in fear of falling in older people and whether these interventions were conducted according to protocol is essential for implementation and future research. Therefore, a systematic review was conducted to assess the methodological quality of trials evaluating interventions that could reduce fear of falling in community-living older people, the kind of interventions that effectively reduce fear of falling in this population, and the extent to which process characteristics of these interventions are described. Because well-conducted randomised controlled trials are considered the most appropriate studies to assess the effectiveness of interventions, ${ }^{11}$ only randomised controlled trials were included.

\section{Methods}

\section{Fear of falling}

Measures developed to assess psychological outcomes of falling have applied different conceptualisations, for instance afraid of falling, falls self-efficacy, concern about falling and balance confidence. ${ }^{12}$ In this review the term 'fear of falling' is used as an umbrella term for these concepts.

\section{Search strategy}

On January 3 2006, PubMed (1966-2005), the Cochrane Central Register of Controlled Trials (The Cochrane Library Issue 4, 2005), EMBASE (1989-2005) and PsycINFO (18062005) were searched. The Cochrane Collaboration search strategy for randomised controlled trials was used. ${ }^{11}$ This was restricted to older people by using "aged" as a 
Medical Subheading (MeSH) term and combined with the following terms: "balance confidence", "fear of falling", "falls self-efficacy", "concern about falling", "afraid of falling" and "worry about falling". Additional studies were identified by consulting 14 experts, including members of the Prevention of Falls Network Europe (www.profane.eu.org), ${ }^{13}$ and hand-searching reference lists from potentially relevant papers. The search was not restricted by language or publication status.

\section{Selection criteria}

Randomised controlled trials with fear of falling as an outcome were included. To reduce the heterogeneity of the study populations, only trials that were targeted at the general population of community-living older people with a mean age of 65 years or older were included. Studies targeted at people with a specific medical condition were excluded. No restrictions were imposed regarding type of intervention. Interventions explicitly aimed and interventions not explicitly aimed to reduce fear of falling were included.

\section{Collected data}

Methodological quality of trials was assessed using an adaptation of the Cochrane Back Review Group list of criteria (Table 1). ${ }^{14}$ Four items were disregarded, because they were used as inclusion criteria (random allocation and relevance of outcome measure) or were not applicable to the evaluated interventions (blinding of participant and care provider). The criteria list comprised five descriptive, two statistical and eight validity items. Each item was scored "+" if the criterion was fulfilled, "-" if the criterion was not fulfilled, and "?" if the information was not provided or was unclear. Scores on validity items ranged from 0 to 8 per trial. Trials with at least four fulfilled validity items were considered of "higher methodological quality". ${ }^{15}$ In addition, general characteristics of the paper, outcomes of the trial and process characteristics of the intervention were extracted (Table 1). This qualitative analysis was performed because of significant clinical heterogeneity between trials regarding outcome measures and intervention characteristics.

\section{Data extraction}

Titles and abstracts resulting from the database search were independently reviewed (GZ and $J v H)$. Full papers were obtained for potentially relevant studies and trials resulting from expert consultation and manually searching reference lists. Data from all relevant papers were independently extracted $(\mathrm{GZ}$ and $\mathrm{JvH})$, except for one of which one of the authors (JVH) was the first author. Two other authors reviewed this paper (GZ and GK). If information about general characteristics and outcomes was absent from the original paper, attempts were undertaken to obtain complete information from the authors. Reviewers were not blinded to authors' names or institutions or journal of publication. Disagreement was resolved by consensus or a third party (EvR). 
Table 1 Extracted data from the selected full papers

\section{General characteristics}

author, year of publication, country

main objective trial

included population

intervention details (e.g., type of intervention, dose, duration, setting, format)

Methodological quality of trials (criteria list according to Cochrane Back Review Group ${ }^{14}$ )

\section{Descriptive items}

1. Were eligibility criteria clearly specified?

2. Were index and control interventions explicitly described?

3. Was there a description of whether adverse effects had or had not occurred?

4. Was a short-term follow-up measurement (directly after the intervention) of fear of falling performed?

5. Was a long-term follow-up measurement ( $\geq 6$ months after the intervention) of fear of falling performed?

Statistical items

6. Was the sample size for each group described?

7. Were point estimates and measures of variability presented for fear of falling?

Validity items

8. Was treatment allocation concealed?

9. Were groups similar at baseline regarding age, gender and fear of falling?

10. Were co-interventions avoided or comparable?

11. Was compliance acceptable in all groups?

12. Was the outcome assessor blinded to the intervention?

13. Was the withdrawal/dropout rate acceptable (maximum of $20 \%$ for short-term follow-up and $30 \%$ for long-term follow-up)?

14. Was timing of the outcome assessment in both groups comparable?

15. Did the analysis include an intention-to-treat analysis?

Results of the trial

fear-of-falling outcome measure

baseline and follow-up details: number of participants, time of follow-up and results of the analyses

Process characteristics of the intervention

Is information provided on:

a. Characteristics of the facilitator

b. Compliance of participants to the intervention

c. Dropout during the intervention

d. Reasons for dropout during the intervention

e. Performance of intervention according to protocol

f. Feasibility of the intervention

g. Recommendations for improving the intervention 


\section{Results}

Five hundred ninety-nine abstracts were identified, of which 106 were considered potentially relevant after screening for inclusion criteria; 87 were excluded (Figure 1). Of the 19 included trials, ${ }^{16-34} 11$ resulted from searching databases, seven from consulting experts, and one from manually searching reference lists. Five abstracts were excluded, because they reported on a data set of a trial included in the review. ${ }^{35-39}$

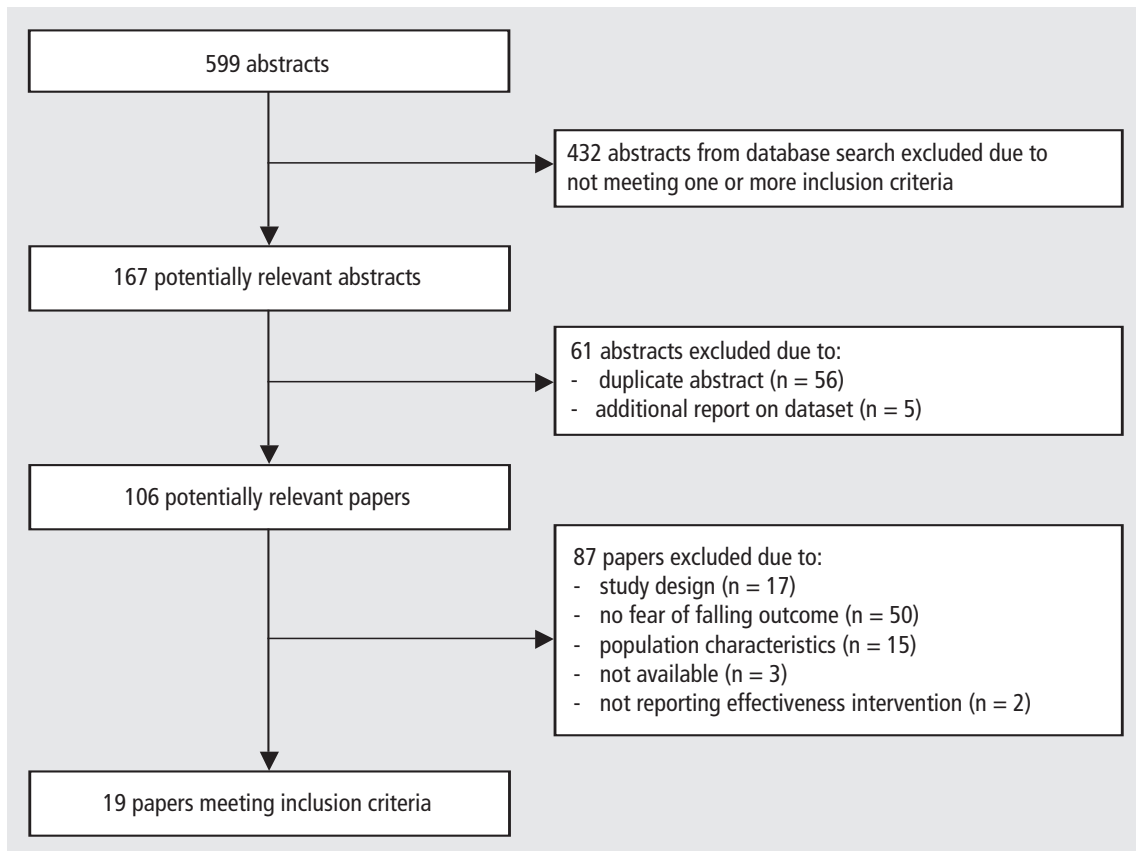

Figure 1 Progress of search for relevant trials

Results of the methodological quality assessment are shown in Table 2. The observed total validity score on fulfilled criteria ranged from 0 to 8 , with a median of 4 . Twelve trials fulfilled at least four criteria and were considered to be of higher methodological quality. ${ }^{16-19,24,27-30,32-34}$ Of all eight validity items across the 19 trials ( $n=152$ ), 55\% (84/152) were fulfilled, 7\% (10/152) were not fulfilled, and 38\% (58/152) were not provided or unclear. Most papers provided sufficient information on similarity between groups at baseline and withdrawal at follow-up, although reporting whether, for instance, the outcome assessor was blinded or treatment allocation was concealed, was less common.

Table 3 presents general characteristics and outcomes of the trials. Trials differed substantially regarding intervention characteristics and outcome measures. Within the 19 trials, eight fall-related multifactorial interventions, ${ }^{19,20,26,28-30,33}$ three tai chi 
Table 2 Methodological quality of included trials*

\begin{tabular}{|c|c|c|c|c|c|c|c|}
\hline \multirow[b]{2}{*}{ Study } & Descriptive & Statistical & \multicolumn{3}{|c|}{ Validity } & \multicolumn{2}{|c|}{$\begin{array}{c}\text { Total } \\
\text { per trial }^{\dagger}\end{array}$} \\
\hline & 12345 & 67 & 8910 & 1112 & 131415 & $?$ & + \\
\hline Barnett, 2003, Australia16 & ++-- & +- & $++?$ & ++ & +++ & 1 & 7 \\
\hline Cameron, 2000, Australia ${ }^{17}$ & $+-\cdot-$ & ++ & $++?$ & ++ & +++ & 1 & 7 \\
\hline Campbell, 1997, New Zealand ${ }^{18}$ & ++-+ & ++ & $++?$ & $?+$ & +++ & 2 & 6 \\
\hline Clemson, 2004, Australia ${ }^{19}$ & ++-+ & ++ & $++?$ & ++ & +++ & 1 & 7 \\
\hline Gallagher, 1996, Canada20 & ++-+ & +- & $?+?$ & $-\quad-$ & $++?$ & 3 & 3 \\
\hline Hamel, 2005, Canada 21 & +- ? ? & $?-$ & ? ? ? & $? ?$ & ? ? ? & 8 & 0 \\
\hline Hinman, 2002, USA 22 & ++-+- & ++ & $?-?$ & ? ? & $++?$ & 5 & 2 \\
\hline Huang, 2004, Taiwan²3 & +--+ & +- & $?+?$ & $?-$ & $+? ?$ & 5 & 2 \\
\hline Li, 2005, USA ${ }^{24}$ & ++++ & ++ & $++?$ & ++ & +++ & 1 & 7 \\
\hline Nitz, 2004, Australia25 & ++++ & +- & $+? ?$ & $?+$ & $-\quad+?$ & 4 & 3 \\
\hline Reinsch, 1992, USA 26 & ++-+ & ++ & $?+?$ & $? ?$ & $-\quad+?$ & 5 & 2 \\
\hline Robertson, 2001, New Zealand ${ }^{27}$ & ++++ & ++ & +++ & ++ & +++ & 0 & 8 \\
\hline Tennstedt, 1998, USA 28 & ++-++ & +- & $?+?$ & $-\quad+$ & +++ & 2 & 5 \\
\hline Tinetti, 1994, USA 29 & +++-+ & ++ & $++?$ & ++ & $+? ?$ & 3 & 5 \\
\hline Van Haastregt, 2000, Netherlands 30 & ++-+ & ++ & $?+?$ & $-?$ & +++ & 3 & 4 \\
\hline Wolf, 2001, Netherlands 31 & ++-+ & ++ & $++?$ & $? ?$ & $-?+$ & 4 & 3 \\
\hline Wolf, 1996, USA 32 & ++-+ & ++ & $++?$ & $+?$ & $++?$ & 3 & 5 \\
\hline Yates, 2001, USA 33 & $+-\cdot+-$ & +- & $? ?+$ & $+?$ & $++?$ & 4 & 4 \\
\hline Zhang, 2006, Japan34 & ++-+ & ++ & $?+?$ & +- & $++?$ & 3 & 4 \\
\hline & Tot & al of unclear a & and fulfill & led vali & idity items & 58 & 84 \\
\hline
\end{tabular}

interventions, ${ }^{24,32,34}$ four exercise interventions, ${ }^{16,18,26,27}$ six balance interventions, ${ }^{21,22,25,31,32}$ one hip protector intervention ${ }^{17}$ and one intervention on fall risk factors $^{23}$ were evaluated. Three interventions explicitly aimed to reduce fear of falling. ${ }^{19,28,34}$ The majority of the interventions aimed to reduce falls and were communitybased. Most trials included ambulatory men and women aged 60 or over. Duration of the interventions varied from one home visit ${ }^{23}$ to exercising at least 1 hour per week for 1 year. ${ }^{16,18,26,27}$ Most trials assessed fear of falling with the Falls Efficacy Scale (FES), ${ }^{1}$ the Modified Falls Efficacy Scale (MFES) ${ }^{40}$ or an adapted version of the FES; about one-third of the trials applied an one-item fear-of-falling measure. ${ }^{16,17,20,26,31,38}$ In seven trials, sample sizes were small, with 50 or fewer participants per group. $20-22,25,31,33,34$

A statistically significant reduction in fear of falling in favour of the intervention group was determined in 12 trials (Table 3). ${ }^{17-19,23,24,27-30,32-34}$ Except for one, ${ }^{16}$ all trials of higher methodological quality reported a statistically significant reduction of fear of falling. ${ }^{17-19,24,27-30,32-34}$ The effective interventions were fall-related multifactorial 
Table 3 General characteristics and outcomes on fear of falling of included trials

\begin{tabular}{llll} 
Study & $\begin{array}{l}\text { N randomised/ } \\
\text { followed-up* }\end{array}$ & Participants \\
\hline Trials of higher methodological quality & & & \\
\hline Barnett, & IG & $83 / 67$ & Aged $\geq 65 ;$ \\
2003, & CG & $80 / 70$ & at risk of falling \\
Australia $^{16}$ & & &
\end{tabular}

$\begin{array}{llll}\text { Cameron, } & \text { IG } & 69 / 61 & \text { Women aged } \geq 75 ; \\ 2000, & \text { CG } & 75 / 70 & \text { at risk of } \\ \text { Australia }^{17} & & & \text { hip fracture }\end{array}$

$\begin{array}{llll}\text { Campbell, } & \text { IG } & 116 / 103 & \text { Women aged } \geq 80 ; \\ \text { 1997, } & \text { CG } & 117 / 109 & \text { ambulatory; } \\ \text { New Zealand } & \text { no physiotherapy }\end{array}$

$\begin{array}{llll}\text { Clemson, } & \text { IG } & 157 / 133 & \text { Aged } \geq 70 ; \\ 2004, & \text { CG } & 153 / 125 & \text { fallen in the past year } \\ \text { Australia }^{19} & & & \text { or concerned about } \\ & & \text { falling }\end{array}$

$\mathrm{Li}$, 2005, USA ${ }^{24,35}$
IG $\quad 125 / 95 / 95$

CG 131/93/93
Aged $\geq 70$;

ambulatory;

inactive 
Fear

Primarily Follow- of falling

Interventions

aimed at ${ }^{\dagger} \quad$ up $^{\ddagger}$ outcome Results $^{\circ}$

IG: Community-based group intervention of a variety of exercises, a home exercise programme and written information for avoiding falls. Weekly sessions of 1 hour for 1 year.

CG: Written information.

IG: Home-based hip protector intervention Hip fracture

$4 \quad$ Fear of and encouragement by phone to wear them midpoint falling for 2 years. One supply visit and 3 home FES and visits in the first 4 months. MFES

CG: Usual care.

IG: Home-based intervention of strength

Falls

12

FES exercises and walking. Four home visits of 1 hour during first 2 months and motivational phone calls to both exercise and walk 3 times per week for 30 minutes for 1 year.

CG: Individual social home visits. Four home visits during first 2 months and motivational phone calls during year of follow-up.

IG: Community-based group intervention of Falls and 14 MFES exercises, medication management, visual fear of falling loss and screening, home and community safety, and action planning. Seven weekly sessions of 2 hours, 1 home visit of 1,5 hours and 1 booster session.

CG: Up to 2 social home visit

IG: Community-based group intervention of tai chi. Six-month programme with 3 sessions Falls

$\begin{array}{ccc}6 & \text { SAFFE } & + \\ 12 & \text { SAFFE } & +\end{array}$
of 1 hour per week.

CG: Community-based group intervention of stretching exercises. Six-month programme with 3 sessions of 1 hour per week. 
Table 3 General characteristics and outcomes on fear of falling of included trials (continued)

\begin{tabular}{llll} 
Study & $\begin{array}{l}\text { N randomised/ } \\
\text { followed-up* }\end{array}$ & Participants \\
\hline Trials of higher methodological quality & & & \\
\hline Robertson, & IG & $121 / 109$ & Aged $\geq 75 ;$ \\
2001, & CG & $119 / 97$ & ambulatory \\
New Zealand27 & & &
\end{tabular}

$\begin{array}{llll}\text { Tennstedt, } & \text { IG } & 216 / 198 / 175 / 170 & \text { Aged } \geq 60 ; \\ 1998, & \text { CG } & 218 / 190 / 168 / 176 & \text { self-reported activity } \\ \text { USA }^{28} & & & \text { restriction due to } \\ & & & \text { fear of falling }\end{array}$

Tinetti,

1994,

USA ${ }^{29}$

IG $\quad 153 / 147$

CG $\quad 148 / 144$
Van Haastregt,

2000,

Netherlands 30
Aged $\geq 70$;

ambulatory;

mobility 1 risk factor for falling

$\begin{array}{ll}\text { IG } & 159 / 129 / 1 \\ \text { CG } & 157 / 123 / 11 \\ & \\ & \\ \text { IG1 } & 64 / 51 / 51 \\ \text { IG2 } & 72 / 60 / 60 \\ \text { CG } & 64 / 54 / 54\end{array}$

Aged $\geq 70$;

mobility 2 falls in past 6

months or impaired

mobility

$\begin{array}{llll}\text { Wolf, } & \text { IG1 } & 64 / 51 / 51 & \text { Aged } \geq 70 ; \\ 1996, & \text { IG2 } & 72 / 60 / 60 & \text { ambulatory } \\ \text { USA }^{32} & \text { CG } & 64 / 54 / 54 & \end{array}$


Fear

Primarily Follow- of falling

Interventions

aimed at ${ }^{\dagger} \quad$ up $^{\ddagger}$ outcome Results $^{\circ}$

IG: Home-based exercise intervention on

Falls

12

MFES

strength and balance, and a walking plan.

Four home visits and a booster visit after 6

months and compliance phone calls to both

exercise 3 times per week for 30 minutes and

walk twice weekly for 1 year.

CG: Usual care.

IG: Community-based cognitive behavioural group intervention on instilling adaptive beliefs on falls, strength exercises, fall risk and safety, safe behaviour and action planning. Four-week programme with twice weekly sessions of 2 hours.

CG: One community-based placebo attention control session of 2 hours on incidence of falls and risk factors for falls.

$\begin{array}{cccc}\begin{array}{c}\text { Fear of } \\ \text { falling and }\end{array} & 1.5 & \begin{array}{c}\text { Adapted } \\ \text { FES, FM }\end{array} & +^{c} \\ \text { activity } & & \text { PCOF } & - \\ \text { restriction } & 6 & \text { FM } & +^{c} \\ & & \text { Adapted } & - \\ & \text { FES, } \\ & \text { PCOF }\end{array}$

12 Adapted +

FES, FM

PCOF

IG: Individual home-based multifactorial intervention addressing risk factors for falling

Falls 12 Adapted + FES by medication adjustment, behavioural instructions or exercise programmes. Mean of 8 home visits in 3 months. CG: Mean of 6 home visits.

IG: Individual home visits to address medical, Falls and 12 and Adapted environmental and behavioural hazards for mobility

impairment CG: Usual care.

\begin{tabular}{|c|c|c|c|c|}
\hline $\begin{array}{l}\text { IG1: Individual computerised balance training. } \\
\text { Weekly sessions of } 45 \text { minutes for } 15 \text { weeks. }\end{array}$ & $\begin{array}{l}\text { Frailty and } \\
\text { falls }\end{array}$ & 4 & $\begin{array}{l}\text { Fear of } \\
\text { falling }\end{array}$ & $+\mid \mathrm{GG} 2$ \\
\hline $\begin{array}{l}\text { IG2: Community-based group intervention of } \\
\text { tai chi. Twice weekly sessions of } 45 \text { minutes }\end{array}$ & & 8 & $\begin{array}{l}\text { Fear of } \\
\text { falling }\end{array}$ & $\begin{array}{l}\text { Not clear } \\
\text { from }\end{array}$ \\
\hline $\begin{array}{l}\text { for } 15 \text { weeks. } \\
\text { CG: Educational group intervention. Weekly } \\
\text { sessions for } 15 \text { weeks. }\end{array}$ & & & & paper \\
\hline
\end{tabular}


Table 3 General characteristics and outcomes on fear of falling of included trials (continued)

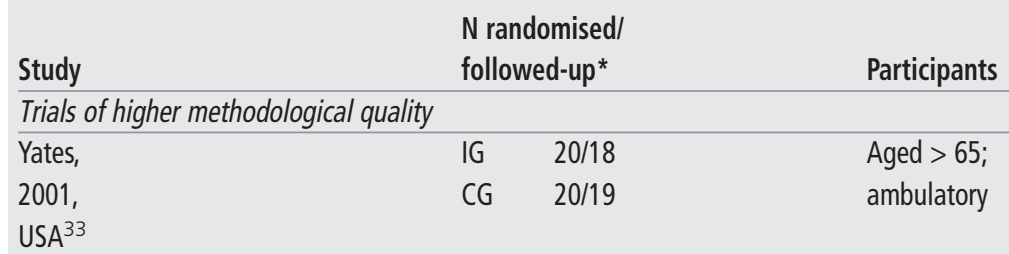

\begin{tabular}{llll}
$\begin{array}{l}\text { Zhang, } \\
2006,\end{array}$ & IG & $\begin{array}{l}25 / 24 / 24 \\
24 / 23 / 23\end{array}$ & $\begin{array}{l}\text { Aged } \geq 60 ; \\
\text { one-leg stance between } \\
5 \text { and 20s; ambulatory }\end{array}$ \\
Japan ${ }^{34}$ & CG & & \\
\hline Trials of lower methodological quality & & & \\
\hline $\begin{array}{l}\text { Gallagher, } \\
1996,\end{array}$ & IG & $50 / 50$ & Aged $\geq 60 ;$ \\
Canada $^{20}$ & CG & $50 / 50$ & fallen in past 3 months
\end{tabular}

$\begin{array}{llll}\text { Hamel, } & \text { IG } & \text { 12/not reported } & \text { Aged } 60 \text { to } 90 ; \\ 2005, & \text { CG } & \text { 8/not reported } & \text { Berg Balance scale }>45 ; \\ \text { Canada }^{21} & & & A B C \text { score } \geq 66 \%\end{array}$

$\begin{array}{llll}\text { Hinman, } & \text { IG1 } & 34 / 28 & \text { Aged 63 to 87; } \\ 2002, & \text { IG2 } & 32 / 30 & \text { ambulatory } \\ \text { USA }^{22} & \text { CG } & 31 / 30 & \end{array}$


Fear

Primarily Follow- of falling

Interventions

aimed at ${ }^{\dagger} \quad$ up $^{\ddagger}$ outcome $\mathrm{R}^{\S}$ Results $^{\circ}$

IG: Multifactorial fall risk reduction

Fall risk

$2.5 \quad$ Adapted

factors

FES

nutrition and environmental hazards.

Ten-week programme.

CG: Usual care (delayed intervention).

IG: Community-based group intervention of

Physical

2

FES

simplified tai chi. Eight-week programme of

functioning

daily sessions of 1 hour.

CG: Usual care.

and fear of

falling

IG: Individual fall risk assessment,

Falls

6

Fear of

counselling, and educational package. Three

falling and

home visits within 2 weeks of about 45

a falls

minutes.

self-efficacy

CG: Usual care.

measure

IG: Group intervention of awareness of physical sensations accompanying mental

Balance

Not

$A B C$

images during static balance and daily

reported

training at home. Weekly group sessions for

6 weeks.

CG: Usual care.

IG1: Individual computerised balance

Balance

1

MFES

training. Four-week programme with three

sessions per week of 20 minutes.

IG2: Home-based intervention of illustrated balance exercises and activities. Four-week programme with three sessions per week of 20 minutes.

CG: Usual care. 
Table 3 General characteristics and outcomes on fear of falling of included trials (continued)

\begin{tabular}{|c|c|c|}
\hline Study & $\begin{array}{l}N \text { randomised/ } \\
\text { followed-up* }\end{array}$ & Participants \\
\hline \multicolumn{3}{|c|}{ Trials of lower methodological quality } \\
\hline Huang, & $60 / 55$ & Aged $\geq 65$ \\
\hline $\begin{array}{l}\text { 2004, } \\
\text { Taiwan²3 }\end{array}$ & $60 / 58$ & \\
\hline
\end{tabular}

Nitz,

IG $\quad 37 / 24 / 19$

Aged $>60$;

2004,

CG $36 / 21 / 13$

fallen in the past year

Australia 25

$\begin{array}{llll}\text { Reinsch, } & \text { IG1 } & 57 / 37 & \text { Aged }>60 \\ 1992, & \text { IG2 } & 51 / 32 & \\ \text { USA }^{26} & \text { IG3 } & 72 / 50 & \\ & \text { CG } & 50 / 23 & \end{array}$

Wolf, IG 47/37/37 $\quad$ Aged $\geq 75$;

2001, CG $\quad 47 / 40 / 40$ impaired balance

Netherlands ${ }^{31}$ during functional activities

${ }^{*} \mathrm{IG}=$ intervention group; $\mathrm{CG}=$ control group

† Outcome that the authors primarily aimed to improve by conducting the intervention.

‡ Follow-up measurement in months after randomisation. "Midpoint" refers to a measurement halfway through the intervention rather than after the intervention.

${ }^{\circ}$ Results on fear of falling measure. $+=$ significant difference in favour of intervention group. $+^{c}=$ significant difference in favour of compliers with the intervention group. - $=$ no significant difference between groups. Significant difference if $p<.05$. 
Fear

Primarily Follow- of falling

Interventions

aimed at ${ }^{\dagger} \quad$ up $^{\ddagger}$ outcome ${ }^{\S}$ Results $^{\circ}$

IG: Home-based intervention of addressing

Falls

4

FES

$+$

individual fall risk factors. One home visit to

identify risk factors and a home visit to

implement intervention after 2 months.

CG: Standardised fall prevention brochure.

IG: Clinic-based balance intervention

Falls $\quad 3$ and 6

FES

comprising individual exercises, small-group

activities and written information on reducing

fall risk. Ten weekly sessions of 1 hour.

CG: Small-group control exercise intervention

and written information on reducing fall risk.

Ten weekly sessions of about 1 hour.

IG1: Group intervention exercises to improve

Falls

$12 \quad$ Fear of

strength and balance. One-year programme

falling

with 3 sessions of 1 hour per week.

IG2: Cognitive behavioural group intervention

on health, safety, relaxation and reaction

time. Weekly sessions of 1 hour for 1 year.

IG3: IG1 and IG2 combined; relaxation and

safety topics were added to exercise sessions.

Weekly cognitive behavioural and twice weekly

exercise sessions of 1 hour for 1 year.

CG: Discussion on topics not fall-related.

Weekly sessions of 1 hour for 1 year.

IG: Individual intervention with balance

exercises and training in activities of daily

living. Twelve sessions of 30 minutes held 2-3

Balance 1 to 1.5

Fear of

dysfunction and falling

times per week.

CG: Individual oriented activities. Twelve

sessions of 30 minutes held 2-3 times per week.

§ Measured fear-of-falling concept; fear of falling $=$ one-item question assessing one of the concepts of fear of falling; FES = Falls Efficacy Scale; ${ }^{1}$ Adapted FES = adapted version of FES, for instance activities in questions adapted to the population concerned; MFES = Modified Falls Efficacy Scale; ${ }^{40}$ MES = Mobility Efficacy Scale; $; 1$ ABC $=$ Activities-specific Balance Confidence scale; 47 SAFFE $=$ Survey of Activities and Fear of Falling in the Elderly; ${ }^{7}$ PCOF $=$ Perceived Control Over Falling; ${ }^{52} \mathrm{FM}=$ perceived ability to manage risk of falls or actual falls.28 
interventions, ${ }^{19,28-30,33}$ tai chi interventions, ${ }^{24,32,34}$ exercise interventions ${ }^{18,27}$ and a hip protector intervention. ${ }^{17}$ With one exception, ${ }^{32}$ all trials using an one-item fear-of-falling question showed no significant difference between groups. ${ }^{16,20,26,31}$ Three of the trials $17,19,28$ that used more than one fear-of-falling measure reported discrepancies in outcomes on the different measures.

Table 4 presents reported process characteristics of the interventions. The observed total number of fulfilled items ranged from 0 to 6 , with a median of 3 . Of all seven process characteristic items across the 19 trials (133 items), 39\% (52/133) were fulfilled. On average 12 of the included trials presented clear information on the facilitator conducting the intervention, compliance of participants or dropout during the intervention. Providing information on performance of the intervention according to protocol, feasibility of the conducted intervention or recommendations for improving the intervention were less common; on average three papers provided some of this information.

Table 4 Process characteristics of the interventions*

\begin{tabular}{|c|c|c|c|c|c|c|c|c|}
\hline Study & a & b & c & $d$ & e & $f$ & g & $\begin{array}{c}\text { Total + } \\
\text { per trial }\end{array}$ \\
\hline \multicolumn{9}{|l|}{ Trials of higher methodological quality } \\
\hline Barnett, 2003 , Australia ${ }^{16}$ & + & + & - & - & - & - & - & 2 \\
\hline Cameron, 2000, Australia ${ }^{17}$ & + & + & - & - & - & - & - & 2 \\
\hline Campbell, 1997, New Zealand ${ }^{18}$ & + & + & + & + & - & - & - & 4 \\
\hline Clemson, 2004, Australia 19 & + & + & + & - & - & - & - & 3 \\
\hline $\mathrm{Li}_{1} 2005$, USA $^{24,35}$ & + & + & + & + & - & - & - & 4 \\
\hline Robertson, 2001, New Zealand 27 & + & + & + & + & - & + & + & 6 \\
\hline Tennstedt, 1998, USA $^{28}$ & - & + & + & + & - & + & + & 5 \\
\hline Tinetti, 1994, USA 29 & + & + & + & + & + & - & - & 5 \\
\hline Van Haastregt, 2000, Netherlands 30 & + & + & + & - & + & - & - & 4 \\
\hline Wolf, 1996, USA ${ }^{32}$ & - & + & + & + & - & - & - & 3 \\
\hline Yates, 2001, USA ${ }^{33}$ & - & + & - & - & - & - & - & 1 \\
\hline Zhang, 2006, Japan³ & + & + & + & + & - & - & - & 4 \\
\hline \multicolumn{9}{|l|}{ Trials of lower methodological quality } \\
\hline Gallagher, 1996, Canada20 & + & + & - & - & - & - & - & 2 \\
\hline Hamel, 2005, Canada 21 & - & - & - & - & - & - & - & 0 \\
\hline Hinman, 2002, USA 22 & - & - & - & - & - & + & - & 1 \\
\hline Huang, 2004, Taiwan²3 & - & - & - & - & - & - & - & 0 \\
\hline Nitz, 2004, Australia 25 & + & + & + & + & - & - & - & 4 \\
\hline Reinsch, 1992, USA 26 & - & - & - & - & - & - & - & 0 \\
\hline Wolf, 2001, Netherlands 31 & + & - & - & - & - & - & + & 2 \\
\hline Total of fulfilled items & 12 & 14 & 10 & 8 & 2 & 3 & 3 & 52 \\
\hline
\end{tabular}




\section{Discussion}

Of the 19 randomised controlled trials that evaluated the effectiveness of an intervention on fear of falling in community-living older people, 12 trials were of higher methodological quality according to the predefined standard. This review showed fairly consistent findings in reducing fear of falling in community-living people in trials of higher methodological quality: two home-based exercise interventions, ${ }^{18,27}$ three community-based tai chi group interventions, ${ }^{24,32,34}$ and five fall-related multifactorial programmes ${ }^{19,28-30,33}$ of which three were home-based ${ }^{29,30,33}$ and two community-based in group format. ${ }^{19,28}$ Most interventions were not primarily aimed to reduce fear of falling and their effects on fear of falling should be considered in the context of their primary objectives as well. With one exception, ${ }^{30}$ these exercise, tai chi and fall-related multifactorial interventions also demonstrated improvement in their primary outcome (e.g., number of falls).

Regarding the effectiveness of trials of higher methodological quality, two observations deserve consideration. First, two trials evaluating a comparable home-based exercise intervention showed consistency in effectiveness ${ }^{18,27}$ but a community-based exercise trial did not support these findings. ${ }^{16}$ Variations in these studies, such as applied fear-of-falling measures (scales versus an insensitive-to-change one-item question ${ }^{41}$ ), may explain these differences. Second, in fall-related multifactorial interventions, community-based group interventions of approximately eight sessions and individual interventions of approximately five home visits resulted in a reduction of fear of falling, ${ }^{19,28-30,33}$ although both community-based multifactorial group interventions reported discrepancies in findings across different measures of fear of falling. ${ }^{19,28}$

Any systematic review is potentially susceptible to bias. An attempt was made to minimise publication bias by supplementing the database search with expert consultation. Expert consultation proved particularly valuable, because it provided approximately one third of the included trials. As a result, it is strongly recommended that experts be consulted in the search for relevant papers for a systematic review, particularly if the outcome of interest is unlikely to be the primary outcome and is not well defined using MeSH terms. To improve reliability regarding data extraction and minimise bias, two independent reviewers extracted data using a standardised format. Blinded data extraction was not possible, because the reviewers were experts in the field of research, but evidence for the superiority of blinded data extraction is inconclusive. ${ }^{11}$ To minimise bias due to study design and quality, only randomised controlled trials were included and an overview of validity items was provided, although the predefined standard for higher methodological quality depended on reporting of validity items by authors. Overall, study reports paid little attention to process characteristics of interventions, meaning that, for most trials, it remains unclear whether the intervention was performed according to protocol.

\section{Implications}

Home-based exercise interventions, community-based tai chi and home-based fall-related multifactorial interventions have been shown to be effective. Because home-based and community-based interventions resulted in a reduction of fear of falling in community- 
living older people, before implementation into practice, careful consideration should be given to the most appropriate intervention for each setting.

Several implications for research arise from our review. First, further research on which types of interventions reduce fear of falling is warranted, because the results were based on a small number of trials. Therefore, fear of falling should be assessed in future trials that address relevant factors such as falls, activity restriction and frailty in older people. More specifically, future research should provide clarity on the effectiveness of balance interventions, community-based exercise interventions and community-based multifactorial group interventions on fear of falling in community-living older people. The evaluation of a community-based multifactorial group intervention is in progress. ${ }^{42}$ Second, to benefit from findings of future trials, these trials should have sufficient power and be of higher methodological quality (see list of criteria in Table 1), wellreported (as recommended in the CONSORT statement ${ }^{43}$ ) and comparable to other trials regarding outcome measures. ${ }^{44}$ For the latter, the FES International (FES-I) should be used as a fear-of-falling measure. This 16-item measure assesses the intensity of concern about falling when performing easy and more-demanding physical and social activities. This measure meets several objections to other fear-of-falling measures and has been shown to have acceptable reliability and construct validity across different samples in various countries. ${ }^{41,45}$ In the past decade, different concepts, such as fear of falling and fall-related self-efficacy, have been applied to describe and measure psychological aspects of falling. ${ }^{12}$ Fear of falling and fall-related self-efficacy may not be considered one and the same construct ${ }^{46}$ and an increase in fall-related self-efficacy, assessed as balance confidence, ${ }^{47}$ can act as a mediator to reduce fear of falling. ${ }^{35}$ This underlines the need to distinguish between these outcomes, although there is evidence that the different dimensions of fear of falling, such as perceived risk and consequences of falling, are all strongly correlated with a single underlying dimension of perceived threat of falling, ${ }^{48}$ although if one is interested in fear of falling as an outcome, applying the FES-I would facilitate comparing findings in different samples in various countries. Reporting effect sizes might also improve comparability of findings across trials. Third, process characteristics should be reported, because they are essential for understanding and improving evaluated interventions. ${ }^{49}$ In addition, besides intentionto-treat analyses, subgroup analyses are recommended to provide insight into the effectiveness of the intervention in people with different characteristics (e.g., people with different initial levels of fear of falling). These findings may help target the most appropriate populations for specific interventions or may provide a reason for adapting an intervention for a particular population (e.g., intensifying an intervention for people with higher levels of fear). Lastly, with regard to most interventions that reduced fear of falling, little is known about underlying mechanisms, including for example, the role of cognitive and physical elements, and the effect of adherence and encouragement as potential contributors to success. Insights into these aspects are important for improving interventions and implementation.

Fear of falling might be a protective response to a realistic threat, preventing people from undertaking activities with a high risk of falling and potential injury, although fear of falling resulting in restriction of activities that one could safely perform may lead to unnecessary adverse consequences regarding social, mental and physical health. ${ }^{4-10}$ It 
is therefore important that future fall-related interventions and research promote a realistic and adaptive view on fall risk and teach older people to perform activities safely. The experience of performing activities safely may lead to increased falls selfefficacy and a realistic view of the risk of falling. Although reducing fear of falling is an important goal in itself to improve psychological well-being in older people, benefits for both older people themselves and society in general may increase if this reduction also results in or is accompanied by increased safe behaviour, social participation, and maintenance or renewed uptake of activities of daily living. Thus, in addition to assessing fear of falling and falls, assessing activity in fall-related trials is also important. Such a measure should not only assess physical activity but should also cover a broader domain of activities, such as activities of daily living and social participation. A recent study showed that there is limited evidence about measures' properties in this area of outcome measures suitable for fall-injury-prevention trials. ${ }^{50}$ Therefore, the development of a suitable measure is desired.

To sum up, community-based tai chi, home-based exercise and home-based fallrelated multifactorial interventions have shown to reduce fear of falling in communityliving older people. Further well-conducted research on interventions that reduce or may reduce fear of falling in older people is warranted.

\section{Acknowledgements}

We kindly thank the following experts who provided their assistance in the expert consultation: N Beyer, F Bishop, E Freiberger, K Hauer, K McKee, C Piot-Ziegler, C Todd and $L$ Yardley, from Workpackage 4 of the Prevention of Falls Network Europe, and $R$ Cumming, K Delbaere, L Gillespie, F Li, S Lord and C Robertson. Lesley Gillespie is gratefully acknowledged for her constructive comments on an earlier version of this paper.

This review is part of a study funded by ZonMw - The Netherlands Organisation for Health Research and Development (grant 014-91-052), the Care and Public Health Research Institute, and the Faculty of Health, Medicine and Life Sciences of the Maastricht University. 


\section{References}

1. Tinetti ME, Richman D, Powell L. Falls efficacy as a measure of fear of falling. J Gerontol 1990;45:P239-43.

2. Fletcher PC, Hirdes JP. Restriction in activity associated with fear of falling among community-based seniors using home care services. Age Ageing 2004;33:273-79.

3. Howland J, Lachman ME, Peterson EW, et al. Covariates of fear of falling and associated activity curtailment. Gerontologist 1998;38:549-55.

4. Yardley L, Smith H. A prospective study of the relationship between feared consequences of falling and avoidance of activity in community-living older people. Gerontologist 2002;42:17-23.

5. Friedman SM, Munoz B, West SK, et al. Falls and fear of falling: which comes first? A longitudinal prediction model suggests strategies for primary and secondary prevention. J Am Geriatr Soc 2002;50:1329-35.

6. Howland J, Peterson EW, Levin WC, et al. Fear of falling among the communitydwelling elderly. J Aging Health 1993;5:229-43.

7. Lachman ME, Howland J, Tennstedt $S$, et al. Fear of falling and activity restriction: the survey of activities and fear of falling in the elderly (SAFE). J Gerontol B Psychol Sci Soc Sci 1998;53:P43-50.

8. Arfken $\mathrm{CL}$, Lach HW, Birge SJ, et al. The prevalence and correlates of fear of falling in elderly persons living in the community. Am J Public Health 1994;84:565-70.

9. Cumming RG, Salkeld G, Thomas M, et al. Prospective study of the impact of fear of falling on activities of daily living, SF-36 scores, and nursing home admission. $J$ Gerontol A Biol Sci Med Sci 2000;55:M299-305.

10. Delbaere K, Crombez G, Vanderstraeten G, et al. Fear-related avoidance of activities, falls and physical frailty. A prospective community-based cohort study. Age Ageing 2004;33:368-73.

11. Cochrane Handbook for Systematic Reviews of Interventions 4.2.5. [updated May 2005]; Appendix 5b. Chichester: Wiley, 2005.

12. Jorstad EC, Hauer K, Becker $C$, et al. Measuring the psychological outcomes of falling: a systematic review. J Am Geriatr Soc 2005;53:501-10.

13. Skelton DA, Becker C, Lamb SE, et al. Prevention of Falls Network Europe: a thematic network aimed at introducing good practice in effective falls prevention across Europe. Eur J Ageing 2004;1:89-94.

14. van Tulder MW, Assendelft WJ, Koes BW, et al. Method guidelines for systematic reviews in the Cochrane Collaboration Back Review Group for Spinal Disorders. Spine 1997;22:2323-30.

15. van Tulder MW, Cherkin DC, Berman B, et al. The effectiveness of acupuncture in the management of acute and chronic low back pain. A systematic review within the framework of the Cochrane Collaboration Back Review Group. Spine 1999;24:1113-23.

16. Barnett A, Smith B, Lord SR, et al. Community-based group exercise improves balance and reduces falls in at-risk older people: a randomised controlled trial. Age Ageing 2003;32:407-14.

17. Cameron ID, Stafford B, Cumming RG, et al. Hip protectors improve falls self-efficacy. Age Ageing 2000;29:57-62. 
18. Campbell AJ, Robertson MC, Gardner MM, et al. Randomised controlled trial of a general practice programme of home based exercise to prevent falls in elderly women. BMJ 1997;315:1065-9.

19. Clemson L, Cumming RG, Kendig $H$, et al. The effectiveness of a community-based program for reducing the incidence of falls in the elderly: a randomized trial. J Am Geriatr Soc 2004;52:1487-94.

20. Gallagher EM, Brunt H. Head Over Heels: Impact of a health promotion program to reduce falls in the elderly. Can J Aging 1996;15:84-96.

21. Hamel MF, Lajoie Y. Mental imagery. Effects on static balance and attentional demands of the elderly. Aging Clin Exp Res 2005;17:223-8.

22. Hinman MR. Comparison of two short-term balance training programs for communitydwelling older adults. J Geriatr Phys Ther 2002;25:10-5.

23. Huang TT, Acton GJ. Effectiveness of home visit falls prevention strategy for Taiwanese community-dwelling elders: randomized trial. Public Health Nurs 2004;21:247-56.

24. Li F, Harmer P, Fisher KJ, et al. Tai chi and fall reductions in older adults: a randomized controlled trial. J Gerontol A Biol Sci Med Sci 2005;60:187-94.

25. Nitz JC, Choy NL. The efficacy of a specific balance-strategy training programme for preventing falls among older people: a pilot randomised controlled trial. Age Ageing 2004;33:52-8.

26. Reinsch S, MacRae P, Lachenbruch PA, et al. Attempts to prevent falls and injury: a prospective community study. Gerontologist 1992;32:450-6.

27. Robertson MC, Devlin N, Gardner MM, et al. Effectiveness and economic evaluation of a nurse delivered home exercise programme to prevent falls. 1: Randomised controlled trial. BMJ 2001;322:697-701.

28. Tennstedt S, Howland J, Lachman M, et al. A randomized, controlled trial of a group intervention to reduce fear of falling and associated activity restriction in older adults. $J$ Gerontol B Psychol Sci Soc Sci 1998;53:P384-92.

29. Tinetti ME, Baker DI, McAvay G, et al. A multifactorial intervention to reduce the risk of falling among elderly people living in the community. N Engl J Med 1994;331:821-7.

30. van Haastregt JC, Diederiks JP, van Rossum E, et al. Effects of a programme of multifactorial home visits on falls and mobility impairments in elderly people at risk: randomised controlled trial. BMJ 2000;321:994-8.

31. Wolf $B$, Feys $H$, De W, et al. Effect of a physical therapeutic intervention for balance problems in the elderly: a single-blind, randomized, controlled multicentre trial. Clin Rehabil 2001;15:624-36.

32. Wolf SL, Barnhart HX, Kutner NG, et al. Reducing frailty and falls in older persons: an investigation of Tai Chi and computerized balance training. Atlanta FICSIT Group. Frailty and Injuries: Cooperative Studies of Intervention Techniques. J Am Geriatr Soc 1996;44:489-97.

33. Yates SM, Dunnagan TA. Evaluating the effectiveness of a home-based fall risk reduction program for rural community-dwelling older adults. J Gerontol A Biol Sci Med Sci 2001;56:M226-30.

34. Zhang JG, Ishikawa-Takata K, Yamazaki H, et al. The effects of Tai Chi Chuan on physiological function and fear of falling in the less robust elderly: an intervention study for preventing falls. Arch Gerontol Geriatr 2006;42:107-16. 
35. Li F, Fisher KJ, Harmer P, et al. Falls self-efficacy as a mediator of fear of falling in an exercise intervention for older adults. J Gerontol B Psychol Sci Soc Sci 2005;60:P34-40.

36. Li F, Harmer P, Fisher KJ, et al. Tai Chi: improving functional balance and predicting subsequent falls in older persons. Med Sci Sports Exerc 2004;36:2046-52.

37. Li F, Harmer P, McAuley E, et al. An evaluation of the effects of Tai Chi exercise on physical function among older persons: a randomized contolled trial. Ann Behav Med 2001;23:139-46

38. Wolf SL, Barnhart HX, Ellison GL, et al. The effect of Tai Chi Quan and computerized balance training on postural stability in older subjects. Atlanta FICSIT Group. Frailty and Injuries: Cooperative Studies on Intervention Techniques. Phys Ther 1997;77:371-381; discussion 382-74.

39. Wolf SL, Barnhart HX, Kutner NG, et al. Selected as the best paper in the 1990s: Reducing frailty and falls in older persons: an investigation of tai chi and computerized balance training. J Am Geriatr Soc 2003;51:1794-803.

40. Hill KD, Schwarz JA, Kalogeropoulos AJ, et al. Fear of falling revisited. Arch Phys Med Rehabil 1996;77:1025-9.

41. Yardley L, Beyer N, Hauer K, et al. Development and initial validation of the Falls Efficacy Scale-International (FES-I). Age Ageing 2005;34:614-19.

42. Zijlstra GA, van Haastregt JC, van Eijk JT, et al. Evaluating an intervention to reduce fear of falling and associated activity restriction in elderly persons: design of a randomised controlled trial [ISRCTN43792817]. BMC Public Health 2005;5:26.

43. Moher D, Schulz KF, Altman DG. The CONSORT statement: revised recommendations for improving the quality of reports of parallel-group randomised trials. Lancet 2001;357:1191-4.

44. Lamb SE, Jorstad-Stein EC, Hauer K, et al. Development of a common outcome data set for fall injury prevention trials: the prevention of falls network europe consensus. J Am Geriatr Soc 2005;53:1618-22.

45. Kempen Gl, Todd CJ, van Haastregt JC, et al. Cross-cultural validation of the Falls Efficacy Scale International (FES-I) in older people: results from Germany, The Netherlands and the UK were satisfactory. Disabil Rehabil 2007;29(2):155-62.

46. McKee KJ, Orbell S, Austin CA, et al. Fear of falling, falls efficacy, and health outcomes in older people following hip fracture. Disabil Rehabil 2002;24:327-33.

47. Powell LE, Myers AM. The Activities-specific Balance Confidence (ABC) Scale. J Gerontol A Biol Sci Med Sci 1995;50a:M28-34.

48. Yardley L, Donovan-Hall M, Francis K, et al. Attitudes and beliefs that predict older people's intention to undertake strength and balance training. J Gerontol B Psychol Sci Soc Sci 2007;62(2):119-25.

49. Oakley A, Strange $V$, Bonell $C$, et al. Process evaluation in randomised controlled trials of complex interventions. BMJ 2006;332:413-6.

50. Jorstad-Stein EC, Hauer K, Becker C, et al. Suitability of physical activity questionnaires for older adults in fall-prevention trials: a systematic review. J Aging Phys Act 2005;13:461-81.

51. Lusardi MM, Smith EV, Jr. Development of a scale to assess concern about falling and applications to treatment programs. J Outcome Meas 1997;1:34-55.

52. Lachman ME, Weaver SL, Bandura M, et al. Improving memory and control beliefs through cognitive restructuring and self-generated strategies. J Gerontol 1992;47:P293-9. 



\section{CHAPTER 4}

\section{Reducing fear of falling and avoidance of activity in elderly persons: the development of a Dutch version of an American intervention}




\section{Abstract}

Objective The present study reports on the development of a Dutch version of an American intervention for community-residing older persons in The Netherlands. Adaptation of this cognitive behavioural group intervention, to reduce fear of falling and avoidance of activity in older persons, was required before evaluation in a different setting.

Methods The process of adaptation consisted of defining the target population, translating the original intervention manual literally, consulting Dutch experts and a developer of the original intervention, selecting qualified facilitators and conducting a pilot study of the adapted manual.

Results Adaptations were incorporated to improve the content, feasibility and didactic materials. The main adaptations were scheduling more time for some activities, changing session frequency from twice to once a week, adding a booster session after 6 months and adding more transparencies.

Conclusions A critical assessment of the appropriateness and feasibility of the original intervention provided important information to facilitate replication in the Dutch setting. Applying a systematic approach is recommended in the process of adapting an original intervention for use in a different setting. To facilitate replication, in general, more detailed information should be provided about interventions. 


\section{Introduction}

Fear of falling is common in older persons. It is reported both by older people who experienced a fall and by those who have no previous fall experience. ${ }^{1-4}$ In studies conducted in the US, UK and The Netherlands, about 20 to $60 \%$ of the older people living in the community reported at least some fear of falling. ${ }^{2}$ 5-11 In two studies an even higher percentage of older people who reported fear of falling was observed. ${ }^{12}{ }^{13}$ Population characteristics, like age, fall history and frailty, and measures used to assess fear of falling differ greatly among studies and may cause the variability observed in prevalence of fear of falling. ${ }^{7415}$ Besides all this, it is likely that prevalence of fear of falling is underreported due to failing to include the most fearful elderly in studies and the fear of stigmatisation. ${ }^{2} 16$

In the past two decades several cross-sectional and a few prospective studies have identified multiple factors associated with fear of falling, including advanced age, 56 female gender, 568 fall history, ${ }^{16817}$ depressed mood, ${ }^{6}{ }^{17-20}$ perceived poor health, ${ }^{15}$ decreased quality of life, ${ }^{621}$ decreased mobility ${ }^{6} 1721$ and institutionalisation. ${ }^{21}$ When fear of falling results in restricting one's activities ${ }^{1347-9} 182223$ and reducing one's physical fitness, it increases the risk for future falls, ${ }^{7}$ mortality, morbidity, reduced functioning and premature nursing home admissions. ${ }^{24}$

With respect to the abovementioned prevalence and potential impact of fear of falling on older people's life, it is obvious that fear of falling may not be ignored. According to Cumming and colleagues it might be just as serious a health problem as falls themselves. ${ }^{21}$ However, in contrast to the many interventions developed to prevent falls, only a few interventions have been developed and evaluated specifically to reduce fear of falling in older people living in the community. ${ }^{25-27}$ One of these interventions, an American cognitive behavioural group intervention, aims at reducing both fear of falling and associated activity restriction in older persons. This intervention, called 'A Matter of Balance' (AMB), was developed and evaluated by Tennstedt and colleagues in Boston, US. ${ }^{26} 28$ Based on the reported effectiveness of this intervention, we decided to perform a randomised controlled trial to assess the effectiveness of AMB in The Netherlands (the design of this trial is described elsewhere ${ }^{29}$ ). For this reason, adaptation of $A M B$ for the Dutch setting was necessary.

When adapting an intervention one should aim at facilitating successful implementation in the new setting on the one hand and preventing unnecessary deviations from the intervention manual on the other hand. Since adaptations may unintentionally result in a loss of effectiveness, it is imperative to replicate interventions as accurately as possible. This implies that researchers performing replication studies need to consider several activities in the replication process. These activities may comprise: examining the original intervention carefully to determine appropriate eligibility criteria for participants, examining the need to update the intervention with new knowledge, determining the feasibility and appropriateness of the intervention in the new setting, and examining potential consequences of adaptations made to the intervention. In the present study to develop a Dutch version of AMB (AMB-NL) for older people in The Netherlands, a systematic approach was applied to facilitate successful implementation and to minimise the risk of reducing the effectiveness of AMB. The aim of this paper is to describe the development process of AMB-NL for community-residing older persons in The Netherlands. 


\section{A Matter of Balance}

AMB is a cognitive behavioural group intervention developed to reduce fear of falling and promote physical, social and functional activity in older persons living in the community. ${ }^{26}$ To attain a reduction in fear of falling, the intervention aims to increase self-efficacy beliefs with regard to falling as well as the sense of control over falling. Four strategies are used to accomplish these aims: (1) restructuring misconceptions to promote a view of fall risk and fear of falling as controllable; (2) setting realistic goals for increasing activity; (3) changing the environment to reduce fall risk; and (4) promoting physical exercise to increase strength and balance. Through use of these strategies, the four main sources to influence one's self-efficacy beliefs, including performance attainment (skills mastery), vicarious experience (modelling), verbal persuasion and physiological states, ${ }^{30}$ are incorporated in AMB.

$A M B$ was developed for older persons living in the community who experience fear of falling and restrict their activity due to this fear of falling. Tennstedt and colleagues included older persons of at least 60 years of age living in senior housing sites who were not suffering from any major physical or health condition, were able to communicate in English and reported activity restriction due to fear of falling. ${ }^{26}$ Participants were recruited through self-response and by provider referrals. Home visits were conducted to screen for eligibility and to obtain informed consent to participate. According to Tennstedt and colleagues, there are several important qualifications for a facilitator of $A M B,{ }^{28}$ including a thorough understanding of the contents and objectives of $A M B$, knowledge of group processes and experience in managing groups, and familiarity with the special needs of older people. In total, eight group sessions constitute the intervention. Each session lasts 2 hours and sessions are held twice a week for a period of 4 weeks. On average, 10 people participate in each group. The contents of each intervention session are described in detail in a facilitator's manual. ${ }^{28}$ The main contents of $A M B$, presented in Appendix A, are addressed in a similar approach: (1) introduction; (2) participant's point of view; (3) positive and negative aspects concerning the topic; (4) the association with falls or fear of falling; and (5) implementation in the participant's daily life. To ensure cognitive restructuring in participants and to stimulate active participation, a variety of techniques and materials are used, including lectures, videos, group discussions, mutual problem-solving, role-plays, physical exercises for strength, flexibility and balance, and skill development. Behavioural contracts and goal setting are used to individualise the intervention. ${ }^{28}$ In all sessions, transparencies and flipcharts are employed for visualisation of information and participants are provided printed materials for completion, either during the session or at home. Participants also receive an elastic band for performing physical exercises during the sessions and at home.

To study the effectiveness of $A M B$, a randomised controlled trial was conducted between October 1994 and July 1996 in 20 senior housing sites in Boston. ${ }^{26}$ The intention-to-treat analysis showed statistically significant improvements in mobility and intended activity directly after the intervention. At 12 months, significant improvement in falls efficacy, mobility and social function was observed in those participants attending five or more sessions. Based on the results of the trial and programmatic experience, Tennstedt and colleagues made several recommendations, ${ }^{26}$ including offering the 
intervention in the home to facilitate participation by those at greater risk of falls and fear of falling, and adding a booster session at 6 months to overcome the observed decay in intervention effect. This booster session was developed and added to the manual by Tennstedt and colleagues. ${ }^{28}$ It includes discussing the benefits of a positive attitude towards fall prevention, responding to a fall, sharing fall experiences and personal actions to reduce recurrence of falling, and discussing perceived changes in behaviour and thoughts as a result of the intervention.

\section{Methods}

In the process of developing $\mathrm{AMB}-\mathrm{NL}$, the original intervention manual and the trial conducted by Tennstedt and colleagues ${ }^{26} 28$ were taken as points of departure, and a systematic approach was developed to obtain an accurate replication of the intervention for the Dutch setting. This approach comprised several activities that led to various preliminary versions before a final version of AMB-NL was formulated (Figure 1). First, $A M B$ was examined to gain insight into the objectives and contents on the one hand and eligibility criteria for participants and qualifications for facilitators on the other hand. Second, the intervention manual was literally translated to obtain an accurate replica of $A M B$ in the Dutch language (the first version of $A M B-N L$ ). Next, this version was reviewed by Dutch experts in gerontology and geriatrics who then were consulted to decide on: (1) the eligibility criteria for participants; (2) the qualifications for the facilitators; (3) the need for updating the intervention manual with new knowledge in the research field; and (4) the appropriateness of the AMB content and activities for the Dutch population. Three authors $(\mathrm{GZ}, J v H, G K)$ evaluated the experts' comments and all authors (except ST) reviewed this first version of AMB-NL while paying attention to feasibility and future implementation of the intervention in the Dutch setting. Adaptations were made to the intervention manual and a second version of AMB-NL was formulated. Next, a pilot study was conducted to evaluate the second version of AMB-NL. Since this study was conducted to improve the manual and not to conduct an effectiveness evaluation; only one group of participants was included. This pilot study aimed to provide insight into: (1) the feasibility of the manual for the facilitators; (2) the appropriateness of the contents of the manual for the participants; and (3) the appropriateness of the programme materials and didactic techniques for the participants. Every session of the intervention was observed by an author (GZ, JvH, GK) and at least one non-participating facilitator. During the pilot study, data was gathered by written reports, questionnaires, discussions, and videotaping participants and facilitators. Directly after the intervention, an evaluation group meeting was held with all facilitators and interviews were conducted with pairs of participants. Following data analysis, adaptations were made to the intervention manual (third version of AMB-NL). Lastly, during several consultations with one of the developers of AMB (author ST), adaptations and their potential consequences in the context of the US experience with $A M B$ were discussed. As a result, adaptations were made resulting in the final version of AMB-NL. The study was approved by the local medical ethics committee. 


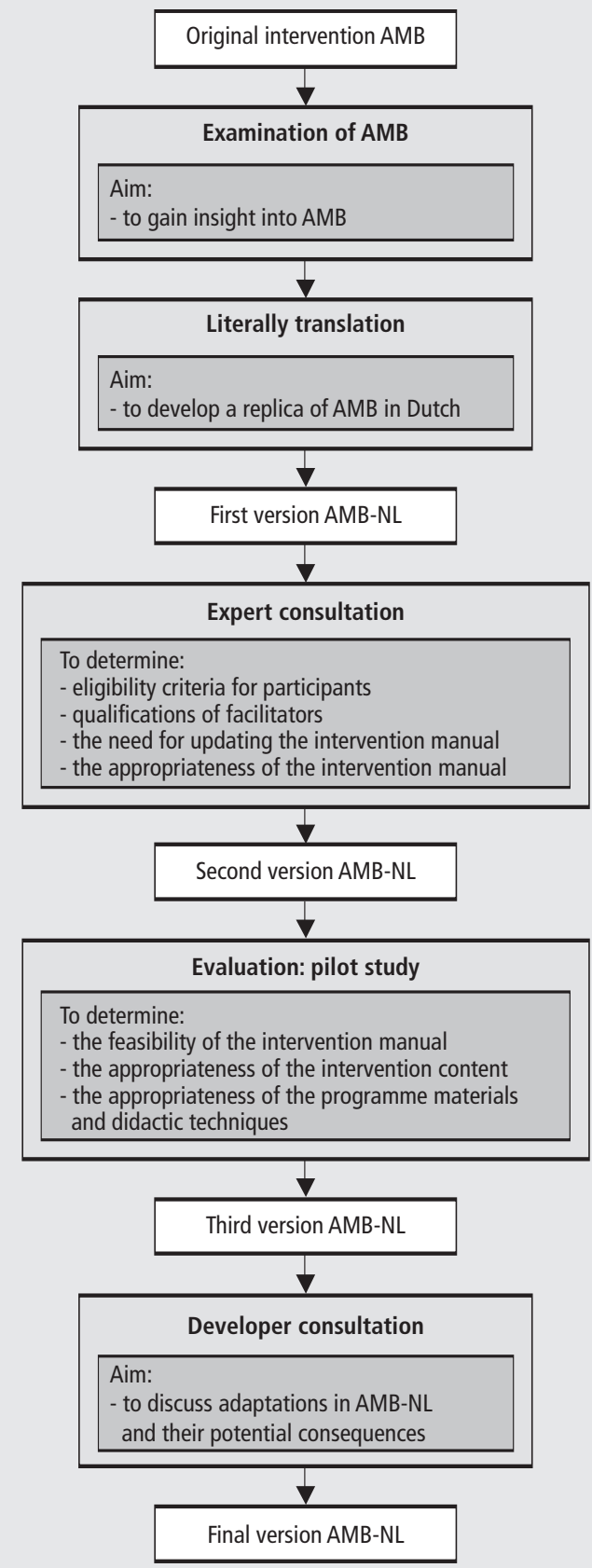

Figure 1 The development process of AMB-NL 


\section{Results}

\section{Examination of $A M B$, literally translation and expert consultation}

After examining $\mathrm{AMB}$ and the population of the American trial, it was decided to use similar inclusion criteria to define the Dutch target population, i.e. older persons reporting at least some fear of falling and associated restriction of activities. Table 1 presents similarities and differences in the target population in AMB and AMB-NL. There were several deviations from the American study. First, the American intervention was conducted in senior housing buildings in order to facilitate attendance by those who had restricted activity. Considering future implementation issues, we preferred to approach all older people living independently in the community. To facilitate attendance, as in the American trial, the intervention was planned to be conducted in the centre of the neighbourhoods and transportation was offered to those experiencing mobility problems. Second, to increase efficiency in selection of the population, the sample was restricted to persons aged 70 or over as prevalence of fear of falling increases with age. ${ }^{5}{ }^{6} \mathrm{Next}$, a brief self-administered questionnaire was developed to screen potential participants rather than a home visit as in the American trial. ${ }^{26}$ This questionnaire efficiently screened a large sample of older persons for the trial evaluating the effectiveness of AMB-NL. Sample size calculations for this trial showed that a minimum sample of 3,560 community-living older persons needed to be screened for eligibility to enrol 284 participants. Lastly, people confined to bed, restricted by permanent use of wheelchair or waiting for nursing home admission were excluded due to expected difficulties in attending and participating in sessions.

In the examination of $A M B$, the qualifications for facilitators as stated by Tennstedt and colleagues ${ }^{28}$ were critically assessed as well. Six nurses working at home care agencies, providing home care to older people living independently in the community and qualified in the field of geriatrics were selected as facilitators for the pilot study and randomised controlled trial in The Netherlands. All nurses had higher vocational level. Before the pilot study, a training session for the facilitators was held. Main topics

Table 1 Comparing the target population of $\mathrm{AMB}^{26} 28$ and AMB-NL

\begin{tabular}{|c|c|}
\hline Target population of AMB & Target population of AMB-NL \\
\hline \multicolumn{2}{|l|}{ Inclusion criteria } \\
\hline reporting fear of falling & reporting fear of falling \\
\hline reporting restriction of activity & reporting avoidance of activity \\
\hline independently living in senior housing sites & independently living in the community* \\
\hline \multicolumn{2}{|l|}{ Exclusion criteria } \\
\hline $\begin{array}{l}\text { suffering from major physical or health condition } \\
\text { unable to communicate in English }\end{array}$ & $\begin{array}{l}\text { confined to bed }^{\dagger} \\
\text { restricted by permanent use of wheelchair } \\
\text { on waiting list for nursing home admission }\end{array}$ \\
\hline
\end{tabular}


addressed were the contents and objectives of AMB-NL, principles of group processes and managing a group. Physical exercises were practiced and performance was evaluated by a physical therapist. A psychologist informed the facilitators about the theoretical background of fear and cognitive behavioural interventions.

The next activity in the systematic approach of developing AMB-NL was literally translation: the AMB manual and all materials were literally translated into Dutch by native speakers (authors $\mathrm{GZ}, \mathrm{JvH}$ ). This included development of Dutch subtitles for the intervention videos as well. Three authors $(G Z, J v H, G K)$ reviewed the intervention manual to improve readability and clarity, and changes were incorporated into the first version of AMB-NL.

Then, several independent experts in gerontology and geriatrics (three nurses, two physical therapists, a geriatrician and a behavioural scientist) reviewed and commented on this first version of AMB-NL. Table 2 shows the comments provided and the incorporated adaptations. The experts agreed with the eligibility criteria for the participants and the qualifications for the facilitators; moreover, the manual did not

Table 2 Comments on AMB-NL following expert consultation and incorporated adaptations in the second version of $A M B-N L$

Comments

\section{Contents}

No update with new insights required

No crucial information lacked ${ }^{*}$

No information was redundant ${ }^{*}$

Activities are relevant and appropriate

Add activities to practice activities of daily life

(e.g., how to walk safely with a cane) ${ }^{*}$

Repeat main topics in the midcourse evaluation

Show assistive devices which improve safety ${ }^{*}$
Incorporated adaptations

Not applicable

Not applicable

Not applicable

Not applicable

None, due to expected limitations in feasibility, e.g., limited time, variety in participants' characteristics

Midcourse evaluation was extended to review all main topics addressed at that time Showing several assistive devices, like a cane, elastic shoelaces and grab bars, was included in session 7

Discuss no new information in the booster session ${ }^{\dagger}$ All new activities were incorporated in previous sessions. The booster session comprised reviewing the main topics and incorporating them in daily life

Provide information on fear of falling relevant to Dutch elderly ${ }^{\dagger \neq}$

Replace addresses for local addresses ${ }^{\dagger \neq}$

Substitute American examples, names or situations $^{\dagger \neq}$
Fact sheets were completed with prevalence rates for falls and fear of falling in The Netherlands Addresses for assistive devices, emergency-calling systems, exercise groups, welfare and safety recommendations in the home environment were replaced by local addresses American examples, names or situations were substituted by one's familiar to Dutch older persons 
Table 2 Comments on AMB-NL following expert consultation and incorporated adaptations in the second version of $\mathrm{AMB}-\mathrm{NL}$ (continued)

\begin{tabular}{|c|c|}
\hline Comments & Incorporated adaptations \\
\hline \multicolumn{2}{|l|}{ Feasibility } \\
\hline Insufficient time scheduled for some activities ${ }^{*}{ }^{\dagger}$ & $\begin{array}{l}\text { A few activities were left out or altered into an } \\
\text { activity with similar goals but taking less time }\end{array}$ \\
\hline $\begin{array}{l}\text { Insufficient time scheduled for challenging fear } \\
\text { of falling }{ }^{+}\end{array}$ & $\begin{array}{l}\text { A handout about challenging fear of falling was } \\
\text { included in three sessions as an additional activity }\end{array}$ \\
\hline Insufficient time scheduled for some sessions ${ }^{*}{ }^{\dagger}$ & $\begin{array}{l}\text { The number of options in optional activities was } \\
\text { reduced or optional activities were omitted }\end{array}$ \\
\hline Expect practical limitations at intervention sites ${ }^{\ddagger}$ & $\begin{array}{l}\text { One activity was modified into an activity with } \\
\text { similar goals }\end{array}$ \\
\hline Provide safety with regard to physical exercises ${ }^{*}+$ & $\begin{array}{l}\text { Facilitators were trained in physical exercises by a } \\
\text { physical therapist. In the intervention manual extra } \\
\text { attention was paid to safely perform physical } \\
\text { exercises }\end{array}$ \\
\hline \multicolumn{2}{|l|}{ Didactic materials } \\
\hline Clarification of translated English phrases $^{*}{ }^{\dagger}$ & $\begin{array}{l}\text { English phrases with no similar Dutch phrases were } \\
\text { explained extensively in the intervention manual }\end{array}$ \\
\hline Phrases on printed materials may be too difficult ${ }^{*}+$ & $\begin{array}{l}\text { Sentences and words on printed materials were } \\
\text { simplified }\end{array}$ \\
\hline Clarify didactic materials and methods ${ }^{*}$ & $\begin{array}{l}\text { Manageability of didactic materials was improved } \\
\text { e.g., by enlarging prints, adding colours and } \\
\text { providing extra instructions in the intervention } \\
\text { protocol }\end{array}$ \\
\hline Visualise more didactic materials ${ }^{*}$ & $\begin{array}{l}\text { Extra transparencies were added to stimulate the } \\
\text { participant's contribution in the activities, as well as } \\
\text { to facilitate the learning process }\end{array}$ \\
\hline
\end{tabular}

require an update with new knowledge according to the experts. However, the experts did provide several comments on the intervention manual and three authors (GZ, JvH, GK) evaluated these comments. Comments on AMB-NL resulting from this process concerned the intervention content, materials, feasibility and implementation in the Dutch setting. All authors (except ST) reviewed AMB-NL as well. Those comments resulting in adaptations included: (a) exclusion of new information in the booster session; (b) more time for some activities or sessions; (c) clarification of Dutch phrases to understand some English phrases completely; (d) simplification and visualisation of materials; and (e) substitution of Dutch examples, addresses, figures with respect to falls, fear of falling, etc. The adaptations were incorporated in the second version of AMB-NL. 


\section{Evaluation and developer consultation}

The next activity was evaluation of the second version of AMB-NL in a pilot study; in the fall of 2002 this study was conducted in the southeast of The Netherlands. Seventeen older people received the screening questionnaire and 11 persons met the inclusion criteria and signed informed consent. Due to pre-existent medical reasons, one of the participants dropped out after the second session. All others completed the eight sessions and the booster session. All trained facilitators participated in the intervention; each facilitator conducted at least one session and observed at least one other session. Comments and adaptations as a result of this pilot study are summarised in Table 3 .

Table 3 Comments following the pilot study and incorporated adaptations in the third concept of AMB-NL

Comments
Feasibility
Insufficient time scheduled for some activities
Insufficient time scheduled for some sessions

Preparing the slideshow takes up too much time
Managing all activities in the first session is difficult
Incorporated adaptations

Sequence of several activities within sessions was altered to improve efficiency Some activities were combined and optional activities were omitted

The slides were printed on transparencies Two facilitators were scheduled to conduct the first session

\section{Didactic materials}

Simplification of phrases derived from $\mathrm{AMB}^{\dagger}$

Some Dutch phrases did not completely correspond with phrases in AMB due to difficulties in translation, facilitators were instructed to explain these phrases to the participants sufficiently

Reduce complexity and improve readability of Sentences and words were simplified and the printed materials and transparencies* ${ }^{\dagger}$ amount of text was reduced Improve accessibility of printed materials* + Printed materials received page numbers and those that returned frequently were printed on coloured paper

Improve consistency among printed materials* Lay-out was improved and comparable printed materials (like personal action planners) were made equal

Apply more transparencies* ${ }^{+}$ For each session a set of transparencies was provided with all printed materials and main activities

* Comment provided by participants.

${ }^{\dagger}$ Comment provided by facilitators and observers of the intervention. 
With regard to the first aim of the pilot study, determining the feasibility of the manual, the facilitators were generally comfortable with the content and the structure of the sessions. However, the time allotted for some of the activities was insufficient. During the evaluation group meeting, facilitators indicated that combining some activities would improve feasibility and efficiency. In addition, recommendations were made with respect to efficiently using the time available and simplifying the manual, e.g., by leaving out optional activities and organising activities more strictly. No major problems were observed or reported regarding managing group processes. However, use of two facilitators to conduct the first session was preferred; firstly, to facilitate managing all activities, and secondly, to introduce the second facilitator as a potential stand-in for the first facilitator during the following sessions. The facilitators also suggested improvements for the printed materials and the use of more transparencies for the intervention content.

With regard to the second aim of the pilot study, determining the appropriateness of the manual content for the participants, both facilitators and observers considered the content appropriate. Participants also rated most topics addressed in the various sessions appropriate and the provided examples were valued as appropriate and useful. During the evaluation interview, participants reported an increase in confidence, independence, self-awareness, increased social and physical activity, and assertiveness. In addition, they reported having implemented adjustments in the home environment and having incorporated adaptations to reduce risk-taking behaviour in daily life.

The final aim of the pilot study was to determine the appropriateness of the materials and didactic techniques applied in the intervention. Participants had recommendations only about the intervention materials. Most participants appreciated the videos with subtitling in Dutch. Participants indicated that printed materials should be improved with regard to complexity, readability, accessibility and consistency. Participants valued the transparencies and recommended using them more frequently. Like the printed materials, simplifying the text of the transparencies was suggested. Provision of the elastic band utilised in the physical exercises was highly appreciated.

The final activity of the systematic approach for developing a Dutch version of AMB included consultation with one of the developers of AMB (ST). Several consultations were arranged to discuss the adaptations to $\mathrm{AMB}$ and their potential consequences. As a result, two final adaptations were made. First, the booster session was added 6 months after the eighth session to prevent decay of intervention effects. Second, session frequency was changed from twice a week to once a week for practical reasons (i.e. availability of intervention location and facilitators) and also to facilitate participant's scheduling of the intervention in their daily life. Attending the sessions and working on the home assignments require time. A decrease in attendance for these older persons was expected when conducting the sessions twice a week. Finally, the content of all sessions was reviewed and the final version of AMB-NL was formulated. 


\section{Discussion}

Particular challenges arise when a replication of an intervention is developed in another country with a different culture. This paper describes the process of developing a Dutch version of an American intervention to reduce fear of falling and avoidance of activity in older persons. The results indicate that it is very important to critically and systematically assess the appropriateness and feasibility of an intervention before implementing it in a different setting. By applying a systematic approach as in the present study, several aspects of the intervention were identified that needed improvement in order to conduct the intervention in the Dutch setting. As a result, a considerable number of actions were undertaken to improve the content, feasibility and didactic materials in AMB-NL.

In the present study, the intervention manual and all additional materials were translated into Dutch and the videos were subtitled. Although the participants appreciated the videos, it is our opinion that replacing them with videos produced in The Netherlands would improve the effect of the videos. We assume that videos with Dutch persons and situations comparable to the Dutch setting will facilitate the participant's identification with the persons in the video and should enhance the effect of modelling on desired behaviour change. It is our opinion as well that the intervention manual could have been further improved if an additional pilot study was conducted to test the final version of AMB-NL. In addition, inclusion of two intervention groups in a pilot study would provide more information to assess if adaptations were indeed necessary or if recommendations for adaptation were specific to this particular group of participants. Unfortunately, for this study, budget and time constraints did not allow the production of a Dutch video or the implementation of a second pilot study.

Although interventions are the key components of trials, detailed information about the content of interventions is often not included in published reports. In general, papers on randomised controlled trials report the effect and sometimes the costeffectiveness of interventions and some also include a description of process evaluations. While these papers provide a wealth of relevant information, detailed information about the content of intervention protocols often remains inaccessible to the public domain. Availability of such detailed information about interventions is required to facilitate replication of interventions and to estimate their value. For that reason, papers about interventions should include the theoretical framework and a description of the main content, the target population, the qualifications for a facilitator (if applicable), the timeframe of the intervention, the format in which the intervention is provided to the participants, and the main intervention techniques and materials. In addition, information about the language in which the manual is written and how the complete intervention manual can be obtained should be provided. Renowned journals, however, provide limited space for information about intervention protocols in papers about intervention effects. Therefore, trial researchers should seriously consider writing separate papers with detailed information about the development and content of interventions, which may be of particular interest to practice-oriented journals.

This paper describes the development process of a Dutch version of an intervention originally developed in the US. It shows that by applying a systematic approach, like 
literally translating, consulting experts, conducting a pilot study and taking future implementation into consideration, a replication of an intervention can be developed and valuable information to improve the feasibility of the intervention for the different setting can be obtained. Therefore, we strongly recommend applying a systematic approach for the development of a replication of an intervention in a different setting. Papers about intervention protocols and the process of adapting them for different settings are scarce but nevertheless important for future application and replication of interventions. For this reason, papers reporting on interventions should provide more detailed information to facilitate replication of these interventions.

\section{Acknowledgements}

We would like to thank all experts reviewing the intervention manual, and all participants and facilitators of the pilot study for their participation and valuable comments. ZonMw The Netherlands Organisation for Health Research and Development (grant 014-91052), the Care and Public Health Research Institute and the Faculty of Health Sciences of the Maastricht University funded this project.

For information about the English version of $A M B$ please contact the Boston University Roybal Center for Enhancement of Late Life Function at www.bu.edu/roybal. For information about the Dutch version of $A M B$ (AMB-NL) please contact the corresponding author of this paper. 


\section{References}

1. Howland J, Peterson EW, Levin WC, et al. Fear of falling among the community-dwelling elderly. J Aging Health 1993;5(2):229-43.

2. Maki BE, Holliday PJ, Topper AK. Fear of falling and postural performance in the elderly. J Gerontol 1991;46(4):M123-31.

3. Tinetti ME, Speechley M, Ginter SF. Risk factors for falls among elderly persons living in the community. N Engl J Med 1988;319(26):1701-7.

4. Walker JE, Howland J. Falls and fear of falling among elderly persons living in the community: occupational therapy interventions. Am J Occup Ther 1991;45(2):119-22.

5. Vellas BJ, Wayne SJ, Romero LJ, et al. Fear of falling and restriction of mobility in elderly fallers. Age Ageing 1997;26(3):189-93.

6. Arfken CL, Lach HW, Birge SJ, et al. The prevalence and correlates of fear of falling in elderly persons living in the community. Am J Public Health 1994;84(4):565-70.

7. Friedman SM, Munoz B, West SK, et al. Falls and fear of falling: which comes first? A longitudinal prediction model suggests strategies for primary and secondary prevention. J Am Geriatr Soc 2002;50(8):1329-35.

8. Howland J, Lachman ME, Peterson EW, et al. Covariates of fear of falling and associated activity curtailment. Gerontologist 1998;38(5):549-55.

9. Murphy SL, Williams CS, Gill TM. Characteristics associated with fear of falling and activity restriction in community-living older persons. J Am Geriatr Soc 2002;50(3):516-20.

10. Stalenhoef PA, Diederiks JP, Knottnerus JA, et al. The construction of a patient recordbased risk model for recurrent falls among elderly people living in the community. Fam Pract 2000;17(6):490-6.

11. Pluijm SMF. Predictors and consequences of falls and fractures in the elderly. Vrije Universiteit Amsterdam, 2001.

12. Li F, Fisher KJ, Harmer P, et al. Fear of falling in elderly persons: association with falls, functional ability, and quality of life. J Gerontol B Psychol Sci Soc Sci 2003;58(5):P283-90.

13. Drozdick LW, Edelstein BA. Correlates of fear of falling in older adults who have experienced a fall. J Clin Geropsychol 2001;7(1):1-13.

14. Fletcher PC, Hirdes JP. Restriction in activity associated with fear of falling among community-based seniors using home care services. Age Ageing 2004;33(3):273-9.

15. Legters K. Fear of falling. Phys Ther 2002;82(3):264-72.

16. Myers AM, Powell LE, Maki BE, et al. Psychological indicators of balance confidence: relationship to actual and perceived abilities. J Gerontol A Biol Sci Med Sci 1996;51(1):M37-43.

17. Burker EJ, Wong H, Sloane PD, et al. Predictors of fear of falling in dizzy and nondizzy elderly. Psychol Aging 1995;10(1):104-10.

18. Tinetti ME, Richman D, Powell L. Falls efficacy as a measure of fear of falling. J Gerontol 1990;45(6):P239-43.

19. Vellas B, Cayla F, Bocquet $H$, de Pemille F, Albarede JL. Prospective study of restriction of activity in old people after falls. Age Ageing 1987;16(3):189-93.

20. Kressig RW, Wolf SL, Sattin RW, et al. Associations of demographic, functional, and behavioral characteristics with activity-related fear of falling among older adults transitioning to frailty. J Am Geriatr Soc 2001;49(11):1456-62. 
21. Cumming RG, Salkeld G, Thomas $M$, et al. Prospective study of the impact of fear of falling on activities of daily living, SF-36 scores, and nursing home admission. J Gerontol A Biol Sci Med Sci 2000;55(5):M299-305

22. Tinetti ME, Mendes de Leon CF, Doucette JT, et al. Fear of falling and fall-related efficacy in relationship to functioning among community-living elders. J Gerontol 1994;49(3):M140-7.

23. Mendes de Leon CF, Seeman TE, Baker DI, et al. Self-efficacy, physical decline, and change in functioning in community-living elders: a prospective study. J Gerontol B Psychol Sci Soc Sci 1996;51(4):S183-90.

24. Guideline for the prevention of falls in older persons. American Geriatrics Society, British Geriatrics Society, and American Academy of Orthopaedic Surgeons Panel on Falls Prevention. J Am Geriatr Soc 2001;49(5):664-72.

25. Brouwer BJ, Walker C, Rydahl SJ, et al. Reducing fear of falling in seniors through education and activity programs: a randomized trial. J Am Geriatr Soc 2003;51(6):829-34.

26. Tennstedt S, Howland J, Lachman M, et al. A randomized, controlled trial of a group intervention to reduce fear of falling and associated activity restriction in older adults. $J$ Gerontol B Psychol Sci Soc Sci 1998;53(6):P384-92.

27. Cameron ID, Stafford B, Cumming RG, et al. Hip protectors improve falls self-efficacy. Age Ageing 2000;29(1):57-62.

28. Tennstedt S, Peterson E, Howland J, et al. A Matter of Balance - Managing Concerns about Falls. Boston: Roybal Center Consortium, Trustees of Boston University, 1998.

29. Zijlstra GA, van Haastregt JC, van Eijk JT, et al. Evaluating an intervention to reduce fear of falling and associated activity restriction in elderly persons: design of a randomised controlled trial [ISRCTN43792817]. BMC Public Health 2005;5(1):26.

30. Bandura A. Self-efficacy: toward a unifying theory of behavioral change. Psychol Rev 1977;84(2):191-215. 
Appendix A Main contents of A Matter of Balance ${ }^{28}$

\section{Session 1: Introduction to the Programme}

Starting a group intervention

Background information on fear of falling

Beliefs and disbeliefs about fear of falling

Shifting from negative to positive thinking patterns

Finding personal solutions to deal with fear of falling

Session 2: Exploring Thoughts and Concerns about Falling

Attitudes related to fear of falling and challenging them

Adaptive responses to counter misconceptions about falls

Unhelpful thoughts and their effects regarding to feelings and behaviour

Shifting from self-defeating to self-motivating thoughts

\section{Session 3: Exercise and Fall Prevention}

Misconceptions regarding physical exercise for elderly people

Potential consequences of inactivity and benefits of physical activity

Staying or becoming physically active to prevent falls

Recognising and overcoming barriers to stay or become physically active

Appropriate physical exercises for elderly people and fall prevention

Practicing simple physical exercises

\section{Session 4: Assertiveness and Fall Prevention}

Association between assertiveness and fall prevention

Potential benefits of being assertive

Reducing fall risk by being assertive in a proper fashion

Addressing physical risk factors for falls

The influence of physical exercise on physical characteristics

Practicing physical exercises

\section{Session 5: Managing Concerns about Falling}

Developing and implementing a personal physical exercise programme

Improving one's balance

Shifting from self-defeating to self-motivating thoughts regarding physical activity and fall risk

Practicing physical exercises 
Appendix A Main contents of A Matter of Balance ${ }^{28}$ (continued)

\section{Session 6: Recognising Fall-ty Habits}

Identifying and managing risk-taking behaviour in daily life

Prioritising fall risk behaviours

Searching for suitable, personal solutions to change risk-taking behaviour into safe actions

Planning behaviour change strategies

Setting goals for activities one would like to carry out

Shifting from negative thoughts associated with planned activities to positive responses

Practicing physical exercises

Midcourse evaluation of the intervention

\section{Session 7: Recognising Fall Hazards in the Home and Community}

Potential fall hazards in homes and community

Recognising and eliminating environmental hazards by finding simple solutions

Practicing physical exercises

\section{Session 8: Practicing No Fall-ty Habits}

Practicing assertiveness skills for locating and utilising resources to prevent falls

Seeking help after a fall

Understanding that risk-taking behaviour can be eliminated

Practicing physical exercises 



\section{CHAPTER 5}

Evaluating an intervention to reduce fear of falling and associated activity restriction in elderly persons: design of a randomised controlled trial 


\section{Abstract}

Introduction Fear of falling and associated activity restriction is common in older persons living in the community. Adverse consequences of fear of falling and associated activity restriction, like functional decline and falls, may have a major impact on physical, mental and social functioning of these persons.

Objective This paper presents the design of a trial evaluating a cognitive behavioural group intervention to reduce fear of falling and associated activity restriction in older persons living in the community.

Methods A two-group randomised controlled trial was developed to evaluate the intervention. Persons 70 years of age or over and still living in the community were eligible for study if they experienced at least some fear of falling and associated activity restriction. A random community sample of older people was screened for eligibility; those eligible for study were measured at baseline and were subsequently allocated to the intervention or control group. Follow-up measurements were carried out directly after the intervention period, and then at 6 months and 12 months after the intervention. People allocated to the intervention group were invited to participate in 8 weekly sessions of 2 hours each and a booster session. This booster session was conducted before the follow-up measurement at 6 months after the intervention. People allocated to the control group received no intervention as a result of this trial. Both an effect evaluation and a process evaluation were performed. The primary outcome measures of the effect evaluation are fear of falling, avoidance of activity due to fear of falling and daily activity. The secondary outcome measures are perceived general health, selfrated life satisfaction, feelings of loneliness, activities of daily life, feelings of anxiety, symptoms of depression, social support interactions, perceived consequences of falling, perceived risk of falling, and falls. The outcomes of the process evaluation comprise performance of the intervention according to protocol, attendance and adherence of participants, and participants' and facilitators' opinion about the intervention. Data of the effect evaluation will be analysed according the intention-to-treat and on-treatment principle. Data of the process evaluation will be analysed using descriptive techniques. 


\section{Introduction}

Fear of falling and associated activity restriction are common in older persons, both in those older persons who have experienced a fall and those who have not. ${ }^{12}$ Studies in older people living in the community showed that about 20 to $60 \%$ of these persons experience at least some fear of falling ${ }^{2-6}$ and about 20 to $55 \%$ report activity restriction due to fear of falling. ${ }^{15}$ 7-9 In this paper, we focus on the design of a randomised controlled trial evaluating a cognitive behavioural group intervention, which aims to reduce fear of falling and associated activity restriction in older persons.

In one of the first reported studies on fear of falling, Vellas and colleagues indicated that fear of falling may lead to a debilitating spiral marked by loss of confidence and reduced activity, resulting ultimately in a loss of independence. ${ }^{10}$ In later studies this observation was strengthened. Fear of falling was found to be associated with several adverse factors, including decreased quality of life, ${ }^{311}$ decreased mobility, ${ }^{311}$ functional decline, ${ }^{3} 1112$ falls $^{13}$ and institutionalisation. ${ }^{11}$ These factors may not only have an adverse influence on the physical health status of older persons, but on social and mental health status as well. Therefore, reducing fear of falling and associated activity restriction in older persons may improve their health status. However, until now only a few interventions have been developed and evaluated specifically to reduce fear of falling in older people living in the community. ${ }^{14-16}$

In our trial we will evaluate one of these interventions in The Netherlands, a cognitive behavioural group intervention called 'A Matter of Balance' (AMB). This intervention has originally been developed and evaluated in the US and aims to reduce fear of falling and associated activity restriction in older persons. ${ }^{15}$ The intention-totreat analysis showed statistically significant improvements in mobility and intended activity directly after the intervention. The on-treatment analysis (including participants who attended five or more out of eight sessions) showed statistically significant improvements in falls efficacy, perceived ability to manage falls and mobility directly after the intervention. At 12 months, this latter group showed statistically significant improvements in falls efficacy, perceived ability to manage falls, mobility and social function.

Based on the reported effectiveness of AMB in the US and the aspiration to implement $A M B$ in the Dutch health care system, we decided to perform a trial to assess its effectiveness in The Netherlands. For this purpose, the intervention protocol of AMB was translated and adapted for the Dutch setting (AMB-NL). ${ }^{17}$ The current paper presents the design of a randomised controlled trial evaluating AMB-NL in Dutch older persons living in the community.

\section{Aims}

The primary aim of the effect evaluation was to study the effects of AMB-NL on fear of falling, avoidance of activity due to fear of falling and daily activity in older persons living in the community in The Netherlands. The secondary aim was to study the effects of this intervention on perceived general health, self-rated life satisfaction, feelings of loneliness, activities of daily life, feelings of anxiety, symptoms of depression, social 
support interactions, perceived consequences of falling, perceived risk of falling and falls. The aim of the process evaluation was to gain insight into factors potentially influencing the effectiveness of the intervention and factors facilitating future implementation of AMB-NL in the Dutch health care setting, if the intervention proves to be effective.

\section{Methods}

\section{Study design}

A two-group randomised controlled trial with participants being randomly allocated to either an intervention or a control group has been developed to evaluate AMB-NL. Selecting potential participants, conducting the intervention and collecting data were performed in five consecutive cycles. The first cycle started in November 2002 and the last cycle in July 2003. Each cycle lasted about 18 months and included screening for eligible participants, baseline measurement, randomisation (allocation to the intervention or control group), the intervention period, a follow-up measurement directly after the intervention period, a booster session at 6 months after the intervention, a follow-up at 6 months after the intervention (directly after the booster session) and a final follow-up at 12 months after the intervention. The Medical Ethics Committee of the Maastricht University/Academic Hospital Maastricht granted approval for conducting this trial. The study design is presented in Figure 1.

\section{Settings and locations}

Two communities, Maastricht and Heerlen, situated in the southeast of The Netherlands were selected for participation in the trial. As screening for potential participants and conducting the intervention was performed in five cycles, both communities were divided into five sections proportional to the number of potential participants in contiguous neighbourhoods. Intervention sessions were conducted in local community centres or homes for the elderly preferably located in the centre of those neighbourhoods. Transportation to the intervention location was offered to participants who expected difficulties to reach the location, for example due to health problems.

\section{Participants}

Older persons were eligible for study if they met all of the following criteria: 1) reporting at least some fear of falling; 2 ) reporting at least some associated avoidance of activity; 3) living in the community; and 4) being 70 years of age or over. Older persons confined to bed, restricted by permanent use of wheelchair or waiting for nursing home admission were excluded for study.

Immediately before screening for eligible participants in each of the five cycles, addresses of older people living in the community who were 70 years of age or over at 1 January 2003 were randomly selected by municipal registry offices. Selected persons were sent information about the trial and a brief self-administered screening questionnaire. 


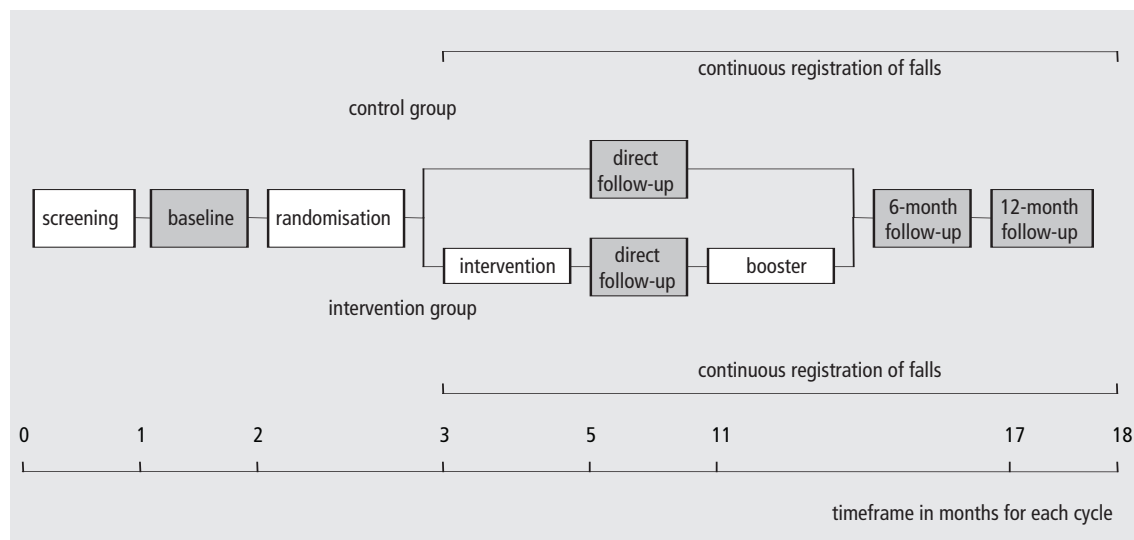

Figure 1 Study design

This questionnaire assessed socio-demographic and fall-related variables, all inclusion and exclusion criteria, and willingness to participate. Persons interested in participating in the trial were asked to sign an informed consent form enclosed in the questionnaire. All selected persons were requested to complete and return the questionnaire, even if they lacked interest in participating in the trial. A postage free envelope was enclosed for returning the questionnaire. If the questionnaire was not returned in a fortnight, a reminder letter to return the questionnaire was sent. Those persons who signed the informed consent form, were willing to participate, and met all other eligibility criteria were invited to participate in the study.

\section{Randomisation}

Randomisation was carried out directly after baseline measurement and was performed per community to ensure having both an intervention group and a control group in each of the two communities. During each cycle two intervention groups were composed per community. Per cycle, approximately half of the participants were allocated to the intervention group (with a maximum of 15 participants per intervention group). Participants allocated to the intervention group were then randomly allocated to one of the two groups in their own community. Participants allocated to the control group received no intervention as a result of this trial. An independent researcher conducted randomisation by selecting random samples using SPSS 12.0 for Windows.

\section{Intervention}

The intervention AMB-NL is a translated and adapted version ${ }^{17}$ of a cognitive behavioural group intervention for older persons living in the community developed by Tennstedt and colleagues. ${ }^{15}$ This intervention has been developed to reduce fear of falling and promote physical, social and functional activity in older persons living in the community. Principles of cognitive restructuring ${ }^{18}$ are applied by focusing on changing attitudes and self-efficacy beliefs with respect to falling before attempting to change actual behaviour. To attain a reduction in fear of falling, the intervention aims to increase 
self-efficacy beliefs with regard to falling as well as the sense of control over falling. Four strategies are used to accomplish these aims: (1) restructuring misconceptions to promote a view of fall risk and fear of falling as controllable; (2) setting realistic goals for increasing activity; (3) changing the environment to reduce fall risk; and (4) promoting physical exercise to increase strength and balance. ${ }^{15}$

The intervention consists of 8 weekly group sessions lasting 2 hours. Six months after the eighth session a booster session is scheduled. The main topics in each of the sessions of AMB-NL, presented in Table 1, were discussed similarly: (1) introduction; (2) participant's point of view; (3) positive and negative aspects concerning the topic; (4) association with falls or fear of falling; and (5) implementation in the participant's daily life. A more extensive description of the intervention is published elsewhere. ${ }^{17}$

Nurses qualified in the field of geriatrics and working for home care agencies were trained as facilitators of the intervention. Except for the first session when two facilitators were present, each intervention session was conducted by one facilitator. Monthly meetings with facilitators and researchers were scheduled to evaluate and discuss the progress of the trial, the intervention and associated matters. Participants were informed to notify the facilitators or researchers if they were unable to attend a session. After the session, facilitators contacted those participants who were absent and briefly discussed the topics of that session. However, those participants who were absent in all of the first three sessions were excluded from further participation in the intervention due to an unbridgeable deficiency in knowledge.

Table 1 Main contents of the Dutch version of AMB (AMB-NL)

\section{Session 1: Introduction to the Programme}

Starting a group intervention (e.g., getting acquainted)

Background information on fear of falling (e.g., incidence, impact)

Beliefs and disbeliefs about fear of falling

Shifting from negative to positive thinking patterns

Finding personal solutions to deal with fear of falling

\section{Session 2: Exploring Thoughts and Concerns about Falling}

Attitudes related to fear of falling and challenging them

Adaptive responses to counter misconceptions about falls

Unhelpful thoughts and their effects regarding to feelings and behaviour

Shifting from self-defeating to self-motivating thoughts

\section{Session 3: Exercise and Fall Prevention}

Misconceptions regarding physical exercise for elderly people

Potential consequences of inactivity and benefits of physical activity

Staying or becoming physically active to prevent falls

Recognising and overcoming barriers to stay or become physically active

Appropriate physical exercises for elderly people and fall prevention

Practicing simple physical exercises 
Table 1 Main contents of the Dutch version of AMB (AMB-NL) (continued)

\author{
Session 4: Assertiveness and Fall Prevention \\ Association between assertiveness and fall prevention \\ Potential benefits of being assertive \\ Reducing fall risk by being assertive in a proper fashion \\ Addressing physical risk factors for falls \\ The influence of physical exercise on physical characteristics (e.g., blood pressure) \\ Practicing physical exercises
}

\title{
Session 5: Managing Concerns about Falling
}

Developing and implementing a personal physical exercise programme

Shifting from self-defeating to self-motivating thoughts regarding physical activity and fall risk

Practicing physical exercises

Midcourse evaluation to review all main topics

\section{Session 6: Recognising Fall-ty Habits}

Identifying and managing risk-taking behaviour in daily life

Prioritising fall risk behaviours

Searching for suitable, personal solutions to change risk-taking behaviour into safe actions

Planning behaviour change strategies

Setting goals for activities one would like to carry out

Shifting from negative thoughts associated with planned activities to positive responses

Practicing physical exercises

Discussing falls and seeking help after a fall

\section{Session 7: Recognising Fall Hazards in the Home and Community}

Potential fall hazards in homes and community

Recognising and eliminating environmental hazards by finding simple solutions

Discussing displayed assistive devices which improve safety

Practicing physical exercises

\section{Session 8: Practicing No Fall-ty Habits}

Practicing assertiveness skills for locating and utilising resources to prevent falls

Understanding that risk-taking behaviour can be eliminated

Practicing physical exercises

\section{Booster session}

Discussing personal experiences with falls and fear of falling

Shifting from self-defeating to self-motivating thoughts

Exercise and fall prevention

Potential fall hazards in homes and community

Change risk-taking behaviour into safe actions

Practicing physical exercises 


\section{Measures}

\section{Effect evaluation}

\section{Primary outcome variables}

The primary outcomes of the effect evaluation are fear of falling, avoidance of activity due to fear of falling and daily activity. Fear of falling was assessed by three different measures. First, respondents indicated the frequency of fear of falling when asked "Are you afraid of falling?" ( 1 = never to $5=$ very often). Second, on a 14-item falls efficacy scale respondents indicated how concerned they are about falling while carrying out several activities of daily living ( $1=$ not at all concerned to $4=$ very concerned). ${ }^{7} 1920$ Finally, a four-item scale was used to assess the respondent's perceived control over falling. ${ }^{21}$ This scale focuses on the perceived control over the environment and one's own mobility and ability to do things to prevent falls and reduce fear of falling. The frequency of avoidance of activity due to fear of falling was assessed by the question "Do you avoid certain activities due to fear of falling?" ( 1 = never to 5 = always). Daily activity was assessed by the Frenchay Activities Index.22 23 This index measures the frequency in which daily activities that reflect the broader everyday activities of normal living are performed. ${ }^{22}$ An overview of the primary and secondary outcomes measured during the course of the study is presented in Table 2.

\section{Secondary outcome variables}

The secondary outcomes that were assessed are: perceived general health (item one of the MOS SF-20), ${ }^{24} 25$ self-rated life satisfaction (seven-point satisfaction rating), ${ }^{26}$ feelings of loneliness (six-point Likert scale), activities of daily life (ADL subscale of the Groningen Activity Restriction Scale), ${ }^{27}$ feelings of anxiety (anxiety subscale of the Hospital Anxiety and Depression Scale), ${ }^{28} 29$ symptoms of depression (depression subscale of the Hospital Anxiety and Depression Scale), ${ }^{2829}$ social support interactions, ${ }^{30}$ perceived consequences of falling - loss of functional independence subscale and damage to identity subscale, ${ }^{31}$ perceived risk of falling and falls. Feelings of loneliness were assessed by the question "During the past 4 weeks, how often did you feel lonely?" ( 1 = all the time to 6 = never). Falls were registered by both a one-item question and a fall calendar. The one-item question assessed how frequently the participant had fallen during the past months ( $1=$ never to $6=5$ or more falls). The fall calendar was used for continuous registration of falls during the course of the trial. If a fall occurred, participants indicated on the calendar: (a) the location of the fall (indoor or outdoor); and (b) the number of times medical care was received due to the fall.

\section{Additional variables}

Several additional variables were assessed to provide insight into the population, to interpret the outcomes of the trial and to study the underlying mechanisms of the intervention. Socio-demographic variables assessed during the process of screening were: age, gender, marital status, living situation, living alone or not, and educational level. Health-related variables assessed during the telephone interview of the baseline measurement were: chronic medical conditions (a 19-item checklist), ${ }^{32}$ cognitive status (modified version of the Telephone Interview for Cognitive Status), ${ }^{33}$ and impaired vision and hearing (a four-item questionnaire). ${ }^{34}$ Other health-related variables assessed 
Table 2 Primary and secondary outcome measures of the effect evaluation

\begin{tabular}{|c|c|c|c|c|c|c|c|}
\hline Variables & $\begin{array}{l}\text { No. of } \\
\text { items }\end{array}$ & Range* & $S$ & B & FU1 & FU2 & FU3 \\
\hline \multicolumn{8}{|l|}{ Primary outcome measures } \\
\hline fear of falling & 1 & 1 to 5 & SQ & Q & $Q$ & Q & Q \\
\hline fall-related self-efficacy7 1920 & 14 & $\underline{14}$ to 56 & - & TI & TI & $\mathrm{TI}$ & TI \\
\hline perceived control over falling ${ }^{21}$ & 4 & 4 to $\underline{20}$ & - & Q & Q & Q & Q \\
\hline avoidance of activity due to fear of falling & 1 & 1 to 5 & SQ & $Q$ & Q & Q & Q \\
\hline daily activity 2223 & 15 & 15 to $\underline{60}$ & - & Q & Q & Q & Q \\
\hline \multicolumn{8}{|l|}{ Secondary outcome measures } \\
\hline perceived general health 2425 & 1 & 1 to 5 & SQ & Q & $Q$ & Q & Q \\
\hline self-rated life satisfaction ${ }^{26}$ & 1 & 1 to $\underline{7}$ & - & Q & Q & Q & Q \\
\hline feelings of loneliness & 1 & 1 to $\underline{6}$ & - & $Q$ & Q & Q & Q \\
\hline activities of daily life ${ }^{27}$ & 11 & $\underline{11}$ to 44 & - & TI & TI & TI & TI \\
\hline feelings of anxiety ${ }^{28} 29$ & 7 & $\underline{0}$ to 21 & - & $Q$ & Q & Q & $Q$ \\
\hline symptoms of depression 2829 & 7 & $\underline{0}$ to 21 & - & Q & Q & Q & Q \\
\hline social support interactions ${ }^{30}$ & 12 & 12 to $\underline{48}$ & - & $Q$ & Q & Q & Q \\
\hline no of falls in the previous 6 months & 1 & 1 to 6 & SQ & - & - & Q & Q \\
\hline no of falls in the previous 2 months & 1 & 1 to 6 & - & Q & Q & - & - \\
\hline no of indoor falls and number of outdoor falls & 1 & N/A & - & $C>$ & $C>$ & $C>$ & $C>$ \\
\hline no of times medical attention required due to falls & 1 & N/A & - & $C>$ & $C>$ & C> & $C>$ \\
\hline CoF - loss of functional independence ${ }^{31}$ & 6 & $\underline{6}$ to 24 & - & $\mathrm{Tl}$ & $\mathrm{Tl}$ & $\mathrm{Tl}$ & $\mathrm{TI}$ \\
\hline $\mathrm{CoF}$ - damage to identity ${ }^{31}$ & 6 & $\underline{6}$ to 24 & - & $\mathrm{TI}$ & TI & TI & TI \\
\hline perceived risk of falling & 3 & $\underline{3}$ to 12 & - & $\mathrm{TI}$ & TI & TI & $\mathrm{Tl}$ \\
\hline
\end{tabular}

at baseline and follow-up telephone interviews were: use of health care (for example, number of visits to the general practitioner) and use of assistive devices. Additional data assessed with baseline and follow-up questionnaires were: general self-efficacy, ${ }^{35} 36$ physical self-efficacy, ${ }^{37}$ social self-efficacy ${ }^{35}$ and mastery. ${ }^{38}$ An overview of the additional health-related scales, and self-efficacy and mastery scales is presented in Table 3.

\section{Process evaluation}

The process evaluation was aimed at gaining insight into factors potentially influencing the effectiveness of the intervention and factors facilitating future implementation of the intervention. Four main outcome measures were identified: (1) performance of the intervention according to protocol; (2) attendance of participants; (3) adherence of participants; and (4) opinion of participants and facilitators about the intervention. Table 4 provides an overview of outcomes of the process evaluation during the course of the trial. 
Table 3 Additional measures during the trial

\begin{tabular}{lcccccc} 
Variables & No. of items & Range* $^{*}$ & B & FU1 & FU2 & FU3 \\
\hline chronic medical conditions $^{32}$ & 19 & $\underline{0}$ to 19 & TI & - & - & - \\
cognitive status $^{33}$ & 11 & 0 to $\underline{41}$ & TI & - & - & - \\
impaired vision $^{34}$ & 2 & $\underline{2}$ to 8 & TI & - & - & - \\
impaired hearing $^{34}$ & 2 & $\underline{2}$ to 8 & TI & - & - & - \\
use of health care & 6 & N/A & TI & TI & TI & TI \\
use of assistive devices & 14 & N/A & TI & TI & TI & TI \\
general self-efficacy 3536 & 16 & 16 to $\underline{80}$ & Q & Q & Q & Q \\
physical self-efficacy $^{37}$ & 10 & 10 to $\underline{50}$ & Q & Q & Q & Q \\
social self-efficacy $^{35}$ & 6 & 6 to $\underline{\underline{30}}$ & Q & Q & Q & Q \\
mastery $^{38}$ & 7 & 7 to $\underline{\underline{35}}$ & Q & Q & Q & Q
\end{tabular}

* The underlined scores indicate the most favourable scores; $N / A=$ not applicable. $B=$ baseline; FU1 = direct follow-up; $\mathrm{FU} 2$ = 6-month follow-up; FU3 = 12-month follow-up; $\mathrm{Q}=$ questionnaire; $\mathrm{Tl}=$ telephone interview.

\section{Data collection}

Data for the effect evaluation were gathered by means of self-administered questionnaires, fall calendars and telephone interviews. Trained interviewers, who were blinded for group allocation, conducted the interviews. After baseline measurement participants received a fall calendar; every 3 months a sheet of the calendar was to be returned to the research team. Data for the process evaluation collected from the participants were completed by means of self-administered questionnaires and short telephone interviews. Registration forms and self-administered questionnaires were used to gather data from the facilitators. These data were discussed and illustrated by the facilitators in two evaluative meetings.

As recommended by Hollis and Campbell, ${ }^{39}$ non-compliant participants of the intervention group were approached for all follow-up measurements and participants with missing data were contacted to ensure completion of data. At 5 and 11 months after the intervention, newsletters were sent to keep the participants interested in participating in the trial and to notify them about its progress.

\section{Sample size and power}

Sample size calculations based on previous experiences in evaluative studies among older persons in The Netherlands ${ }^{40}$ and the US ${ }^{15}$ showed that 92 participants were needed in each group to detect a mean difference of at least 2.5 points on fall-related self-efficacy based on an alpha of $5 \%$ (two-sided) and power of $80 \%$. Taking a dropout rate of $35 \%$ into account, at least 142 participants were required for each group. Based on unpublished data of Van Haastregt and colleagues (study described in ${ }^{40}$ ), we estimated that approximately 14.5 percent of the older persons who returned the screening questionnaire would meet all inclusion criteria and would be interested in participating in the trial. With an estimated response rate of 55 percent, a minimum sample of 3,560 older persons aged 70 or over needed to be approached with a screening questionnaire. 
Table 4 Outcome measures of the process evaluation

\begin{tabular}{|c|c|c|c|c|}
\hline Variables & $\mathrm{BDI}$ & FU1 & FU2 & FU3 \\
\hline \multicolumn{5}{|l|}{ Performance intervention according to protocol } \\
\hline duration of the sessions & $R^{f}$ & - & - & - \\
\hline deviations from protocol & $R^{f}$ & - & - & - \\
\hline \multicolumn{5}{|l|}{ Participant attendance } \\
\hline reasons for refusal before the start of the intervention & $\mathrm{TI}^{\mathrm{P}}$ & - & - & - \\
\hline number of sessions visited by each subject & $R^{f}$ & - & $R^{f}$ & - \\
\hline reasons for stopping during the intervention period & $\mathrm{TIP}$ & - & $\mathrm{TI}$ & - \\
\hline \multicolumn{5}{|l|}{ Participant adherence } \\
\hline adherence to homework assignments & - & $Q^{p /} Q^{f}$ & - & - \\
\hline adherence to physical exercise & - & $Q^{P} / Q^{f}$ & $Q^{p}$ & $Q^{p}$ \\
\hline \multicolumn{5}{|l|}{ Opinion about intervention } \\
\hline overall opinion of the intervention (grade) & - & $Q^{P} / Q^{f}$ & - & - \\
\hline opinion of the facilitators (grade) & - & $Q^{P} / Q^{f}$ & - & - \\
\hline benefits experienced by participants & - & $Q^{p} / Q^{f}$ & $Q^{p}$ & $Q^{p}$ \\
\hline strong and weak aspects of the intervention & - & $Q^{P} / Q^{f}$ & - & - \\
\hline suggestions for improvement & - & $Q^{P} / Q^{f}$ & $Q^{f}$ & - \\
\hline
\end{tabular}

\section{Analysis}

Descriptive techniques will be used to describe participants of the trial. To detect differences between intervention and control group at the start of the trial, baseline variables will be compared. Baseline variables of compliant and non-compliant participants of the intervention group will be compared as well. Compliant participants are participants who attended five or more sessions, ${ }^{15}$ not including the booster session.

Data of the effect evaluation will be analysed according the intention-to-treat and ontreatment principle. Univariate and multivariate techniques will be applied to examine differences in the intervention and control group with regard to the primary and secondary outcome measures at the follow-up measurements. Effect sizes ${ }^{41}$ will be calculated to quantify the size of the difference between both groups. Subgroup analysis will be performed with several potential effect modifiers, like cognitive status or educational level. Data of the process evaluation will be analysed using descriptive techniques.

\section{Discussion}

\section{Progress of the study}

A random selection of older persons aged 70 or over living in the community was screened for eligibility in five cycles between November 2002 and July 2003. A total of 7,431 older persons rightfully received the screening questionnaire. The response rate 
was 58.9 percent. During the course of the study the number of people who were sent a screening questionnaire was increased based on two grounds. First, in contrast to our estimate of 14.5 percent, only about 10 percent of the participants in the first cycle met our eligibility criteria and were willing to participate. Second, about 25 percent of the persons participating in the baseline measurement dropped out before randomisation. In total, 540 participants were included in the trial; 260 participants were allocated to the intervention group and 280 to the control group. The data collection was completed in February 2005. Currently, preparations for analysing the data of the screening process and process evaluation are made. Data of the effect evaluation will be available in 2005 .

\section{Future implementation}

Implementation in the Dutch setting has been taken into account throughout the development of AMB-NL. ${ }^{17}$ If the results of this trial show the effectiveness of AMB$\mathrm{NL}$, recommendations will be developed to implement the intervention in the Dutch health care setting and a manual of the intervention, updated with the experiences of the trial, will be made available.

\section{Acknowledgements}

We would like to thank ZonMw - The Netherlands Organisation for Health Research and Development (grant 014-91-052), the Care and Public Health Research Institute, and the Faculty of Health, Medicine and Life Sciences of the Maastricht University for funding this study. 


\section{References}

1. Howland J, Peterson EW, Levin WC, et al. Fear of falling among the community-dwelling elderly. J Aging Health 1993;5(2):229-43.

2. Tinetti ME, Speechley M, Ginter SF. Risk factors for falls among elderly persons living in the community. N Engl J Med 1988;319(26):1701-7.

3. Arfken CL, Lach HW, Birge SJ, et al. The prevalence and correlates of fear of falling in elderly persons living in the community. Am J Public Health 1994;84(4):565-70.

4. Howland J, Lachman ME, Peterson EW, et al. Covariates of fear of falling and associated activity curtailment. Gerontologist 1998;38(5):549-55.

5. Murphy SL, Williams CS, Gill TM. Characteristics associated with fear of falling and activity restriction in community-living older persons. J Am Geriatr Soc 2002;50(3):516-20.

6. Stalenhoef PA, Diederiks JP, Knottnerus JA, et al. The construction of a patient recordbased risk model for recurrent falls among elderly people living in the community. Fam Pract 2000;17(6):490-6.

7. Tinetti ME, Richman D, Powell L. Falls efficacy as a measure of fear of falling. J Gerontol 1990;45(6):P239-43.

8. Tinetti ME, Mendes de Leon CF, Doucette JT, et al. Fear of falling and fall-related efficacy in relationship to functioning among community-living elders. J Gerontol 1994;49(3):M140-7.

9. Fletcher PC, Hirdes JP. Restriction in activity associated with fear of falling among community-based seniors using home care services. Age Ageing 2004;33(3):273-9.

10. Vellas B, Cayla F, Bocquet $\mathrm{H}$, et al. Prospective study of restriction of activity in old people after falls. Age Ageing 1987;16(3):189-93.

11. Cumming RG, Salkeld G, Thomas $M$, et al. Prospective study of the impact of fear of falling on activities of daily living, SF-36 scores, and nursing home admission. J Gerontol A Biol Sci Med Sci 2000;55(5):M299-305.

12. Mendes de Leon CF, Seeman TE, Baker DI, et al. Self-efficacy, physical decline, and change in functioning in community-living elders: a prospective study. J Gerontol B Psychol Sci Soc Sci 1996;51(4):S183-90.

13. Friedman SM, Munoz B, West SK, et al. Falls and fear of falling: which comes first? A longitudinal prediction model suggests strategies for primary and secondary prevention. J Am Geriatr Soc 2002;50(8):1329-35.

14. Brouwer BJ, Walker C, Rydahl SJ, et al. Reducing fear of falling in seniors through education and activity programs: a randomized trial. J Am Geriatr Soc 2003;51(6):829-34.

15. Tennstedt S, Howland J, Lachman M, et al. A randomized, controlled trial of a group intervention to reduce fear of falling and associated activity restriction in older adults. $J$ Gerontol B Psychol Sci Soc Sci 1998;53(6):P384-92.

16. Cameron ID, Stafford B, Cumming RG, et al. Hip protectors improve falls self-efficacy. Age Ageing 2000;29(1):57-62.

17. Zijlstra GA, Tennstedt SL, Haastregt JC, et al. Reducing fear of falling and avoidance of activity in elderly persons: The development of a Dutch version of an American intervention. Patient Educ Couns 2006;62(2):220-7.

18. Lachman ME, Weaver SL, Bandura M, et al. Improving memory and control beliefs through cognitive restructuring and self-generated strategies. J Gerontol 1992;47(5):P293-9. 
19. Tinetti ME, Baker DI, McAvay G, et al. A multifactorial intervention to reduce the risk of falling among elderly people living in the community. N Engl J Med 1994;331(13):821-7.

20. Hill KD, Schwarz JA, Kalogeropoulos AJ, et al. Fear of falling revisited. Arch Phys Med Rehabil 1996;77(10):1025-9.

21. Lawrence RH, Tennstedt SL, Kasten LE, et al. Intensity and correlates of fear of falling and hurting oneself in the next year: baseline findings from a Roybal Center fear of falling intervention. J Aging Health 1998;10(3):267-86.

22. Holbrook M, Skilbeck CE. An activities index for use with stroke patients. Age Ageing 1983;12(2):166-70.

23. Schuling J, de Haan R, Limburg M, Groenier KH. The Frenchay Activities Index. Assessment of functional status in stroke patients. Stroke 1993;24(8):1173-7.

24. Stewart AL, Hays RD, Ware JE, Jr. The MOS short-form general health survey. Reliability and validity in a patient population. Med Care 1988;26(7):724-35.

25. Kempen GI, Brilman El, Heyink JW, et al. Het meten van de algemene gezondheidstoestand met de MOS Short-Form General Health Survey (SF-20): een handleiding. Groningen: Noordelijk Centrum voor Gezondheidsvraagstukken, Rijksuniversiteit Groningen, 1995.

26. Andrews FM, Whitney SB. Social indicators of well-being. New York: Plenum Press, 1976.

27. Kempen GI, Miedema I, Ormel J, et al. The assessment of disability with the Groningen Activity Restriction Scale: Conceptual framework and psychometric properties. Soc Sci Med 1996;43(11):1601-10.

28. Bjelland I, Dahl AA, Haug T, et al. The validity of the Hospital Anxiety and Depression Scale. An updated literature review. J Psychosom Res 2002;52(2):69-77.

29. Spinhoven PH, Ormel J, Sloekers PP, et al. A validation study of the Hospital Anxiety and Depression scale (HADS) in different groups of Dutch subjects. Psychol Med 1997;27(2):363-70.

30. Kempen GI, Van Eijk LM. The psychometric properties of the SSL12-I, a short scale for measuring social support in the elderly. Soc Indic Res 1995;35(3):303-12.

31. Yardley L, Smith H. A prospective study of the relationship between feared consequences of falling and avoidance of activity in community-living older people. Gerontologist 2002;42(1):17-23.

32. CBS. Gezondheidsenquete 1989 (Health Interview Survey). Voorburg/Heerlen, The Netherlands, 1989.

33. Brandt J, Spencer M, Folstein M. The Telephone Interview for Cognitive Status. Neuropsychiatry Neuropsychol Behav Neurol 1988;1(2):111-17.

34. van Sonsbeek JLA. Methodological aspects and content of the OECD-longterm disability indicator. CBS Maandberichten Gezondheid 1988;6:4-17.

35. Sherer M, Maddux JE. The Self-Efficacy Scale: Construction and Validation. Psychol Rep 1982;51:663-71.

36. Bosscher RJ, Smit JH. Confirmatory factor analysis of the General Self-Efficacy Scale. Behav Res Ther 1998;36(3):339-43.

37. Bosscher RJ, Laurijssen L, de Boer E. Measuring physical self-efficacy in old age. Percept Mot Skills 1993;77(2):470.

38. Pearlin LI, Schooler C. The structure of coping. J Health Soc Behav 1978;19(1):2-21.

39. Hollis $S$, Campbell F. What is meant by intention to treat analysis? Survey of published randomised controlled trials. BMJ 1999;319(7211):670-4. 
40. van Haastregt JC, Diederiks JP, van Rossum E, et al. Effects of a programme of multifactorial home visits on falls and mobility impairments in elderly people at risk: randomised controlled trial. BMJ 2000;321(7267):994-8.

41. Cohen J. A power primer. Psychol Bull 1992;112(1):155-9. 



\section{CHAPTER 6}

Feasibility of a cognitive behavioural group intervention to reduce fear of falling and associated avoidance of activity in community-living older people: a process evaluation 


\section{Abstract}

Introduction Fear of falling and associated avoidance of activity are common among older people and may have negative consequences in terms of functional decline, quality of life and institutionalisation. We evaluated the effects of a cognitive behavioural group intervention to reduce fear of falling and associated avoidance of activity among older persons. This intervention showed favourable effects on fear of falling, avoidance of activity, daily activity and several secondary outcomes. The aim of the present study is to assess the feasibility of this cognitive behavioural group intervention for participants and facilitators.

Methods The intervention consisted of 8 weekly group sessions lasting 2 hours each and a booster session after 6 months. Self-administered questionnaires, registration forms and interviews were used to collect data from participants $(n=168)$ and facilitators $(n=6)$ on the extent to which the intervention was performed according to protocol, participant attendance, participant adherence, and participants' and facilitators' opinion of the intervention. Quantitative data from the questionnaires and registration forms were analysed by means of descriptive statistics. Qualitative data were categorised based on matching contents of the answers.

Results Facilitators reported no major protocol deviations. Twenty-six percent of the participants withdrew before the start of the programme. Of the persons who started the programme, $84 \%$ actually completed it. The participants reported their adherence as good, but facilitators had a less favourable opinion of this. The majority of participants still reported substantial benefits from the programme after 6 and 12 months of followup (71 and 61\%, respectively). Both participants and facilitators provided suggestions for improvement of the intervention.

Conclusions Results of this study show that the current cognitive behavioural group intervention is feasible for both participants and facilitators, and fits in well with regular care. Minor refinement of the intervention, however, is warranted to further improve intervention effectiveness and efficiency. Based on these positive findings, we recommend implementing a refined version of this effective and feasible intervention in regular care. 


\section{Introduction}

Fear of falling and associated activity restriction are common problems among older people. About 20 to $60 \%$ of older people living in the community are afraid to fall1-5 and 20 to 55\% report activity restriction due to fear of falling. ${ }^{5-9}$ Fear of falling and associated avoidance of activity may have negative consequences in terms of functional decline, decreased quality of life and institutionalisation. ${ }^{21011}$ For this reason, Tennstedt and colleagues developed a cognitive behavioural group intervention primarily aimed at reducing fear of falling and avoidance of activity. ${ }^{12}{ }^{13}$ This intervention, called 'A Matter of Balance', showed favourable effects on falls efficacy, perceived ability to manage falls, mobility, intended activity and social functioning in the US. ${ }^{12}$ Success of an intervention in the US is, however, no guarantee for its success in other countries. Therefore, we translated the protocol of this intervention into Dutch and made some adjustments to the protocol based on recommendations by experts, and experiences in the US and a pilot study. ${ }^{14}$ Next, we carried out a randomised controlled trial to assess both short-term and long-term effects of 'A Matter of Balance' in The Netherlands. ${ }^{15}$

${ }^{16}$ Results of this trial showed that the intervention had statistically significant favourable effects on all primary outcomes: fear of falling, avoidance of activity and daily activity. Furthermore, favourable effects were observed on several secondary outcomes. ${ }^{17}$

As has been widely acknowledged, a detailed process evaluation should be integral to the design of randomised controlled trials. ${ }^{18} 19$ Process evaluations may facilitate interpretation of outcomes, recognition of strong and weak aspects of the intervention, and implementation of the intervention. In the present paper we discuss the results of a process evaluation, which was performed alongside the trial. ${ }^{15} 17$ The aim of the present process evaluation is to study the feasibility of the intervention by assessing: a) the extent to which facilitators reported that the intervention was performed according to protocol; b) participant attendance; c) participant adherence; and d) participants' and facilitators' opinion of the intervention.

\section{Methods}

\section{Study design and population}

The current process evaluation is a descriptive study with longitudinal elements, in which quantitative and qualitative data were gathered. Our study population consisted of 280 older persons who received a cognitive behavioural intervention ${ }^{15}$ and six facilitators who conducted this intervention. All 280 participants were community-living, aged 70 years or older and had reported at least some fear of falling and related avoidance of activity in a screening questionnaire. ${ }^{15} 16$ This questionnaire was sent to a random sample of 7,431 older persons living independently in either Maastricht or Heerlen in the southeast of The Netherlands. ${ }^{15} 16$ The time period between receiving the screening questionnaire and starting the intervention was about 3 months. The facilitators were community nurses, specialised in geriatric care and employed with two local home care organisations, who received two days' training before conducting the intervention. Details about study design and population are reported elsewhere. ${ }^{15} 16$ 


\section{Intervention}

The intervention is a cognitive behavioural group programme developed to reduce fear of falling and to promote activity among older persons living in the community. The intervention consists of 8 weekly sessions lasting 120 minutes and a booster session at 6 months after the end of the intervention lasting 135 minutes. Two facilitators conduct the first session; one facilitator conducts the next seven sessions and the booster session. The extra facilitator in the first session serves as a substitute for the first facilitator in case of his or her absence.

The intervention employs the following strategies to reduce fear of falling and activity restriction: a) restructuring misconceptions to promote the view that the risk of falls and fear of falling are controllable; b) setting realistic goals for increasing activity; c) changing the environment to reduce the risk of falls; and d) promoting physical exercise to increase strength and balance. The themes of the eight sessions are presented in Table 1 and are described elsewhere in more detail. ${ }^{14}$

A variety of didactic techniques is used during the sessions, including lectures, videos, group discussions, mutual problem-solving, physical exercises and skill development. The participants are given homework at the end of each session. This homework includes reading informative handouts, challenging concerns about falling on pre-structured forms, filling in home safety checklists and filling in personal action planners. In addition, facilitators encourage participants to practise the physical exercises at home both during and after the programme. The exercises are taught during the sessions and are described in detail in illustrated handouts. More information about the intervention protocol can be obtained from the authors. ${ }^{14}$

Between February 2003 and May 2004, 20 groups received the intervention provided by the six facilitators. Each group consisted of approximately 10 participants. Participants who were unable to come to the programme location independently were offered free transportation by taxi.

Table 1 Dutch version of A Matter of Balance

\section{Session themes}

1. Introduction to the programme

2. Exploring thoughts and concerns about falling

3. Exercise and fall prevention

4. Assertiveness and fall prevention

5. Managing concerns about falling

6. Recognising fall-ty habits

7. Recognising fall hazards in the home and community

8. Practicing no fall-ty habits

9. Booster session

Note: a more detailed description is presented elsewhere. ${ }^{14} 15$ 


\section{Data collection}

To study the feasibility of the intervention the following process outcomes were assessed: the extent to which facilitators reported that the intervention was performed according to protocol, participant attendance, participant adherence, and participants' and facilitators' opinion of the intervention (Table 2). Data were collected from participants by means of self-administered questionnaires and short interviews by telephone. Participants received a questionnaire directly after the last session of the programme (FU1), and at 6 (FU2) and 12 (FU3) months after the programme. The questionnaires were sent only to those who had completed the programme (i.e. persons who had not withdrawn during the eight intervention sessions). Registration forms and self-administered questionnaires were used to collect data from the facilitators. They were asked to fill in a registration form after each session including questions about time spent on performing the intervention, performance according to protocol, nature of and reasons for protocol deviations, adherence of the group during the session, and strong and weak aspects of the session. Facilitators also received an overall evaluative questionnaire directly after the end of the programme and after the booster session. In addition, the facilitators discussed and explained their written reports in two group meetings to conclude and evaluate the intervention.

The following background characteristics were gathered before randomisation by means of self-administered questionnaires: age, gender, living alone or not, educational level, cognitive status (Dutch version of the Telephone Interview for Cognitive Status), use of walking aids, perceived general health (item one of the MOS SF-20), fear of falling

Table 2 Outcome measures of the process evaluation

\begin{tabular}{|c|c|c|c|c|}
\hline Variables & BDI & FU1 & FU2 & FU3 \\
\hline \multicolumn{5}{|l|}{ Performance intervention according to protocol } \\
\hline duration of the sessions & $R^{f}$ & - & - & - \\
\hline deviations from protocol & $R^{f}$ & - & - & - \\
\hline \multicolumn{5}{|l|}{ Participant attendance } \\
\hline reasons for refusal before the start of the intervention & $\mathrm{TIP}$ & - & - & - \\
\hline number of sessions visited by each subject & $\mathrm{R}^{f}$ & - & $R^{f}$ & - \\
\hline reasons for stopping during the intervention period & $\mathrm{TI}^{\mathrm{p}}$ & - & $\mathrm{TI}^{\mathrm{p}}$ & - \\
\hline \multicolumn{5}{|l|}{ Participant adherence } \\
\hline adherence to homework assignments & - & $Q^{p} / Q^{f}$ & - & - \\
\hline adherence to physical exercise & - & $Q^{p} / Q^{f}$ & $Q^{p}$ & $Q^{p}$ \\
\hline \multicolumn{5}{|l|}{ Opinion about intervention } \\
\hline overall opinion of the intervention (grade) & - & $Q^{p} / Q^{f}$ & - & - \\
\hline opinion of the facilitators (grade) & - & $Q^{p} / Q^{f}$ & - & - \\
\hline benefits experienced by participants & - & $Q^{p} / Q^{f}$ & $Q^{p}$ & $Q^{p}$ \\
\hline strong and weak aspects of the intervention & - & $Q^{p} / Q^{f}$ & - & - \\
\hline suggestions for improvement & - & $Q^{p} / Q^{f}$ & $Q^{f}$ & - \\
\hline
\end{tabular}


("Are you afraid of falling?"; 1 = never to 5 = very often) and avoidance of activity due to fear of falling ("Do you avoid certain activities due to fear of falling?"; 1 = never to 5 = very often). ${ }^{15}$ All data were gathered in the period between February 2003 and June 2004. The intervention and measurement instruments were pre-tested in a pilot study among 10 persons who met the inclusion criteria. ${ }^{14}$

\section{Data analysis}

Quantitative data from the questionnaires and registration forms were analysed by means of descriptive statistics. Qualitative data from the questionnaires and registration forms (i.e. the answers to open questions) were categorised until themes and patterns in the answers emerged. The discussions of the facilitators during the group meetings were recorded on audiotape and transcribed. Relevant information resulting from these two meetings was used to facilitate the interpretation of the results of the questionnaires and registration forms filled in by the facilitators.

This study, which is part of a larger study, was approved by the Medical Ethics Committee of Maastricht University/University Hospital Maastricht. All participants signed an informed consent form.

\section{Results}

\section{Response}

A total of 174 persons (74\%) had completed the programme and received the first evaluation questionnaire immediately after the programme (Figure 1). Six questionnaires were not returned, resulting in a response of $97 \%(n=168)$. Of the 161 persons who did not withdraw in the period between the end of the programme and the booster session after 6 months, 159 (99\%) filled in the second questionnaire at FU2 and 151 (94\%) the third at FU3.

Table 3 shows the characteristics of the 174 persons who completed the intervention and the characteristics of the 106 persons who did not complete the intervention. Overall the persons completing the intervention seem to be less frail, less afraid of falling and less avoidant than the persons who did not complete the intervention.

Facilitators filled in registration forms for all but one of the 160 programme sessions (20 groups $x 8$ sessions) and for all the booster sessions $(n=20)$. All facilitators filled in the evaluation questionnaires after the end of the programme and after the booster session, and all facilitators participated in at least one of the two group meetings.

\section{Implementation of intervention according to protocol}

Mean duration of the sessions was 123 minutes for the programme sessions and 135 minutes for the booster session. A total of 160 programme sessions (20 groups $x 8$ sessions) were conducted. Only 19 booster sessions were held, rather than the prescribed 20 , because the sessions of two small groups were combined into one. On the registration forms filled in after each session ( $n=178 ; 159$ programme session forms and 19 booster session forms), the facilitators reported carrying out $88 \%(n=156)$ of all sessions according to protocol. Protocol deviations were reported for $12 \%$ of the sessions ( $n=22$, 
i.e. an average of one session for every group). In all cases, this involved skipping, entirely or in part, one of the activities of a session. Since each session consists of a number of different activities, with many activities being repeated in multiple sessions, skipping one activity in a session was considered to be a minor protocol deviation. The facilitators reported no major deviations from protocol.

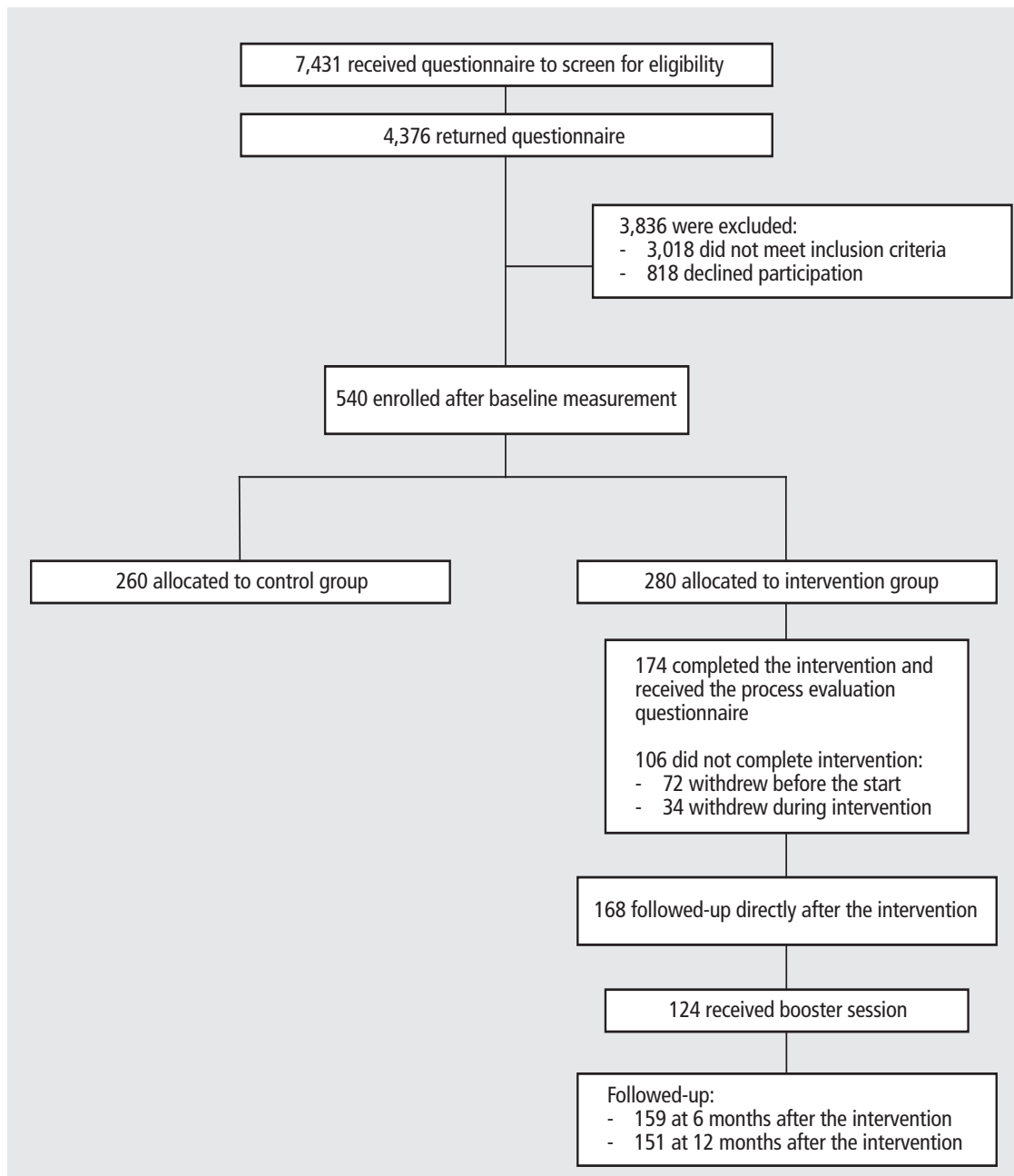

Figure 1 Trial profile - process evaluation 
Table 3 General characteristics of participants

\begin{tabular}{|c|c|c|c|c|}
\hline \multirow{3}{*}{$\begin{array}{l}\text { General characteristics } \\
\text { mean age (standard deviation) }\end{array}$} & \multirow{2}{*}{\multicolumn{2}{|c|}{$\begin{array}{c}\begin{array}{c}\text { Participants who } \\
\text { completed intervention }\end{array} \\
(n=174)\end{array}$}} & \multicolumn{2}{|c|}{$\begin{array}{l}\text { Participants who } \\
\text { withdrew }\end{array}$} \\
\hline & & & & 106) \\
\hline & 77 & (4.5) & 78 & (4.8) \\
\hline number female (\%) & 121 & (70) & 77 & (73) \\
\hline number living alone (\%) & 100 & (58) & 57 & (54) \\
\hline number $\leq$ lower secondary education (\%) & 111 & (64) & 74 & (70) \\
\hline mean cognitive status (standard deviation) ${ }^{*}$ & 32 & (3.5) & 31 & (3.7) \\
\hline number using walking aids (\%) & 61 & (35) & 45 & (43) \\
\hline number fair or poor perceived general health (\%) & 118 & (68) & 79 & (75) \\
\hline number at least one fall in past 6 months (\%) & 96 & (56) & 79 & (57) \\
\hline \multicolumn{5}{|l|}{ Number fear of falling $(\%)^{\dagger}$} \\
\hline sometimes & 108 & (62) & 52 & (49) \\
\hline often & 40 & (23) & 33 & (31) \\
\hline very often & 26 & (15) & 21 & (20) \\
\hline \multicolumn{5}{|l|}{ Number avoidance of activity (\%) ${ }^{\ddagger}$} \\
\hline sometimes & 117 & (67) & 51 & (48) \\
\hline often & 36 & (21) & 40 & (38) \\
\hline very often & 21 & (12) & 15 & (14) \\
\hline
\end{tabular}

\section{Participant attendance}

Of the eight intervention sessions, 162 participants (58\%) attended at least five sessions and 118 participants (42\%) attended less than five sessions. Seventy-two ( $26 \%$ ) of the 280 persons allocated to the intervention group withdrew before the start of the programme. The main reasons for withdrawal were health problems ( $n=25)$, considering the intervention to be inappropriate for their needs $(n=10)$ and being too busy with other activities $(n=10)$. Thirty-four persons $(12 \%)$ withdrew during the programme, after attending an average of 1.6 sessions. The main reasons for withdrawal in this group were health problems $(n=12)$ and finding the intervention to be inappropriate for their needs $(n=8)$.

A total number of 174 persons (62\%) completed the programme, attending 6.8 sessions on average. Thirteen persons from this group withdrew in the period between the end of the programme and the booster session, mostly for reasons related to health $(n=7)$. Of the remaining 161 persons, 124 actually attended the booster session (77\%). On average, 14 persons were allocated to a group. Of these, an average of 10 actually started the programme and nine completed it. An average of six persons participated in the booster session. 


\section{Participant adherence}

Directly after the end of the programme, participants were asked how often they did the homework assigned to them by the facilitator. Of the 167 persons who answered this question, 12 (7\%) reported they did their homework never or rarely, 30 (18\%) that they sometimes did, and 125 (75\%) said they usually or always did their homework. The participants who reported that they sometimes, usually or always did their homework spent an average of 29 minutes (standard deviation $=22$, range $=10-20$ ) on it per session. The facilitators were also asked to assess the adherence to homework for each of their groups. According to the facilitators, in six of the 20 groups the majority of participants did their homework. In eight groups, this applied to about half of the participants, while in six groups only a minority of the participants did their homework. The general quality of the homework made by participants was considered sufficient in 13 groups and insufficient in seven groups.

Directly after the intervention period, the booster session and 6 months after that again, the participants were asked how often they had performed the exercises in the previous period and how much time they had spent performing them. Table 4 shows that the frequency declined considerably in the period between the end of the programme and the 6-month follow-up. However, the exercise frequency remained fairly stable between 6 and 12 months after the programme. Time spent on the exercises also remained stable during the follow-up period. The facilitators were asked to assess, for each of their groups, the adherence to the physical exercises. According to the facilitators, in eight of the 20 groups most participants performed the exercises at home. In seven groups, about half of the participants did the exercises at home, while in five groups only a minority of the participants did so.

Table 4 Adherence to physical exercises according to the participants

\begin{tabular}{|c|c|c|c|}
\hline & $\begin{array}{l}\text { During the programme } \\
\qquad(n=168)\end{array}$ & $\begin{array}{l}\text { After } 6 \text { months } \\
\quad(n=159)\end{array}$ & $\begin{array}{l}\text { After } 12 \text { months } \\
(n=151)\end{array}$ \\
\hline & Number (\%) & Number (\%) & Number (\%) \\
\hline \multicolumn{4}{|c|}{$\begin{array}{l}\text { Frequency of performing physical } \\
\text { exercises }\end{array}$} \\
\hline never & $15 \quad(9)$ & (14) & (15) \\
\hline less than once a week & 13 (8) & (33) & (36) \\
\hline once a week & $40 \quad(24)$ & (25) & (23) \\
\hline more than once a week & $100 \quad(60)$ & (29) & (27) \\
\hline \multicolumn{4}{|c|}{$\begin{array}{l}\text { Average time spent on physical } \\
\text { exercises (per performance)* }\end{array}$} \\
\hline$<10$ minutes & $65 \quad(43)$ & (50) & (47) \\
\hline 10 to 20 minutes & $59 \quad(39)$ & $51 \quad(38)$ & (38) \\
\hline 20 to 30 minutes & $22 \quad(14)$ & (10) & (12) \\
\hline 30 minutes or more & $7 \quad(5)$ & (3) & (3) \\
\hline
\end{tabular}




\section{Opinion of the intervention}

\section{Overall opinion of the intervention and facilitators}

Directly after the programme, participants and facilitators were asked to give the programme a report mark ranging from 1 to 10 , where 1 is the most negative score and 10 the most positive. Participants gave a mean report mark of 8 (range $=5-10, n=168$ ) and facilitators gave a mean report mark of 7.5 (range $=7-8, n=20$ groups assessed by 6 facilitators).

Participants had a very positive opinion of the facilitators; $98 \%$ considered the facilitators to be good or very good. The six facilitators themselves also had a fairly favourable opinion of their own role. In 14 groups, they qualified their functioning as good and in six groups as sufficient.

\section{Benefits reported by participants}

Directly after the programme, participants were asked whether they felt they had benefited from the programme regarding 12 specific topics addressed during the programme (Table 5). The percentage of participants who felt they benefited ranged from $88 \%$ for the topic "I behave more safely" to $46 \%$ for "I avoid fewer activities". In addition, at 6 and 12 months follow-up, participants answered a general question regarding the overall benefit they experienced from the programme in the preceding 6 months. At 6 months follow-up, 71\% of the participants said they had benefited much or very much from the programme in the past 6 months; at 12 months followup, this percentage had fallen somewhat (61\%).

Table 5 Programme benefits according to the participants and the facilitators

\begin{tabular}{lrrrr} 
& $\begin{array}{c}\text { Participants } \\
(\mathbf{n}=174)\end{array}$ & $\begin{array}{c}\text { Facilitators } \\
(\mathbf{n}=6 ; 20\end{array}$ \\
\hline Broups)
\end{tabular}


Facilitators were also asked to assess the benefits of the programme for each of their groups (they were asked "Do you think the participants ..." followed by the items mentioned in the first column of Table 5). In general, the facilitators were less optimistic than participants about the programme benefits. The facilitators were most positive about the benefits in the field of physical activity. They assessed that the majority of participants had become more physically active in 12 of the 20 groups. The influence of the programme on activity restriction was regarded least positively (only 4 of the 20 groups benefited).

\section{Strong and weak aspects of the intervention}

Both participants and facilitators were asked to name strong and weak aspects of the programme (open questions). The strong aspects mentioned most frequently by participants $(n=168)$ were the information provided by the facilitators $(n=65)$, the role of the facilitators (e.g., their enthusiasm and clarity) $(n=63)$, the physical exercises $(n=47)$, the interaction with other participants (e.g., socialising and learning from each other) $(n=43)$, and raising awareness about home safety and safe behaviour $(n=16)$. The strongest aspects of the programme according to the facilitators were the interaction between participants (e.g., showing commitment, learning from each other and helping each other), the physical exercises, raising awareness about home safety and safe behaviour, and the promotion of assertive behaviour.

The weak aspects mentioned most frequently by participants were homework was too much and/or too difficult $(n=25)$, too much repetition of topics ( $n=10)$, the first two sessions were boring $(n=8)$ and too much chattering during the sessions by other participants $(n=6)$. The majority of participants, however, could not come up with any weak aspects $(n=94)$. Weak aspects reported by the facilitators were mainly related to the homework assignments, which were considered too difficult for most participants. According to the facilitators, participants often failed to do their homework as intended. In addition, the facilitators mentioned that participants appeared to have difficulty with abstract thinking (which is needed in the process of cognitive restructuring) and with reproducing the topics discussed in previous sessions.

\section{Suggestions for improvement}

The majority of participants made no suggestions for improvement $(n=91)$. Seventythree participants did have suggestions, these mostly being: simpler homework $(n=19)$, more physical exercises during the sessions $(n=14)$ and additional (booster) sessions $(n=8)$. The main suggestions made by facilitators were simpler homework, a minimum of eight and a maximum of 10 participants per group, fewer topics to be discussed in each session or an increase in the number of sessions, and a more targeted selection of participants. The facilitators considered the intervention especially appropriate for people who feel seriously restricted by their fear of falling, who are motivated to tackle this problem, who are functioning quite well cognitively and who are somewhat used to reading. The facilitators considered the intervention less appropriate for people with strongly impaired vision, a hearing impairment, psychiatric problems like depression, or serious physical impairments. 


\section{Discussion}

In this paper we assessed the feasibility of a cognitive behavioural intervention aimed at reducing fear of falling and associated avoidance of activity among older persons living in the community. As reported by the facilitators there were no major protocol deviations. More than a quarter $(n=72)$ of the 280 participants never started the programme, mainly due to health problems. Of the 208 persons who did start, $84 \%$ completed the programme. The participants reported their adherence as good, but facilitators had a less favourable opinion of this. Both participants and facilitators were positive about the programme and the majority of participants reported benefits from it. Both participants and facilitators provided suggestions for improvement of the intervention.

When comparing our results with the results of Tennstedt and colleagues who originally developed the programme, ${ }^{12}$ the pattern of withdrawals appears to differ. Tennstedt and colleagues reported that $16 \%$ attended no sessions at all, while in our study $26 \%$ attended no sessions at all. The higher percentage of persons who attended no sessions at all in our study may be explained by differences in recruitment strategies and characteristics of the participants. Tennstedt and colleagues recruited participants through self-response to posted notices and individual referrals by housing managers, social workers and case managers, and eligibility of their participants was determined during a home visit. ${ }^{12}$ In our study participants were recruited by means of a short screening questionnaire which was sent to a random sample of older persons. ${ }^{15} \mathrm{~A}$ personal intake procedure as used by Tennstedt and colleagues would probably reduce the percentage of early withdrawal because eligibility criteria can be checked more closely and tailor-made information about the programme can be provided to potential participants. In addition, we included community-living older persons and conducted the intervention at a location somewhere in the vicinity of the participants, while Tennstedt and colleagues recruited persons from senior housing sites and conducted the intervention at these senior housing sites. The latter may have resulted in lower barriers to attend the group meetings.

A possible limitation of our study is the risk that participants may have given socially desirable answers to our questions. We tried to reduce this tendency by using selfadministered questionnaires and by making it clear to participants that the facilitators would not be informed about their individual answers and that their answers would not affect any care needs now or in the future. However, the reasons for withdrawal from the programme were collected by means of telephone interviews, making it perhaps more difficult for participants to report discontent with the programme as the main reason for withdrawal. Strengths of our study are that we collected data from both participants and facilitators by using different methods and that we, in order to avoid bias, analysed the data of the process evaluation before analysing the data of the effect evaluation.

This process evaluation provides insight into the strong and weaker aspects of the intervention. A strong aspect of the intervention is that the majority of participants who actually started the programme, considered it to be beneficial. Another strong aspect is that the six geriatric nurses participating in our study were very capable of conducting the programme and all said that they would continue conducting the programme after the end of the trial, if given the opportunity. The following aspects of the intervention 
need to be improved. First, the homework assignments were found to be too complicated for part of the participants in our research population. Second, participant adherence to the physical exercises seemed not optimal, although restricted adherence to home exercises seems common among community-dwelling older people participating in fall prevention interventions. ${ }^{20} 21$ Third, about a quarter of the selected participants withdrew before the programme even started, mainly because of health problems. This suggests that we did not completely succeed in selecting the most appropriate population, motivating persons who were doubtful about the potential benefits of the programme, and/or meeting the conditions under which even the most frail participants were willing and able to participate (although we offered free transportation to the programme location for those who needed this).

Although we think that some aspects of the intervention could be refined, the results of the present study show that this effective intervention is feasible for both participants and facilitators, and fits in well with regular care. Based on the results of this process evaluation, we recommend adapting the intervention on the following aspects. First, in order to tailor the intervention more to the capacities and skills of the target population, the homework assignments should be simplified to some extent. Second, measures should be taken to increase adherence to the physical exercises. This might be achieved by paying more attention during the programme to factors that may impede the participants from doing the exercises at home and clearly reinforcing the desired behaviour by giving compliments to those participants who show a good adherence. Long-term adherence to the exercises may be improved by adding incentives such as pre-scheduled motivational phone calls. Third, we recommend an individual intake interview for participation in the intervention. In our opinion, such intake interviews should be done by the programme facilitator (a geriatric nurse). Health care professionals, such as general practitioners, community nurses and geriatricians, could refer potential participants to an intake interview. In addition, potential participants could be informed about the programme through announcements in local media. Important factors during the intake interviews include: a) checking whether the potential participant fulfils the eligibility criteria; b) providing clear information about the content of the programme; c) motivating the potential participant; and d) paying attention to factors which may impede participation. We expect that such a procedure would increase the efficiency of the intervention by reducing the number of withdrawals before and during the programme. We recommend implementing the adapted version of the intervention in regular care. For persons with serious health problems for whom it is too burdensome to participate in a group intervention, we recommend developing an individualised in-home version of the intervention.

\section{Acknowledgements}

We would like to thank all participants and facilitators for their participation, and ZonMw - The Netherlands Organisation for Health Research and Development (grant 014-91-052), the Care and Public Health Research Institute, and the Faculty of Health, Medicine and Life Sciences of the Maastricht University for funding this study. 


\section{References}

1. Tinetti ME, Speechley M, Ginter SF. Risk factors for falls among elderly persons living in the community. N Engl J Med 1988;319(26):1701-7.

2. Arfken $\mathrm{CL}$, Lach $\mathrm{HW}$, Birge $\mathrm{SJ}$, et al. The prevalence and correlates of fear of falling in elderly persons living in the community. Am J Public Health 1994;84(4):565-70.

3. Howland J, Lachman ME, Peterson EW, et al. Covariates of fear of falling and associated activity curtailment. Gerontologist 1998;38(5):549-55.

4. Stalenhoef PA, Diederiks JP, Knottnerus JA, et al. The construction of a patient recordbased risk model for recurrent falls among elderly people living in the community. Fam Pract 2000;17(6):490-6.

5. Murphy SL, Williams CS, Gill TM. Characteristics associated with fear of falling and activity restriction in community-living older persons. J Am Geriatr Soc 2002;50(3):516-20.

6. Tinetti ME, Richman D, Powell L. Falls efficacy as a measure of fear of falling. J Gerontol 1990;45(6):P239-43.

7. Howland J, Peterson EW, Levin WC, et al. Fear of falling among the community-dwelling elderly. J Aging Health 1993;5(2):229-43.

8. Tinetti ME, Mendes de Leon CF, Doucette JT, et al. Fear of falling and fall-related efficacy in relationship to functioning among community-living elders. I Gerontol 1994;49(3):M140-7.

9. Fletcher PC, Hirdes JP. Restriction in activity associated with fear of falling among community-based seniors using home care services. Age Ageing 2004;33(3):273-9.

10. Mendes de Leon CF, Seeman TE, Baker DI, et al. Self-efficacy, physical decline, and change in functioning in community-living elders: a prospective study. J Gerontol B Psychol Sci Soc Sci 1996;51(4):S183-90.

11. Cumming RG, Salkeld G, Thomas M, et al. Prospective study of the impact of fear of falling on activities of daily living, SF-36 scores, and nursing home admission. J Gerontol A Biol Sci Med Sci 2000;55(5):M299-305.

12. Tennstedt S, Howland J, Lachman M, et al. A randomized, controlled trial of a group intervention to reduce fear of falling and associated activity restriction in older adults. J Gerontol B Psychol Sci Soc Sci 1998;53(6):P384-92.

13. Tennstedt S, Peterson E, Howland J, et al. 'A Matter of Balance' - Managing Concerns about Falls. Boston: Roybal Center Consortium, Trustees of Boston University, 1998.

14. Zijlstra GA, Tennstedt SL, van Haastregt JC, et al. Reducing fear of falling and avoidance of activity in elderly persons: The development of a Dutch version of an American intervention. Patient Educ Couns 2006;62(2):220-7.

15. Zijlstra GA, van Haastregt JC, van Eijk JT, et al. Evaluating an intervention to reduce fear of falling and associated activity restriction in elderly persons: design of a randomised controlled trial [ISRCTN43792817]. BMC Public Health 2005;5(1):26.

16. Zijlstra GA, van Haastregt JC, van Eijk JT, et al. Prevalence and correlates of fear of falling, and associated avoidance of activity in the general population of community-living older people. Age Ageing 2007;36:304-9.

17. Zijlstra GA, Van Haastregt JC, Ambergen T, et al. Effectiveness of a cognitive behavioural group intervention on fear of falling and associated avoidance of activity in communityliving older people: a randomised controlled trial. Submitted. 
18. Linnan L, Steckler A. Process evaluation for public health interventions and research. An overview. In: Steckler A, Linnan L, editors. Process evaluations for public health interventions and research. San Francisco: Jossey-Bass, 2002:1-23.

19. Oakley $A$, Strange $V$, Bonell $C$, et al. Process evaluation in randomised controlled trials of complex interventions. BMJ 2006;332(7538):413-6.

20. Robertson MC, Devlin N, Gardner MM, et al. Effectiveness and economic evaluation of a nurse delivered home exercise programme to prevent falls. 1: Randomised controlled trial. BMJ 2001;322(7288):697-701.

21. Clemson L, Cumming RG, Kendig $H$, et al. The effectiveness of a community-based program for reducing the incidence of falls in the elderly: a randomized trial. J Am Geriatr Soc 2004;52(9):1487-94. 



\section{CHAPTER 7}

\section{Effectiveness of a cognitive behavioural group intervention on fear of falling and associated avoidance of activity in community-living older people: a randomised controlled trial}




\section{Abstract}

Introduction Fear of falling and associated avoidance of activity are common in older people and may initiate a decline in physical, mental and social health status. We evaluated the effectiveness of a cognitive behavioural group intervention on fear of falling and associated avoidance of activity in community-living older people.

Methods 540 community-living people aged 70 years or older who reported fear of falling and associated avoidance of activity were included in a parallel-group randomised controlled trial. The intervention group $(n=280)$ received an 8-week cognitive behavioural group intervention; the control group $(n=260)$ received no intervention. Data were collected at baseline, and directly, 6 and 12 months after the intervention and were analysed by intention-to-treat using mixed-effects logistic and linear regression.

Results 196 participants of the intervention group and 209 participants of the control group completed the trial. Directly after the intervention, the intervention group benefited regarding fear of falling (odds ratio $=.11 ; 95 \% \mathrm{Cl}=.05-.22 ; \mathrm{p}<.001$ ), avoidance of activity (odds ratio $=.26 ; 95 \% \mathrm{Cl}=.13-.53 ; \mathrm{p}<.001$ ), fall-related selfefficacy (adjusted mean difference $=-1.51 ; 95 \% \mathrm{Cl}=-2.81--.20 ; \mathrm{p}=.02$ ), daily activity (adjusted mean difference $=.95 ; 95 \% \mathrm{Cl}=.22-1.68 ; \mathrm{p}=.01$ ) and several secondary outcomes like anxiety and depression. At 6 and 12 months, various benefits were still present. Compared to the control group, the intervention group reported fewer recurrent falls during the trial.

Conclusion Results of this trial support the effectiveness of a cognitive behavioural group intervention for community-living older people afraid of falling and associated avoidance of activity. 


\section{Introduction}

Falls are common in older people and fall-related injuries are a main public-health concern. ${ }^{1}$ Fear of falling and avoidance of activity due to this fear are associated with falls, but both are common in non-fallers as well. ${ }^{2}$ Of the community-living older population, about $50 \%$ reports fear of falling and about $40 \%$ reports avoidance of activity due to this fear. ${ }^{3}$ There are strong indications that older people who are afraid of falling and consequently avoid activities enter a debilitating spiral of loss of confidence, restriction of physical activities and social participation, physical frailty, falls and loss of independence. ${ }^{45}$ In addition to the adverse consequences on physical, mental and social health status, increased care utilisation and costs may be undesirable consequences as well. ${ }^{4}$ Therefore the importance of strategies to either prevent older people from entering this debilitating spiral or prevent further decline in this spiral is beyond dispute.

A recent systematic review indicated that additional evidence on the effectiveness of community-based fall-related multifactorial group interventions to reduce fear of falling in community-living older people is needed. ${ }^{6}$ In previous studies, cognitive behavioural group interventions showed promising results regarding fear-related outcomes, like anxiety and worry, ${ }^{78}$ and falls self-efficacy, ${ }^{9}$ in older people. In the present paper we report the effectiveness of an 8-week community-based multifactorial, cognitive behavioural group intervention aimed at reducing fear of falling and associated avoidance of activity in community-living older people. We hypothesised that participants of the intervention would benefit regarding our primary outcomes: fear of falling, fall-related self-efficacy, perceived control over falling, avoidance of activity due to fear of falling and daily activity.

\section{Methods}

\section{Study design and participants}

A detailed description of the design of this parallel-group randomised controlled trial (ISRCTN43792817) is published elsewhere. ${ }^{10}$ Between November 2002 and July 2003, questionnaires to screen for eligibility were sent in five consecutive cycles to random selections of the general older population in two communities in The Netherlands. Addresses were obtained from local municipal registry offices. Community-living people aged 70 years or older reporting at least some fear of falling and some avoidance of activity due to fear of falling were included in the trial. People confined to bed, restricted by permanent use of wheelchair, waiting for nursing home admission or participating in other intervention studies were excluded. All participants provided written informed consent. ${ }^{10}$ The Medical Ethics Committee of the Maastricht University/Academic Hospital Maastricht approved the study protocol.

\section{Procedures}

After baseline assessment participants were randomly allocated to the intervention or control group by an independent researcher blinded for participant's characteristics 
using SPSS 12.0. The intervention participants were offered a cognitive behavioural group intervention aimed at reducing fear of falling and associated avoidance of activity. ${ }^{911}$ The control group received no intervention. Participants were not blinded for group allocation.

The cognitive behavioural group intervention comprised 8 weekly sessions of 2 hours and a booster session 6 months after the intervention period. ${ }^{1011}$ To reduce fear of falling and associated avoidance of activity, the intervention aimed to increase selfefficacy beliefs regarding falls, the sense of control over falling, and risk perception and outcomes expectancies regarding falls. Therefore four strategies were applied: (1) restructuring misconceptions to promote a view of fall risk and fear of falling as controllable; (2) setting realistic goals for increasing activity; (3) changing the environment to reduce fall risk; and (4) promoting physical exercise to increase strength and balance. Concisely, after instilling realistic and adaptive views on falls risk and fear of falling, the intervention attempted to accomplish behaviour change. ${ }^{9}$ A variety of techniques and materials were used, including lectures, videos, group discussions, mutual problem solving, assertiveness training and physical exercises for strength, flexibility and balance. Behavioural contracts and goal setting were included to individualise the intervention. Participants received homework, including physical exercises, after each session. ${ }^{9} 10$ The intervention was conducted in local community centres. Free transportation to the intervention site was provided if required. Six trained nurses qualified in geriatric care conducted the intervention; nurses were not blinded for group allocation. In-depth information on the feasibility of the intervention, e.g. performance according to protocol, participant's adherence, are published elsewhere. ${ }^{12}$

At baseline, directly after the intervention, and at 6 and 12 months after the intervention, self-reported data were independently collected using questionnaires and structured telephone interviews. To enhance reliability and validity of the data, we used measures with good psychometric properties and outcome assessors blinded for group allocation were trained to enhance quality of measurement during the interviews. Collected data included primary outcomes: fear of falling, ${ }^{10}$ avoidance of activity due to fear of falling, ${ }^{10}$ fall-related self-efficacy (which assessed fear of falling while performing daily activities), ${ }^{1314}$ perceived control over falling ${ }^{15}$ and daily activity, ${ }^{16}$ and secondary outcomes: perceived general health, ${ }^{17}$ self-rated life satisfaction, ${ }^{18}$ feelings of loneliness, restriction in activities of daily life, ${ }^{19}$ feelings of anxiety, ${ }^{20}$ symptoms of depression, ${ }^{20}$ social support interactions, ${ }^{21}$ perceived consequences of falling - loss of functional independence and damage to identity, ${ }^{2}$ perceived risk of falling and falls. Fall data refer to the number of people sustaining any fall (fallers) and more than one fall (recurrent fallers), number of falls, including indoor and outdoor falls, and number of times fall-related medical attention was required. A fall was defined as an event that results in a person coming to rest inadvertently on the ground or other lower level. ${ }^{22}$ Data on falls were collected by fall calendars for continuous registration; fall calendars were distributed after randomisation. Data on age, gender, living situation, educational level, chronic medical conditions, ${ }^{23}$ cognitive status $^{24}$ and number of falls in the past 6 months were assessed during screening for eligibility or at baseline. 


\section{Statistical analyses}

Sample size calculations based on previous experiences ${ }^{925}$ showed that 92 participants were needed in each group to detect a mean difference of at least 2.5 points on fallrelated self-efficacy based on an alpha of $5 \%$ (two-sided) and power of $80 \%$. Taking a dropout rate of $35 \%$ into account, at least 142 participants were required for each group.

Our analyses were performed according to the intention-to-treat principle. Except for number of falls and number of times fall-related medical attention was required, all outcomes were analysed using mixed-effects logistic or linear regression models. ${ }^{26}$ Regression models accounting for within-person correlation over time using an unstructured covariance structure fitted most outcomes. We examined fixed effects for the interaction group $x$ time to obtain insight into the effectiveness of the intervention at various follow-up moments. Number of falls and fall-related medical attention were analysed using negative binomial regression analysis, which allows dependency between falls within a person and variable follow-up time. ${ }^{27}$ Preplanned per-protocol analyses were performed to compare outcomes of participants who attended at least five sessions of the intervention to outcomes of the control group. Based on prior work, five sessions was considered as sufficient intervention exposure. ${ }^{9}$

We adjusted for possible differences in baseline values and age, gender, living situation, educational level, cognitive status, perceived general health and number of falls in the past 6 months at baseline in all regression analyses. Two-sided significance tests $(p<.05)$ were applied to all analyses. If appropriate, effect sizes were calculated by dividing the difference in mean group scores by the pooled standard deviation. Effect sizes of .20 are considered small, .50 medium and .80 large. ${ }^{28}$

We conducted the negative binomial and mixed-effects logistic regression analyses in STATA version 9.0; all other analyses were performed in SPSS 14.0.

\section{Results}

The study was performed as planned. ${ }^{10}$ Figure 1 shows the number of people screened for eligibility and the progress of participants through the trial. Of the 540 enrolled participants, 280 were randomly allocated to the intervention group and 260 to the control group. During the trial 135 participants were lost to follow-up. Reasons for withdrawal were similar in both groups. Intervention and control group were comparable on most baseline characteristics (Table 1).

In short, the intervention was performed according to protocol and both participants and nurses considered the intervention feasible. ${ }^{12}$ Twenty groups of on average 10 participants received the intervention. Seventy-two participants withdrew before the start of the intervention and 34 participants withdrew during the intervention after attending an average of 1.6 sessions. A total of 162 participants (58\%) attended at least five sessions and 118 participants (42\%) attended less than five sessions. Experiencing health problems was the foremost reason for dropout. Participants stated their adherence to the intervention as good; however, facilitators were less positive about participants' adherence. ${ }^{12}$ 


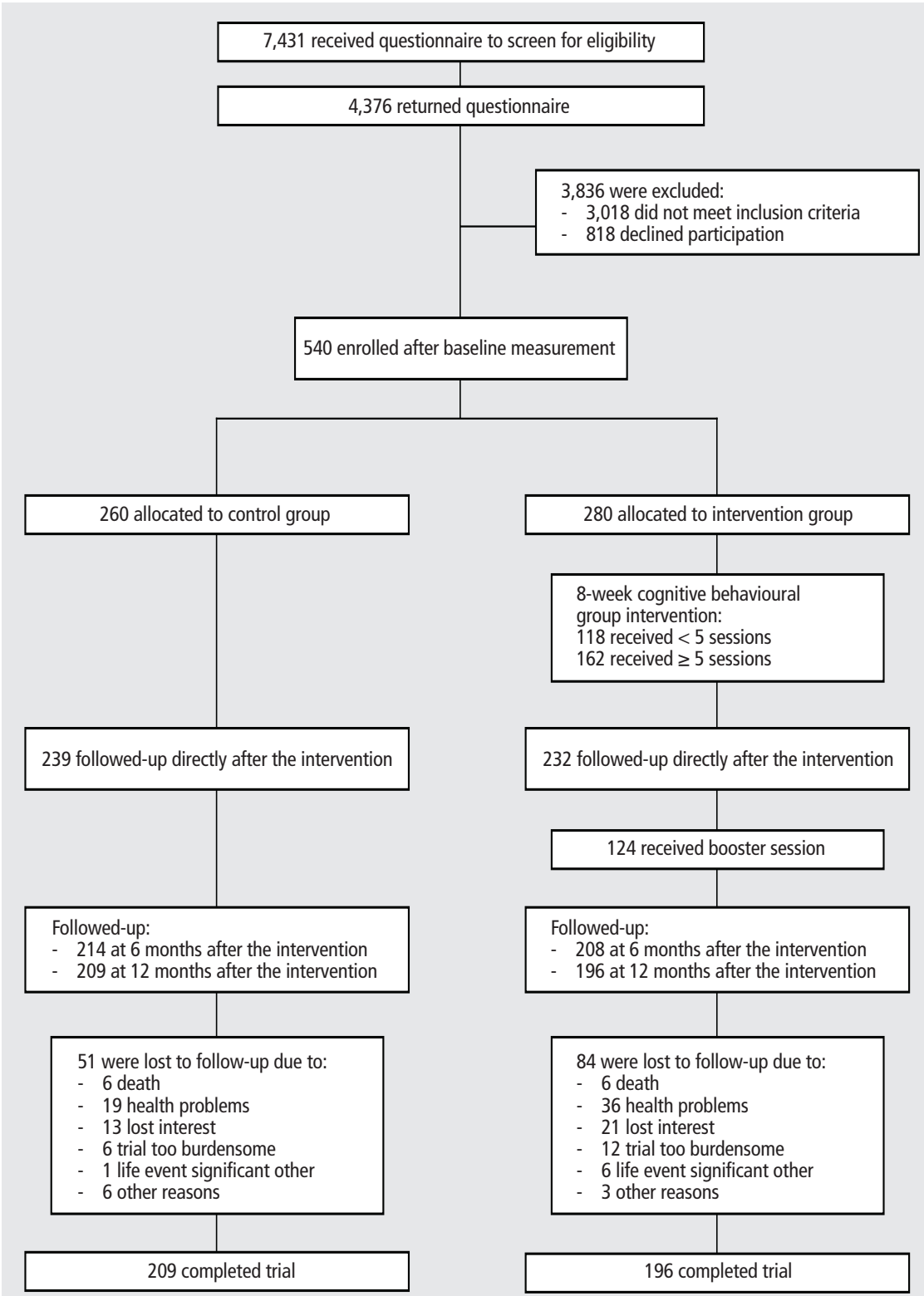

Figure 1 Trial profile - effect evaluation 
Table 1 Baseline characteristics of participants $(N=540)$

\begin{tabular}{|c|c|c|c|c|}
\hline \multirow{2}{*}{$\begin{array}{l}\text { Characteristic*} \\
\text { mean age in years at } 1 \text { January } 2003 \text { (SD) }\end{array}$} & \multicolumn{2}{|c|}{$\begin{array}{l}\text { Control } \\
(n=260)\end{array}$} & \multicolumn{2}{|c|}{$\begin{array}{l}\text { Intervention } \\
(\mathrm{n}=280)\end{array}$} \\
\hline & 78.97 & $(5.0)$ & 77.82 & (4.6) \\
\hline number female (\%) & 190 & (73.1) & 198 & (70.7) \\
\hline number living alone (\%) & 138 & (53.1) & 157 & $(56.1)$ \\
\hline number low educational level $(\%)^{\dagger}$ & 153 & (59.1) & 185 & $(66.1)$ \\
\hline mean cognitive status 0 to $\underline{41}$ (SD) & 32.22 & (3.9) & 31.69 & (3.6) \\
\hline mean number of active diseases (SD) & 2.33 & (1.6) & 2.30 & $(1.5)$ \\
\hline number never fallen in the past 6 months $(\%)^{\dagger}$ & 117 & $(45.0)$ & 123 & $(43.9)$ \\
\hline
\end{tabular}

\section{Primary outcomes}

number fear of falling $(\%)^{\ddagger}$

$\begin{array}{llll}116 & (44.6) \quad 112 & (40.0)\end{array}$

number avoidance of activity due to fear of falling $(\%)^{\ddagger}$

$87 \quad(33.5) \quad 82 \quad(29.3)$

mean fall-related self-efficacy 14 to 56 (SD)

$29.97 \quad(10.2) \quad 28.50 \quad(9.6)$

mean perceived control over falling 4 to $\underline{20}$ (SD)

$13.09 \quad(3.1) \quad 13.48 \quad(3.1)$

mean daily activity 15 to $\underline{60}$ (SD)

$38.16(7.2)$

$39.48 \quad(7.2)$

\begin{tabular}{lllll}
\hline Secondary outcomes & & & & \\
\hline number fair and poor perceived general health $(\%)^{\dagger}$ & 174 & $(66.9)$ & 201 & $(71.8)$ \\
mean self-rated life satisfaction 1 to $\underline{\underline{Z}}$ (SD) & 4.89 & $(1.4)$ & 4.87 & $(1.3)$ \\
mean feelings of loneliness 1 to $\underline{6}$ (SD) & 4.63 & $(1.3)$ & 4.48 & $(1.3)$ \\
mean restriction in activities of daily life $\underline{11}$ to 44 (SD) & 17.40 & $(4.5)$ & 17.14 & $(4.4)$ \\
mean feelings of anxiety $\underline{0}$ to 21 (SD) & 7.55 & $(4.7)$ & 7.17 & $(4.4)$ \\
mean symptoms of depression $\underline{0}$ to 21 (SD) & 6.70 & $(3.9)$ & 6.85 & $(4.0)$ \\
mean social support interactions 12 to $\underline{48}$ (SD) & 30.42 & $(6.8)$ & 28.70 & $(6.6)$ \\
mean CoF - loss of functional independence $\underline{6}$ to 24 (SD) $)^{\S}$ & 14.90 & $(3.4)$ & 14.62 & $(3.4)$ \\
mean CoF - damage to identity $\underline{6}$ to 24 (SD) & 14.89 & $(2.9)$ & 14.73 & $(3.0)$ \\
mean perceived risk of falling $\underline{3}$ to 12 (SD) & 7.23 & $(1.8)$ & 7.12 & $(1.7)$ \\
\hline
\end{tabular}

Note: The underlined score indicates the most favourable score.

* Characteristic presented in number and percentage or mean and standard deviation (SD).

t Low, middle and high educational level; good, fair and poor perceived general health; never, once and more than once fallen.

Often and very often afraid of falling or avoiding activity due to fear of falling.

$\S \mathrm{CoF}=$ perceived consequences of falling.

Table 2 shows the effects on primary and secondary outcomes according to the intention-to-treat analyses. Compared to baseline values, all outcomes improved at all follow-up moments in the intervention group. No adverse events or side effects were reported. Mixed-effects regression analyses showed several statistically significantly improvements in the intervention group compared with the control group. Regarding the primary outcomes, favourable effects were observed for fear of falling, associated avoidance of activity, fall-related self-efficacy and daily activity directly after the intervention; for all outcomes at 6 months; and for fear of falling and perceived control over falling at 12 months after the intervention. Regarding the secondary outcomes, 
favourable effects were observed for feelings of loneliness, restriction of activities of daily life, feelings of anxiety, symptoms of depression, social support interactions and both perceived consequences of falling outcomes directly after the intervention; for restriction of activities of daily life, social support interactions, both perceived consequences of falling outcomes and perceived risk of falling at 6 months; and for social support interactions, perceived damage to identity and perceived risk of falling at 12 months follow-up. Overall, effect sizes were small to medium.

Table 2 Effects of the cognitive behavioural intervention on primary and secondary outcomes $(N=540)^{*}$

\begin{tabular}{|c|c|c|c|c|c|}
\hline & $\begin{array}{l}\text { Control } \\
\text { group }\end{array}$ & $\begin{array}{l}\text { Intervention } \\
\text { group }\end{array}$ & $\begin{array}{c}\text { Adjusted } \\
\text { mixed-effects }\end{array}$ & $\begin{array}{c}\mathrm{p} \\
\text { value }\end{array}$ & $\begin{array}{r}\text { Effec } \\
\text { size }\end{array}$ \\
\hline & Number (\%) & Number (\%) & OR $\quad(95 \% \mathrm{Cl})$ & $p$ & \\
\hline \multicolumn{6}{|l|}{ Primary outcomes } \\
\hline \multicolumn{6}{|l|}{ Fear of falling ${ }^{\dagger}$} \\
\hline direct follow-up & 101 (43.3) & $37(16.3)$ & $.11(.05-.22)$ & .000 & \\
\hline 6-month follow-up & $83(39.0)$ & $51(24.8)$ & $.38(.19-.75)$ & .005 & \\
\hline 12-month follow-up & $86(41.7)$ & $48(24.5)$ & $.31(.15-.61)$ & .001 & \\
\hline \multicolumn{6}{|l|}{$\begin{array}{l}\text { Avoidance of activity } \\
\text { due to fear of falling }{ }^{\dagger}\end{array}$} \\
\hline direct follow-up & $71(30.5)$ & 35 (15.4) & $.26(.13-.53)$ & .000 & \\
\hline 6-month follow-up & 79 (37.1) & $45 \quad(22.0)$ & $.34(.18-.67)$ & .002 & \\
\hline \multirow[t]{3}{*}{ 12-month follow-up } & $71 \quad(34.5)$ & $50(25.5)$ & $.54(.28-1.05)$ & .07 & \\
\hline & & & Mean & & \\
\hline & Mean (SD) & Mean (SD) & difference $(95 \% \mathrm{Cl})$ & $p$ & $d$ \\
\hline \multicolumn{6}{|l|}{$\begin{array}{l}\text { Fall-related self-efficacy } \\
\text { (14 to 56) }\end{array}$} \\
\hline direct follow-up & $28.25(10.8)$ & $25.47(9.7)$ & $-1.51 \quad(-2.81--.20)$ & .02 & .27 \\
\hline 6-month follow-up & $29.36(11.0)$ & $25.13(9.7)$ & $-2.61(-4.22--.99)$ & .002 & .41 \\
\hline 12-month follow-up & $28.86(11.0)$ & $26.26(10.9)$ & $-1.50(-3.14-.13)$ & .07 & \\
\hline
\end{tabular}

Perceived control over falling

(4 to $\underline{20}$ )

$\begin{array}{lllllllll}\text { direct follow-up } & 13.88 & (2.9) & 14.40 & (2.8) & .46 & (-.02-.92) & .06 & - \\ \text { 6-month follow-up } & 13.57(3.0) & 14.28 & (2.7) & .73(.23-1.24) & .005 & .25 \\ \text { 12-month follow-up } & 13.46(3.1) & 14.42 & (2.9) & & .90(.38-1.43) & .001 & .32\end{array}$

Daily activity

(15 to $\underline{60)}$

\begin{tabular}{lllllllc} 
direct follow-up & $37.71(7.7)$ & $40.09(6.5)$ & $.95(.22-1.68)$ & .01 & .33 \\
6-month follow-up & $37.97(7.4)$ & $40.29(6.9)$ & $.94(.13-1.74)$ & .02 & .33 \\
12-month follow-up & $37.68(7.6)$ & $39.65(7.4)$ & $.54(-.35-1.42)$ & .24 & - \\
\hline
\end{tabular}


Table 2 Effects of the cognitive behavioural intervention on primary and secondary outcomes $(\mathrm{N}=540)^{\text {* }}$ (continued)

\begin{tabular}{|c|c|c|c|c|c|c|}
\hline & $\begin{array}{l}\text { Control } \\
\text { group }\end{array}$ & $\begin{array}{l}\text { Intervention } \\
\text { group }\end{array}$ & & $\begin{array}{l}\text { Adjusted } \\
\text { xed-effects }\end{array}$ & $\begin{array}{c}\mathrm{p} \\
\text { value }\end{array}$ & $\begin{array}{r}\text { Effect } \\
\text { size }\end{array}$ \\
\hline & Number (\%) & Number (\%) & & $(95 \% \mathrm{Cl})$ & $p$ & \\
\hline \multicolumn{7}{|l|}{ Secondary outcomes } \\
\hline \multicolumn{7}{|l|}{$\begin{array}{l}\text { Perceived } \\
\text { general health }\end{array}$} \\
\hline direct follow-up & $151(64.8)$ & $144(63.4)$ & .73 & $3(.38-1.38)$ & .33 & \\
\hline 6-month follow-up & $128(60.1)$ & $133(64.6)$ & 1.24 & $(.63-2.42)$ & .53 & \\
\hline \multirow[t]{2}{*}{ 12-month follow-up } & $123(59.7)$ & 131 (66.8) & 1.63 & $(.82-3.24)$ & .16 & \\
\hline & Mean (SD) & Mean (SD) & $\begin{array}{r}\text { Mean } \\
\text { difference }\end{array}$ & $(95 \% \mathrm{Cl})$ & $p$ & $d$ \\
\hline \multicolumn{7}{|c|}{$\begin{array}{l}\text { Self-rated life satisfaction } \\
\text { (1 to } \underline{7})\end{array}$} \\
\hline direct follow-up & $5.05(1.5)$ & $5.15(1.3)$ & .10 & $(-.12-.32)$ & .37 & - \\
\hline 6-month follow-up & $5.04(1.3)$ & $5.10(1.4)$ & .07 & $7(-.17-.30)$ & .58 & - \\
\hline 12-month follow-up & $4.89(1.5)$ & $4.89(1.5)$ & .01 & $(-.27-.29)$ & .97 & - \\
\hline \multicolumn{7}{|l|}{$\begin{array}{l}\text { Feelings of loneliness } \\
\text { (1 to } \underline{6})\end{array}$} \\
\hline direct follow-up & $4.56(1.3)$ & $4.66(1.2)$ & .16 & $5(.001-.33)$ & .05 & .07 \\
\hline 6-month follow-up & $4.57(1.3)$ & $4.66(1.2)$ & .16 & $5(-.003-.33)$ & .06 & - \\
\hline 12-month follow-up & $4.54(1.2)$ & $4.61(1.3)$ & .15 & $5(-.05-.35)$ & .14 & - \\
\hline \multicolumn{7}{|c|}{$\begin{array}{l}\text { Restriction in activities of daily } \\
\text { life (11 to 44) }\end{array}$} \\
\hline direct follow-up & $17.55(4.7)$ & $16.72(4.7)$ & -.52 & $(-1.03--.004)$ & .05 & .18 \\
\hline 6-month follow-up & $18.18(5.0)$ & $17.05(4.8)$ & -.78 & $3(-1.45--.10)$ & .02 & .23 \\
\hline 12-month follow-up & $17.54(5.0)$ & $16.81(4.8)$ & -.60 & $(-1.24-.05)$ & .07 & - \\
\hline \multicolumn{7}{|l|}{$\begin{array}{l}\text { Feelings of anxiety } \\
(\underline{0} \text { to } 21)\end{array}$} \\
\hline direct follow-up & $7.08(4.7)$ & $6.29(4.1)$ & -.55 & $(-1.10--.000)$ & .05 & .18 \\
\hline 6-month follow-up & $7.42(4.7)$ & $6.60(4.4)$ & -.53 & $3(-1.15-.10)$ & .10 & - \\
\hline 12-month follow-up & $7.14(4.6)$ & $6.46(4.5)$ & -.39 & $9(-1.04-.26)$ & .24 & - \\
\hline \multicolumn{7}{|l|}{$\begin{array}{l}\text { Symptoms of depression } \\
(0 \text { to } 21)\end{array}$} \\
\hline direct follow-up & $6.56(4.2)$ & $5.86(3.8)$ & -.66 & $5(-1.15--.18)$ & .007 & .18 \\
\hline 6-month follow-up & $6.46(4.2)$ & $6.10(4.0)$ & -.37 & $7(-.92-.18)$ & .19 & - \\
\hline 12-month follow-up & $6.50(3.9)$ & $6.15(4.0)$ & -.33 & $3(-.90-.24)$ & .25 & - \\
\hline
\end{tabular}


Table 2 Effects of the cognitive behavioural intervention on primary and secondary outcomes ( $N=540)^{*}$ (continued)

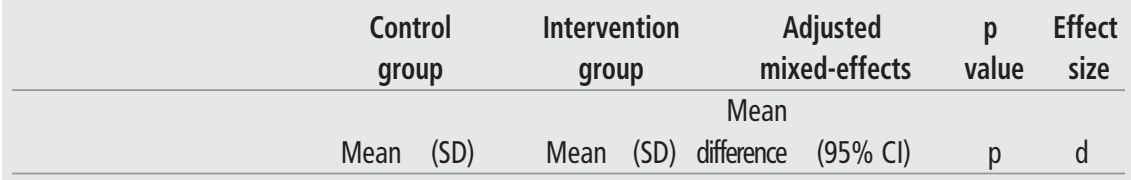

Social support interactions

(12 to $\underline{48})$

$\begin{array}{lllllllll}\text { direct follow-up } & 29.46 & (7.2) & 29.99 & (6.1) & 1.47 & (.60-2.34) & .001 & .08 \\ \text { 6-month follow-up } & 30.09 & (7.4) & 30.44 & (6.7) & 1.43 & (.50-2.37) & .003 & .05 \\ \text { 12-month follow-up } & 29.70 & (7.5) & 29.96 & (6.6) & 1.35 & (.35-2.35) & .009 & .04\end{array}$

CoF - loss of functional

independence ( $\underline{6}$ to 24$)^{\S}$

$\begin{array}{lrlllllll}\text { direct follow-up } & 15.15 & (3.4) & 13.82 & (3.1) & -1.26 & (-1.80--.73) & .000 & .41 \\ \text { 6-month follow-up } & 15.32 & (3.5) & 14.14 & (3.2) & -.88 & (-1.47--.30) & .003 & .35 \\ \text { 12-month follow-up } & 14.84 & (3.4) & 14.09 & (3.0) & -.44 & (-1.02-.15) & .14 & -\end{array}$

CoF - damage to identity

$(\underline{6} \text { to } 24)^{\S}$

$\begin{array}{lllllllll}\text { direct follow-up } & 15.19 & (2.5) & 14.39 & (2.6) & -.72 & (-1.13--.30) & .001 & .32 \\ \text { 6-month follow-up } & 15.03 & (2.4) & 14.40 & (2.7) & -.52 & (-.96--.09) & .02 & .25 \\ \text { 12-month follow-up } & 15.07 & (2.5) & 14.32 & (2.7) & -.63 & (-1.07--.19) & .006 & .29\end{array}$

Perceived risk of falling

(3 to 12$)$

\begin{tabular}{|c|c|c|c|c|c|c|c|c|}
\hline direct follow-up & 7.41 & (1.7) & 7.08 & (1.5) & -.27 & $(-.58-.04)$ & .09 & - \\
\hline 6-month follow-up & 7.52 & (1.7) & 6.99 & (1.4) & -.44 & $(-.73--.15)$ & .003 & .35 \\
\hline 12-month follow-up & 7.50 & (1.5) & 7.00 & (1.5) & -.44 & $(-.75--.14)$ & .004 & .33 \\
\hline
\end{tabular}

Note: The underlined score indicates the most favourable score.

* Mixed-effects logistic and linear regression models adjusted for baseline values and age, gender, living situation, educational level, cognitive status, perceived general health and number of falls in the past 6 months. $O R=$ odds ratio obtained by mixed-effects logistic regression; $95 \% \mathrm{Cl}=95 \%$ confidence interval; $\mathrm{SD}=$ standard deviation.

${ }^{\dagger}$ Often and very often afraid of falling or avoiding activity due to fear of falling.

₹ Fair and poor preceived general health.

$\S \mathrm{COF}=$ perceived consequences of falling.

Table 3 presents the outcomes of the intention-to-treat analyses on the fall data. Both the number of fallers and recurrent fallers increased more over time in the control group compared with the intervention group. Fall data from baseline until 12 months after the intervention showed statistically significant less recurrent fallers in the intervention group. No statistically significant differences were found for number of falls during the trial, indoor and outdoor, and number of times fall-related medical attention was required.

Compared to the intention-to-treat outcomes, the per-protocol analyses (data not 
Table 3 Effects of a cognitive behavioural intervention on falls $(N=540)$

\begin{tabular}{|c|c|c|c|c|c|}
\hline & $\begin{array}{l}\text { Control } \\
\text { group }\end{array}$ & $\begin{array}{l}\text { Intervention } \\
\text { group }\end{array}$ & $\begin{array}{r}\text { Mixed- } \\
\text { an } \\
\text { binom }\end{array}$ & $\begin{array}{l}\text { effects logistic } \\
\text { d negative } \\
\text { ial regression* }\end{array}$ & $p$ value \\
\hline & Number (\%) & Number (\%) & OR & $(95 \% \mathrm{Cl})$ & $p$ \\
\hline \multicolumn{6}{|l|}{ Fallers $^{\dagger}$} \\
\hline baseline until direct follow-up & $50(21.2)$ & $46(20.4)$ & .96 & $(.43-2.16)$ & .92 \\
\hline baseline until 6-month follow-up & $95(44.6)$ & $80(40.0)$ & .74 & $(.35-1.60)$ & .47 \\
\hline baseline until 12-month follow-up & $117(57.6)$ & $91(48.4)$ & .50 & $(.23-1.08)$ & .08 \\
\hline \multicolumn{6}{|l|}{ Recurrent fallers ${ }^{\dagger}$} \\
\hline baseline until direct follow-up & $16(6.8)$ & $13(5.8)$ & .80 & $(.25-2.53)$ & .71 \\
\hline baseline until 6-month follow-up & $53(24.9)$ & $35(17.5)$ & .48 & $(.20-1.12)$ & .09 \\
\hline \multirow[t]{2}{*}{ baseline until 12-month follow-up } & $76(37.4)$ & $48(25.5)$ & .38 & $(.17-.84)$ & .02 \\
\hline & Number & Number & IRR & $(95 \% \mathrm{Cl})$ & p \\
\hline Falls during trial $^{\dagger}$ & 381 & 302 & .86 & $(.65-1.14)$ & .28 \\
\hline indoor falls during trial & 224 & 198 & .90 & $(.64-1.27)$ & .54 \\
\hline outdoor falls during trial & 157 & 104 & .77 & $(.55-1.09)$ & .14 \\
\hline Fall-related medical attention ${ }^{\dagger}$ & 102 & 75 & .78 & $(.45-1.34)$ & .36 \\
\hline
\end{tabular}

shown) showed additional favourable effects for perceived control over falling and perceived risk of falling at direct follow-up and avoidance of activity, fall-related selfefficacy, daily activity, feelings of loneliness and feelings of anxiety at 12 months followup. Fall data showed additional favourable effects for number of fallers until 12 months follow-up and number of times fall-related medical attention was required. Total number of falls, number of indoor falls and number of outdoor falls approached the level of significant difference.

\section{Discussion}

This randomised controlled trial showed that an 8-week cognitive behavioural group intervention improved fear of falling, associated avoidance of activity, fall-related selfefficacy, perceived control over falling and daily activity in community-living older people. Favourable effects were also observed in a broad range of other psychosocial and physical outcomes, like loneliness, restriction of activities of daily life, anxiety, depression, social support and recurrent falls. Generally, small to medium effects were present until 6 months after the intervention.

In contrast to many interventions designed to reduce falls and risk of falls, ${ }^{29}$ only a 
few interventions have explicitly tried to improve psychological outcomes related to falling, such as fear of falling, in community-living older people. In two trials, evaluating an intense tai chi chuan intervention ${ }^{30}$ and a cognitive behavioural intervention, ${ }^{9}$ benefits were reported directly after the intervention regarding confidence in performing activities without falling. In the cognitive behavioural intervention these benefits were solely observed in participants who complied with the intervention. Contrary to the tai chi chuan intervention, long-term effects, at 6 and 12 months, were studied in the cognitive behavioural intervention. Only at 12 months benefits regarding confidence and mobility were observed in compliers with the intervention. ${ }^{9}$ In another study, a multifaceted intervention yielded benefits in mobility self-efficacy after one year, however, not in confidence in performing activities without falling and physical activity. ${ }^{31}$

Compared to these studies, our cognitive behavioural intervention has several strengths. First, favourable effects for fear of falling and for daily activity are shown through at least 6 months after the intervention. Second, our intervention showed unambiguous improvements on a variety of other psychosocial and physical outcomes, like anxiety, depression, social interaction and recurrent falls. Third, improved fallrelated feelings of control and perceptions of risk and consequences support the effectiveness of our applied intervention strategies, i.e. cognitive restructuring, setting realistic goals for behaviour change, skills mastery and modelling. As previously hypothesised, ${ }^{32}$ our study confirmed that an intervention explicitly aimed at reducing fear of falling can influence outcome expectancies like perceived consequences of falling. Like self-efficacy beliefs, outcome expectancies are considered important predictors of behaviour change. ${ }^{33} 34$ Behaviour change like increased activity, however, may lead to increased fall risk due to greater exposure. ${ }^{35}$ Yet in our study, regardless of increased daily activity in the intervention group, fewer falls were experienced by the intervention group over the course of the trial period. This may underscore both the protective effect of daily activity in falls prevention and the effect of realistic and adaptive views on falls risk and fear of falling as important precursors for safe behaviour.

Generally, our effect sizes were small to medium and some effects decayed over time. To attain long-term benefits our intervention may be enhanced by improved intervention uptake and adherence to intervention sessions as $42 \%$ of our intervention group withdrew before the start of the intervention or attended less than five sessions. Problems with uptake and adherence are common in fall-related interventions and improvements in participation are expected if health care professionals provide encouragement. ${ }^{36}$ Therefore it is important that these professionals, for instance general practitioners, identify fearful and avoidant older people, refer them to relevant interventions and show interest regarding intervention participation in future encounters. Furthermore, an in-person recruitment strategy in which potential participants are screened for eligibility and receive additional trial information by a facilitator or research assistant ${ }^{9}{ }^{31}$ might improve uptake as well.

In addition, improved adherence to homework and prolongation of intervention duration might also enhance long-term benefit as both are important features for effective interventions in older people due to reduced learning and memory abilities in this population. ${ }^{37}$ Compared to the small benefits in compliers with the cognitive behavioural intervention as shown by Tennstedt and colleagues, ${ }^{9}$ our intervention 
showed benefits in the intervention group as randomised and subsequently additional effects in those who attended five or more sessions. Adaptations to the intervention protocol, ${ }^{11}$ like weekly sessions and a booster session, might have contributed to the benefits shown by our intervention. However, improved adherence to homework, for instance by providing personal feedback, and adding regular or booster sessions to our intervention might result in stronger effects and prolonged benefits.

We acknowledge several factors that may be important considering the outcomes of our study. First, blinding of participants and facilitators is highly complicated in many intervention studies ${ }^{38}$ and in our study participants and facilitators were not blinded for group allocation. Accordingly our control group received no placebo intervention that controlled for contact time and the effect of attention may have contributed to our positive outcomes. This may have caused larger estimates of effects. Social elements and attention, i.e. modelling and mutual problem solving, however, can be considered essential components of our group intervention. Second, despite efforts to enhance data completeness, study dropouts resulted in missing data. Study dropouts were more likely to be frailer, both physically and mentally, at baseline. However, by applying mixed-effect regression techniques, all available data of participants were included in the analyses. Next, results of our per-protocol analyses should be interpreted with care. Although we adjusted for several variables in the analyses, the advantages of randomisation might no longer apply due to selection bias. Lastly, generalisation of our results to populations with other characteristics, like poorer cognitive or physical functioning, may not be appropriate.

Several recommendations for research and practice stem from this study. For future research, studies aiming to reduce fear of falling and associated avoidance of activity may focus on obtaining larger effects and prolonged intervention benefits on multiple outcomes. This might involve research on optimising intervention characteristics, like frequency and duration of sessions, and adherence to homework. Additionally, considering the variety of intervention effects and the limited uptake and adherence, a substantial group of older people that might benefit considerably from reduced fear falling and increased activity is not reached. For housebound older people with potentially higher risk of falls and fall-related injuries or people not interested in group interventions, the development and evaluation of an in-home cognitive behavioural intervention might be appropriate. Moreover, our findings lend support to the relationship between psychological outcomes of falling and actual falls and confirm the importance of including these kinds of outcomes both in fall-related interventions and clinical assessments of falls risk. For practice, the positive results of this trial support implementation of a cognitive behavioural group intervention for community-living older people afraid of falling and associated avoidance of activity in regular care. To improve intervention participation and herewith the participant's benefit of the intervention, health care professionals should be involved in encouraging potential participants to attend appropriate and effective fall-related prevention strategies.

In conclusion, this cognitive behavioural intervention showed to have positive and lasting effects on both fear of falling and associated avoidance of activity as well as several other outcomes in community-living older people. The study provided evidence to suggest that reducing fear of falling also has an effect on rate of falls. 


\section{Acknowledgements}

We kindly thank all participants and nurses for their numerous efforts that enabled evaluating the Dutch cognitive behavioural intervention (AMB-NL). The Centre for Data and Information Management (MEMIC) and Vonca Schaffers are acknowledged for their assistance in the data collection, and Hans Bosma for randomisation of participants. ZonMw - The Netherlands Organisation for Health Research and Development (grant 014-91-052), the School for Public Health and Primary Care (Caphri), and the Faculty of Health, Medicine and Life Sciences of the Maastricht University funded this study. 


\section{References}

1. Kannus $P$, Sievanen $H$, Palvanen $M$, et al. Prevention of falls and consequent injuries in elderly people. Lancet 2005;366(9500):1885-93.

2. Yardley L, Smith H. A prospective study of the relationship between feared consequences of falling and avoidance of activity in community-living older people. Gerontologist 2002;42(1):17-23.

3. Zijlstra GA, van Haastregt JC, van Eijk JT, et al. Prevalence and correlates of fear of falling and associated avoidance of activity in the general population of community-living older people. Age Ageing;36:304-9.

4. Cumming RG, Salkeld G, Thomas M, et al. Prospective study of the impact of fear of falling on activities of daily living, SF-36 scores, and nursing home admission. J Gerontol A Biol Sci Med Sci 2000;55(5):M299-305.

5. Delbaere K, Crombez G, Vanderstraeten G, et al. Fear-related avoidance of activities, falls and physical frailty. A prospective community-based cohort study. Age Ageing 2004;33(4):368-73.

6. Zijlstra GA, van Haastregt JC, van Rossum E, et al. Interventions to reduce fear of falling in community-living older people: a systematic review. J Am Geriatr Soc 2007;55:603-15.

7. Stanley MA, Beck JG, Novy DM, et al. Cognitive-behavioral treatment of late-life generalized anxiety disorder. J Consult Clin Psychol 2003;71(2):309-19.

8. Wetherell JL, Gatz M, Craske MG. Treatment of generalized anxiety disorder in older adults. J Consult Clin Psychol 2003;71(1):31-40.

9. Tennstedt S, Howland J, Lachman M, et al. A randomized, controlled trial of a group intervention to reduce fear of falling and associated activity restriction in older adults. J Gerontol B Psychol Sci Soc Sci 1998;53(6):P384-92.

10. Zijlstra GA, van Haastregt JC, van Eijk JT, et al. Evaluating an intervention to reduce fear of falling and associated activity restriction in elderly persons: design of a randomised controlled trial [ISRCTN43792817]. BMC Public Health 2005;5(1):26.

11. Zijlstra GA, Tennstedt SL, van Haastregt JC, et al. Reducing fear of falling and avoidance of activity in elderly persons: The development of a Dutch version of an American intervention. Patient Educ Couns 2006;62(2):220-7.

12. van Haastregt JCM, Zijlstra GAR, van Rossum E, et al. Feasibility of a cognitive behavioural group intervention to reduce fear of falling and associated avoidance of activity in elderly people living in the community. BMC Health Serv Res 2007;7:156.

13. Tinetti ME, Mendes de Leon CF, Doucette JT, et al. Fear of falling and fall-related efficacy in relationship to functioning among community-living elders. J Gerontol 1994;49(3):M140-7.

14. Hill KD, Schwarz JA, Kalogeropoulos AJ, et al. Fear of falling revisited. Arch Phys Med Rehabil 1996;77(10):1025-9.

15. Lawrence RH, Tennstedt SL, Kasten LE, et al. Intensity and correlates of fear of falling and hurting oneself in the next year: baseline findings from a Roybal Center fear of falling intervention. J Aging Health 1998;10(3):267-86.

16. Holbrook M, Skilbeck CE. An activities index for use with stroke patients. Age Ageing 1983;12(2):166-70. 
17. Stewart AL, Hays RD, Ware JE, Jr. The MOS short-form general health survey. Reliability and validity in a patient population. Med Care 1988;26(7):724-35.

18. Andrews FM, Whitney SB. Social indicators of well-being. New York: Plenum Press, 1976.

19. Kempen Gl, Miedema I, Ormel J, et al. The assessment of disability with the Groningen Activity Restriction Scale: Conceptual framework and psychometric properties. Soc Sci Med 1996;43(11):1601-10.

20. Bjelland I, Dahl AA, Haug TT, et al. The validity of the Hospital Anxiety and Depression Scale. An updated literature review. J Psychosom Res 2002;52(2):69-77.

21. Kempen GI, Van Eijk LM. The psychometric properties of the SSL12-I, a short scale for measuring social support in the elderly. Soc Indic Res 1995;35(3):303-12.

22. The prevention of falls in later life. A report of the Kellogg International Work Group on the prevention of falls by the elderly. Dan Med Bull 1987;47(12):1397-402.

23. CBS. Gezondheidsenquete 1989 (Health Interview Survey). Voorburg/Heerlen, The Netherlands, 1989.

24. Brandt J, Spencer M, Folstein M. The Telephone Interview for Cognitive Status. Neuropsychiatry Neuropsychol Behav Neurol 1988;1(2):111-7.

25. van Haastregt JC, Diederiks JP, van Rossum E, et al. Effects of a programme of multifactorial home visits on falls and mobility impairments in elderly people at risk: randomised controlled trial. BMJ 2000;321(7267):994-8.

26. Verbeke G, Molenberghs G. Linear mixed models for longitudinal data. 1st ed. New York: Springer-Verlag, 2000.

27. Robertson MC, Campbell AJ, Herbison P. Statistical analysis of efficacy in falls prevention trials. J Gerontol A Biol Sci Med Sci 2005;60(4):530-4.

28. Cohen J. A power primer. Psychol Bull 1992;112(1):155-9.

29. Gillespie LD, Gillespie WJ, Robertson MC, et al. Interventions for preventing falls in elderly people. Cochrane Database Syst Rev 2003(4):CD000340.

30. Zhang JG, Ishikawa-Takata K, Yamazaki H, et al. The effects of Tai Chi Chuan on physiological function and fear of falling in the less robust elderly: an intervention study for preventing falls. Arch Gerontol Geriatr 2006;42(2):107-16.

31. Clemson L, Cumming RG, Kendig H, et al. The effectiveness of a community-based program for reducing the incidence of falls in the elderly: a randomized trial. J Am Geriatr Soc 2004;52(9):1487-94.

32. Yardley L, Kempen GI. Measuring expected outcomes of falls. J Am Geriatr Soc 2006;54(8):1300-1.

33. Bandura A. Self-efficacy. The exercise of control. New York: W.H. Freeman, 1997.

34. Lach HW. Self-efficacy and fear of falling: in search of complete theory. J Am Geriatr Soc 2006;54(2):381-2.

35. Rubenstein LZ, Josephson KR, Trueblood PR, et al. Effects of a group exercise program on strength, mobility, and falls among fall-prone elderly men. J Gerontol A Bio/ Sci Med Sci 2000;55(6):M317-21.

36. Yardley L, Bishop FL, Beyer N, et al. Older people's views of falls-prevention interventions in six European countries. Gerontologist 2006;46(5):650-60.

37. Satre DD, Knight BG, David S. Cognitive-behavioural interventions with older adults: integrating clinicial and gerontological research. Prof Psychol Res Pr 2006;37(5):489-98. 
38. Schulz KF, Chalmers I, Hayes RJ, et al. Empirical evidence of bias. Dimensions of methodological quality associated with estimates of treatment effects in controlled trials. JAMA 1995;273(5):408-12. 



\section{CHAPTER 8}

\section{Mediating effects of psychosocial factors on fear of falling and daily activity in a cognitive behavioural intervention}




\section{Abstract}

Objective This study explored the mediating effects of psychosocial factors on trajectories of fear of falling and daily activity in a cognitive behavioural intervention.

Methods Our study sample comprised 540 community-living older people who participated in a randomised controlled trial evaluating this intervention. Control beliefs, self-efficacy beliefs, outcome expectations and social interactions, as potential mediators, and fear of falling and daily activity, as outcome variables, were assessed before, directly after, and at 6 and 12 months after the intervention.

Results The mixed-effects regression analyses showed small to moderate statistically significant intervention effects on the potential mediators at nearly all follow-up assessments and modest mediating effects of the separate psychosocial factors on the outcomes.

Conclusion When all mediators were taken into account, a substantial amount of the association between the intervention and the outcomes was explained. 


\section{Introduction}

Concerns about falls are common in older populations. Of the community-living older people approximately 50\% reports fall-related fear ${ }^{12}$ and approximately $40 \%$ reports avoidance of activity due to this fear. ${ }^{2-4}$ In addition to recent fallers, older people who have not recently fallen frequently report fear of falling and associated activity restriction as well. ${ }^{34}$ In older populations, avoiding activities due to fear of falling may be regarded as an attempt to minimise the risk and potential consequences of falling. However, excessive fear of falling and inappropriate responses to this fear, such as unnecessary activity restriction, may lead to reduced physical, psychological and social functioning. This is reflected in several studies that showed that fear of falling is associated with adverse effects such as decreased physical activity, quality of life, life satisfaction and social participation, and increased risk of falls and institutionalisation. ${ }^{3-7}$ Obviously, these adverse effects lead to increased health care utilisation ${ }^{7}$ and consequently to increased public expenditure. This suggests that, except for physical trauma immediately caused by a fall, the consequences of excessive fear of falling and unnecessary restriction of activities may be just as harmful as a fall itself. ${ }^{7}$ Therefore, effective strategies to prevent or reduce this fear, promote safe behaviour and increase daily activity are important.

Effective interventions reducing fall-related fear and increasing levels of activity in community-living older people are scarce. Several interventions showed to reduce fear of falling or to improve confidence regarding performing activities without falling. ${ }^{8}$ However, benefits of these interventions regarding performed daily, physical or social activity were rarely reported. Two cognitive behavioural group interventions explicitly aimed at reducing excessive fall-related fear and unnecessary avoidance of activity showed beneficial outcomes in randomised controlled trials in community-living older people. ${ }^{910}$ In the first study confidence in performing activities without falling and mobility were improved directly and 12 months after a cognitive behavioural intervention in compliant participants. ${ }^{9}$ These encouraging findings were reinforced by a recent study evaluating an adapted version of this cognitive behavioural intervention. This study showed benefits regarding fear of falling, associated avoidance of activity and daily activity till 6 months after the intervention in the intervention group as randomised and till 12 months after the intervention in compliant participants. ${ }^{10}$ Regardless of these beneficial outcomes further study is warranted, as intervention effects in both trials were generally small to medium. Insight into the mechanism underlying the cognitive behavioural intervention effects may facilitate intervention improvement and may consequently lead to larger effects. ${ }^{11}$

In the current study we explored potential psychosocial mediators in the association between the aforementioned effective cognitive behavioural group intervention ${ }^{10}$ and fear of falling and daily activity. This intervention is primarily based on the social cognitive theory ${ }^{12}$ and attempts to obtain intervention effects by improving control and self-efficacy beliefs. ${ }^{913}$ Control beliefs reflect "the extent to which one regards one's life-chances as being under one's own control in contrast to being fatalistically ruled". ${ }^{14}$ The concept of self-efficacy is derived from the social cognitive theory that supposes that people exercise control over motivation and behaviour and supports a transactional view of self and society in which personal factors, environmental factors and behaviour operate as 
reciprocally, interacting factors. ${ }^{12}$ Within this theory, self-efficacy beliefs, that is "a judgment of one's ability to organise and execute given types of performances", and outcome expectations, that is "a judgment of the likely consequences such performances will produce", are considered important determinants of behaviour, which are shaped by skills mastery, modelling, verbal persuasion and experiencing physiological states. ${ }^{15} 16$ Obviously, social interactions play a vital role in this theory because behaviour and social systems are interwoven and modelling and verbal persuasion, for which social interactions are necessary, are important determinants for behavior. ${ }^{15}$ Improvements in control beliefs, self-efficacy beliefs, outcome expectations and social interactions may facilitate coping with fear of falling and activities in daily life. Therefore, we hypothesised that control beliefs, self-efficacy beliefs, outcome expectations and social interactions mediate the association between our cognitive behavioural group intervention and fear of falling and daily activity in community-living older people.

\section{Methods}

\section{Study design and sample}

The present study elaborated on a parallel-group randomised controlled trial that examined the effectiveness of a cognitive behavioural intervention on fear of falling and avoidance of activity due to fear of falling. The design and participant recruitment are described in detail elsewhere. ${ }^{13}$ In short, between November 2002 and July 2003 7,431 questionnaires to screen for eligibility were sent to random selections of the general older population living in two communities in The Netherlands. In total, 4,376 questionnaires were returned. ${ }^{2}$ Next, community-living people aged 70 or over reporting at least some fear of falling and some avoidance of activity due to fear of falling were included. People confined to bed, restricted by permanent use of wheelchair or waiting for nursing home admission were excluded. Of the 540 participants that enrolled in the trial, 280 were randomly allocated to a cognitive behavioural intervention and 260 to a control group that received no intervention. At baseline, directly after, and at 6 and 12 months after the intervention, data was independently collected using self-report questionnaires and structured telephone interviews. Outcome assessors were blinded for group allocation. ${ }^{13}$ Directly after the intervention, data was collected from 239 control and 232 intervention participants, at 6 months from 214 control and 208 intervention participants, and at 12 months from 209 control and 196 intervention participants. Reasons for withdrawal were similar in both groups. ${ }^{10}$ Written informed consent was obtained from all participants. The Medical Ethics Committee of the Maastricht University/Academic Hospital Maastricht approved the study protocol.

\section{Intervention}

The cognitive behavioural group intervention is aimed at reducing fear of falling and associated avoidance and comprises 8 weekly sessions of 2 hours and one booster session at 6 months after the intervention. After instilling realistic and adaptive views on falls risk and fear of falling, attention is paid to achieving behavioural change. ${ }^{9} 1718$ Strategies to achieve these aims included restructuring misconceptions to promote a 
view of fall risk and fear of falling as controllable, setting realistic goals for increasing activity, changing unsafe behaviour and the environment to reduce fall risk, and promoting physical exercise to increase strength and balance. ${ }^{9} 1718$ During the intervention different techniques and materials are applied including lectures, videos, group discussions, mutual problem solving, assertiveness training, physical exercises, and behavioural contracts and goal setting. Trained nurses qualified in geriatric care conducted the intervention in local community centres. More details about the intervention are described elsewhere. ${ }^{9} 131718$

\section{Measures}

\section{Outcome variables}

Fear of falling was assessed with a modified falls efficacy scale. ${ }^{19} 20$ This scale measured to what extent participants are concerned about falling during 14 activities of daily life such as cleaning the house, taking a bath or shower, using public transport, for example "How concerned are you that you might fall while cleaning the house?". At baseline the internal reliability of this scale was .91. Daily activity was assessed with the Frenchay Activity Index; a 15-item questionnaire that reflects the broader everyday activities of daily life, including domestic, leisure or work, and outdoor activities. ${ }^{21}$ Examples of these items comprise preparing main meals, light housework and local shopping. At baseline the internal reliability of this scale was .74.

Outcome variables were assessed at baseline, directly after, and at 6 and 12 months after the intervention.

\section{Mediator variables}

Two measures for control beliefs were applied: a 7-item generic mastery scale and a 4item domain-specific scale that assessed perceived control over falling. The mastery scale comprises items like "I have little control over the things that happen to me" and "There is little I can do to change many of the important problems I have". ${ }^{14}$ Control over falling was assessed by items as "I can overcome my fear of falling" and "There are things I can do to keep myself from falling". ${ }^{22}$ At baseline the internal reliability estimates of these scales were .69 and .76 , respectively.

With regard to self-efficacy beliefs, a general self-efficacy and a physical selfefficacy scale were applied. General self-efficacy beliefs were assessed by a 16-item scale..$^{23}$ Examples of these items are "If something looks too complicated, I will not even bother to try it" and "When I make plans, I am certain I can make them work". Physical self-efficacy beliefs were assessed by a 10-item scale that comprises items like "I have excellent reflexes" and "I have poor muscle tone". ${ }^{24}$ At baseline the internal reliability estimates of these self-efficacy scales were .81 and .66 , respectively.

Outcome expectations measures included perceived risk of falling and perceived consequences of falling questionnaires. The 3-item risk of falling questionnaire assesses the extent to which one expects to fall in the next year, for example "I think that it is likely that I will fall during the coming year". The consequences of falling questionnaire comprises two 6-item subscales assessing perceived loss of functional independence and perceived damage to identity after a fall. ${ }^{25}$ An example of the perceived consequences of falling functional subscale is "I will lose my independence" and of 
the psychosocial subscale is "I will be embarrassed". At baseline the internal reliability estimates of these three scales were $.74, .83$ and .54 , respectively.

To assess the extent of perceived social interactions from one's social network, we assessed the 12-item social support interactions questionnaire. ${ }^{26}$ This scale contains items like "Does it ever happen that people drop in for a pleasant visit?" and "Does it ever happen that people confide in you?". At baseline the internal reliability of this scale was .89.

All mediator variables were assessed at baseline, directly after, and at 6 and 12 months after the intervention.

\section{Covariates}

Several socio-demographic and health-related variables were assessed as covariates: age, gender, living situation (alone or not alone), educational level based on completed formal education and completed professional courses during lifetime, ${ }^{27} 28$ cognitive status, ${ }^{29}$ perceived general health ${ }^{30} 31$ and falls in the past 6 months. Cognitive status was assessed using an adapted version of the 11-item Telephone Interview for Cognitive Status (TICS). ${ }^{29}$ Age, gender, perceived general health and number of falls in the past 6 months are factors independently associated with fear of falling and avoidance of activity. ${ }^{2}$ Living situation, educational level and cognitive status are factors that, given intervention characteristics, potentially associate with the intervention effects. All covariates were assessed before randomisation.

\section{Statistical Analyses}

Descriptive statistics for control and intervention participants were computed for all variables. Then we explored the contribution of the longitudinally assessed mediator variables to the trajectories of fear of falling and daily activity in the cognitive behavioural intervention at direct, and 6 and 12 months follow-up. For this purpose we applied a method proposed by Baron and Kenny ${ }^{32}$ and examined in three sets of various mixedeffects linear regression models ${ }^{33}$ for each follow-up assessment: (1) whether intervention status was associated with each mediator variable (arrow 1 in Figure 1); (2) whether intervention status was associated with fear of falling and daily activity (arrow 2); and (3) whether intervention status was still associated with fear of falling and daily activity after adjustment for the mediator variable (arrows 1, 2 and 3). A variable is considered a mediator if the effect of the intervention on the outcome variable is reduced after controlling for the mediator. A mediator completely mediates the association between intervention and outcome variable if intervention effects disappear entirely after controlling for this mediator. ${ }^{32}$

In the first set of analyses we examined the effects of the intervention on the potential mediators adjusting for all covariates and possible differences in baseline values of the appropriate mediator. If $p<.05$, effect sizes were calculated by dividing the difference in mean group scores by the pooled standard deviation. Effect sizes of .20 are considered small, .50 medium and .80 large..$^{34}$ In the second set of analyses we examined the effectiveness of the intervention on fear of falling and daily activity while adjusting for all covariates and possible differences in values of the appropriate mediator and outcome variable. In the third set of analyses, data from three follow-up assessments of 
the appropriate mediator were added to the second set of regression models. However, if intervention effects were non-significant at the level of .10 or higher for either a mediator or an outcome variable, mediation effects are not presented due to a negligible impact. To study the contribution of the complete set of mediator variables, we repeated the second and third set of analyses and included all longitudinally assessed mediator variables in the models for fear of falling and daily activity. Lastly, we calculated the contribution of the mediator variables at direct, and 6 and 12 months follow-up by means of the percentage change in mean differences of the second and third set of analyses. The following equation was applied: ((mean difference ${ }^{1}-$ mean difference $\left.{ }^{2}\right)$ / mean difference 1) $\times 100 \%$. Here, mean difference ${ }^{1}$ refers to the mean difference obtained from the second set of analyses and mean difference ${ }^{2}$ refers to the mean difference obtained from the third set of analyses which included the potential mediators. Higher percentages of change indicate a larger contribution of the mediating variable in the association between the intervention and the outcome variable.

Overall, analyses were performed according to the intention-to-treat principle. Mediator and outcome variables were longitudinally assessed at direct follow-up, and at 6 and 12 months after the intervention and as such included in the mixed-effects regression models. Models accounting for within-person correlation over time using an unstructured covariance structure fitted the outcomes. Fixed effects for the interaction group $x$ time were examined to obtain insight into the effectiveness of the intervention at various follow-up assessments. Two-sided significance tests $(p<.05)$ were applied to all analyses. SPSS 14.0 was used in all analyses.

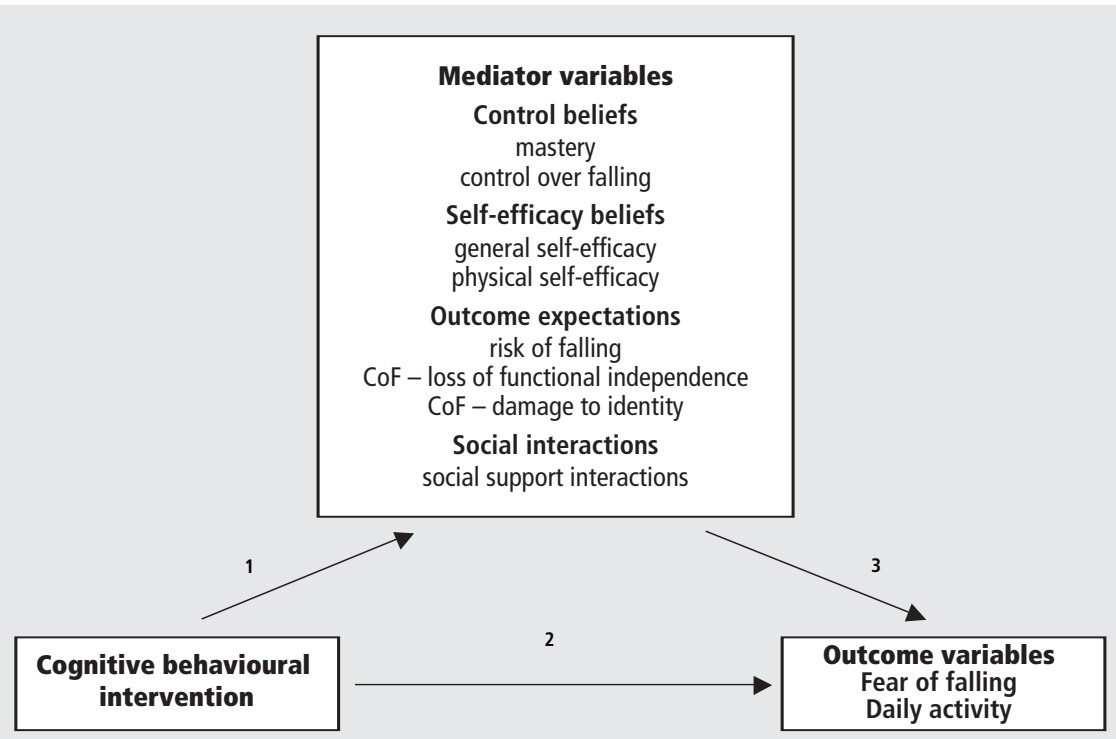

Figure 1 Model to explore the contribution of the mediator variables in the association between the intervention and outcome variables.

$\mathrm{COF}=$ perceived consequences of falling 


\section{Results}

\section{Baseline characteristics}

Baseline characteristics of the participants are presented in Table 1. The mean age of participants in both groups was approximately 78 years (range 70 to 92) and about $70 \%$ of the study sample consisted of women. The majority perceived their general health as fair or poor and nearly $45 \%$ of the participants had not suffered from a fall in the 6 months before baseline. The mean score for fear of falling was about 29 (range 14 to 56) and for daily activity about 39 (range 16 to 59). Overall, intervention and control group were comparable on most characteristics.

\section{Intervention effects on potential mediators}

Table 2 shows the effects of the cognitive behavioural group intervention on the potential mediators according to the intention-to-treat analyses. Compared to baseline scores, mean scores of the mediators improved at all follow-up assessments in the intervention group. Mixed-effects linear regression analyses showed favourable effects for the intervention group compared with the control group regarding every mediator at nearly all follow-up assessments. No statistically significant improvements were observed for control over falling and risk of falling directly after the intervention; for physical selfefficacy beliefs at 6 months; and for general self-efficacy beliefs and the loss of functional independence subscale at 12 months after the intervention. Overall, effect sizes were small to medium ranging from .04 for social interactions at 12 months follow-up to .41 for loss of functional independence at direct follow-up. Intervention outcomes on control over falling, outcome expectations and social interactions have previously been reported. ${ }^{10}$

\section{Intervention effects on outcomes}

In Table 3 intervention effects, in which we adjusted for all covariates and baseline values of the appropriate mediator and outcome, are presented in the first column of either fear of falling or daily activity. Generally, these mixed-effects linear regression analyses showed statistically significant intervention benefits regarding fear of falling and daily activity for the intervention group until 6 months after the intervention. At 12 months the intervention effects were nearly statistically significant for fear of falling $(p=.07)$. Intervention effects on fear of falling and daily activity without adjustment for possible differences in baseline values of the mediator variable have been reported elsewhere. ${ }^{10}$

\section{Mediating effects of psychosocial factors on fear of falling and activity}

The mediating effects of the longitudinally assessed psychosocial factors in the association between the intervention and fear of falling and daily activity are also presented in Table 3 (the second column of each outcome). Regarding fear of falling, estimates decreased after adjustment for the appropriate mediator. At direct follow-up intervention effects no longer reached the .05 level of statistical significance when general self-efficacy, loss of functional independence, damage to identity or the set of all mediators was added to the regression model. This also occurred at 12 months follow-up when physical self-efficacy beliefs, social interactions or the set of mediators was taken into account. With regard to the separate mediators, except for loss of 
Table 1 Baseline characteristics of participants $(N=540)$

\begin{tabular}{|c|c|c|}
\hline Characteristic* & $\begin{array}{l}\text { Control } \\
(n=260)\end{array}$ & $\begin{array}{l}\text { Intervention } \\
\quad(\mathrm{n}=280)\end{array}$ \\
\hline mean age in years at 1 January 2003 (SD) & $78.97(5.0)$ & 77.82 (4.6) \\
\hline number female (\%) & $190(73.1)$ & 198 (70.7) \\
\hline number living alone (\%) & $138(53.1)$ & $157(56.1)$ \\
\hline number low educational level $(\%)^{\dagger}$ & $153(59.1)$ & $185(66.1)$ \\
\hline mean cognitive status 0 to $\underline{41}$ (SD) & $32.22(3.9)$ & $31.69(3.6)$ \\
\hline number fair and poor perceived general health $(\%)^{\dagger}$ & $174(66.9)$ & 201 (71.8) \\
\hline number never fallen in the past 6 months $(\%)^{\dagger}$ & $117(45.0)$ & $123(43.9)$ \\
\hline
\end{tabular}

\section{Outcome variables}

mean fear of falling 14 to 56 (SD)

$29.97(10.2)$

$28.50(9.6)$

mean daily activity 15 to $\underline{60}$ (SD)

$38.16(7.2)$

$39.48(7.2)$

\section{Mediator variables}

Control beliefs

mean mastery 7 to $\underline{35}$ (SD)

$20.92(4.7)$

$21.45(4.6)$

mean control over falling 4 to $\underline{20}$ (SD)

13.09 (3.1)

13.48 (3.1)

Self-efficacy beliefs

$\begin{array}{llll}\text { mean general self-efficacy } 16 \text { to } \underline{80} \text { (SD) } & 54.35(11.0) & 54.14(11.3) \\ \text { mean physical self-efficacy } 10 \text { to } \underline{50} \text { (SD) } & 27.84(7.1) & 27.23(7.0)\end{array}$

Outcome expectations

$\begin{array}{lrrr}\text { mean risk of falling } \underline{3} \text { to } 12(S D) & 7.23(1.8) & 7.12 & (1.7) \\ \text { mean CoF - Ifi } \underline{6} \text { to } 24(S D)^{\ddagger} & 14.90(3.4) & 14.62 & (3.4) \\ \text { mean CoF - di } \underline{6} \text { to } 24(S D)^{\S} & 14.89(2.9) & 14.73 \quad(3.0)\end{array}$

Social interactions

mean social support interactions 12 to $\underline{48}$ (SD) $30.42(6.8) \quad 28.70 \quad(6.6)$

* The underlined score indicates the most favourable score. SD = standard deviation.

† Low, middle and high educational level; good, fair and poor perceived general health; never, once and more than once fallen.

₹ $\mathrm{CoF}-\mathrm{Ifi}=$ perceived consequences of falling - loss of functional independence.

$\S \mathrm{CoF}-\mathrm{di}=$ perceived consequences of falling - damage to identity.

functional independence and damage to identity, the percentage reductions in mean differences gradually increased over time. Overall, the percentage reduction ranged from 3\% for physical self-efficacy beliefs at direct follow-up to $54 \%$ for risk of falling at 12 months after the intervention. With regard to the set of mediators, estimates of the outcomes decreased substantially after adjustment for all mediators. The percentage change in estimates for fear of falling ranged from 44 to $76 \%$. 
Table 2 Effects of a cognitive behavioural intervention on potential mediators ( $N=540$ )

\begin{tabular}{|c|c|c|c|c|c|}
\hline & $\begin{array}{l}\text { Control } \\
\text { group }\end{array}$ & $\begin{array}{l}\text { Intervention } \\
\text { group }\end{array}$ & $\begin{array}{c}\text { Adjusted } \\
\text { mixed-effects }\end{array}$ & & $\begin{array}{c}\text { Effect } \\
\text { size }\end{array}$ \\
\hline Potential mediators ${ }^{\dagger}$ & Mean (SD) & Mean (SD) & $\begin{array}{c}\text { Mean } \\
\text { difference }(95 \% \mathrm{Cl})\end{array}$ & & $d$ \\
\hline \multicolumn{6}{|l|}{ Control beliefs } \\
\hline \multicolumn{6}{|l|}{ Mastery 7 to $\underline{35}$ (SD) } \\
\hline direct follow-up & $20.95(4.5)$ & $21.82(4.7)$ & $.84(.17-1.51)$ & * & .19 \\
\hline 6-month follow-up & $20.66(4.6)$ & $21.83(4.7)$ & $.95(.27-1.64)$ & ** & .25 \\
\hline 12-month follow-up & $20.60(4.9)$ & $21.91(5.0)$ & $1.14(.39-1.88)$ & ** & .26 \\
\hline \multicolumn{6}{|c|}{ Control over falling 4 to $\underline{20}$ (SD) } \\
\hline direct follow-up & $13.88(2.9)$ & $14.40(2.8)$ & $.46(-.02-.92)$ & + & - \\
\hline 6-month follow-up & $13.57(3.0)$ & $14.28(2.7)$ & $.73(.23-1.24)$ & ** & .25 \\
\hline 12-month follow-up & $13.46(3.1)$ & $14.42(2.9)$ & $.90(.38-1.43)$ & ** & .32 \\
\hline \multicolumn{6}{|c|}{ Self-efficacy beliefs } \\
\hline \multicolumn{6}{|c|}{ General self-efficacy 16 to $\underline{80}$ (SD) } \\
\hline direct follow-up & $54.52(11.0)$ & $56.42(11.0)$ & $2.25(.88-3.62)$ & ** & .17 \\
\hline 6-month follow-up & $53.68(11.7)$ & $56.70(11.2)$ & $2.81(1.18-4.44)$ & ** & .26 \\
\hline 12-month follow-up & $53.99(11.7)$ & $55.77(11.7)$ & $1.40(-.24-3.04)$ & $\#$ & - \\
\hline \multicolumn{6}{|c|}{ Physical self-efficacy 10 to $\underline{50}$ (SD) } \\
\hline direct follow-up & $28.00(7.3)$ & $29.37(7.5)$ & $1.54(.48-2.60)$ & ** & .19 \\
\hline 6-month follow-up & $27.68(7.2)$ & $27.98(7.6)$ & $.34(-.78-1.46)$ & & - \\
\hline 12-month follow-up & $27.32(7.3)$ & $28.33(7.7)$ & $1.42(.23-2.61)$ & * & .13 \\
\hline \multicolumn{6}{|c|}{ Outcome expectations } \\
\hline \multicolumn{6}{|c|}{ Risk of falling $\underline{3}$ to 12 (SD) } \\
\hline direct follow-up & $7.41(1.7)$ & $7.08(1.5)$ & $-.27(-.58-.04)$ & $\#$ & - \\
\hline 6-month follow-up & $7.52(1.7)$ & $6.99(1.4)$ & $-.44(-.73--.15)$ & ** & .35 \\
\hline 12-month follow-up & $7.50(1.5)$ & $7.00(1.5)$ & $-.44(-.75--.14)$ & ** & .33 \\
\hline \multicolumn{6}{|l|}{ CoF - Ifi $\underline{6}$ to $24(S D)^{\ddagger}$} \\
\hline direct follow-up & $15.15(3.4)$ & $13.82(3.1)$ & $-1.26(-1.80--.73)$ & ** & .41 \\
\hline 6-month follow-up & $15.32(3.5)$ & $14.14(3.2)$ & $-.88(-1.47--.30)$ & ** & .35 \\
\hline 12-month follow-up & $14.84(3.4)$ & $14.09(3.0)$ & $-.44(-1.02-.15)$ & & - \\
\hline \multicolumn{6}{|l|}{$\mathrm{CoF}-$ di $\underline{6}$ to $24(S D)^{\S}$} \\
\hline direct follow-up & $15.19(2.5)$ & $14.39(2.6)$ & $-.72(-1.13--.30)$ & $* *$ & .32 \\
\hline 6-month follow-up & $15.03(2.4)$ & $14.40(2.7)$ & $-.52(-.96--.09)$ & * & .25 \\
\hline 12-month follow-up & $15.07(2.5)$ & $14.32(2.7)$ & $-.63(-1.07--.19)$ & ** & .29 \\
\hline
\end{tabular}


Table 2 Effects of a cognitive behavioural intervention on potential mediators ( $N=540$ ) (continued)

\begin{tabular}{|c|c|c|c|c|}
\hline \multirow[b]{3}{*}{ Potential mediators $^{\dagger}$} & \multirow[b]{3}{*}{ Mean (SD) } & $\begin{array}{l}\text { Intervention } \\
\text { group }\end{array}$ & $\begin{array}{c}\text { Adjusted } \\
\text { mixed-effects }^{¥}\end{array}$ & $\begin{array}{c}\text { Effect } \\
\text { size }\end{array}$ \\
\hline & & & Mean & \\
\hline & & Mean (SD) & difference $(95 \% \mathrm{Cl})$ & $d$ \\
\hline \multicolumn{5}{|c|}{ Social interactions } \\
\hline \multicolumn{5}{|c|}{ Social support interactions 12 to $\underline{48}$ (SD) } \\
\hline direct follow-up & $29.46(7.2)$ & $29.99(6.1)$ & $1.47(.60-2.34) * *$ & .08 \\
\hline 6-month follow-up & $30.09(7.4)$ & $30.44(6.7)$ & $1.43(.50-2.37)$ ** & .05 \\
\hline 12-month follow-up & $29.70(7.5)$ & $29.96(6.6)$ & $1.35(.35-2.35)$ ** & .04 \\
\hline \multicolumn{5}{|c|}{$\# p<.10 ;+p<.075 ;{ }^{*} p<.05 ;{ }^{* *} p<.01$} \\
\hline $\begin{array}{l}¥ \text { Mixed-effects linear regres } \\
\text { status, perceived general hea } \\
\text { deviation. } \\
+ \text { The underlined score indice } \\
\neq \text { CoF }- \text { Ifi = perceived conse } \\
\S \text { CoF - di = perceived conse }\end{array}$ & $\begin{array}{l}\text { liusted for baseline } \\
\text { er of falls in the pa } \\
\text { avourable score. } \\
\text { ling - loss of funct } \\
\text { ling - damage to i }\end{array}$ & $\begin{array}{l}\text { lues and age, gend } \\
6 \text { months. } 95 \% \mathrm{Cl}= \\
\text { al independence. } \\
\text { tity. }\end{array}$ & $\begin{array}{l}\text { living situation, educational lev } \\
5 \% \text { confidence interval; SD = }\end{array}$ & $\begin{array}{l}\text { ognitive } \\
\text { ard }\end{array}$ \\
\hline
\end{tabular}

Regarding daily activity, at all assessments where intervention effects on daily activity and the appropriate mediator were observed $(p<.10)$, a decrease in the estimate was observed after taking the mediators into account. Intervention effects were no longer statistically significant $(p<.05)$ after adjustment for risk of falling, loss of functional independence or the set of all mediators at direct follow-up and after adjustment for general self-efficacy or loss of functional independence at 6 months follow-up. While the contribution of control beliefs, general self-efficacy beliefs or social interactions in the association between the intervention and daily activity appears to increase within the 6 months timeframe, the contribution of the outcome expectations or all mediators seems to decrease within this timeframe. Overall, the change in mean difference varied between 3 and $22 \%$ for the separate mediators and was about $45 \%$ for the set of all mediators.

\section{Discussion}

In the current study we explored the potential mediating effects of several longitudinally assessed psychosocial factors on trajectories of fear of falling and daily activity in a cognitive behavioural intervention. Previously this intervention has shown direct, favourable effects on fear of falling and daily activity until at least 6 months after the intervention. ${ }^{10}$ On the one hand these positive findings are encouraging as effective interventions in this field of research are scarce, on the other hand these positive findings 
Table 3 Effects of psychological mediators on fear of falling and daily activity $(N=540)$

\begin{tabular}{|c|c|c|c|c|c|c|}
\hline & & ar of falling & & & aily activity & \\
\hline Mediators & $\begin{array}{c}\text { Mean } \\
\text { difference }^{17}\end{array}$ & $\begin{array}{c}\text { Mean } \\
\text { difference }^{2 \dagger}\end{array}$ & $\%^{\ddagger}$ & $\begin{array}{c}\text { Mean } \\
\text { difference }^{1 ¥}\end{array}$ & $\begin{array}{c}\text { Mean } \\
\text { difference }^{2 \dagger}\end{array}$ & $\%^{\ddagger}$ \\
\hline Control beliefs & & & & & & \\
\hline Mastery & & & & & & \\
\hline direct follow-up & $-1.43 *$ & $-1.33 *$ & 7 & .94 * & .85 * & 10 \\
\hline 6-month follow-up & $-2.65 * *$ & $-2.46 * *$ & 7 & .93 * & .83 * & 11 \\
\hline 12-month follow-up & $-1.58+$ & -1.30 & 18 & .52 & - & - \\
\hline Control over falling & & & & & & \\
\hline direct follow-up & $-1.45 *$ & -1.34 * & 8 & .95 * & $.91 *$ & 4 \\
\hline 6-month follow-up & $-2.67 * *$ & $-2.31 * *$ & 13 & .95 * & .87 * & 8 \\
\hline 12-month follow-up & $-1.61+$ & -1.10 & 32 & .54 & - & - \\
\hline Self-efficacy beliefs & & & & & & \\
\hline General self-efficacy & & & & & & \\
\hline direct follow-up & $-1.45 *$ & $-1.28+$ & 12 & .95 * & .79 * & 17 \\
\hline 6-month follow-up & $-2.65 * *$ & $-2.32 * *$ & 12 & .94 * & $.73+$ & 22 \\
\hline 12-month follow-up & $-1.57+$ & -1.35 & 14 & .53 & - & - \\
\hline Physical self-efficacy & & & & & & \\
\hline direct follow-up & $-1.56 *$ & -1.52 * & 3 & $.99 * *$ & .89 * & 10 \\
\hline 6-month follow-up & $-2.79 * *$ & - & - & $.98 *$ & - & - \\
\hline 12-month follow-up & $-1.72 *$ & $-1.48+$ & 14 & .57 & - & - \\
\hline Outcome expectations & & & & & & \\
\hline Risk of falling & & & & & & \\
\hline direct follow-up & $-1.66 *$ & -1.54 * & 7 & .78 * & .65 & 17 \\
\hline 6-month follow-up & $-2.56 * *$ & -1.95 * & 24 & .66 & .56 & 15 \\
\hline 12-month follow-up & $-1.62+$ & -.75 & 54 & .30 & - & - \\
\hline $\mathrm{CoF}-\mathrm{Ifi}^{\S}$ & & & & & & \\
\hline direct follow-up & $-1.53 *$ & -.73 & 52 & .92 * & $.75+$ & 18 \\
\hline 6-month follow-up & $-2.81 * *$ & $-2.41 * *$ & 14 & .91 * & $.78+$ & 14 \\
\hline 12-month follow-up & $-1.47 \#$ & - & - & .51 & - & - \\
\hline $\mathrm{CoF}-\mathrm{di}^{\circ}$ & & & & & & \\
\hline direct follow-up & $-1.52 *$ & -1.01 & 34 & .95 * & .83 * & 13 \\
\hline 6-month follow-up & $-2.62 * *$ & $-2.36 * *$ & 10 & .93 * & .90 * & 3 \\
\hline 12-month follow-up & $-1.52+$ & -1.06 & 30 & .53 & - & - \\
\hline
\end{tabular}

warranted further study as the fairly modest effect sizes provide opportunities for intervention improvement. The findings of the current study showed moderate, favourable effects of the cognitive behavioural intervention on control beliefs, self- 
Table 3 Effects of psychological mediators on fear of falling and daily activity $(N=540)$ (continued)

\begin{tabular}{|c|c|c|c|c|c|c|}
\hline \multirow[b]{2}{*}{ Mediators } & \multicolumn{3}{|c|}{ Fear of falling } & \multicolumn{3}{|c|}{ Daily activity } \\
\hline & $\begin{array}{c}\text { Mean } \\
\text { difference }^{1 ¥}\end{array}$ & $\begin{array}{c}\text { Mean } \\
\text { difference }^{2 \dagger}\end{array}$ & $\%^{\ddagger}$ & $\begin{array}{c}\text { Mean } \\
\text { difference }^{1 ¥}\end{array}$ & $\begin{array}{c}\text { Mean } \\
\text { difference }^{2 \dagger}\end{array}$ & $\%^{\ddagger}$ \\
\hline \multicolumn{7}{|l|}{ Social interactions } \\
\hline \multicolumn{7}{|c|}{ Social support interactions } \\
\hline direct follow-up & $-1.53 *$ & $-1.53 *$ & 0 & 0.97 * & 0.88 * & 9 \\
\hline 6-month follow-up & $-2.74 * *$ & $-2.73 * *$ & 0 & 0.96 * & 0.86 * & 10 \\
\hline 12-month follow-up & $-1.67 *$ & $-1.55+$ & 7 & 0.56 & - & - \\
\hline \multicolumn{7}{|l|}{ All mediators } \\
\hline direct follow-up & $-1.86 *$ & -.56 & 70 & $.86 *$ & .41 & 52 \\
\hline 6-month follow-up & $-3.07 * *$ & $-1.71 *$ & 44 & $.78 \#$ & .46 & 41 \\
\hline 12-month follow-up & $-1.97 *$ & -.48 & 76 & .38 & - & - \\
\hline \multicolumn{7}{|c|}{$\# p<.10 ;+p<.075 ;{ }^{*} p<.05 ;{ }^{* *} p<.01$} \\
\hline \multicolumn{7}{|c|}{$\begin{array}{l}\text { - Not reported as the appropriate mediator or outcome variable was not affected by the intervention at the } p<.10 \text { level. } \\
¥ \text { Intervention effects analysed by mixed-effects linear regression models adjusted for baseline value of the outcome variable, age, } \\
\text { gender, living situation, educational level, cognitive status, perceived general health, number of falls in the past } 6 \text { months and the } \\
\text { baseline value of the appropriate mediator. }\end{array}$} \\
\hline \multicolumn{7}{|c|}{$\S \mathrm{CoF}$ - Ifi = perceived consequences of falling - loss of functional independence. } \\
\hline - $\mathrm{CoF}-\mathrm{di}=$ perceived cons & es of falling - dam & age to identity. & & & & \\
\hline
\end{tabular}

efficacy, outcome expectations and social interactions, and modest mediating effects of these psychosocial factors on fear of falling and daily activity in community-living older people.

A more detailed observation of the findings of the current study is warranted to facilitate better understanding of contributors to our cognitive behavioural intervention effects and further improvement of the intervention. First, effects of the psychosocial mediators were generally larger in fear of falling than in daily activity. This might be explained by the content and structure of our intervention in relation to these constructs. Concepts of cognitive restructuring received considerable attention during the sessions and participants' perceptions on falls and fall-related fear were strongly interwoven with session topics throughout the entire intervention. ${ }^{18}$ Yet, concepts of behavioural change, including increasing physical activity, were not addressed until about halfway the intervention and were restricted to limited parts of the sessions. Although change in behaviour was subsequently stimulated by contracting and goal-setting strategies, this might have been insufficient to achieve substantial changes in daily activity by the intervention. Second, change in outcome expectations explained the effects of the intervention on fear of falling to a large extent and on daily activity to a lesser extent. Next to these outcome expectations was control over falling at 12 months an apparent 
mediator for reduced fear of falling and general self-efficacy for daily activity. These findings suggest that, in our cognitive behavioural approach, fear of falling is mainly influenced by domain-specific fall-related perceptions and that this accounts to a lesser extent for daily activity. Increasing domain-specific expectations for enhancing activity, for instance by increased attention for physical activity, might be an important strategy to facilitate stronger and prolonged intervention effects regarding daily activity. Previous studies have shown the importance of thes eexpectations in increasing physical activity. ${ }^{35} 36$ Lastly, although the selected psychosocial factors explained a substantial part, that is 41 to $76 \%$, of the intervention effects, they never completely explained the association between the intervention and fear of falling or daily activity as reflected in the outcomes of the analyses in which we adjusted for all psychosocial factors simultaneously. This implies that our mediators lacked sufficient strength, that other factors (unaddressed in the current analyses) contributed to the relationship, or that both these explanations apply. With regard to lack of sufficient strength, in general, the separate mediating effects of control beliefs, self-efficacy beliefs, risk of falling and social interactions were small and appeared to increase over time, while those of perceived loss of functional independence and damage to identity were larger and reduced, or showed inconsistency over time. Achieving larger mediation effects more promptly after the intervention and subsequently maintaining them might be important for fully explaining the association between the intervention and outcomes. With regard to other mediating factors, to fully explain the association between the intervention and fear of falling or daily activity, other factors, such as activity-related outcome expectations and activity-related social support, might be important. These factors might not be limited to psychosocial aspects but might also comprise environmental and behavioural factors.

The aforementioned observations suggest that for reducing fear of falling and increasing daily activity, our current cognitive behavioural intervention might benefit from increased attention for the psychosocial mediators throughout the entire intervention. Consequently, increased attention for change in physical activity throughout the intervention, improved adherence to contracting and goal-setting strategies, and prolongation of the intervention might reinforce the current intervention. This intensified participation should increase participants' interaction of personal factors, environmental factors and behaviour, which according to the social cognitive theory, leads to improved self-efficacy beliefs and outcomes expectations. In addition, further study is warranted to search for additional mediators.

The current study has several strengths. First, as one of few, we explored the role of potential mediators in the association between an effective cognitive behavioural intervention and fear of falling and daily activity using adequate mediation methods. ${ }^{32}$ Despite the addressed importance of performing mediation analyses to accelerate future intervention improvement, reporting on underlying mechanisms is limited in general ${ }^{11} 37$ as well as specifically in studies on fear of falling. ${ }^{8}$ Second, the potential mediators were longitudinally assessed, that is until 12 months after the intervention, which provided insight into the intervention effects on these factors and the mediating effects of these factors on the outcomes over time. Next, intervention effects on the potential psychosocial mediators and their mediating effects were shown in our 
intention-to-treat analyses even though $42 \%$ of our intervention group withdrew before the start of the intervention or attended less than five sessions. ${ }^{1038}$ These effects support the strength of the intervention. Post-hoc mediation analyses in compliers with the intervention, that included participants attending at least five out of eight sessions, demonstrated similar trends in the mediating effects and slightly different percentages of mediator contributions (data not shown). Lastly, our potential mediators comprised several psychosocial factors based on the social cognitive theory, including outcome expectations, and comprised both generic and domain-specific constructs. Although common measures in fall-related research, such as the activity balance confidence scale $^{39}$ and the falls efficacy scale, ${ }^{40}$ comprise the aspect of self-efficacy beliefs of the social cognitive theory ${ }^{41}$, until now little was known about the importance of outcome expectations, another aspect of this theory. ${ }^{42} 43$

Some limitations of the current study are acknowledged and generated implications for future study. To begin with, although the underlying concepts of our mediator variables are different, there might be some overlap in the constructs. Overall, this overlap appears limited as the set of all mediator variables explained a substantial amount of the intervention effects. Secondly, in the current study we chose an explorative mediation method, as suggested by Baron and Kenny, ${ }^{32}$ that enabled comparison of the current mediation effects to intervention effects as previously reported. ${ }^{10}$ Future studies using more profound, but rather complex, analyzing techniques, such as structural equation modelling, might shed more light on the overlap in constructs and the causal pathway of the assessed mediators and outcomes over time. Moreover, although evidence is limited, falls self-efficacy has recently been shown to be an important mediator between an exercise intervention and fear of falling, ${ }^{44}$ and physical self-efficacy and social support as mediators between exercise interventions and physical activity. ${ }^{45} 46$ The latter two mediators explained merely to a very little amount our intervention effects on daily activity. Intervention improvement, such as intensifying adherence of intervention participants and addressing change in activity throughout the intervention, however, might reveal a more distinct role of these mediators in our intervention. We therefore recommend the assessment of these variables in future studies with similar interventions and outcomes, and recommend reporting on their contribution as mediators. In addition, in the current study we assessed fall-related outcome expectations, but omitted activity-related outcome expectations. Outcome expectations were the most apparent mediators and consequently we recommend assessing domain-specific outcome expectations in future studies on mediation processes in interventions, in addition to domain-specific self-efficacy beliefs. To conclude, although our intervention effects were small to modest, our set of selected mediators explained these effects to a substantial extent. To further improve our intervention effects addressing other potential mediators, for instance in the environmental domain, might be important. Future research in which a broader variety of potential mediators is addressed should shed a light on whether the theory underlying the intervention is comprehensive, as otherwise further development of the theory is warranted.

In conclusion, the current study showed that overall our cognitive behavioural intervention improved control beliefs, self-efficacy, outcome expectations and social 
interactions, and that these variables mediated the association between the intervention and fear of falling or daily activity in community-living older people. Generally, the separate contribution of these mediators to fear of falling and daily activity was modest. The set of all these mediators, however, explained the association between our cognitive behavioural intervention and fear of falling or daily activity to a substantial extent.

\section{Acknowledgements}

We gratefully acknowledge participants and facilitators for their involvement in our study. The Centre for Data and Information Management (MEMIC) and Vonca Schaffers are acknowledged for assistance in the data collection and dr. J.W.R. Twisk for statistical advice. This study was funded by ZonMw - The Netherlands Organisation for Health Research and Development (grant 014-91-052), the School for Public Health and Primary Care (Caphri), and the Faculty of Health, Medicine and Life Sciences of the Maastricht University. 


\section{References}

1. Tinetti ME, Speechley M, Ginter SF. Risk factors for falls among elderly persons living in the community. N Engl J Med 1988;319(26):1701-7.

2. Zijlstra GA, van Haastregt JC, van Eijk JT, et al. Prevalence and correlates of fear of falling and associated avoidance of activity in the general population of community-living older people. Age Ageing;36:304-9.

3. Friedman SM, Munoz B, West SK, et al. Falls and fear of falling: which comes first? A longitudinal prediction model suggests strategies for primary and secondary prevention. J Am Geriatr Soc 2002;50(8):1329-35.

4. Howland J, Peterson EW, Levin WC, et al. Fear of falling among the community-dwelling elderly. J Aging Health 1993;5(2):229-43.

5. Arfken CL, Lach HW, Birge SJ, et al. The prevalence and correlates of fear of falling in elderly persons living in the community. Am J Public Health 1994;84(4):565-70.

6. Mendes de Leon CF, Seeman TE, Baker DI, et al. Self-efficacy, physical decline, and change in functioning in community-living elders: a prospective study. J Gerontol B Psychol Sci Soc Sci 1996;51(4):S183-90.

7. Cumming RG, Salkeld G, Thomas M, et al. Prospective study of the impact of fear of falling on activities of daily living, SF-36 scores, and nursing home admission. J Gerontol A Biol Sci Med Sci 2000;55(5):M299-305.

8. Zijlstra GA, van Haastregt JC, van Rossum E, et al. Interventions to reduce fear of falling in community-living older people: a systematic review. J Am Geriatr Soc 2007;55:603-15.

9. Tennstedt S, Howland J, Lachman M, et al. A randomized, controlled trial of a group intervention to reduce fear of falling and associated activity restriction in older adults. J Gerontol B Psychol Sci Soc Sci 1998;53(6):P384-92.

10. Zijlstra GA, Van Haastregt JC, Ambergen T, et al. Effectiveness of a cognitive behavioural group intervention on fear of falling and associated avoidance of activity in communityliving older people: a randomised controlled trial. Submitted.

11. Kraemer HC, Wilson GT, Fairburn CG, et al. Mediators and moderators of treatment effects in randomized clinical trials. Arch Gen Psychiatry 2002;59(10):877-83.

12. Bandura A. Social foundation of thought and action: A social cognitive theory. Englewood Cliffs, NJ: Prentice Hall, 1986.

13. Zijlstra GA, van Haastregt JC, van Eijk JT, et al. Evaluating an intervention to reduce fear of falling and associated activity restriction in elderly persons: design of a randomised controlled trial [ISRCTN43792817]. BMC Public Health 2005;5(1):26.

14. Pearlin LI, Schooler C. The structure of coping. J Health Soc Behav 1978;19(1):2-21.

15. Bandura A. Self-efficacy. The exercise of control. New York: W.H. Freeman, 1997.

16. Bandura A, Adams NE, Beyer J. Cognitive processes mediating behavioral change. J Pers Soc Psychol 1977;35(3):125-39.

17. Tennstedt S, Peterson E, Howland J, et al. 'A Matter of Balance' - Managing Concerns about Falls. Boston: Roybal Center Consortium, Trustees of Boston University, 1998.

18. Zijlstra GA, Tennstedt SL, van Haastregt JC, et al. Reducing fear of falling and avoidance of activity in elderly persons: The development of a Dutch version of an American intervention. Patient Educ Couns 2006;62(2):220-7. 
19. Tinetti ME, Mendes de Leon CF, Doucette JT, et al. Fear of falling and fall-related efficacy in relationship to functioning among community-living elders. J Gerontol 1994;49(3):M140-7.

20. Hill KD, Schwarz JA, Kalogeropoulos AJ, et al. Fear of falling revisited. Arch Phys Med Rehabil 1996;77(10):1025-9.

21. Holbrook M, Skilbeck CE. An activities index for use with stroke patients. Age Ageing 1983;12(2):166-70.

22. Lawrence RH, Tennstedt SL, Kasten LE, et al. Intensity and correlates of fear of falling and hurting oneself in the next year: baseline findings from a Roybal Center fear of falling intervention. J Aging Health 1998;10(3):267-86.

23. Sherer M, Maddux JE. The Self-Efficacy Scale: Construction and Validation. Psychol Rep 1982;51:663-71.

24. Bosscher RJ, Laurijssen L, de Boer E. Measuring physical self-efficacy in old age. Percept Mot Skills 1993;77(2):470.

25. Yardley L, Smith H. A prospective study of the relationship between feared consequences of falling and avoidance of activity in community-living older people. Gerontologist 2002;42(1):17-23.

26. Kempen GI, Van Eijk LM. The psychometric properties of the SSL12-I, a short scale for measuring social support in the elderly. Soc Indic Res 1995;35(3):303-12.

27. CBS. Standaard Onderwijsindeling 1998. Editie 2001/'02. Voorburg/Heerlen: Centraal Bureau voor de Statistiek, 2001.

28. UNESCO. International Standard Classification of Education, ISCED. Paris: UNESCO, 1997.

29. Brandt J, Spencer M, Folstein M. The Telephone Interview for Cognitive Status. Neuropsychiatry Neuropsychol Behav Neurol 1988;1(2):111-7.

30. Stewart AL, Hays RD, Ware JE, Jr. The MOS short-form general health survey. Reliability and validity in a patient population. Med Care 1988;26(7):724-35.

31. Kempen GI, Brilman El, Heyink JW, et al. Het meten van de algemene gezondheidstoestand met de MOS Short-Form General Health Survey (SF-20): een handleiding. Groningen: Noordelijk Centrum voor Gezondheidsvraagstukken, Rijksuniversiteit Groningen, 1995.

32. Baron RM, Kenny DA. The moderator-mediator variable distinction in social psychological research: conceptual, strategic, and statistical considerations. J Pers Soc Psychol 1986;51(6):1173-82.

33. Verbeke G, Molenberghs G. Linear mixed models for longitudinal data. 1st ed. New York: Springer-Verlag, 2000.

34. Cohen J. A power primer. Psychol Bull 1992;112(1):155-9.

35. Resnick B, Orwig D, Magaziner J, et al. The effect of social support on exercise behavior in older adults. Clin Nurs Res 2002;11(1):52-70.

36. Resnick B. Health promotion practices of the old-old. J Am Acad Nurse Pract 1998;10(4):147-53.

37. Baranowski T, Lin LS, Wetter DW, et al. Theory as Mediating Variables: Why Aren't Community Interventions Working as Desired? Annual Epidemiology 1997; S7:S89-95.

38. van Haastregt JC, Zijlstra GA, van Rossum E, et al. Feasibility of a cognitive behavioural group intervention to reduce fear of falling and associated avoidance of activity in elderly people living in the community. BMC Health Serv Res 2007;7:156. 
39. Powell LE, Myers AM. The Activities-specific Balance Confidence (ABC) Scale. J Gerontol A Biol Sci Med Sci 1995;50a(1):M28-34.

40. Tinetti ME, Richman D, Powell L. Falls efficacy as a measure of fear of falling. J Gerontol 1990;45(6):P239-43.

41. Bandura A. Self-efficacy: toward a unifying theory of behavioral change. Psychol Rev 1977;84(2):191-215.

42. Lach HW. Self-efficacy and fear of falling: in search of complete theory. J Am Geriatr Soc 2006;54(2):381-2.

43. Yardley L, Kempen GI. Measuring expected outcomes of falls. J Am Geriatr Soc 2006;54(8):1300-1.

44. Li F, Fisher KJ, Harmer P, et al. Falls self-efficacy as a mediator of fear of falling in an exercise intervention for older adults. J Gerontol B Psychol Sci Soc Sci 2005;60(1):P34-40.

45. Lewis BA, Marcus BH, Pate RR, et al. Psychosocial mediators of physical activity behavior among adults and children. Am J Prev Med 2002;23(2 Suppl):26-35.

46. King $A C$, Taylor $C B$, Haskell $W L$, et al. Identifying strategies for increasing employee physical activity levels: findings from the Stanford/Lockheed Exercise Survey. Health Educ Q 1990;17(3):269-85. 

CHAPTER 9

General discussion 


\section{Introduction}

At the start of the studies presented in this thesis little was known about fear of falling and associated avoidance of activity in Dutch older people. An intervention to manage these concerns about falling for the Dutch setting was lacking. Internationally, only a few interventions aimed at reducing fear of falling and increasing physical activity were available. An American cognitive behavioural intervention, called 'A Matter of Balance', seemed promising for implementation in The Netherlands as this intervention: a) was specifically directed at community-living older people with fear of falling and associated avoidance of activity; b) included well-founded cognitive behavioural strategies; and c) showed favourable effects on falls efficacy and mobility in a randomised controlled trial. ${ }^{1}$ In short, this intervention of eight group sessions aims to increase self-efficacy beliefs regarding falls and the sense of control over falling. Several strategies are applied to achieve these aims, including restructuring misconceptions to promote a view of fall risk and fear of falling as controllable, setting realistic goals for increasing activity, changing unsafe behaviour and the environment to reduce fall risk, and promoting physical exercise to increase strength and balance.

The main objective of this thesis was to evaluate this cognitive behavioural intervention in community-living older people in the Dutch context. Yet, due to limited insight into fear of falling and avoidance of activity in this population and interventions suitable for reducing fear of falling in general, two objectives were added. Hence, the three objectives of this thesis are:

1. to study the prevalence and correlates of fear of falling and associated avoidance of activity in community-living older people in The Netherlands;

2. to obtain insight into the international literature on interventions that reduce fear of falling in community-living older people;

3. to evaluate a cognitive behavioural intervention aimed at reducing fear of

falling and associated avoidance of activity in community-living older people

in The Netherlands.

Several studies, as presented in Chapters 2 till 8, were performed with reference to these objectives. In the current chapter an overview and discussion of these studies is provided. First, the main findings of these studies are described. Next, strengths and limitations concerning the studies are discussed. Lastly, implications for practice and future research are provided, as well as some general conclusions.

\section{Main findings}

\section{Prevalence and correlates}

Results from the cross-sectional study among 4,031 community-living older people (Chapter 2) underscored the commonness of concerns about falling in this population: about 54\% reported fear of falling and nearly 38\% reported avoidance of activity due to fear of falling. Fear of falling and avoidance of activity due to this fear were independently associated with higher age, female gender, poorer perceived general health, and one or more falls in the past 6 months. Particularly people suffering from 
poor perceived general health or multiple falls in the past 6 months were more likely to experience fear of falling and associated avoidance of activity. These results are valuable for identifying people who are likely to suffer from concerns about falling and might benefit from an intervention on managing these concerns. The independent association between avoidance of activity and modifiable factors, like falls, suggested that these factors, in addition to fear of falling, might be important in strategies aimed at reducing avoidance of activity.

\section{Interventions to reduce fear of falling}

A systematic review was performed to obtain insight into interventions that reduce fear of falling in community-living older people (Chapter 3). Of 19 included randomised controlled trials, only three evaluated interventions that were explicitly aimed at reducing fear of falling. Reporting on methodological and process aspects was limited for items such as blinding of outcome assessor, concealment of treatment allocation, performance of the intervention according to protocol and feasibility of the intervention. Twelve trials were of higher methodological quality and in 11 of these trials, a statistically significant reduction in fear of falling was observed in the intervention group compared with the control group. The effective interventions included home-based fall-related multifactorial interventions, community-based tai chi interventions, home-based exercise interventions and a hip protector intervention.

\section{Evaluation of a cognitive behavioural intervention}

Before evaluation of the cognitive behavioural group intervention in The Netherlands, a translational study was performed to develop a Dutch version of the American cognitive behavioural intervention (Chapter 4). The process of adaptation was aimed at enhancing implementation of the intervention in the Dutch setting while preventing unnecessary deviations from the original intervention. Several predefined actions, e.g. expert consultation and conducting a pilot study, guided this process. Main adaptations that were made to the original intervention were scheduling more time for some activities, changing session frequency from twice a week to once a week, adding a booster session after 6 months and adding transparencies for visualisation of session contents.

Subsequently, this adapted intervention was evaluated in a parallel-group randomised controlled trial among Dutch community-living older people who reported at least some fear of falling and associated avoidance of activity (Chapter 5). Participants were randomly allocated to the control ( $n=260$ ) or intervention group ( $n=280$ ). Participants of the intervention group were invited to attend 8 weekly group sessions of 2 hours and a booster session at 6 months after the eighth session. Participants allocated to the control group received no intervention. To study short- and long-term effects of the intervention, participants received assessments at baseline, directly after the intervention, and at 6 and 12 months after the intervention. A variety of outcome measures were included in view of performing three studies.

First, a process evaluation was carried out to gain insight into the feasibility of the intervention in the Dutch setting (Chapter 6). Data were collected from 168 older people who participated in the intervention group and six facilitators. According to the facilitators, the majority of the sessions were performed according to protocol and no 
major deviations from the protocol were required. Of all participants allocated to the intervention group ( $n=280$ ), $42 \%$ attended less than five out of eight intervention sessions. Dropout before the start of the intervention and during the first sessions was considerable, 26 and $12 \%$ of the 280 participants, respectively. Yet, $84 \%$ of the participants who started the intervention completed it. The most common reasons for dropout were health problems. Participants reported their adherence to homework and physical exercises as good. Facilitators, however, rated the participants' adherence to these assignments less favourable. Directly after the intervention, participants and facilitators evaluated the overall intervention very positively and participants reported intervention benefits, like safe behaviour, and increased confidence and physical activity. The majority of the participants still reported substantial intervention benefits after 6 and 12 months. Recommendations from participants and facilitators indicated that simplifying the homework and adding extra sessions might improve the intervention.

Second, an evaluation was performed to obtain insight into the effectiveness of the cognitive behavioural intervention in community-living older people in The Netherlands with regard to several predefined primary and secondary outcomes (Chapter 7). Directly after the intervention data were collected from 239 control and 232 intervention participants, at 6 months from 214 control and 208 intervention participants, and at 12 months from 209 control and 196 intervention participants. Reasons for withdrawal were similar in both groups and the main reason reported for dropout during the trial was experiencing health problems. Data analysed according the intention-to-treat principle showed favourable intervention effects for the primary outcomes. These included fear of falling, avoidance of activity, fall-related self-efficacy and daily activity directly after the intervention; for these outcomes and perceived control over falling at 6 months; and for fear of falling and perceived control over falling at 12 months after the intervention. In addition, all secondary outcomes showed favourable effects at one or more of the follow-up assessments, except for perceived general health, self-rated life satisfaction and most outcomes assessed by fall calendar. Fall data from baseline until 12 months after the intervention showed statistically significant less recurrent fallers in the intervention group. Generally, effect sizes were small to medium (range .04 to .41). The per-protocol analyses, in which participants who attended at least five sessions were compared with the control group, showed additional favourable effects for the intervention group.

Third, the mediating effects of psychosocial factors on trajectories of fear of falling and daily activity in the Dutch cognitive behavioural intervention were studied (Chapter 8). Trajectories of control beliefs, self-efficacy beliefs, outcome expectations and social interactions were regarded as potential mediators. The outcomes showed that generally the cognitive behavioural intervention improved control beliefs, self-efficacy beliefs, outcomes expectations and social interactions, and that these variables mediated the association between the intervention and fear of falling or daily activity in communityliving older people. In general, the separate contribution of each mediator to the association between the cognitive behavioural intervention and fear of falling or daily activity was modest (range 3 to 54\%). The set of all mediators, however, explained these associations to a substantial extent (range 41 to $76 \%$ ).

To conclude, the evaluation of the cognitive behavioural intervention aimed at 
reducing fear of falling and associated avoidance of activity in community-living older people in The Netherlands showed encouraging results. A conscientious process of adaptation of the original intervention led to a Dutch cognitive behavioural intervention, which was considered feasible by participants and facilitators and which fitted well in regular care. Furthermore, the intervention yielded short- and long-term benefits for intervention participants regarding most outcomes, including fear of falling and avoidance of activity. However, as described in the discussion sections of the separate studies, minor refinement of the intervention is warranted to further improve intervention effectiveness and efficiency.

\section{Considerations}

As all studies are potentially susceptible to bias, reflection on strengths and limitations is warranted to facilitate interpretation and comparison of results. The strengths and limitations of the different studies of this thesis have been separately discussed in the previous chapters. For that reason, the current paragraph will mainly focus on some general considerations.

\section{Previous and current findings}

The randomised controlled trial presented in this thesis adds to the evidence on interventions aimed at reducing fear of falling. Consequently, findings of the current trial are reviewed in the light of previous findings, which are published in the systematic review on interventions to reduce fear of falling in community-living older people (Chapter 3). Table 1 presents a simplified overview of the trials that evaluated an intervention explicitly aimed at reducing fear of falling. As avoidance of activity due this fear of falling is an important aspect of this thesis, findings regarding avoidance of activity or increasing activity in daily life are now included as well.

Although the American and Dutch cognitive behavioural intervention are to a great extent comparable, evidence of the effectiveness of the Dutch intervention showed to be more robust. In the American intervention, most favourable effects regarding falls self-efficacy and mobility were only shown in compliers with the intervention and no effects were present at 6 months after the intervention. ${ }^{1}$ In contrast, at almost all follow-up assessments, the Dutch intervention showed favourable effects regarding fear of falling and daily activity in the intervention group as randomised. These differences in outcomes might be due to adaptations to the American intervention, such as adding a booster session and applying weekly sessions instead of twice weekly sessions. These adaptations might have led to increased exposure as participants had more time to apply obtained knowledge and skills in daily practice.

Comparing the two cognitive behavioural interventions to the multifactorial intervention of Clemson and colleagues, ${ }^{2}$ a substantial overlap is observed with regard to intervention content. Both types of interventions addressed fall-related aspects, such as physical exercise and hazards in the home environment, and applied techniques to manage these aspects, such as problem-solving and action planning. ${ }^{12}$ Consequently, similarities in findings are expected and are in fact shown for fear of falling at a follow- 
Table 1 Overview of trials aimed at reducing fear of falling

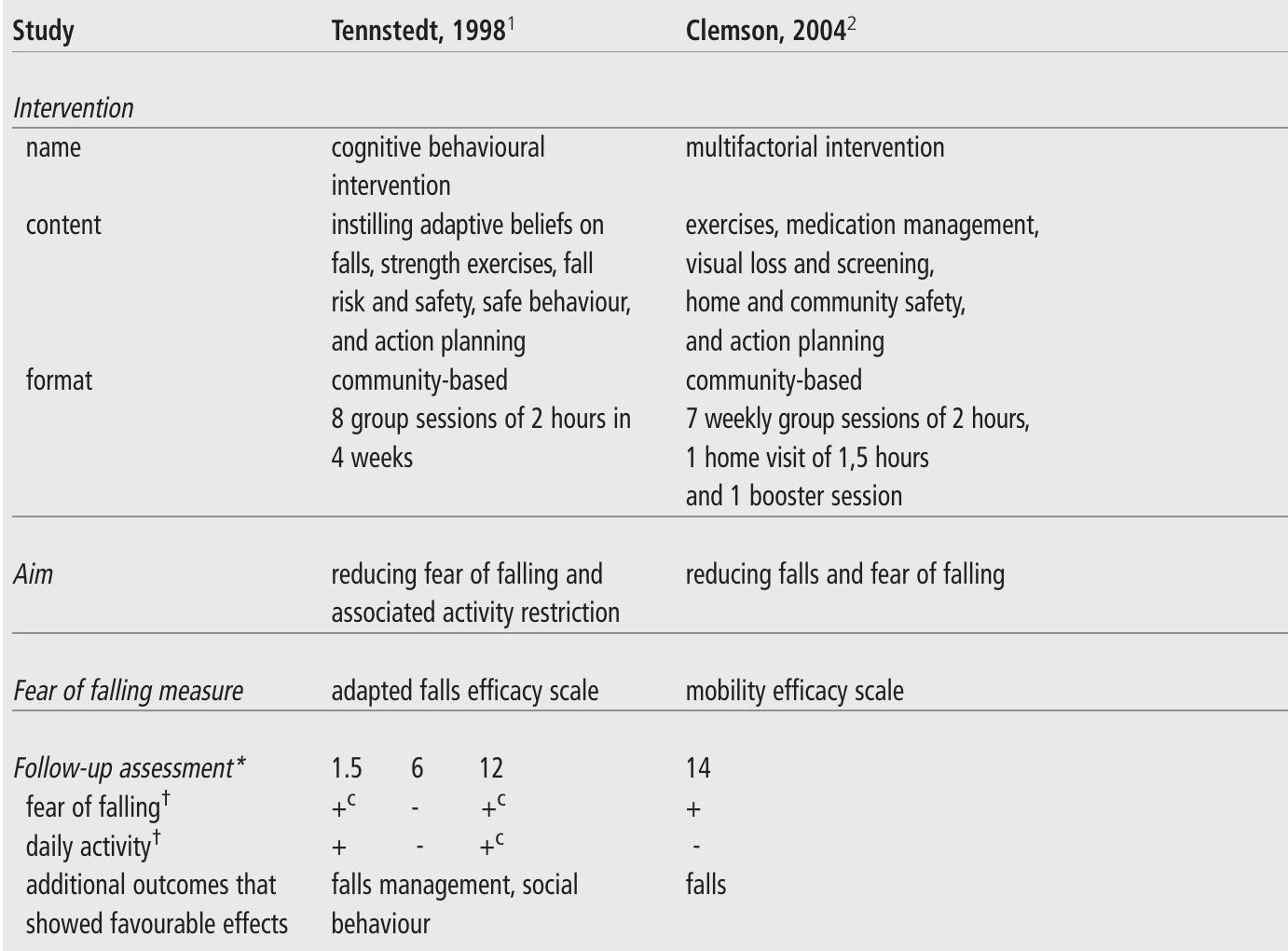

* in months after randomisation. $+^{c}=$ significant difference in favour of compliers with the intervention group.

${ }^{\dagger}+=$ significant difference in favour of intervention group. - = no significant difference between groups.

Significant difference if $p \leq .05$.

up after approximately 1 year. Yet, the lack of favourable effects regarding daily activity in the multifactorial intervention of Clemson and colleagues, in which compliance was considerable, is in contrast to the outcomes of the per-protocol analyses in the cognitive behavioural studies. This difference might be explained by somewhat more emphasis on psychological processes and behaviour change in the cognitive behavioural interventions and on physical and medical prevention strategies in the multifactorial intervention of Clemson and colleagues. Unfortunately, a comparison of findings prior to the 14-month follow-up is hampered by differences in study design, since Clemson and colleagues performed no intermediate assessments. ${ }^{2}$

Some additional aspects regarding the interventions aimed at reducing fear of falling, as presented in Table 1, are noted. First, the interventions have some features in common; all interventions are community-based, are conducted in group format, and, 
Zhang, $2006^{6}$ community-based

8-week programme of daily

group sessions of 1 hour
Zijlstra, submitted ${ }^{7}$

\section{Dutch cognitive behavioural} intervention

instilling adaptive beliefs on falls, strength exercises, fall risk and safety, safe behaviour, and action planning

community-based

8 weekly group sessions of 2

hours and 1 booster session increasing physical functioning

and reducing fear of falling

falls efficacy scale

2

$+$

not reported

balance, flexibility reducing fear of falling and associated avoidance of activity

fear of falling

$$
\begin{aligned}
& \begin{array}{lll}
2 & 8 & 14 \\
+ & + & + \\
+ & + & { }^{c}
\end{array} \\
& \text { recurrent falls, social support } \\
& \text { interactions, restriction of activities of } \\
& \text { daily life and other psychosocial } \\
& \text { outcomes }
\end{aligned}
$$

except for the intense tai chi intervention, consisted of approximately eight sessions. Second, all interventions showed to reduce fear of falling and in both interventions that facilitated a booster session, favourable effects regarding fear of falling were observed till 1 year after the intervention in the intervention group as randomised. Third, although the Dutch intervention showed favourable effects regarding daily activity till at least 6 months after the intervention, in both cognitive behavioural interventions only compliers benefited from increased daily activity until 1 year after the intervention. Lastly, all interventions yielded additional favourable outcomes. The American cognitive behavioural intervention mainly improved psychosocial outcomes and the multifactorial and tai chi intervention improved a limited number of outcomes in the physical domain. The Dutch cognitive behavioural intervention showed to improve a variety of outcomes in both the psychosocial and physical domain. 
The systematic review in Chapter 3 showed that even though most fall-related interventions were not explicitly aimed at reducing fear of falling, several types of interventions achieved favourable effects regarding this fear in community-living older people. In addition to favourable effects regarding fear of falling, the majority of these interventions also showed to improve their primary outcome (e.g. number of falls). Although not discussed in the systematic review, assessments of avoidance of activity or daily activities were uncommon and even if assessed, favourable effects regarding these activity measures, in addition to reducing fear of falling or falls, were very rarely reported. Yet, reporting activity levels is highly desirable as interventions may unintentionally lead people to restrict activities. This might result in a reduced number of falls as people are less exposed ${ }^{3}$ and to reduced fear of falling as people might avoid those activities in which fear of falling was experienced. In addition, if interventions reduce fear of falling to inappropriate levels, this might lead people to overestimate their capacities, ${ }^{4}$ which might initiate unsafe behaviour and subsequently may lead to increased number of falls. This indicates the importance of: a) promoting realistic and adaptive views on fall risk in older people, and b) obtaining favourable intervention effects on all three outcomes, i.e. fear of falling, activity and falls. These were all included in the Dutch trial.

\section{Measurement issues}

Many trials on mental and behavioural issues rely on self-reports of participants because objective measures are lacking or the disadvantages of applying objective measures, e.g. the costs or the extra burden for participants, are hampering their application. ${ }^{5}$ Outcomes in the trial described in this thesis relied on self-reports as well. A number of actions were undertaken to enhance reliability and validity of the self-reported data. First, primarily outcome measures that, according to the literature, showed good psychometric properties in older people were applied. Second, during the process evaluation, information was obtained from multiple sources, i.e. participants and facilitators. Third, a suitable administration method was applied to each outcome, for instance falls were registered by fall calendars to enable continuous registration and prevent recall bias. ${ }^{89}$ All methods were pilot-tested prior to the trial and were assessed in a structured format. Next, confidentiality of responses was warranted to increase openness in potential sensitive questions and to prevent socially desirable answers. Furthermore, all participants, including non-compliers with the intervention, were prompted to return measures and if measures were unreturned or contained missing data, participants were contacted to ensure completion of data. Lastly, assessors of the telephone interviews received training with respect to conducting the structured telephone interviews and were blinded for group allocation.

Although fear of falling is a generally accepted expression representing a concern about falling, a broad variety of concepts and associated measures of psychological aspects of falling have been applied over the past decade. ${ }^{10}$ These concepts have been used interchangeably in research. Yet recently, awareness has been raised on the potential differences between the various concepts, ${ }^{10} 11$ indicating the importance of providing information on the concept or different concepts assessed. In the trial described in this thesis, a variety of measures on psychological aspects of falling was 
assessed and intervention effects regarding these measures corresponded considerably. This may suggest that a similar concept is assessed. However, low correlations between fear of falling and other measures of psychological aspects of falling, and the extent to which these latter measures acted as mediators, indicate that these measures assess distinctive concepts. Additional analyses with respect to these different concepts and measures of psychological aspects of falling might provide more insight into the presence of a potential underlying concept. The variety in concepts and measures, however, warrants careful reporting of the exact psychological outcome of falling in future research.

Five of the 15 outcome measures as presented in the effect evaluation were assessed by single-item questions with limited answer options. The application of such questions can be considered a limitation as these items might conceal intervention effects due to insensitivity to change. This has previously been noted ${ }^{12}$ and appeared to be present in interventions to reduce fear of falling as well (Chapter 3 ). In the trial described in this thesis, however, the two single-item questions of the primary outcomes, i.e. fear of falling and associated avoidance of activity, did show favourable effects. These findings substantially correspond to findings as measured with the two multi-item scales: the fall-related self-efficacy scale, which assessed fear of falling while performing daily activities, and the daily activity scale. This overall correspondence strengthens the favourable findings. With regard to the secondary outcomes, the three single-item questions, i.e. perceived general health, self-rated life-satisfaction and feelings of loneliness, showed either no effects or small effects that diminished over time. Although this might be explained by insensitivity to change, this might also indicate that the cognitive behavioural intervention is insufficiently robust to actually affect these more distant outcomes. Future research in which multi-item scales are applied for the more distant outcomes might shed a light on this matter.

\section{Implications for interpretation and generalisation of effects}

Design and statistical analyses

Well-conducted randomised controlled trials are considered the most appropriate studies to assess the effectiveness of interventions. ${ }^{13}$ In such trials, random allocation serves as a method to obtain comparable groups, ${ }^{14}$ and blinding of participants, facilitators and outcome assessors as a method to prevent different kinds of bias. ${ }^{15}$ In the pragmatic trial presented in this thesis, random allocation was applied and baseline data showed that both groups were comparable after randomisation. Blinding was, however, limited to the outcome assessors as the surplus value of the intervention was studied. Standardisation of the intervention and assessments are regarded imperative methods to minimise bias if blinding is not optimal. ${ }^{14}$ Consequently, in the current trial such standardisation was performed. Due to the lack of an intervention aimed at reducing fear of falling and associated avoidance of activity in the Dutch context at the start of the trial, outcomes of the intervention group were compared with outcomes of the control group who received no intervention as part of the trial. Although a placebo control intervention may have facilitated blinding of participants, there are reasons not to conduct a distinct placebo intervention. ${ }^{16}$ One reason is to gain insight into the effects of the intervention in its entirety. In our cognitive behavioural 
intervention, social elements and attention, i.e. modelling and mutual problem solving, are considered key components of the intervention. Although controlling for these aspects by a placebo intervention might be appropriate in other trials, in the current trial this might have lead to biased outcomes, i.e. underestimated effects. Another reason not to conduct a placebo intervention in the control group is that such intervention is no realistic option in practice. ${ }^{14}$ Although it cannot be ruled out, it is unlikely that a placebo community-based intervention of eight sessions during which participants passively receive information on unrelated topics, would show results equivalent to the effects on the broad variety of outcomes of the cognitive behavioural intervention. This is underscored by several studies in which favourable effects were observed regarding fear of falling for the intervention group, ${ }^{17-19}$ compared with a placebo control group. In short, in the current trial blinding of participants and facilitators, which is considered a challenge in many intervention studies, ${ }^{15}$ was inappropriate and might have caused bias.

With regard to interpretation of the intervention effects, some statistical issues also deserve consideration. Longitudinal mixed-effects regression models were applied and statistical tests of significance were performed to reveal differences between both groups over time. Statistical significance is an indicator for the probability that differences between groups are observed by chance. If $p<.05$, the difference is regarded as a significant difference, i.e. an actual difference and not a finding by chance. Significant $p$ values, however, are no indicators for the clinical relevance or size of an effect. ${ }^{20}$ Reporting information on clinical relevance or effect sizes may facilitate interpretation of effects. Yet, determining whether our intervention effects are clinically relevant is complicated because no golden standards exist for levels of fear of falling and associated avoidance of activity. Therefore, effect sizes were presented. Cohen presented effect sizes of .20 as small, .50 as medium and .80 as large. ${ }^{21}$ According to this classification, the effect sizes of the cognitive behavioural intervention were generally small to medium, i.e. effect sizes ranged from .25 to .41 for the primary outcomes and from .04 to .41 for the secondary outcomes. A meta-analysis on the magnitudes of effect sizes in psychological, educational and behavioural interventions yielded a classification of effect sizes based on intervention outcomes; effect sizes of .00 to .32 were considered small, .33 to .55 medium and .56 to 1.20 large. ${ }^{22}$ According to this classification, effect sizes of the primary and fall-related outcomes of the cognitive behavioural intervention can be regarded as medium and effect sizes of all other outcomes as small. It is, however, acknowledged that even limited effect sizes can be important in daily life; ${ }^{23}$ this is underscored by the benefits reported by participants of our cognitive behavioural intervention (Chapter 6).

Table 2 shows an overview of effect sizes in different types of interventions that reduced fear of falling (Chapter 3). Reporting of effect sizes in these trials was scarce; therefore, for each trial the presented effect sizes on fear of falling were calculated by dividing the presented difference in mean group scores by the pooled standard deviation. The results showed fairly consistent effect sizes for each type of intervention except for the effect sizes shown in the multifactorial interventions. Comparing the different types of interventions brings to light the favourable effect sizes obtained by the tai chi interventions. Results of a trial in progress should provide insight into the 
Table 2 Simplified overview of effect sizes of fear of falling in different types of interventions

\begin{tabular}{|c|c|c|c|c|}
\hline Studies by intervention* & $\begin{array}{c}\text { Fear of falling } \\
\text { outcome }^{\dagger}\end{array}$ & $\begin{array}{c}\text { Fear of falling } \\
\text { concept }\end{array}$ & Follow-up ${ }^{\ddagger}$ & $\begin{array}{l}\text { Effect } \\
\text { size }^{\S}\end{array}$ \\
\hline \multicolumn{5}{|l|}{ Hip protector intervention } \\
\hline \multirow[t]{2}{*}{ Cameron, 2000, Australia ${ }^{38}$} & FES & falls self-efficacy & 4 & .43 \\
\hline & MFES & & midpoint & .40 \\
\hline \multicolumn{5}{|l|}{ Exercise intervention } \\
\hline Campbell, 1997, New Zealand ${ }^{17}$ & FES & falls self-efficacy & 12 & .16 \\
\hline Robertson, 2001, New Zealand 39 & MFES & falls self-efficacy & 12 & .26 \\
\hline \multicolumn{5}{|l|}{ Tai chi intervention } \\
\hline Li, 2005, USA ${ }^{1840}$ & $A B C$ & falls self-efficacy & 6 & .69 \\
\hline Zhang, 2006, Japan 6 & FES & falls self-efficacy & 2 & .61 \\
\hline \multicolumn{5}{|l|}{ Multifactorial intervention } \\
\hline Clemson, 2004, Australia² & MES & mobility self-efficacy & 14 & .17 \\
\hline \multirow[t]{2}{*}{ Tennstedt, 1998, USA ${ }^{1}$} & Adapted FES & falls self-efficacy & 1.5 & $.20^{\circ}$ \\
\hline & & & 12 & $.12^{\circ}$ \\
\hline Tinetti, 1994, USA 19 & Adapted FES & falls self-efficacy & 12 & .57 \\
\hline \multirow[t]{2}{*}{ Van Haastregt, 2000, Netherlands ${ }^{41}$} & Adapted FES & fear of falling & 12 & .20 \\
\hline & & & 18 & .23 \\
\hline \multirow[t]{3}{*}{ Zijlstra, Netherlands ${ }^{7}$} & Adapted FES & fear of falling & 2 & .27 \\
\hline & & & 8 & .41 \\
\hline & & & 14 & $.34^{\circ}$ \\
\hline
\end{tabular}

\footnotetext{
* Interventions that showed to reduce fear of falling using a continuous outcome (see Chapter 3).

${ }^{\dagger} \mathrm{FES}=$ Falls Efficacy Scale; 42 MFES = Modified Falls Efficacy Scale;43 ABC = Activities-specific Balance Confidence scale;44 MES = Mobility Efficacy Scale; ${ }^{2}$ Adapted FES = adapted version of FES, for instance activities in questions adapted to the population concerned.

₹ Follow-up measurement in months after randomisation. "Midpoint" refers to a measurement halfway through the intervention rather than after the intervention.

$\S$ Effect sizes (d) are calculated by dividing the difference in mean group scores by the pooled standard deviation. Effect sizes of interventions explicitly aimed at reducing fear of falling are presented in bold.

${ }^{\circ}$ Effect sizes as shown in compliers with the intervention.
}

potential effects and effect sizes of tai chi on fear of falling in Dutch community-living older people. ${ }^{24}$ The results in Table 2 and in Chapter 3 show that the interventions vary broadly with respect to several factors, e.g. effectiveness, intervention format and feasibility. Therefore, before implementing an intervention, careful consideration should be given to the most appropriate intervention for each setting. 
Table 3 Overview of dropout during the trial

\begin{tabular}{|c|c|c|}
\hline & $\begin{array}{l}\text { Control group } \\
\qquad(\mathrm{n}=260)\end{array}$ & $\begin{array}{l}\text { Intervention group } \\
\qquad(\mathrm{n}=280)\end{array}$ \\
\hline & Number (\%) & Number (\%) \\
\hline \multicolumn{3}{|l|}{ Assessments* } \\
\hline direct follow-up & $21(8.1)$ & 48 (17.1) \\
\hline 6-month follow-up & $46 \quad(17.7)$ & $72(25.7)$ \\
\hline 12-month follow-up & $51(19.6)$ & $84(30.0)$ \\
\hline \multicolumn{3}{|l|}{ Reasons for overall dropout } \\
\hline death & $6(2.3)$ & $6(2.1)$ \\
\hline health problems & $19(7.3)$ & $36(12.9)$ \\
\hline lost interest & $13(5.0)$ & $21(7.5)$ \\
\hline trial too burdensome & $6(2.3)$ & $21(7.5)$ \\
\hline life event significant other & $1(0.4)$ & $6(2.1)$ \\
\hline other reasons & $6(2.3)$ & $3(1.1)$ \\
\hline
\end{tabular}

\section{Participant selection}

Selection processes in a study population may limit the generalisation of findings and threaten internal validity. ${ }^{14}$ With regard to participant selection in the studies presented in this thesis, some issues merit consideration. The first issue entails the non-response in the cross-sectional study aimed to obtain insight into the prevalence and correlates of fear of falling and associated avoidance of activity in community-living older people. A random selection of the general population of people aged 70 years or older living independently in Maastricht and Heerlen was approached (Chapter 2). Little over 40\% of this population failed to return the questionnaire. Previous studies have shown that a variety of reasons might cause non-response in older people, for instance poor physical or mental functioning and lack of interest. ${ }^{25-27}$ In the current study insight into characteristics of non-responders was limited to age and gender, which showed to differ between responders and non-responders. With regard to the prevalence rates, these differences may imply that the prevalence rates of $54 \%$ for fear of falling and nearly $38 \%$ for associated avoidance of activity are underestimated. Nevertheless, these prevalence rates correspond to findings in various international studies, ${ }^{28-34}$ and, regarding fear of falling, also to findings in Dutch studies. ${ }^{35}{ }^{36}$ With regard to the correlates of fear of falling and associated avoidance of activity, the impact of the nonresponse is unlikely to limit generalisation of the findings as the impact of non-response on studied associations has previously shown to be quite small. ${ }^{37}$

The second issue concerns selection of participants during the randomised controlled trial (Chapter 5). The questionnaire applied in the cross-sectional study was used to efficiently select participants eligible for the trial. This implies that generalisations of trial outcomes are likely to be limited to the sample included in the trial. Yet, after 
randomisation at least two additional selection processes with regard to the participants warrant further consideration: 1) a selection in participants within the intervention group, and 2) a selection in participants due to dropout during the trial. With regard to the first matter, while initially interested in participating in the trial, $42 \%$ of the intervention group attended less than five sessions. A large proportion of these noncompliers attended less than two sessions. These problems with uptake and adherence have shown to be common in fall-related interventions. ${ }^{45}$ As previously recommended, non-compliers were approached for follow-up assessments and included in the intention-to-treat analyses. ${ }^{46}$ This type of analysis, however, may cause an underestimation of the magnitude of the intervention effects in compliant participants. ${ }^{47}$ The second matter, dropout of participants resulting in missing data, is regarded a more significant issue that can cause bias regarding intervention effects. While dropout during the trial described in this thesis was lower than estimated (Chapter 5), the percentage in dropout differed somewhat between both groups.

Table 3 presents an overview of the cumulative dropout during the trial and reasons for dropout in absolute numbers and percentages. The results show that the difference in dropout between both groups mainly occurred between baseline assessment and direct follow-up, and that health problems and trial demands were more frequently reported reasons for dropout in the intervention group. Although a placebo intervention was inappropriate for the research objectives of the current trial, in other trials a placebo intervention for the control group might lead to similar selection processes in participants, ${ }^{14}$ optimising comparison of groups. A systematic review in populationbased longitudinal studies in older people showed that dropout is frequently related to increased age and cognitive status. ${ }^{48}$ In the current trial, dropouts were more frail at baseline. In order to prevent bias due to selective dropout we included a number of relevant covariates in the mixed-effects regression models, e.g. age, cognitive status and perceived general health. In addition to several advantages, for instance allowing serial correlation and differences in time between follow-up assessments, the surplus value of applying mixed-effects regression models is the use of all available data. This implies that imputation techniques or deleting participants with incomplete data, which may be less efficient and may threaten validity, are avoided. Despite the relative novelty of this technique, the advantage and validity of applying mixed-effects regression techniques is noted. ${ }^{49}$ In addition, outcomes of mixed-effects models with missing data are considered comparable to outcomes of analyses with completed data. ${ }^{50}$ Overall, several efforts were undertaken to optimise compliance to the intervention, completion of data collection and analyses of available data, but like in other large trials, ${ }^{48}$ selection bias and missing data could not be completely prevented.

\section{Implications for practice and future research}

\section{Implications for practice}

The commonness and potential consequences of fear of falling and associated avoidance of activity in older people, in addition to the knowledge that these concerns about falling are not easily managed, warrants implementation of an effective 
prevention strategy in health care. In general, prevention strategies regarding the management of these concerns about falling are limited and in The Netherlands such a strategy was lacking. As a result the Dutch cognitive behavioural intervention described in this thesis, which facilitates community-living older people to properly manage their fear of falling and avoidance of activities due to this fear, is a valuable addition to current health care services in The Netherlands.

There are various aspects that facilitate or hamper implementation of interventions into regular care. ${ }^{51}$ First, with regard to effectiveness or relative advantage, the intervention reduced fear of falling and associated avoidance of activity, and showed favourable effects on a variety of other outcomes in community-living older people. Previous evidence regarding multifactorial interventions, in which cognitive behavioural techniques or action planning are applied in group sessions, ${ }^{12}$ strengthens the effectiveness of this type of intervention. The intervention can also be considered efficient due to the restricted number of sessions and time span. Compared to the aforementioned international exercise and tai chi interventions, which ranged from an 8-week tai chi intervention with daily sessions ${ }^{6}$ to exercising at least five times a week for 1 year, ${ }^{17} 39$ the cognitive behavioural intervention is considerably less demanding. Likely this is also reflected in costs associated with the different types of interventions. Next, with regard to utility and complexity, the intervention showed to be feasible, as judged by participants and facilitators, and functioned largely as expected, as shown by the contribution of mediators to the association between the intervention and fear of falling and daily activity. Lastly, with regard to compatibility and involvement, the intervention was developed in close collaboration with home care organisations in view of future implementation by these organisations. This implies that the intervention fits well into their methods of providing health care, and participants and facilitators are at hand. However, successful implementation of the intervention in the near future may be hampered by national changes in health care financing on the one hand, and health care professionals' unawareness of concerns about falling in older people and the availability of an intervention to manage these concerns on the other.

Organisations interested in implementing the cognitive behavioural intervention might benefit from experiences in the trial regarding enrolment of participants in the intervention. Although initially interested in participating in the trial, $26 \%$ of the participants who were invited to participate in the intervention withdrew before the start of the intervention. Health problems, considering the intervention inappropriate for their needs and other activities were the main reasons that kept people from taking up the intervention. This suggests that the applied method to enrol participants in the intervention, i.e. by written information and screening questions, was not fully successful in selecting the most appropriate population and motivating people to attend the intervention. In practice, a two-stage personalised strategy might improve uptake of the intervention by eligible older people. In the first stage potential participants may apply for the intervention either by means of self-referral or by means of referral by a health care professional, for instance a nurse providing home care, a general practitioner or a geriatrician. ${ }^{45}$ In the second stage a personal intake strategy is applied to check whether eligibility criteria are met, to provide information about the intervention, to motivate potential participants and to provide support with regard to 
finding solutions for factors that may hamper participation.

In addition to a personalised recruitment strategy, future providers of the intervention might also benefit from some implications for further improvement of the intervention that emerged from the trial. Both participants and facilitators underscored the importance of simplifying handouts and sheets to fully meet the capacities and skills of the participants. This might also increase adherence to homework and physical exercises, as facilitators indicated that adherence by the participants could be improved. Extra encouragement and assistance by the facilitator during sessions or via telephone after the sessions might also lead to an increase in adherence. These actions may facilitate increased confidence and improved skills in conducting the homework and physical exercises by the participants. In addition to these relatively limited changes to the intervention, some extensive changes might contribute to larger and long-term intervention effects. These changes may include increased duration of the intervention period and increased attention for control beliefs, self-efficacy beliefs, outcome expectancies and daily activity in the intervention protocol. Whether incorporation of extensive adaptations into the current intervention will lead to prolonged and larger effects without loss of feasibility, is uncertain. Therefore, an evaluation to establish whether such intervention is more efficient and effective than the current intervention is warranted. Given the benefits of the current cognitive behavioural intervention and the lack of an intervention to manage concerns about falls in community-living older people in The Netherlands, implementation of the current intervention after incorporating the limited refinements seems justified.

\section{Implications for future research}

Research on concerns about falling in older people has substantially increased in the past decade. Nevertheless, some issues can still be considered challenges for future studies. First, little is known about the initial development of fear of falling and associated avoidance of activity and their development over time, for instance with regard to severity. It seems plausible that factors associated with the process of aging, such as physical frailty, contribute to the initiation of these concerns about falling. Nevertheless, fear of falling and avoidance of activity may also precede factors like physical frailty. Assessing fear of falling and associated avoidance of activity in longitudinal studies on aging might shed light on these issues. Second, as aforementioned, over time different concepts have been applied to describe and assess psychological outcomes of falling. ${ }^{10}$ Yet, these concepts might not comprise one and the same psychological aspect of falling, ${ }^{101140}$ but may relate to a single underlying concept. ${ }^{52}$ Clarity with regard to these concepts is valuable, for instance to select the most appropriate outcome in a trial and to select appropriate mediators in an intervention to affect this outcome. Consequently, these issues warrant further study. Third, to facilitate comparison of outcomes in future trials, consensus should be obtained regarding a set of fall-related outcome measures, ${ }^{53}$ which are applicable in various countries and show good psychometric properties. Lastly, evidence with respect to the cost-effectiveness of interventions on fear of falling is still lacking, although such information will shortly be available for the Dutch cognitive behavioural intervention. Future trials should also assess cost-effectiveness to facilitate comparison between interventions. 
Several implications for future research emerged explicitly from the evaluation of the Dutch cognitive behavioural intervention. To begin with, future research should provide insight into the effectiveness of the intervention within different subgroups, for instance with regard to age, gender, educational level, cognitive status, perceived general health and previous falls. These analyses may shed a light on who is most likely to benefit from participation in the intervention. Recruitment strategies may then aim at including these participants. Accordingly, these analyses also indicate for whom the intervention requires adaptation to meet their needs or for whom a different intervention might be more appropriate. Tennstedt and colleagues performed such analyses and showed, among other findings, that people with greater concerns about falling were more likely to benefit from the American cognitive behavioural intervention. ${ }^{54}$ In second place, to obtain longterm intervention effects on fear of falling, daily activity and falls in older people with concerns about falling a reinforced cognitive behavioural intervention might be more appropriate. The current intervention might be reinforced by improved adherence and increased attention for psychosocial mediators and change in daily activity throughout the intervention. Extensive adaptations, however, warrant a thorough evaluation of the intervention before implementation into practice. In addition, implementation of the Dutch cognitive behavioural intervention into regular health care settings might benefit from implementation research. This research should provide insight into factors facilitating or hampering the process of implementation and might re-evaluate the feasibility of the intervention including the minor refinements. Next, considerable dropout and limited adherence of participants implies that further study of associations between factors assessed at baseline and these negative aspects is valuable. Knowledge about predictors of dropout and non-adherence might contribute to the development of appropriate and efficient recruitment strategies and methods to enhance adherence to the intervention. Lastly, for a substantial group of the target population health problems were the main reasons for dropout before and during the current intervention. Taking the targeted population into consideration, i.e. people aged 70 years or older and suffering from fear of falling and associated avoidance of activity, participating in a community-based intervention might not be appropriate for all, not even if transportation to the intervention is provided. For that reason, a tailor-made, home-based intervention to manage concerns about falls, which specifically meets the needs of frail older people who are homebound or consider a group intervention too burdensome, might be a valuable alternative to the community-based group intervention.

\section{General conclusions}

Fear of falling and avoidance of activity due to this fear are common concerns about falls in older community-living people in The Netherlands. Internationally, several interventions showed to reduce fear of falling. In The Netherlands, however, a prevention strategy to help older people manage their fear of falling and associated avoidance behaviour was lacking. A thorough process of adaptation of an American cognitive behavioural intervention, which showed promising results regarding the management of concerns about falls, led to a Dutch version of this intervention. 
Evaluation of this cognitive behavioural intervention aimed at reducing fear of falling and associated avoidance of activity showed encouraging results in community-living older people in The Netherlands. Participants and facilitators considered the intervention feasible and the intervention fitted well in regular care. A large number of participants, i.e. $84 \%$, who started the 8-week intervention, completed the intervention. Dropout before the intervention and lack of adherence to the intervention are, however, important concerns that require improvement. A recruitment strategy in which participants are referred to the intervention by health care professionals and receive a personal intake is considered helpful in tackling dropout. To obtain improvements in adherence additional measures, such as extra encouragement and assistance by facilitators or health care professionals, might be suitable.

The cognitive behavioural intervention showed positive and lasting effects on fear of falling and avoidance of activity. A variety of other outcomes, such as daily activity, anxiety, depression and recurrent falls, were also positively affected by the intervention. In addition, the intervention showed to affect control beliefs, self-efficacy, outcome expectations and social interactions. As hypothesised, these latter psychosocial factors mediated the association between the intervention and fear of falling and daily activity.

Overall, after minor refinements, implementation of the cognitive behavioural intervention in Dutch health care seems justified and valuable to help community-living older people manage their concerns about falls. 


\section{References}

1. Tennstedt S, Howland J, Lachman M, et al. A randomized, controlled trial of a group intervention to reduce fear of falling and associated activity restriction in older adults. $J$ Gerontol B Psychol Sci Soc Sci 1998;53(6):P384-92.

2. Clemson L, Cumming RG, Kendig $H$, et al. The effectiveness of a community-based program for reducing the incidence of falls in the elderly: a randomized trial. J Am Geriatr Soc 2004;52(9):1487-94.

3. Rubenstein LZ, Josephson KR, Trueblood PR, et al. Effects of a group exercise program on strength, mobility, and falls among fall-prone elderly men. J Gerontol A Biol Sci Med Sci 2000;55(6):M317-21.

4. Delbaere K, Crombez G, Van Den Noortgate N, et al. The risk of being fearful or fearless of falls in older people: An empirical validation. Disabil Rehabil 2006;28(12):751-6.

5. Long Foley K, Manuel J, Vitolins M. The utility of self-report in medical outcomes research. Evid Based Healthc Public Health 2005;9:263-4.

6. Zhang JG, Ishikawa-Takata K, Yamazaki H, et al. The effects of Tai Chi Chuan on physiological function and fear of falling in the less robust elderly: an intervention study for preventing falls. Arch Gerontol Geriatr 2006;42(2):107-16.

7. Zijlstra GA, Van Haastregt JC, Ambergen T, et al. Effectiveness of a cognitive behavioural group intervention on fear of falling and associated avoidance of activity in communityliving older people: a randomised controlled trial. Submitted.

8. Kosa J, Alpert JJ, Haggerty RJ. On the reliability of family health information: a comparative study of illness and related behaviour. Soc Sci Med 1967;1:165-81.

9. Coughlin SS. Recall bias in epidemiologic studies. J Clin Epidemiol 1990;43(1):87-91.

10. Jorstad EC, Hauer K, Becker $C$, et al. Measuring the psychological outcomes of falling: a systematic review. J Am Geriatr Soc 2005;53(3):501-10.

11. McKee KJ, Orbell $S$, Austin CA, et al. Fear of falling, falls efficacy, and health outcomes in older people following hip fracture. Disabil Rehabil 2002;24(6):327-33.

12. Yardley L, Beyer N, Hauer K, et al. Development and initial validation of the Falls Efficacy Scale-International (FES-I). Age Ageing 2005;34(6):614-9.

13. Cochrane Handbook for Systematic Reviews of Interventions 4.2.5. [updated May 2005]; Appendix 5b. Chichester: Wiley, 2005.

14. Bouter LM, Van Dongen MCJM. Epidemiologisch onderzoek: opzet en interpretatie. Utrecht: Bohn, Scheltema \& Holkema BV, 1988.

15. Schulz KF, Chalmers I, Hayes RJ, et al. Empirical evidence of bias. Dimensions of methodological quality associated with estimates of treatment effects in controlled trials. JAMA 1995;273(5):408-12.

16. Vickers AJ, de Craen AJM. Why use placebos in clinical trials? A narrative review of the methodological literature. J Clin Epidemiol 2000;53:157-61.

17. Campbell AJ, Robertson MC, Gardner MM, et al. Randomised controlled trial of a general practice programme of home based exercise to prevent falls in elderly women. BMJ 1997;315(7115):1065-9.

18. Li F, Harmer P, Fisher KJ, et al. Tai chi and fall reductions in older adults: a randomized controlled trial. J Gerontol A Biol Sci Med Sci 2005;60(2):187-94. 
19. Tinetti ME, Baker DI, McAvay G, et al. A multifactorial intervention to reduce the risk of falling among elderly people living in the community. N Engl J Med 1994;331(13):821-7.

20. Coe R. It's the effect size, stupid. What effect size is and why it is important. Retrieved 5 July 2007, from http://www.leeds.ac.uk/educol/documents/00002182.htm.

21. Cohen J. A power primer. Psychol Bull 1992;112(1):155-9.

22. Lipsey MW. Design sensitivity: statistical power for experimental research. Newbury Park, CA: Sage Publications, 1990.

23. Lipsey MW, Wilson DB. The efficacy of psychological, educational, and behavioral treatment: confirmation from meta-analysis. Am Psychol 1993;48(12):1181-209.

24. Zeeuwe PE, Verhagen AP, Bierma-Zeinstra SM, et al. The effect of Tai Chi Chuan in reducing falls among elderly people: design of a randomized clinical trial in The Netherlands [ISRCTN98840266]. BMC Geriatrics 2006;6:6.

25. Bowns I, Challis D, Tong MS. Case finding in elderly people: validation of a postal questionnaire. Br J Gen Pract 1991;41:100-4.

26. Leinbach RM. Alternatives to the face-to-face interview for collecting gerontological needs assessment data. Gerontologist 1982;22:78-82.

27. Hébert R, Bravo G, Korner-Bitensky N, et al. Refusal and information bias associated with postal questionnaires and face-to-face interviews in very elderly subjects. J Clin Epidemiol 1996;49(3):373-81.

28. Tinetti ME, Speechley M, Ginter SF. Risk factors for falls among elderly persons living in the community. N Engl J Med 1988;319(26):1701-7.

29. Howland J, Lachman ME, Peterson EW, et al. Covariates of fear of falling and associated activity curtailment. Gerontologist 1998;38(5):549-55.

30. Drozdick LW, Edelstein BA. Correlates of fear of falling in older adults who have experienced a fall. J Clin Geropsychol 2001;7(1):1-13.

31. Friedman SM, Munoz B, West SK, et al. Falls and fear of falling: which comes first? A longitudinal prediction model suggests strategies for primary and secondary prevention. J Am Geriatr Soc 2002;50(8):1329-35.

32. Murphy SL, Williams CS, Gill TM. Characteristics associated with fear of falling and activity restriction in community-living older persons. J Am Geriatr Soc 2002;50(3):516-20.

33. Yardley L, Smith H. A prospective study of the relationship between feared consequences of falling and avoidance of activity in community-living older people. Gerontologist 2002;42(1):17-23.

34. Fletcher PC, Hirdes JP. Restriction in activity associated with fear of falling among community-based seniors using home care services. Age Ageing 2004;33(3):273-9.

35. Stalenhoef PA, Diederiks JP, Knottnerus JA, et al. The construction of a patient recordbased risk model for recurrent falls among elderly people living in the community. Fam Pract 2000;17(6):490-6.

36. Pluijm SMF. Predictors and consequences of falls and fractures in the elderly. Vrije Universiteit Amsterdam, 2001.

37. Kempen Gl, van Sonderen E. Psychological attributes and changes in disability among low-functioning older persons: does attrition affect the outcomes? I Clin Epidemiol 2002;55(3):224-9.

38. Cameron ID, Stafford B, Cumming RG, et al. Hip protectors improve falls self-efficacy. Age Ageing 2000;29(1):57-62. 
39. Robertson MC, Devlin N, Gardner MM, et al. Effectiveness and economic evaluation of a nurse delivered home exercise programme to prevent falls. 1: Randomised controlled trial. BMJ 2001;322(7288):697-701.

40. Li F, Fisher KJ, Harmer P, et al. Falls self-efficacy as a mediator of fear of falling in an exercise intervention for older adults. J Gerontol B Psycho/ Sci Soc Sci 2005;60(1):P34-40.

41. van Haastregt JC, Diederiks JP, van Rossum E, et al. Effects of a programme of multifactorial home visits on falls and mobility impairments in elderly people at risk: randomised controlled trial. BMJ 2000;321(7267):994-8.

42. Tinetti ME, Richman D, Powell L. Falls efficacy as a measure of fear of falling. J Gerontol 1990;45(6):P239-43.

43. Hill KD, Schwarz JA, Kalogeropoulos AJ, et al. Fear of falling revisited. Arch Phys Med Rehabil 1996;77(10):1025-9.

44. Powell LE, Myers AM. The Activities-specific Balance Confidence (ABC) Scale. J Gerontol A Biol Sci Med Sci 1995;50a(1):M28-34.

45. Yardley L, Bishop FL, Beyer N, et al. Older people's views of falls-prevention interventions in six European countries. Gerontologist 2006;46(5):650-60.

46. Hollis $S$, Campbell F. What is meant by intention to treat analysis? Survey of published randomised controlled trials. BMJ 1999;319(7211):670-4.

47. Montori VM, Guyatt G. Intention-to-treat principle. CMAJ 2001;165(10):1339-41.

48. Chatfield MD, Brayne CE, Matthews FE. A systematic literature review of attrition between waves in longitudinal studies in the elderly shows a consistent pattern of dropout between differing studies. J Clin Epidemiol 2005;58:13-9.

49. Beunckens C, Molenberghs G, Kenward MG. Direct likelihood analysis versus simple forms of imputation for missing data in randomized clinical trials. Clin Trials 2005;2:379-86.

50. Twisk JW. Missing data in longitudinal studies. Applied longitudinal data analysis for epidemiology: a practical guide. Cambridge: Cambridge University press, 2003.

51. Grol R, Wensing M, editors. Implementatie. Effectieve verandering in de patiëntenzorg. Maarssen: Elsevier gezondheidszorg, 2001.

52. Yardley L, Donovan-Hall M, Francis K, et al. Attitudes and beliefs that predict older people's intention to undertake strength and balance training. J Gerontol B Psychol Sci Soc Sci 2007;62(2):119-25.

53. Lamb SE, Jorstad-Stein EC, Hauer K, et al. Development of a common outcome data set for fall injury prevention trials: the prevention of falls network europe consensus. J Am Geriatr Soc 2005;53(9):1618-22.

54. Tennstedt SL, Lawrence RH, Kasten L. An intervention to reduce fear of falling and enhance activity: who is most likely to benefit? Educ gerontol 2001;27:227-40. 

CHAPTER 10

Summary

Samenvatting

Co-authors and affiliations

Dankwoord / Acknowledgement

Publications

About the author 


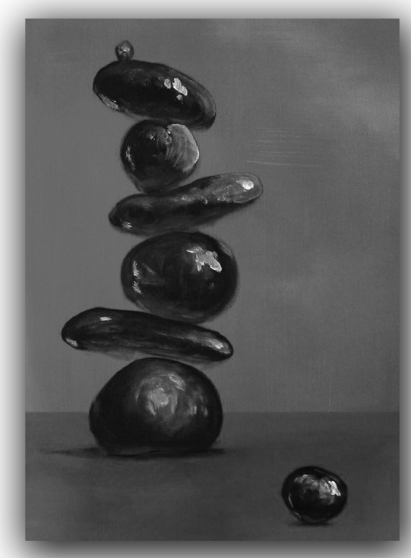




\section{Summary}

Aging societies will increasingly be challenged by health concerns prevalent in older populations. Fear of falling and associated avoidance of activity are regarded as health concerns. In general, about $50 \%$ of the community-living older people report fear of falling and about $40 \%$ report avoidance of activities due to this fear. These prevalence rates and the adverse consequences associated with fear of falling and avoidance behaviour, like decreased physical, psychological and social functioning, justify prevention strategies aimed at managing these concerns about falling. Internationally, little attention has been paid to the management of fear of falling and associated avoidance of activity in older people. In addition, at the start of the studies presented in this thesis little was known about fear of falling and avoidance of activity due to this fear of falling in Dutch older people and an intervention to manage these concerns about falling was lacking for the Dutch setting.

An American cognitive behavioural group intervention, called 'A Matter of Balance', seemed promising for implementation in The Netherlands as this intervention: a) was specifically directed at community-living older people with fear of falling and associated avoidance of activity; b) included well-founded cognitive behavioural strategies; and c) showed favourable effects on falls efficacy and activity in a randomised controlled trial in the United States. In short, this intervention of eight group sessions aims to increase self-efficacy beliefs regarding falls and the sense of control over falling. Several strategies are applied to achieve these aims, including restructuring misconceptions to promote a view of fall risk and fear of falling as controllable, setting realistic goals for increasing activity, changing unsafe behaviour and the environment to reduce fall risk, and promoting physical exercise to increase strength and balance. Success of an intervention in the United States is, however, no guarantee for its success in other countries. Therefore, an evaluation of this intervention adapted for the Dutch setting was desirable.

As described in Chapter 1, the main objective of this thesis was to evaluate a Dutch version of this cognitive behavioural intervention in community-living older people in The Netherlands. Yet, due to limited insight into fear of falling and associated avoidance of activity in this population and interventions suitable for reducing fear of falling in general, two objectives were added. Hence, the three objectives of this thesis are:

1. to study the prevalence and correlates of fear of falling and associated avoidance of activity in community-living older people in The Netherlands;

2. to obtain insight into the international literature on interventions that reduce fear of falling in community-living older people;

3. to evaluate a cognitive behavioural intervention aimed at reducing fear of falling and associated avoidance of activity in community-living older people in The Netherlands.

Chapter 2 describes the results of a cross-sectional study on the prevalence and correlates of fear of falling and associated avoidance of activity in community-living older people in The Netherlands (objective 1). A random selection of 7,431 people aged 70 years or over living independently in two communities, Maastricht or Heerlen, received a short questionnaire. Data from 4,031 responders to the questionnaire 
underscored the commonness of concerns about falling in community-living older people as about 54\% reported fear of falling and nearly $38 \%$ reported avoidance of activity due to fear of falling. Fear of falling and avoidance of activity due to this fear were independently associated with higher age, female gender, poorer perceived general health, and one or more falls in the past 6 months. Particularly people suffering from poor perceived general health or multiple falls in the past 6 months were more likely to experience fear of falling and associated avoidance of activity.

In Chapter 3 the findings of a systematic review performed to obtain insight into interventions that reduce fear of falling in community-living older people are presented (objective 2). An extensive, international search for relevant trials comprising database searches, expert consultations and searching reference lists, yielded 19 randomised controlled trials in which fear of falling was assessed. Only three interventions were explicitly aimed at reducing fear of falling. Reporting on methodological and process aspects was limited for items such as blinding of outcome assessor, concealment of treatment allocation, performance of the intervention according to protocol and feasibility of the intervention. Twelve trials were of higher methodological quality and in 11 of these trials, a statistically significant reduction in fear of falling was observed in the intervention group compared with the control group. The effective interventions included home-based fall-related multifactorial interventions, community-based tai chi interventions, home-based exercise interventions and a hip protector intervention.

Chapter 4 provides insight into the development process of a Dutch version of the American cognitive behavioural intervention aimed at reducing fear of falling and associated avoidance of activity in community-living older people. Several predefined actions, aimed at enhancing implementation of the intervention in the Dutch setting, while preventing unnecessary deviations from the original intervention, guided the process of adapting the American intervention. These actions comprised defining the target population, translating the original intervention manual literally, consulting Dutch experts and a developer of the original intervention, selecting qualified facilitators and conducting a pilot study of the adapted manual. The main adaptations included scheduling more time for some activities, changing session frequency from twice a week to once a week, adding a booster session after 6 months and adding more transparencies for visualisation of session contents.

Chapter 5 describes the design of the parallel-group randomised controlled trial, which was performed to evaluate the Dutch cognitive behavioural intervention (objective 3). In this trial community-living people 70 years of age or older received a short screening questionnaire and those reporting at least some fear of falling and associated avoidance of activity, and meeting other eligibility criteria were invited to participate. Directly after baseline assessment, participants were randomly allocated to the control $(n=260)$ or intervention $(n=280)$ group. Participants of the intervention group were invited to attend 8 weekly group sessions of 2 hours and a booster session at 6 months after the eighth session. All sessions were held in local community centres. Facilitators of the intervention were community nurses specialised in geriatric care and employed with two local homecare organisations. Participants allocated to the control group received no intervention. Data were collected in view of performing a process evaluation and effect evaluation of the intervention. The main outcomes of the process 
evaluation comprised performance of the intervention according to protocol, attendance and adherence of participants, and participants' and facilitators' opinion of the intervention. The main outcomes with respect to the effect evaluation were fear of falling, fall-related self-efficacy, perceived control over falling, associated avoidance of activity and daily activity. All data were gathered by means of self-administered questionnaires, telephone interviews, fall calendars and registration forms.

Chapter 6 presents the outcomes of the process evaluation, which was aimed at gaining insight into the feasibility of the intervention in the Dutch setting. Data were collected from 168 older people who participated in the intervention and six facilitators. According to the facilitators the majority of the sessions were performed according to protocol and no major deviations from the protocol were required. Of all participants allocated to the intervention group $(n=280), 42 \%$ attended less than five out of eight intervention sessions. Dropout before the start of the intervention and during the first sessions was considerable, 26 and $12 \%$ of the 280 participants respectively. Yet, $84 \%$ of the participants who started the intervention completed it. The most common reasons for dropout were health problems. Participants reported their adherence to homework and physical exercises as good. Facilitators, however, rated the participants' adherence to these assignments less favourable. Directly after the intervention, participants and facilitators evaluated the overall intervention very positively and participants reported intervention benefits, like safe behaviour, and increased confidence and physical activity. The majority of the participants still reported substantial intervention benefits after 6 and 12 months. Recommendations from participants and facilitators indicated that simplifying the homework and adding extra sessions might improve the intervention.

Chapter 7 reports on the effectiveness of the cognitive behavioural intervention in community-living older people in The Netherlands. Directly after the intervention data were collected from 239 control and 232 intervention participants, at 6 months from 214 control and 208 intervention participants, and at 12 months from 209 control and 196 intervention participants. The main reasons for dropout during the trial were health problems. Data analysed according the intention-to-treat principle showed favourable intervention effects regarding the primary outcomes. These included fear of falling, avoidance of activity, fall-related self-efficacy and daily activity directly after the intervention; for the latter outcomes and perceived control over falling at 6 months; and for fear of falling and perceived control over falling at 12 months after the intervention. Favourable effects at one or more of the follow-up assessments were also shown for a variety of other outcomes, such as restrictions in activities of daily life, anxiety, depression, perceived consequences of falling and recurrent falls. Generally, effect sizes were small to medium (range .04 to .41). The per-protocol analyses, in which participants who attended at least five sessions were compared with the control group, showed additional favourable effects for the intervention group.

In Chapter 8 the mediating effects of psychosocial factors on trajectories of fear of falling and daily activity in the Dutch cognitive behavioural intervention are presented. In this study control beliefs, self-efficacy beliefs, outcome expectations and social interactions were regarded as potential mediators. Longitudinal data of these psychosocial factors and the outcomes, i.e. fear of falling and daily activity, were 
applied in the mediation analyses. The outcomes showed that generally the cognitive behavioural intervention improved control beliefs, self-efficacy beliefs, outcomes expectations and social interactions, and that these variables mediated the association between the intervention and fear of falling or daily activity in community-living older people. In general, the separate contribution of each mediator to the association between the cognitive behavioural intervention and fear of falling or daily activity was modest (range 3 to 54\%). The set of all mediators, however, explained these associations to a substantial extent (range 41 to $76 \%$ ).

In Chapter 9 the main findings of this thesis are described, and strengths and limitations regarding the performed studies are discussed. In addition, implications for practice and future research were provided. On the whole, the cognitive behavioural intervention aimed at reducing fear of falling and associated avoidance of activity showed encouraging results in community-living older people in The Netherlands. Participants and facilitators considered the intervention feasible and the intervention fitted well in regular care. In addition, the cognitive behavioural intervention showed positive and lasting effects on fear of falling and avoidance of activity due to this fear. Overall, after minor refinements, implementation of the cognitive behavioural intervention in Dutch health care seems justified and valuable to help community-living older people manage their concerns about falls. 


\section{Samenvatting}

Samenlevingen met een vergrijzende populatie zullen in toenemende mate te maken krijgen met gezondheidsgerelateerde problemen bij ouderen. Angst om te vallen (valangst) en het vermijden van dagelijkse activiteiten als gevolg van deze angst is een voorbeeld van een dergelijk probleem. Resultaten van internationale studies laten zien dat valangst en gerelateerd vermijdingsgedrag veel voorkomen bij ouderen. Ongeveer de helft van de zelfstandig wonende ouderen rapporteert in meer of mindere mate bang te zijn om te vallen. Het vermijden van dagelijkse activiteiten als gevolg van angst om te vallen wordt door ongeveer $40 \%$ van de zelfstandig wonende ouderen gerapporteerd. Tevens laat internationaal onderzoek zien dat valangst en gerelateerd vermijdingsgedrag kan leiden tot een verminderd fysiek, mentaal en sociaal functioneren en tot vervroegde opname in een verpleeghuis. De hoge prevalentie en de potentiële impact van angst om te vallen en gerelateerd vermijdingsgedrag onderstrepen het belang van het nemen van effectieve maatregelen gericht op het omgaan met valangst en vermijdingsgedrag. In de internationale literatuur wordt echter weinig aandacht besteed aan de ontwikkeling en evaluatie van dergelijke interventies. Ook in Nederland was tot voor kort weinig bekend over angst om te vallen en gerelateerd vermijdingsgedrag bij ouderen en een behandelmethode was niet beschikbaar.

De cognitief gedragsmatige groepsinterventie 'A Matter of Balance', ontwikkeld en geëvalueerd in de Verenigde Staten, is één van de weinige interventies die zich specifiek richt op angst om te vallen en gerelateerd vermijdingsgedrag. Deze interventie liet positieve effecten zien op valgerelateerde eigen-effectiviteit, een maat voor valangst, en mobiliteit in een gerandomiseerde studie. Om deze reden lijkt implementatie van deze veelbelovende interventie in de Nederlandse situatie gerechtvaardigd. Samengevat bestaat de interventie uit acht groepsbijeenkomsten die gericht zijn op het verbeteren van de valgerelateerde eigen-effectiviteit en de ervaren controle over vallen. Er wordt gebruik gemaakt van verschillende methoden zoals het herstructureren van belemmerende gedachten over valrisico en angst om te vallen, het opstellen van realistische doelen gericht op het ondernemen van activiteiten, het veranderen van onveilig gedrag en het creëren van een veilige leefomgeving. Tevens worden lichamelijke oefeningen gedaan om de spierkracht en het balansvermogen te verbeteren. Het succes van de interventie in de Verenigde Staten biedt echter geen garantie dat deze interventie ook succesvol is in Nederland. Om deze reden werd een evaluatie van de Nederlandse versie van 'A Matter of Balance' wenselijk geacht voordat tot implementatie in het Nederlandse zorgaanbod kan worden overgegaan.

Het primaire doel van dit proefschrift is de evaluatie van een Nederlandse versie van de Amerikaanse cognitief gedragsmatige interventie bij zelfstandig wonende ouderen in Nederland (hoofdstuk 1). Hieraan zijn twee doelen toegevoegd vanwege de beperkte kennis over angst om te vallen en gerelateerd vermijdingsgedrag bij zelfstandig wonende ouderen in Nederland en vanwege het beperkte inzicht in interventies die angst om te vallen verminderen in de internationale literatuur. De drie doelstellingen van dit proefschrift zijn:

1. het onderzoeken van de prevalentie en voorspellers van angst om te vallen en gerelateerd vermijdingsgedrag bij zelfstandig wonende ouderen in Nederland; 
2. het verkrijgen van inzicht in interventies die angst om te vallen bij zelfstandig wonende ouderen verminderen zoals beschreven in de internationale literatuur;

3. het evalueren van een cognitief gedragsmatige interventie gericht op het verminderen van angst om te vallen en gerelateerd vermijdingsgedrag bij zelfstandig wonende ouderen in Nederland.

Hoofdstuk 2 beschrijft de resultaten van een cross-sectionele studie naar de prevalentie en voorspellers van angst om te vallen en gerelateerd vermijdingsgedrag in zelfstandig wonende ouderen in Nederland (doelstelling 1). Een willekeurige selectie van 7.431 zelfstandig wonende mensen van 70 jaar of ouder uit de gemeenten Maastricht en Heerlen ontving een korte vragenlijst. Uit de gegevens van de 4.301 teruggestuurde vragenlijsten bleek dat angst om te vallen veel voorkomt bij zelfstandig wonende ouderen. Ongeveer 54\% rapporteerde angst om te vallen en bijna 38\% rapporteerde het vermijden van dagelijkse activiteiten door angst om te vallen. Valangst en het vermijden van activiteiten vertoonde een onafhankelijke samenhang met een hogere leeftijd, het vrouwelijke geslacht, een slecht ervaren gezondheid en één of meer valincidenten in de afgelopen zes maanden. Vooral ouderen die vonden dat ze een slechte gezondheid hadden of ouderen die één of meer keer ten val kwamen in een voorafgaande periode van zes maanden hadden een grotere kans om angst om te vallen en gerelateerd vermijdingsgedrag te rapporteren.

In hoofdstuk 3 zijn de resultaten beschreven van een systematische 'review' die is uitgevoerd om meer inzicht te krijgen in interventies die angst om te vallen in zelfstandig wonende ouderen verminderen (doelstelling 2). Hiervoor werd een uitgebreid, internationaal literatuuronderzoek uitgevoerd naar relevante interventiestudies. Dit onderzoek bestond uit het doorzoeken van diverse literatuurbestanden, het consulteren van deskundigen op het gebied van valpreventie en het doorzoeken van referenties in literatuurlijsten. Uiteindelijk leverde dit 19 experimentele interventiestudies op. Slechts drie interventies richtten zich specifiek op het verminderen van angst om te vallen. In alle 19 studies werd relatief weinig gerapporteerd over zaken als het blinderen van de dataverzamelaars, het uitvoeren van een aselecte toewijzing van deelnemers aan groepen, het uitvoeren van de interventie volgens het protocol en de praktische uitvoerbaarheid van de interventie. Twaalf interventiestudies bleken van betere methodologische kwaliteit. In 11 van deze studies werd een vermindering van angst om te vallen waargenomen in de interventie groep ten opzichte van de controle groep. De effectieve interventies waren bewegingsinterventies, valgerelateerde multifactoriële interventies, tai chi interventies en een interventie met heupbeschermers.

Hoofdstuk 4 beschrijft de wijze waarop de Nederlandse versie van de Amerikaanse cognitief gedragsmatige interventie is ontwikkeld. Dit ontwikkelingsproces bestond uit de volgende, vastgestelde stappen: het definiëren van de doelgroep, het vertalen van het cursusprotocol, het consulteren van deskundigen, het selecteren van gekwalificeerde cursusleiders en het uittesten van het Nederlandse cursusprotocol bij een kleine groep ouderen $(n=11)$. De belangrijkste veranderingen ten opzichte van de Amerikaanse interventie zijn: het inplannen van extra tijd voor een aantal activiteiten, het aanpassen van de frequentie van de cursusbijeenkomsten van twee keer per week naar één keer per week, het toevoegen van een herhalingsbijeenkomst 6 maanden na afloop van de interventie en het toevoegen van overhead sheets voor visuele weergave van de cursusinhoud. 
Hoofdstuk 5 beschrijft de onderzoeksopzet van de gerandomiseerde experimentele studie naar de effecten van de Nederlandse interventie (doelstelling 3). Zelfstandig wonende mensen van 70 jaar of ouder, die valangst en gerelateerd vermijdingsgedrag rapporteerden in een korte vragenlijst, werden uitgenodigd deel te nemen aan het onderzoek. Direct na de voormeting werden de deelnemers aselect toegewezen aan de controle groep ( $n=260$ ) of interventie groep $(n=280)$. De mensen in de interventie groep werden uitgenodigd deel te nemen aan de cursus 'Beter in Balans'. Deze cursus bestond uit 8 wekelijkse groepsbijeenkomsten van 2 uur en één herhalingsbijeenkomst 6 maanden na afloop van de achtste bijeenkomst. Alle bijeenkomsten werden in een plaatselijk wijkgebouw of zorgcentrum georganiseerd. De cursus werd geleid door wijkverpleegkundigen met een specialisatie in de ouderenzorg en werkzaam bij een thuiszorgorganisatie. De deelnemers die waren toegewezen aan de controle groep ontvingen geen interventie. Tijdens de studie is zowel een procesevaluatie als een effectevaluatie uitgevoerd. Metingen vonden voor beide groepen plaats op vier momenten: voor de start van de interventie, direct na afloop van de interventie en vervolgens 6 en 12 maanden na afloop van de interventie. Alle gegevens zijn verzameld met vragenlijsten, telefonische interviews, valkalenders en registratieformulieren.

Hoofdstuk 6 beschrijft de resultaten van de procesevaluatie waarin werd nagegaan in hoeverre de interventie praktisch uitvoerbaar is in de Nederlandse situatie. Hiervoor zijn bij 168 deelnemers uit de interventie groep en bij zes cursusleiders gegevens verzameld. De belangrijkste uitkomstmaten van de procesevaluatie waren de mate waarin de interventie volgens protocol werd uitgevoerd, de mate van aanwezigheid van de deelnemers bij de bijeenkomsten, de mate waarin de deelnemers de huiswerkopdrachten uitvoerden en de mate van tevredenheid van de deelnemers en wijkverpleegkundigen over de interventie. Volgens de cursusleiders is de meerderheid van alle bijeenkomsten uitgevoerd volgens het cursusprotocol en is er niet substantieel van het protocol afgeweken. Van alle deelnemers uit de interventie groep $(n=280)$ is $42 \%$ minder dan vijf van de acht bijeenkomsten aanwezig geweest. Voor de start van de interventie en tijdens de eerste bijeenkomsten viel een aanzienlijk deel van de deelnemers uit, respectievelijk 26 en 12\%. Het hebben van een gezondheidsprobleem was een veel genoemde reden om niet aan de cursus te beginnen of met de cursus te stoppen. Van de deelnemers die wel startten, makkte $84 \%$ de interventie af. De deelnemers vonden dat ze, in het algemeen, de huiswerkopdrachten en lichamelijke oefeningen in de thuissituatie goed uitvoerden. De cursusleiders waren minder positief over de uitvoering van deze onderdelen van de cursus door de deelnemers. Direct na afloop werd de cursus positief beoordeeld door zowel de deelnemers als de cursusleiders. Deelnemers gaven aan baat te hebben gehad bij de cursus. Men gaf bijvoorbeeld aan dat men zich veiliger was gaan gedragen, meer zelfvertrouwen had gekregen en lichamelijk actiever was geworden. Na 6 en 12 maanden gaf de meerderheid van de deelnemers aan nog steeds aanzienlijk baat te ondervinden van deelname aan de cursus. Als aanbevelingen voor het verbeteren van de cursus noemden de deelnemers en cursusleiders het vereenvoudigen van de huiswerkopdrachten en het toevoegen van extra bijeenkomsten.

Hoofdstuk 7 beschrijft de resultaten van de effectevaluatie van de cognitief gedragsmatige interventie bij zelfstandig wonende ouderen in Nederland. De belangrijkste uitkomstmaten van de effectevaluatie waren angst om te vallen, ervaren controle over 
vallen, het vermijden van activiteiten als gevolg van angst om te vallen en dagelijkse activiteiten. De gegevens werden verzameld op drie momenten: direct na afloop van de interventie, na 6 maanden en na 12 maanden. Aan deze drie metingen deden respectievelijk 239, 214 en 209 deelnemers uit de controle groep en 232, 208 en 196 deelnemers uit de interventie groep mee. Vooral het hebben van gezondheidsproblemen was een belangrijke reden om te stoppen met het onderzoek. De verzamelde gegevens zijn volgens het 'intention-to-treat' principe geanalyseerd; dit betekent dat alle deelnemers van wie gegevens beschikbaar waren zijn meegenomen in de analyses, ongeacht hun deelname aan de cursus. De mensen in de interventie groep bleken beter te scoren op de primaire uitkomsten dan de mensen in de controle groep. Direct na afloop rapporteerde men minder angst om te vallen, minder vermijdingsgedrag als gevolg van deze angst en voerde men meer activiteiten uit. Zes maanden later was dit nog steeds het geval en ervoer men daarnaast ook meer controle over vallen. Na 12 maanden was men nog steeds minder bang om te vallen en ervoer men meer controle over vallen. Naast deze effecten waren er ook positieve effecten zichtbaar op één of meerdere meetmomenten voor verschillende andere uitkomstmaten, zoals ervaren beperkingen bij activiteiten van het dagelijkse leven, gevoelens van angst, symptomen van depressie, ervaren gevolgen van vallen en herhaalde valincidenten. De effecten waren klein tot middelmatig. In aanvulling op de 'intention-to-treat' analyses zijn 'per-protocol' analyses uitgevoerd, waarin deelnemers die vijf of meer cursusbijeenkomsten hadden bijgewoond, werden vergeleken met deelnemers uit de controle groep. Bij deze analyses werden meer en sterkere effecten van de cursus aangetoond.

In hoofdstuk 8 zijn de mediërende effecten van psychosociale factoren op het beloop van angst om te vallen en dagelijkse activiteiten als gevolg van de interventie beschreven. In dit onderzoek zijn ervaren controle, ervaren eigen-effectiviteit, valgerelateerde uitkomstverwachtingen en sociale interacties beschouwd als mogelijke mediatoren. Hierbij is gebruik gemaakt van de longitudinaal verzamelde gegevens uit de experimentele studie. Over het algemeen zorgde de interventie voor positieve effecten ten aanzien van de ervaren controle, de ervaren eigen-effectiviteit, de valgerelateerde uitkomstverwachtingen en de sociale interacties. Deze factoren bleken ook inderdaad mediatoren te zijn in de relatie tussen de cognitief gedragsmatige interventie en angst om te vallen of dagelijkse activiteiten bij zelfstandig wonende ouderen. De afzonderlijke bijdrage van elke mediërende factor op valangst of dagelijkse activiteiten varieerde tussen 3 en 54\%. Wanneer alle mediatoren werden meegenomen in de analyses bleken zij aanzienlijk bij te dragen aan de relatie tussen de interventie en angst om te vallen of dagelijkse activiteiten. De afname in valangst en toename in dagelijkse activiteiten bij deelnemers aan de interventie werd voor 41 tot $76 \%$ verklaard door de psychosociale factoren.

In hoofdstuk 9 zijn de belangrijkste bevindingen van dit proefschrift beschreven en komen de sterke en zwakke punten van de uitgevoerde studies aan bod. Op basis van de resultaten van de studies worden aanbevelingen gedaan voor de praktijk en toekomstig onderzoek. Samengevat liet de cognitief gedragsmatige positieve, langdurige resultaten zien ten aanzien van valangst, gerelateerd vermijdingsgedrag, dagelijkse activiteiten en een aantal secundaire uitkomstmaten, waaronder herhaalde valincidenten, bij zelfstandig wonende ouderen in Nederland. Deelnemers en cursusleiders vonden de interventie goed uitvoerbaar en de interventie bleek goed inpasbaar in het bestaande 
zorgstelsel. Afsluitend kan gezegd worden dat, na enkele kleine aanpassingen van het protocol, implementatie van de cognitief gedragsmatige groepsinterventie in de Nederlandse gezondheidszorg gerechtvaardigd en waardevol lijkt. 


\section{Co-authors and affiliations}

The co-authors of the studies described in this thesis and their current and primary, research affiliations are:

$\mathrm{T}$ (Ton) Ambergen, PhD

Maastricht University, Faculty of Health, Medicine and Life Sciences, Department of Methodology and Statistics, School for Public Health and Primary Care (Caphri), Maastricht, The Netherlands

JThM (Jacques) van Eijk, PhD

Maastricht University, Faculty of Health, Medicine and Life Sciences, Department of Social Medicine, School for Public Health and Primary Care (Caphri), Maastricht, The Netherlands

JCM (Jolanda) van Haastregt, PhD

Maastricht University, Faculty of Health, Medicine and Life Sciences, School for Public Health and Primary Care (Caphri), Maastricht, The Netherlands

GIJM (Ruud) Kempen, PhD

Maastricht University, Faculty of Health, Medicine and Life Sciences, School for Public Health and Primary Care (Caphri), Maastricht, The Netherlands

E (Erik) van Rossum, PhD

Professional University Zuyd, Faculty of Health and Technology, Heerlen and Maastricht University, School for Public Health and Primary Care (Caphri), Maastricht, The Netherlands

PA (Paul) Stalenhoef, MD, PhD

Maastricht University, Faculty of Health, Medicine and Life Sciences, Department of General Practice, School for Public Health and Primary Care (Caphri), Maastricht, The Netherlands

SL (Sharon) Tennstedt, PhD

New England Research Institutes, Watertown, MA, US

LP (Luc) de Witte, PhD

Vilans, centre of expertise on long term care, Hoensbroek/Utrecht, The Netherlands

L (Lucy) Yardley, PhD

University of Southampton, School of Psychology, Southampton, UK 


\section{Dankwoord / Acknowledgement}

Dagelijks kunnen we ervaren dat "de ene mens de andere scherpt" (Spreuken 27:17). Dit scherpen van elkaar maakt het leven boeiend en zorgt voor persoonlijke ontwikkeling. Dat velen mij in mijn onderzoek hebben gescherpt blijkt uit dit dankwoord.

Allereerst de begeleiders van mijn promotieproject: promotor Ruud Kempen, copromotor Jolanda van Haastregt en promotor Jacques van Eijk. De wijze waarop elk van jullie mij begeleidde heb ik als zeer prettig ervaren. Ruud en Jolanda, ik had me geen betere directe begeleiders kunnen wensen. Jullie betrokkenheid, constructieve houding en vertrouwen maakte de samenwerking tot een genoegen. Het feit dat ik jullie altijd kon bereiken voor tussentijds overleg waardeer ik enorm. Ruud, "alles begint met een A4-tje" en zo ook je advertentie voor een postdoc en aio die "als tandem aan de slag" moesten. Bedankt dat je mij dit prachtige project toevertrouwde en mij gaandeweg de vrijheid en kansen bood om extra artikelen te schrijven en in andere 'keukens' te kijken. Gescherpt voel ik mij door je wetenschappelijke deskundigheid en je persoonlijke gedrevenheid, nauwkeurigheid en integriteit. Jolanda, wij gingen "als tandem aan de slag" en dat liep gesmeerd! Bedankt voor de prettige samenwerking waarin ruimte was voor het scherpen van gedachten in discussies, maar ook voor gezelligheid en persoonlijke belevenissen. Het enthousiasme en uithoudingsvermogen waarmee jij tijdens de opstartfase aan het cursusprotocol werkte, bewonder ik nu nog. In de afgelopen jaren heb ik veel van je (pragmatische) aanpak en heldere commentaar geleerd. Bedankt voor de ruimte die je mij gaf om het proefschrift met deze artikelen te vullen. Jacques, al in een vroeg stadium stond voor jou vast dat dit promotieproject tot een goede afronding zou komen. Bedankt voor je vertrouwen in mij en voor je bijdragen aan het scherpen van mij. Dankzij jouw suggestie ligt er nu ook een artikel over de ontwikkeling van de Nederlandse interventie. Het enthousiasme waarmee jij op mijn stukken reageerde en je adviezen in de eindfase van het promotieonderzoek heb ik zeer op prijs gesteld.

De leden van de projectgroep en/of coauteurs van de artikelen wil ik hartelijk danken voor hun bijdrage: Erik van Rossum, Sharon Tennstedt, Lucy Yardley, Luc de Witte, Paul Stalenhoef en Ton Ambergen. Erik, je oprechte betrokkenheid bij het project en je belangstelling voor de voortgang werkte motiverend. Met een realistische kijk op zaken en constructief commentaar heb je het project en de artikelen gescherpt. Dank je wel! Sharon, thank you for your sincere and enthusiastic commitment to this project. You shared valuable experiences that facilitated the kick-off of 'Beter in Balans' in The Netherlands. I am also grateful for your clear and useful contributions to the development and effect paper. Lucy, your comments have contributed greatly to the quality and clarity of the systematic review; thank you for participating in this study. Luc, Paul en Ton, bedankt voor jullie bijdragen aan de artikelen en de statistische bewerkingen.

De bijdrage van twee groepen mensen is cruciaal geweest voor de goede afloop van dit project: de deelnemers en de cursusleiders. Allereerst wil ik mijn waardering uitspreken voor de deelnemers in Kerkrade, Heerlen en Maastricht. Bedankt voor de medewerking aan de dataverzameling en de cursus 'Beter in Balans'. Ik heb erg genoten van de gesprekken en cursusbijeenkomsten. Ik hoop van harte dat het onderzoek ook voor u van betekenis is geweest. Mijn waardering gaat ook uit naar 
de wijkverpleegkundigen, die de cursusbijeenkomsten hebben geleid: Til Bemelen, José Grouls, Patrick Haagen, Margo van Haeringen, Judith Janssen en Luc Schurer. De samenwerking met jullie heb ik als zeer prettig ervaren. Jullie positieve en flexibele inzet maakte dat deze praktische kant van het onderzoek als een trein liep. Met jullie enthousiasme voor de cursus enerzijds en kritische kanttekeningen anderzijds was het uitvoeren van de eerste effectanalyses echt spannend. Christien Kooijman van ZorggroepThuis en Pierre Dols van GroenekruisDomicura, bedankt voor de medewerking van de thuiszorgorganisaties aan dit project. Christien, ook bedankt voor je andere projectgerelateerde bijdragen.

Achter alle bovengenoemde namen schuilt nog een reeks mensen die hebben bijdragen aan het project. Een aantal daarvan wil ik noemen. Vonca Schaffers en Laury Quaedackers, samen hebben we duizenden telefoontjes gepleegd, enveloppen gevuld en vragenlijsten ingevoerd. Bedankt voor jullie assistentie! Het MEMIC, in het bijzonder Marlène Ronner, de enquêtrices en Annemie Mordant, bedankt voor jullie inzet en de prettige samenwerking. Ingrid Weijnen, Willem Strik en Evelien Pijpers, bedankt voor jullie betrokkenheid bij het trainen van de wijkverpleegkundigen en/of het ontwikkelen van het cursusprotocol. Tot slot, de Gemeente Heerlen en de Gemeente Maastricht, hartelijk dank voor het beschikbaar stellen van gegevens voor het benaderen van potentiële deelnemers aan het project.

ZonMw, the School for Public Health and Primary Care (Caphri) en het Universiteitsfonds Limburg/SWOL wil ik bedanken voor de financiële bijdragen die het bezoeken van gerontologie congressen mogelijk maakten. The Prevention of Falls Network Europe (EC contract QLK6-CT-2002-02705) is gratefully acknowledged for welcoming me to several interesting meetings.

Diverse (oud)collega's wil ik hier ook noemen. Allereerst twee oud-collega's uit het noorden. Marieke van Heuvelen, bedankt voor je 'duwtje' in zuidelijke richting; de 'matching' was, wat mij betreft, perfect. Lidwien Schure, met je heldere kijk op zaken en juiste verwoording scherp je onze gesprekken die ik erg waardeer. Mijn (oud)collega's bij Medische Sociologie wil ik bedanken voor de gezelligheid, maar ook voor het feit dat voor een praatje of deskundig advies altijd wel een deur openstond. Junioren, ook bedankt voor leuke avondjes die in het teken stonden van promotieonderzoek of gezellig samenzijn. Marjoke, Sylvia, Liliane en Marja, bedankt voor de 'klusjes' die jullie mij uit handen namen en Arnold, bedankt voor de ICT ondersteuning! Jolanda en Annemarie, fijnere kamergenootjes had ik me niet kunnen wensen! Mijn gloednieuwe collega's wil ik bedanken voor de prettige ontvangst in de vakgroep; binnenkort hoop ik letterlijk mijn plekje bij jullie te vinden.

Beste vrienden, bedankt voor alle gezellige koffiepauzes, etentjes, bezoekjes of uitjes in het noorden, midden of zuiden van het land. Ik koester jullie vriendschap en waardeer jullie belangstelling voor mijn werkzaamheden. Bedankt ook voor jullie betrokkenheid bij het afronden van mijn proefschrift, variërend van een belangstellend gesprek tot het geven van 'feedback' op tekst. Annemarie en Else, bedankt voor de wijze waarop jullie vriendschap invulling geven. Ik ben zeer blij dat jullie op 28 februari mijn paranimfen zijn!

Mijn familie wil ik bedanken voor wie ze zijn. Mooie herinneringen koester ik aan mijn lieve pakes en buitengewoon lieve beppes. Op eigen, creatieve wijze zijn zij, ook 
op latere leeftijd, met uitdagingen in het leven omgegaan. Dit zal ik altijd bewonderen. Jan-Menno, Bert en Bernard, als grote broers waren jullie al vroeg mijn voorbeelden en jullie enthousiasme voor allerlei activiteiten werkt nog steeds aanstekelijk. Van onze verschillende karakters en interesses kan ik (stiekem) erg genieten. Bedankt dat jullie er voor mij zijn. Diana en Helma, met jullie heb ik het maar bijzonder goed getroffen! Lisette, Anouk, Afke, Rieneke en Feie, een glimlach van jullie en mijn dag is goed.

Lieve pa en ma, jullie wil ik in het bijzonder bedanken. Jullie vormen voor mij een natuurlijke thuisbasis, met een juiste mate van zorg, vertrouwen en betrokkenheid, die bijdraagt aan wie ik ben. Jullie liefde voor flora, fauna en wetenschap en voor goede (verpleegkundige) zorg voor jong en oud is overduidelijk. Het valt niet te ontkennen dat ik iets van jullie allebei in me heb! 


\section{Publications}

Zijlstra GA, van Haastregt JC, van Eijk JT, de Witte LP, Kempen GI. Mediating effects of psychosocial factors on fear of falling and daily activity in a cognitive behavioral intervention. Submitted.

Zijlstra GA, van Haastregt JC, Ambergen T, van Rossum E, van Eijk JT, Kempen Gl. Effectiveness of a cognitive behavioural group intervention on fear of falling and associated avoidance of activity in community-living older people: a randomised controlled trial. Submitted.

Van Haastregt JC, Zijlstra GA, van Rossum E, van Eijk JTh, Kempen GI. Feelings of anxiety and symptoms of depression in community-living older persons who avoid activity due to fear of falling. Am J Geriatr Psychiatry 2007; in press.

Kempen GI, Yardley L, van Haastregt JC, Zijlstra GA, Beyer N, Hauer K, Todd C. The Short FES-I: a shortened version of the Falls Efficacy Scale-International to assess fear of falling. Age Ageing 2007; doi:10.1093/ageing/afm157.

Kempen GI, Zijlstra GA, van Haastregt JC. Het meten van angst om te vallen met de Falls Efficacy Scale-International (FES-I). Achtergrond en psychometrische kenmerken. Tijdschr Gerontol Geriatr 2007;38(4):204-12.

Van Haastregt JC, Zijlstra GA, van Rossum E, van Eijk JT, de Witte LP, Kempen GI. Feasibility of a cognitive behavioural group intervention to reduce fear of falling and associated avoidance of activity in elderly people living in the community: a process evaluation. BMC Health Serv Res 2007;7:156.

Zijlstra GA, van Haastregt JC, van Eijk JTh, van Rossum E, Stalenhoef PA, Kempen GI. Prevalence and correlates of fear of falling, and associated avoidance of activity in the general population of community-living older people. Age Ageing 2007;36:304-9.

Zijlstra GA, van Haastregt JC, van Rossum E, van Eijk JT, Yardley L, Kempen GI. Interventions to reduce fear of falling in community-living older people: a systematic review. J Am Geriatr Soc 2007;55:603-15.

Kempen Gl, Todd CJ, van Haastregt JC, Zijlstra GA, Beyer N, Freiberger E, Hauer K, PiotZiegler C, Yardley L. Cross-cultural validation of the Falls Efficacy Scale International (FES-I) in older people: results from Germany, The Netherlands and the UK were satisfactory. Disabil Rehabil 2007;29(2):155-62.

Zijlstra GA, Tennstedt SL, van Haastregt JC, van Eijk JT, Kempen GI. Reducing fear of falling and avoidance of activity in elderly persons: The development of a Dutch version of an American intervention. Patient Educ Couns 2006;62(2):220-7. 
Zijlstra GA, van Haastregt JC, van Eijk JT, Kempen Gl. Evaluating an intervention to reduce fear of falling and associated activity restriction in elderly persons: design of a randomised controlled trial [ISRCTN43792817]. BMC Public Health 2005;5(1):26.

Van Heuvelen MJ, Hochstenbach JB, Brouwer WH, de Greef MH, Zijlstra GA, van Jaarsveld E, Kempen GI, van Sonderen E, Ormel J, Mulder T. Differences between participants and non-participants in an RCT on physical activity and psychological interventions for older persons. Aging Clin Exp Res 2005;17(3):236-45. 


\section{About the author}

Rixt Zijlstra was born on December 2, 1973 in the Zuidelijke IJsselmeerpolders, The Netherlands. After completing her secondary education at the Christelijk College Nassau-Veluwe and the Randmeer College in Harderwijk, she participated in a cultural exchange programme in 1994 for which she stayed in New Jersey in the United States for over a year.

In August 1995 she started to study Human Movement Sciences at the University of Groningen. Here she developed her research interest in improving physical and psychosocial functioning in frail and older populations. After an additional internship at the MD Anderson Cancer Center in Houston, Texas in the United States, she graduated with a major in Sports and Physical Activity in August 2000. After her graduation she held two part-time research positions at the University of Groningen. Firstly, she collaborated in the Groningen Intervention Study on Successful Aging (GISSA) at the Center for Human Movement Sciences. GISSA comprised a longitudinal randomised trial evaluating the effects of physical activity and/or psychological training on physical and psychological fitness, and disability in community-living older people. Secondly, she collaborated in Pallas, a study on the needs of family caregivers of people with cancer in the palliative phase, at the Faculty of Medical Sciences.

In March 2002 the author started her PhD-project on fear of falling and avoidance of activity in community-living older people at the Maastricht University, Faculty of Health, Medicine and Life Sciences and School for Public Health and Primary Care (Caphri). This project included a longitudinal randomised trial evaluating the effects of a cognitive behavioural group intervention on fear of falling and associated avoidance of activity in community-living older people. All studies described in this thesis are conducted as part of this PhD-project. Since April 2007 she is affiliated with the same faculty and school as a postdoctoral research fellow. As such, she performs a trial on the impact of orientation and mobility training on participation, mobility and quality of life in older adults with visual impairments. In the near future she will collaborate in the evaluation of an in-home version of the intervention described in this thesis, which will focus explicitly on frail older people. Her current research activities are part of the CAPHRI research program 'Innovations in the care for the elderly'. 


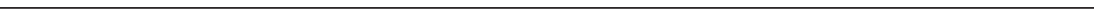

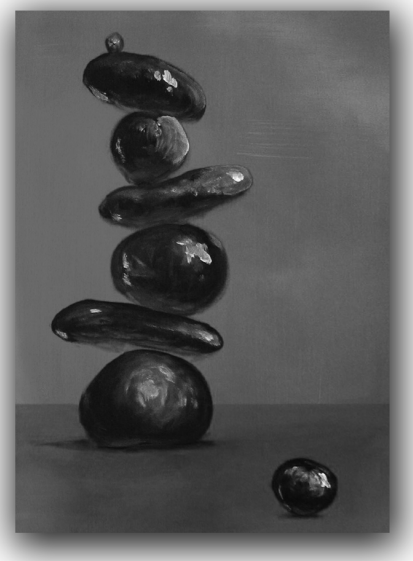

192 